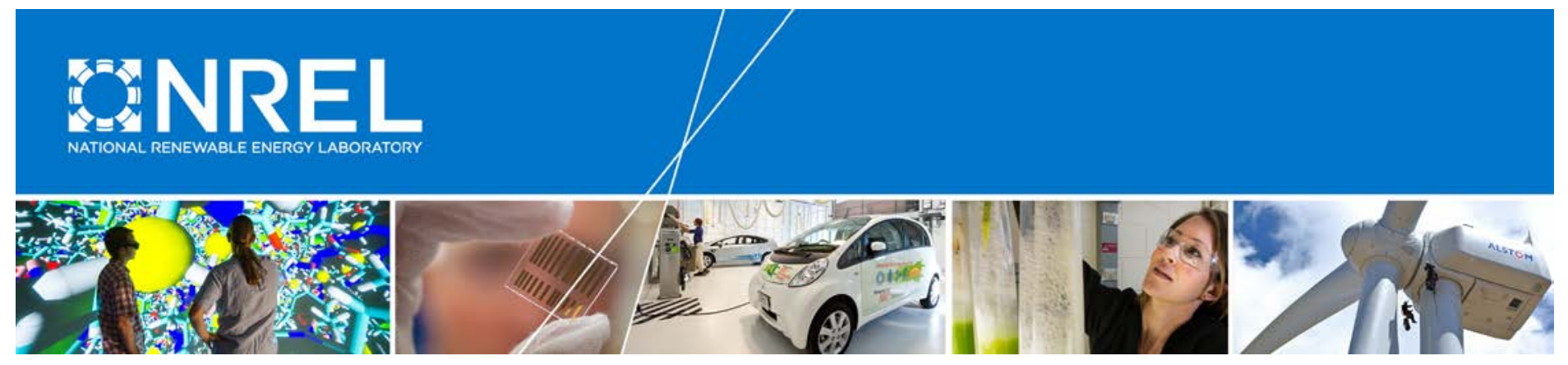

\title{
Western Wind and Solar Integration Study Phase 3A: Low Levels of Synchronous Generation
}

Nicholas W. Miller, Bruno Leonardi, and Robert D'Aquila GE Energy Management

Kara Clark

National Renewable Energy Laboratory

NREL is a national laboratory of the U.S. Department of Energy Office of Energy Efficiency \& Renewable Energy Operated by the Alliance for Sustainable Energy, LLC

This report is available at no cost from the National Renewable Energy Laboratory (NREL) at www.nrel.gov/publications.

Technical Report

NREL/TP-5D00-64822

November 2015 


\section{Western Wind and Solar Integration Study Phase 3A: Low Levels of Synchronous Generation}

Nicholas W. Miller, Bruno Leonardi, and

Robert D'Aquila

GE Energy Management

Kara Clark

National Renewable Energy Laboratory

Prepared under Task No. WE14.9B04

NREL is a national laboratory of the U.S. Department of Energy Office of Energy Efficiency \& Renewable Energy Operated by the Alliance for Sustainable Energy, LLC

This report is available at no cost from the National Renewable Energy Laboratory (NREL) at www.nrel.gov/publications.

National Renewable Energy Laboratory 15013 Denver West Parkway Golden, CO 80401

303-275-3000 • www.nrel.gov

\section{Technical Report}

NREL/TP-5D00-64822

November 2015

Contract No. DE-AC36-08GO28308 


\title{
NOTICE
}

This report was prepared as an account of work sponsored by an agency of the United States government. Neither the United States government nor any agency thereof, nor any of their employees, makes any warranty, express or implied, or assumes any legal liability or responsibility for the accuracy, completeness, or usefulness of any information, apparatus, product, or process disclosed, or represents that its use would not infringe privately owned rights. Reference herein to any specific commercial product, process, or service by trade name, trademark, manufacturer, or otherwise does not necessarily constitute or imply its endorsement, recommendation, or favoring by the United States government or any agency thereof. The views and opinions of authors expressed herein do not necessarily state or reflect those of the United States government or any agency thereof.

This report is available at no cost from the National Renewable Energy Laboratory (NREL) at www.nrel.gov/publications.

Available electronically at SciTech Connect http:/www.osti.gov/scitech

Available for a processing fee to U.S. Department of Energy and its contractors, in paper, from:

\author{
U.S. Department of Energy \\ Office of Scientific and Technical Information \\ P.O. Box 62 \\ Oak Ridge, TN 37831-0062 \\ OSTI http://www.osti.gov \\ Phone: 865.576.8401 \\ Fax: 865.576.5728 \\ Email: reports@osti.gov
}

Available for sale to the public, in paper, from:

\author{
U.S. Department of Commerce \\ National Technical Information Service \\ 5301 Shawnee Road \\ Alexandria, VA 22312 \\ NTIS http://www.ntis.gov \\ Phone: 800.553 .6847 or 703.605 .6000 \\ Fax: 703.605.6900 \\ Email: orders@ntis.gov
}




\section{Acknowledgments}

The National Renewable Energy Laboratory (NREL) and General Electric (GE) gratefully acknowledge the support of Charlton Clark from the U.S. Department of Energy, Office of Energy Efficiency and Renewable Energy, Wind and Water Power Technologies Office for funding this work.

NREL and GE also thank the members of the technical review committee for their insightful comments and assistance. Participation in the committee does not imply agreement with the project findings. The committee included:

- Mark Ahlstrom, WindLogics

- William Anderson, Xcel Energy

- Jamie Austin, PacifiCorp

- Ron Belval, Tucson Electric Power

- Ray Byrne, Sandia National Laboratories

- Thomas Carr, Western Governors' Association

- Malati Chaudhary, Public Service Company of New Mexico

- Charlton Clark, U.S. Department of Energy

- Bob Cummings, North American Electric Reliability Corporation

- Donald Davies, Western Electricity Coordinating Council

- Tom Duane, Public Service Company of New Mexico

- Bob Easton, Western Area Power Administration

- Sara Eftekharnejad, Tucson Electric Power

- Erik Ela, Electric Power Research Institute

- Ryan Elliott, Sandia National Laboratories

- Abe Ellis, Sandia National Laboratories

- Irina Green, California Independent System Operator

- Doug Larson, Western Governors' Association

- Eddy Lim, Federal Energy Regulatory Commission

- Clyde Loutan, California Independent System Operator

- Jim McCalley, Iowa State University

- Mark O’Malley, University College Dublin

- Brian Parsons, Western Grid Group

- Cesar Silva-Monroy, Sandia National Laboratories 
- Vikas Singhvi, Electric Power Research Institute

- Charlie Smith, Utility Variable-Generation Integration Group

- Brian Stringer, Western Area Power Administration

- Vijay Vittal, Arizona State University

- Janice Zewe, Sacramento Municipal Utility District

NREL regrets any inadvertent omission of any project participants and contributors. 


\section{List of Acronyms}

CSCR
CSP
DG
ERMVA
GE
GW
HVDC
Hz
LCC
LVPL
mHz
min
MVA
MW
NERC
NREL
OEM
PV
RAS
REMTF
S
SCMVA
SCR
VSC
WECC
WTG
WWSIS

composite short-circuit ratio

concentrating solar thermal power plant

distributed generation, embedded PV

effective renewable MVA

General Electric

gigawatt

high-voltage direct current

Hertz

line commutated converter

low-voltage power limit

millihertz

minute

megavolt ampere

megawatt

North American Electric Reliability Corporation

National Renewable Energy Laboratory

original equipment manufacturer

photovoltaic solar power, utility-scale photovoltaic

power plant

remedial action scheme

WECC Renewable Energy Modeling Task Force

second

short-circuit MVA

short-circuit ratio

voltage source converter

Western Electricity Coordinating Council

wind turbine generator

Western Wind and Solar Integration Study 


\section{Executive Summary}

The stability of the North American electric power grids under conditions of high penetrations of wind and solar is a significant concern and possible impediment to reaching renewable energy goals. The $33 \%$ wind and solar annual energy penetration considered in this study results in substantial changes to the characteristics of the bulk power system. This includes different power flow patterns, different commitment and dispatch of existing synchronous generation, and different dynamic behavior from wind and solar generation.

The Western Wind and Solar Integration Study (WWSIS), sponsored by the U.S. Department of Energy, is one of the largest regional solar and wind integration studies to date. In multiple phases, it has explored different aspects of the question: Can we integrate large amounts of wind and solar energy into the electric power system of the West? The work reported here focused on the impact of low levels of synchronous generation on the transient stability performance in one part of the region in which wind generation has displaced synchronous thermal generation under highly stressed, weak system conditions. It is essentially an extension of WWSIS-3.

Transient stability, the ability of the power system to maintain synchronism among all elements following disturbances, is a major constraint on operations in many grids, including the western U.S. and Texas systems. These constraints primarily concern the performance of the large-scale bulk power system. But grid-wide stability concerns with high penetrations of wind and solar are still not thoroughly understood. This work focuses on "traditional" fundamental frequency stability issues, such as maintaining synchronism, frequency, and voltage.

The objectives of this study are to better understand the implications of low levels of synchronous generation and a weak grid on overall system performance by:

- Investigating the Western Interconnection under conditions of both high renewable generation (e.g., wind and solar) and low synchronous generation (e.g., significant coal power plant decommitment or retirement)

- Analyzing both the large-scale stability of the Western Interconnection and regional stability issues driven by more geographically dispersed renewable generation interacting with a transmission grid that evolved with large, central station plants at key nodes.

Leading into this study, WWSIS-3 delved into the dynamic performance of the grid in the fractions of a second to 1 min following a large disturbance (e.g., loss of a large power plant or a major transmission line), which is critical to system reliability. In particular, that study examined the large-scale transient stability and frequency response of the Western Interconnection with high penetrations of wind and solar, and it identified means to mitigate any adverse performance impacts via transmission reinforcements, storage, advanced control capabilities, or other alternatives (Miller et al. 2014a, Miller et al. 2014b). Key findings included:

- With good system planning, sound engineering practices, and commercially available technologies, the Western Interconnection can withstand the crucial first minute after grid disturbances with high penetrations of wind and solar.

- Local stability, voltage, and thermal problems can be addressed with traditional transmission system reinforcements (e.g., transformers, shunt capacitors, local lines). 
- Nontraditional frequency-responsive controls on wind, utility-scale solar photovoltaic power (PV), concentrating solar thermal power (CSP), and energy storage are effective at improving frequency response after the loss of approximately 2,750 MW of central station generation.

- Load-modeling assumptions can have as much impact on system performance as high penetrations of wind and solar. Accurate modeling of load, as well as renewable generation, is extremely important when analyzing high-stress conditions.

As noted above, the work reported here is an extension of the research performed in WWSIS-3.

\section{Transient Stability Discussion}

In addition to maintaining the balance between electricity generation and electricity demand, power system operators must ensure that the grid can successfully transition from normal operation (e.g., all transmission lines and generating units are in service), through a disturbance (e.g., an abrupt outage of a major transmission line or large generator), and into a new stable operating condition in the 10-20 s immediately following a disturbance. The ability to make this successful transition is called transient stability.

Figure ES-1 shows an updated visualization of the problem originally presented in Vittal (2003). The round masses represent synchronous generators, and the various springy lines connecting the masses represent transmission lines. The scissors represent a disturbance, which might cut a line or disconnect a generator. The rubbery mass-spring system bounces around. If the event is too severe or some of the lines are stretched too tight (i.e., have too much loading), more lines will break. It is easy to imagine a cascading failure in which each successive break leads to another. A substantial part of system planning is aimed at avoiding such unacceptable consequences.

To further elaborate on the example, the hands represent the controllable nature of inverter-based generation, either wind or PV. These controls can be tuned to provide specific types of responses to disturbances, and this is explored in this report.

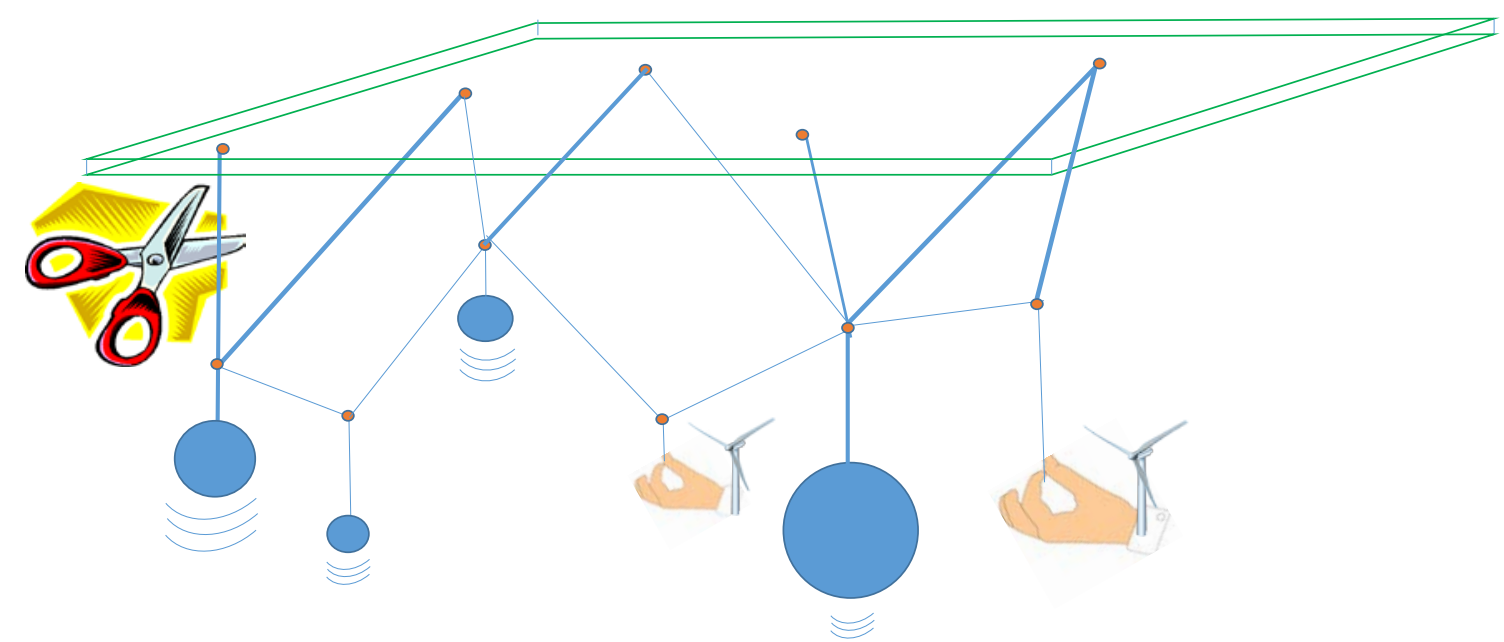

Figure ES-1. Visualization of transient stability with synchronous generators and wind power plants 
The Western Interconnection has a long history of constraints because of transient stability limitations that vary depending on system characteristics such as the level of electricity demand (e.g., peak summer load), the amount of power flowing on the transmission system (e.g., heavy flows on critical paths), and the location of the generating plants in operation (e.g., remote from population centers). The primary criteria of acceptable transient stability are according to reliability standards set by the North American Electric Reliability Corporation and the Western Electricity Coordinating Council (WECC). Transient stability can be both systemic and local. Large penetrations of inverter-based, or nonsynchronous, wind and solar generation may substantially alter system stability as a result of changes in angle/speed swing behavior caused by reduced inertia or changes in voltage swing behavior because of different voltage control systems, different power flow patterns, and the displacement of synchronous generation at key locations.

\section{Weak Grid Description}

One of the most challenging operating conditions for inverter-based generation is in a so-called "weak grid." But what is a weak grid? Analytical descriptions have long been available, but in essence, the question is one of relative size: Is the amount of inverter-based generation relatively small or relatively large compared to the amount of synchronous generation connected to the host grid? Historically, wind power plants were relatively small compared to the grid. A 1-MW wind turbine connected to a $20-\mathrm{GW}$ system has no more ability to move that system than a lap dog, even a badly behaved one, can bend a palm tree (Figure ES-2). However, as wind power plants become larger and are connected into smaller and more remote portions of the grid, they exert more influence. A 200-MW wind power plant connected to a 300-MW subsystem can now impact system response to a disturbance similar to the way in which an elephant can bend or break a palm tree unless it is trained not to (Figure ES-3). It is this latter condition of high penetrations of wind and low levels of synchronous generation in a relatively small and remote part of the Western Interconnection that is the focus of this report.

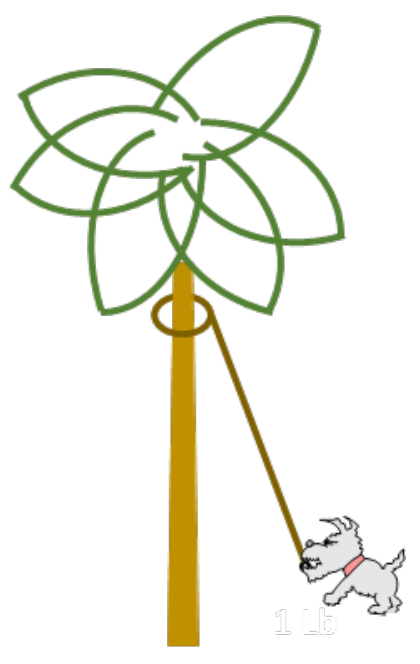

Figure ES-2. Small inverter-based power plant relative to grid

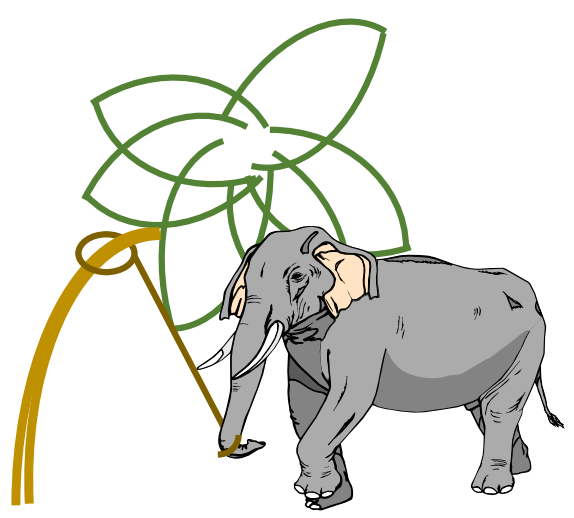

Figure ES-3. Large inverter-based power plant relative to grid 


\section{Study Model and Assumptions}

The selection of the initial condition for the stability analysis was a key consideration. In the WWSIS-3 study process, lengthy discussions were held with the study's technical review committee regarding which conditions should be examined. Some of that decision-making process is recorded in the study report, but it is useful to provide some context here. To evaluate the impact on transient stability and frequency response of high levels of wind and solar generation (or any other type of inverter-based resource-for example, most types of energy storage), it is useful to select conditions in which the penetration levels of these resources are high. Further, it is well known that light load conditions represent some of the more challenging conditions, especially for frequency response, due to the limited amount of frequency-responsive synchronous generation online. Thus, light spring conditions with high levels of wind and solar are of particular interest.

Three scenarios were evaluated. The Light Spring Base case represents a future in which the current renewable portfolio standard targets are met. The Light Spring Hi-Mix case was built form the Base case but includes even higher levels of wind and solar generation. It represents a snapshot in time: a windy, sunny morning in the spring. The details (e.g., renewable generation plant MW output, conventional generation redispatch/decommitment) were mined from WWSIS-2 and are reported in Miller et al. (2014a, 2014b). The Light Spring Extreme case has an even higher penetration of wind and solar generation. Both the Hi-Mix and Extreme cases have the same "high renewable" topology - i.e., the same plants at the same buses with the same ratings. The differences are in the wind and solar power plant outputs and the associated redispatch and decommitment of the rest of the generation fleet.

The U.S. portion of load in WECC in all three cases is $89 \mathrm{GW}$, and the U.S. portion of wind and solar production totals in the three cases is as follows:

- Light Spring Base: Approximately $24 \mathrm{GW}$ wind and solar production

- Light Spring Hi-Mix: Approximately $56 \mathrm{GW}$ wind and solar production

- Light Spring Extreme: Approximately $63 \mathrm{GW}$ wind and solar production.

Figure ES-4 shows the generation penetration duration curves for all of WECC for the original WWSIS-2 production simulation Hi-Mix case (blue), and Base case (red). A duration curve displays data sorted from the highest value to the lowest. Thus, the sequence has no chronological information. The curves show 5-min resolution data for the study year, so there are slightly more than 100,000 samples per year. Each data point is the portion of all generation that is wind plus solar power production during one 5-min period. Instantaneous penetration for the three study cases - Base, Hi-Mix, and Extreme - are pointed out in the figure. The Base and HiMix cases are in the top $10 \%$ of hours for the year; the difference in penetration is due to the difference in installed wind and solar generation. The extreme case is in the top less than $1 / 2$ percent; the difference between the Hi-Mix and Extreme cases is due to higher speed wind and higher intensity solar on the same installed base of equipment.

Although these cases were expected to be both challenging and illuminating for this investigation, there is no implication that these cases are necessarily the most difficult in all 
regards. As stated elsewhere, this investigation is not a substitute for the complete, detailed planning analysis that must accompany a massive evolution of a system.

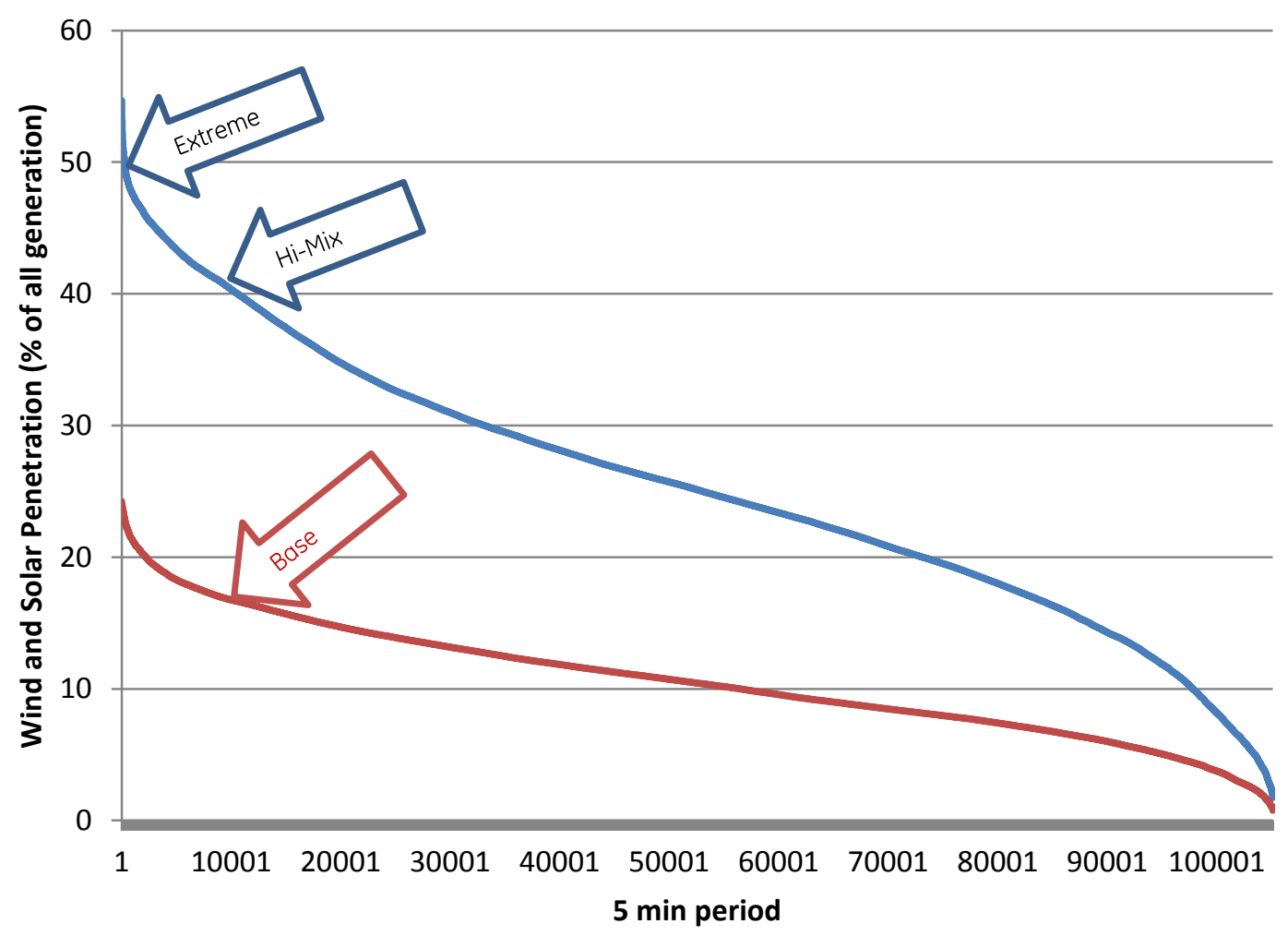

Figure ES-4. Penetration duration curve from WWSIS-2 production simulations

\section{Database Refinement}

The database for this study was refined to bring the Light Spring cases from WWSIS-3 into closer alignment with today's consensus of what the system might look like in 2022. Inputs from the technical review committee were collected to determine any necessary changes to the generation mix or transmission topology.

In addition, many proposed CSP plants in the WWSIS-3 databases were converted to utilityscale PV projects, because the market for CSP in the western United States largely disappeared after those databases were developed. For this study, all existing and high-probability new CSP plants (i.e., plants under construction or fully financed) were retained. Thus, the Hi-Mix and Extreme cases include 3.3 GW of CSP. All other CSP plants (approximately $5.1 \mathrm{GW}$ ) from the WWSIS-3 databases were converted to utility-scale PV.

Summaries of the wind and solar generation in the Light Spring Hi-Mix and Extreme cases are shown in Figure ES-5 and Table ES-1, and Figure ES-6 and Table ES-2, respectively. Totals and penetration figures are for the U.S. portion of WECC only. 


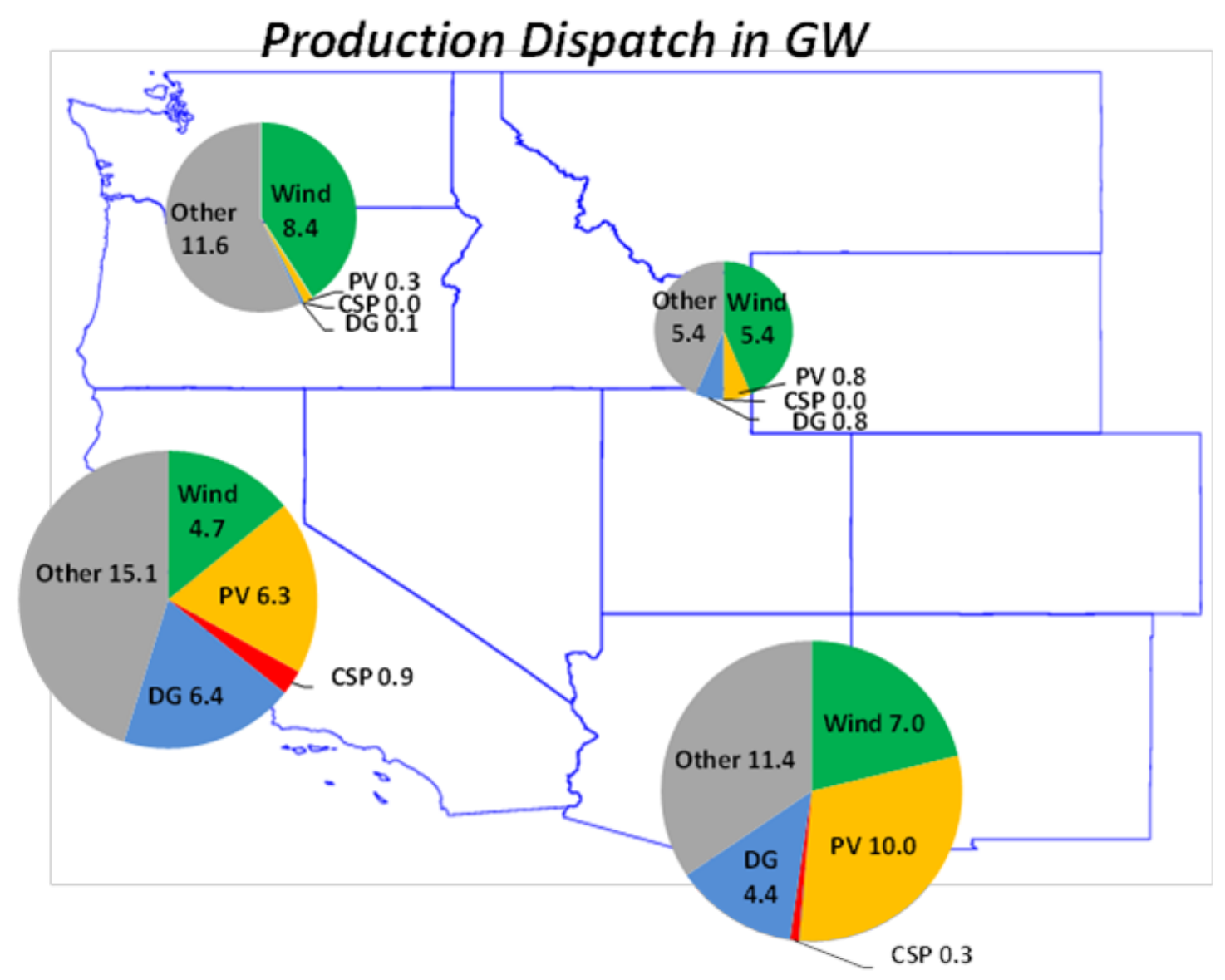

Figure ES-5. Wind and solar generation in the Light Spring Hi-Mix case

Table ES-1. Wind and Solar Generation in the Light Spring Hi-Mix Case

\begin{tabular}{llllll}
\hline & $\begin{array}{l}\text { WECC } \\
\text { (U.S.A.) }\end{array}$ & California & DSW & Northeast & Northwest \\
\hline Wind (GW) & 25.5 & 4.7 & 7.0 & 5.4 & 8.4 \\
PV (GW) & 17.4 & 6.3 & 10.0 & 0.8 & 0.3 \\
CSP (GW) & 1.2 & 0.9 & 0.3 & 0.0 & 0.0 \\
DG $^{a}(\mathrm{GW})$ & 11.7 & 6.4 & 4.4 & 0.8 & 0.1 \\
Others (GW) & 43.6 & 15.1 & 11.4 & 5.4 & 11.6 \\
\hline Total (GW) & 99.5 & 33.5 & 33.1 & 12.4 & 20.4 \\
\hline Penetration & $56.2 \%$ & $54.8 \%$ & $65.6 \%$ & $56.6 \%$ & $43.0 \%$ \\
(\% of U.S. generation dispatch) & & & & & \\
\hline
\end{tabular}

${ }^{\mathrm{a}}$ Distributed generation

${ }^{1}$ The penetration levels shown in this table were calculated considering only the U.S. portion of WECC generation. 


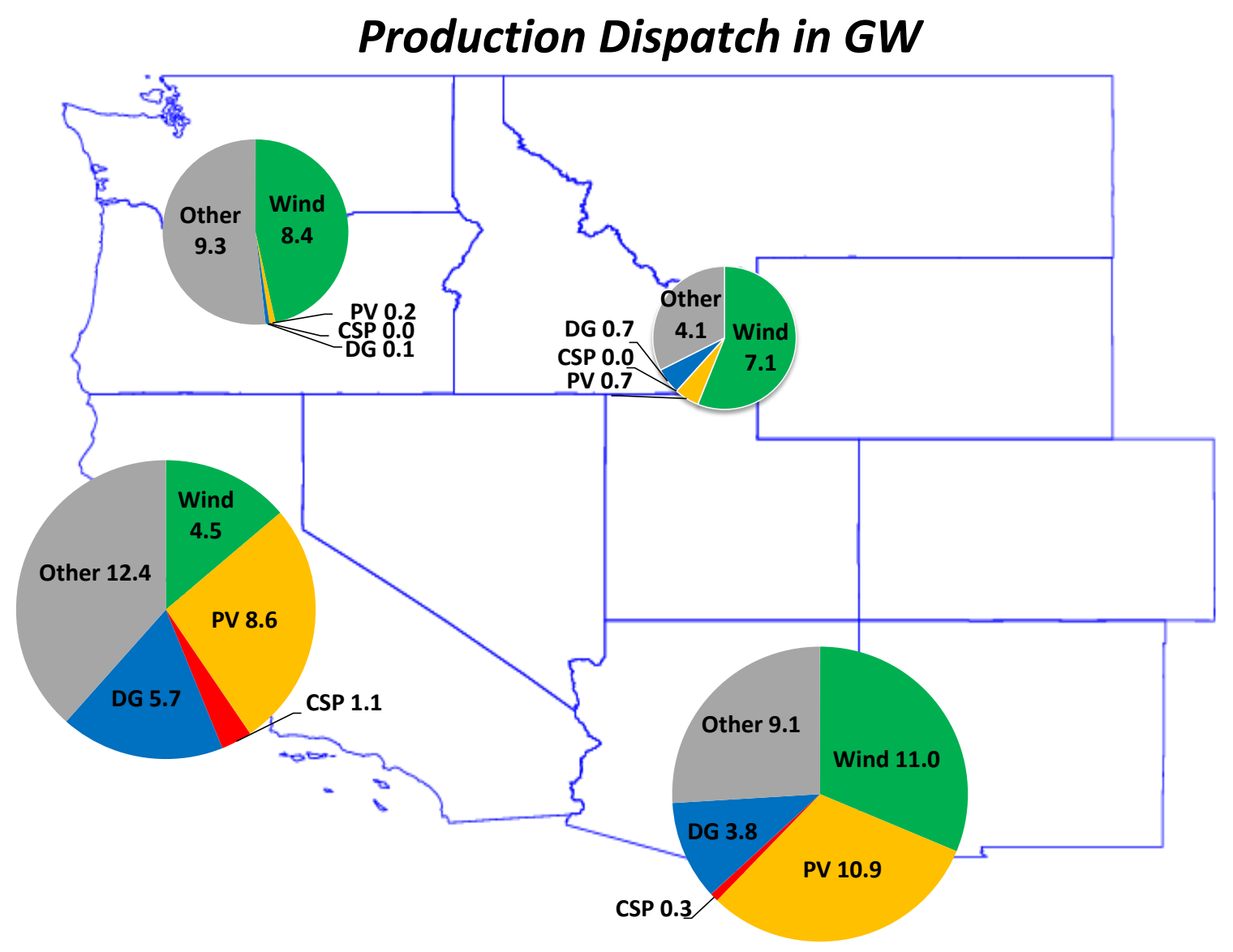

Figure ES-6. Wind and solar generation in the Light Spring Extreme case

Table ES-2. Wind and Solar Generation in the Light Spring Extreme Case

\begin{tabular}{llllll}
\hline & WECC (U.S.A.) & California & DSW & Northeast & Northwest \\
\hline Wind & 30.9 & 4.5 & 11.0 & 7.1 & 8.4 \\
PV & 20.4 & 8.6 & 10.9 & 0.7 & 0.2 \\
CSP & 1.4 & 1.1 & 0.3 & 0.0 & 0.0 \\
DG & 10.4 & 5.7 & 3.8 & 0.7 & 0.1 \\
Others & 34.9 & 12.4 & 9.1 & 4.1 & 9.3 \\
\hline Total (GW) & 98.1 & 32.3 & 35.2 & 12.6 & 18.0 \\
\hline $\begin{array}{l}\text { Penetration } \\
\text { (\% of U.S. }\end{array}$ & $64.4 \%$ & $61.6 \%$ & $74.0 \%$ & $67.6 \%$ & $48.3 \%$ \\
generation & & & & \\
dispatch) & & & & & \\
\hline
\end{tabular}

\footnotetext{
${ }^{2}$ The penetration levels shown in this table were calculated considering only the U.S. portion of WECC generation.
} 


\section{Transient Stability Analysis}

To examine the transient stability of the system with low levels of synchronous generation, a sequence of tests was performed for a severe fault in Wyoming. The future Aeolus $500-\mathrm{kV}$ bus is the eastern terminus of the planned 500-kV AC Gateway West and Gateway South projects. These projects will provide, among other benefits, increased ability to export wind power from that wind-rich region. A severe, three-phase fault at the Aeolus $500-\mathrm{kV}$ bus, cleared by primary protection in four cycles with the trip of the Gateway South line, causes a regionally acute voltage depression and subsequently degraded export capability.

The entire region, and especially eastern Wyoming and northeastern Colorado, has very high levels of wind generation in the Hi-Mix and Extreme cases, and most of the coal power plants in the region are not running. The three combined challenges - severe fault, high wind power, and greatly reduced synchronous generation - are ideal for the intended focus of this study: transient stability with low levels of synchronous generation.

The system was found to have satisfactory transient stability behavior for both the Base case and the Hi-Mix case, but, as was observed in WWSIS-3, the system was unstable for this fault in the Extreme case. Much of the subsequent investigation focused on the causes and possible mitigation of this instability. The key elements of each portion of that investigation are summarized here.

For the initial tests on the Extreme case, a rapid voltage collapse and system separation between the fault in central Wyoming and the eastern extremities of the system were observed. Separation occurred before the fault was cleared. Traditional voltage stability analysis confirmed that the observed behavior is essentially transient voltage collapse and is less closely related to typical machine angular transient stability. This was due to an excessive amount of power flow on two $230-\mathrm{kV}$ lines from a wind power plant that was oversized for the site, creating overloads that were in violation of normal WECC operating rules. Plant output was reduced by approximately $300 \mathrm{MW}$ to bring the loading within steady-state thermal limits. This $300-\mathrm{MW}$ curtailment is from $63 \mathrm{GW}$ of wind and solar generation - or less than $1 / 2$ percent. Tests on that case showed better performance; however, additional mitigation was required to meet reliability criteria. Various wind power plant controls were tested, including those that rapidly reduce power output during voltage depressions and original equipment manufacturer weak grid controls. Acceptable performance could be achieved with combinations of traditional mitigation strategies (e.g., generation reduction, transmission system reinforcement) and nontraditional wind power plant controls.

Tests were also performed to examine the impact of various load models, wind power plant models, and wind power plant control features on system performance. Each aspect was found to have significant impact on results under these extremely high-stress conditions. Tests showed that higher functionality wind power plants tended to reduce the need for other traditional mitigation.

The study also introduced concepts for static screening of the system for weak points that might be particularly vulnerable to transient voltage collapse induced by inverter-based resources. That screening, which used short-circuit strength and a topological estimate of wind power plant 
impact on bus voltages, showed considerable promise by successfully identifying the locations in the system that were proven, by time simulation, to be ill behaved.

\section{Exploration of HVDC Ties into High Wind Region}

The construction of large high-voltage DC (HVDC) ties electrically connecting wind-rich regions to major load centers has long been considered a potential solution to move large amounts of wind energy from remote locations to where it is needed most. In fact, most of the regions that have abundant wind potential in the Western Interconnection are located relatively far away from large consuming centers. Therefore, this study explored how HVDC lines will perform when they serve as major corridors for wind-generated power.

A high-level investigation of the impact of a new, hypothetical, 3,000-MW HVDC link connecting eastern Wyoming, a region that has significant wind potential, and the Las Vegas area, which is predominantly a load center, was performed. The HVDC link was substituted for the planned 500-kV AC Gateway South project, which was rated approximately 2,000 MVA. No changes were made to the generation commitment and dispatch throughout the entire system.

The addition of a large HVDC link introduces important new performance concerns. In particular, if an HVDC link carrying 3,000 MW is suddenly blocked (because of a fault or an unintended trip), system-wide stability implications must be studied and well understood. For that purpose, several scenarios considered blocking the HVDC link.

For the dispatch conditions and commitment tested, the 3,000-MW HVDC link relieved stress more than the 500-kV Gateway South AC project. The DC line carried more than 1,000 MW more than Gateway South, most of which reduced loading on Gateway West. Blocking the link, and dropping 3,000 MW of export, was tolerated easily.

The loss of the Gateway West 500-kV AC line in addition to the loss of the HVDC system can be viewed as an N-1-1 event, critical to system-wide stability. To avoid instability for this postulated event, the system needs both operational actions and physical mitigation. Overall, standard utility practice for mitigation - including redispatch, remedial action schemes, and dynamic and static reactive support - was shown to be effective in this exercise. Additional investigations of even heavier regional exports, with Gateway West carrying higher flows, will help gain more insights on system-wide instability with the loss of the HVDC link.

The high-level investigation reported here suggests that the integration of large blocks of HVDC for export is feasible. Substantial, project-specific engineering far beyond the scope of this investigation would be needed to pick DC technology, rate equipment, and design integration. This investigation provides some observations specific to HVDC for export from regions that have high levels of wind and low levels of synchronous generation:

- A large DC system will have the potential to disrupt the system if not properly integrated.

- Familiar challenges associated with HVDC blocking will need to be carefully considered. Fast voltage support and adapting to rapid counterflows on secondary AC transmission can be challenging. Dynamic reactive compensation is important. 
- The conversion of a coal power plant to a synchronous condenser was found again to be beneficial.

- This investigation did not identify a compelling reason to favor line-commutated converters (LCC) or voltage source converters (VSC) (nor was it intended to), and careful evaluation of specific DC technologies is necessary for each application.

- The potential for local, non-fundamental frequency interaction between inverter-based generation (wind and solar) and large HVDC of any technology must be considered with a predominantly wind-exporting system.

The issues observed in this HVDC investigation are not new to system planners and can be remediated with good planning practices. It is noteworthy to mention that when instability occurs on weak grids that have substantial amounts of renewable generation, the system can become unstable faster than stronger grids do.

\section{Key Observations on Low Levels of Synchronous Generation}

The investigation of transient stability in the eastern portion of the Western Interconnection under conditions of low levels of synchronous generation produced the following insights:

- As with any significant system change, thermal and voltage issues must be addressed before stability issues. Standard techniques can be employed, such as new transmission, reactive compensation, adjusted commitment, and dispatch.

- Transient stability issues in exporting regions when dominated by inverter-based power sources can look different. Behavior is more closely akin to traditional fast voltage collapse than machine-angle stability. Stability failures can occur very rapidly.

The failures observed are moderately localized, because the study area is both highly stressed and on the eastern edge of the Western Interconnection. A key finding is that remediation of performance problems includes two broad classes of technology:

- Standard grid-based fixes, such as reduced loading, transmission reinforcements, remedial action schemes, and reactive compensation with adequate dynamic range.

- Use of control features on wind turbines and wind power plants.

- Use of features in the WECC second-generation, generic models was effective.

○ Individual manufacturer controls can be highly effective.

$\circ$ Wind generation behavior during disturbances varies among manufacturers and features. Attention should be given to the features and performance of specific wind power plants when they are evaluated during the generation interconnection process.

- Grid-based solutions and wind power plant controls for weak grids are complementary. Wind power plants that have higher functionality tend to reduce the need for grid-based solutions and vice versa. 
○ Costs and benefits of the various means of mitigation (e.g., grid-based operational solutions, grid reinforcements, added control features on new wind power plants) should be analyzed to ensure a technically and economically viable solution.

Finally, an analysis of transient stability limitations requires careful attention to models and tools. For instance:

- Simulations with greatly reduced synchronous generation and high stress are strongly affected by details of wind power plant, load, and reactive compensation models.

- After plants are constructed, performance and modeling should be verified for addition to common study data sets.

- Standard transient stability tools using fundamental frequency, positive sequence have built-in approximations that can affect results, particularly when stability limits are hit in very short (e.g., a few cycles) time frames. Enhanced voltage stability analysis tools are needed. Point-on-wave and frequency domain analysis may be required in some circumstances to augment fundamental frequency, positive sequence analysis.

- Other inverter-based generation (e.g., PV) and resources (e.g., battery energy storage) have many similarities in dynamic performance characteristics, and they can be expected to present similar grid behavior and modeling challenges.

\section{Conclusions}

This investigation shows that the integration of large amounts of wind generation in an exporting region with the associated displacement of substantial thermal generation is technically feasible.

It is possible to have transient stability problems, particularly if the system is unduly stressed. The stability problems can look somewhat different. Faults that occur when the transmission system is overloaded in violation of normal operating rules can result in fast voltage collapse and separation.

For the conditions studied - which included highly stressed power flow conditions, high wind and solar generation levels, and the vast majority of major coal generation in the region offline - system stability can be maintained with available wind power plant controls and limited, standard technology grid support.

This is relatively new ground for the industry, and this investigation is not a substitute for detailed planning, but the risks illustrated can be analyzed and mitigated. Tools, data, and the current state-of-the-art interconnection and bulk power system stability studies, if used following good system engineering practice as the system is built out, will ensure continued reliability of the power system.

\section{Future Work}

This work illuminates a number of areas for which further study, analysis using different tools, and the development of new tools would move the industry's understanding of grid operations with low levels of synchronous generation forward. 


\section{Western Interconnection Analysis}

Further investigations of different network topologies with low levels of synchronous generation (e.g., Southern California) would be of particular interest. The concern here is likely to be postfault voltage recovery under peak load, weak grid conditions rather than the dynamic response of renewable generation.

\section{HVDC Options}

Investigations of the relative merits of dedicated compared to embedded HVDC transmission projects for the delivery of wind power to load centers should consider the economics of operations (i.e., include production simulations) and stability evaluation, with particular attention to identifying stability limits such as the impact on relevant path ratings. The investigations could also continue the evaluations of the relative merits of different HVDC technologies (i.e., VSC compared to LCC and point-to-point compared to multi-terminal).

\section{Voltage Stability Tools}

There is a need to develop and adapt voltage stability analysis tools to help planners understand the risks of the type of instability shown in this study. Methods to quickly and meaningfully calculate PV and QV curves that reflect the dynamic constraints of a system that has a preponderance of inverter-based resources would be a valuable complement to traditional transient stability simulations. Similarly, and ideally in coordination, the development of new screening tools based on short-circuit strength and the size, amount, and character of nearby inverter-based generation would be valuable for both planners and developers.

\section{Model and Method Validation}

Analyses of system stability using fundamental frequency, positive sequence analysis are well established and include well-known approximations. This remains the case with high levels of wind and solar. However, the industry's understanding and quantification of the limits of, and the errors introduced by, these modeling assumptions when applied to weak grids that have high levels of inverter-based generation is less well established than for grids dominated by synchronous machines. The use of other tools - such as lab tests on actual equipment, simulations with point-on-wave tools, and field measurements to complement and further validate the models — would strengthen the industry's understanding.

\section{Protection and Relaying Investigation}

Analyses of system stability using fundamental frequency, positive sequence analysis, including those presented in this report, generally assume that protective relaying and protection (e.g., circuit breakers), will continue to perform as presently modeled. For example, the faults used in this study were assumed to be of a "standard" four-cycle duration. The fault-clearing times used in stability studies are usually conservative approximations of actual protection system behavior. This behavior is based on the level and character of short-circuit currents, which are substantially different from and less well known than inverter-based generation. Whether these assumptions are still valid in systems with predominantly inverter-based generation warrants further investigation. 


\section{Contents}

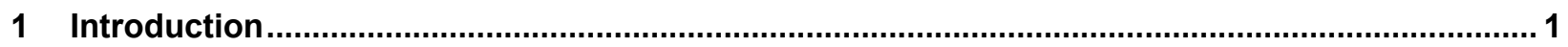

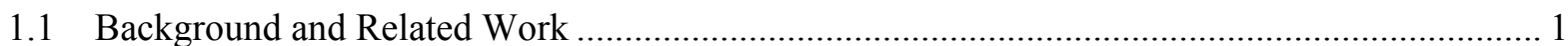

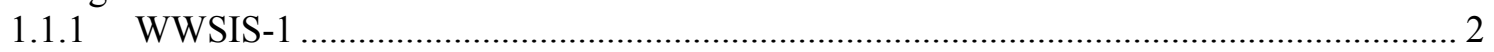

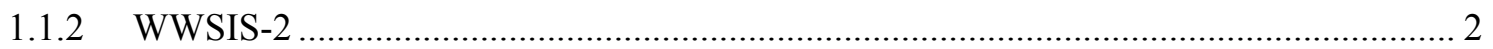

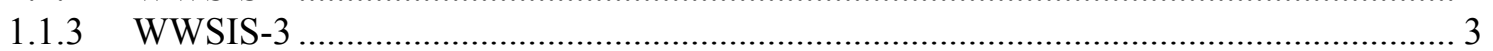

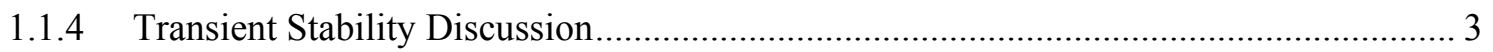

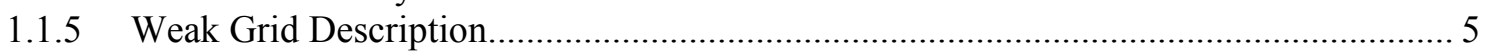

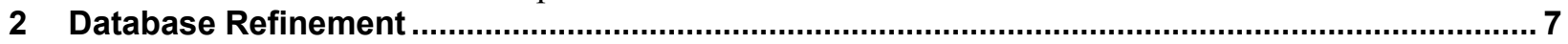

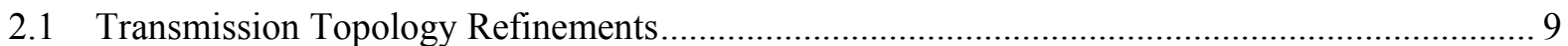

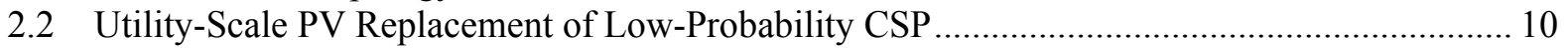

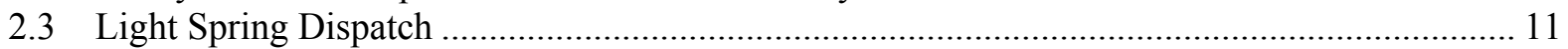

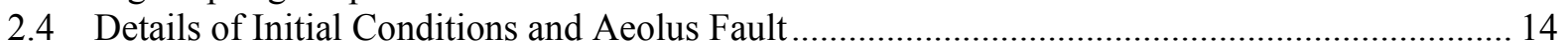

2.5 Model Replacement and Performance Validation........................................................ 16

3 System Transient Stability Performance ......................................................................... 17

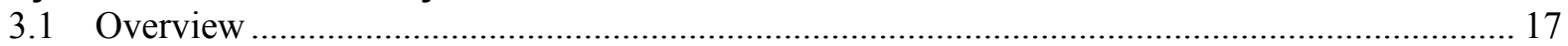

3.2 Initial Performance with Aeolus Fault ............................................................... 17

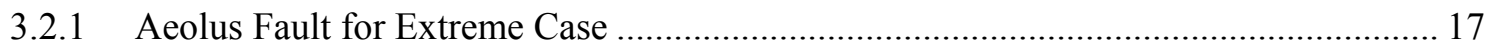

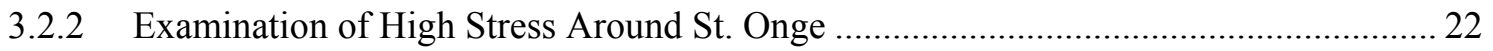

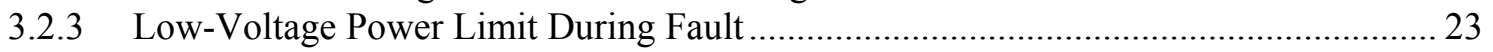

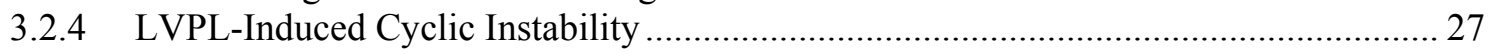

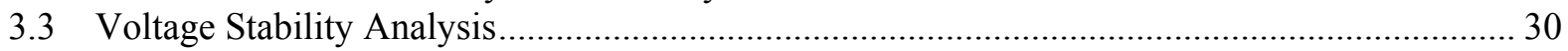

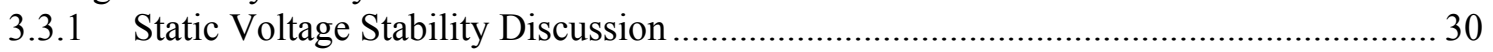

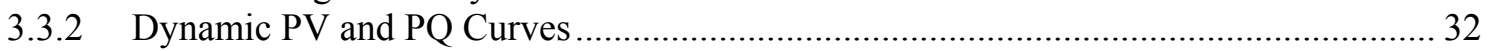

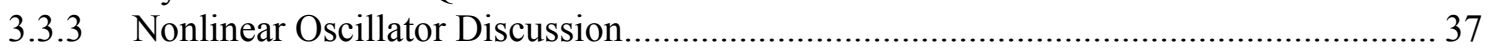

3.4 Observations on Aeolus Fault for Extreme Cases ................................................................. 37

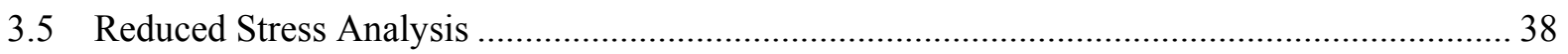

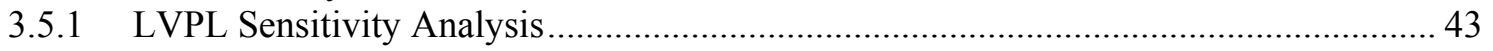

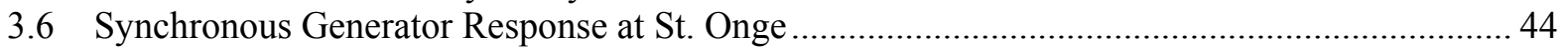

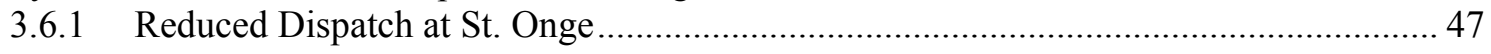

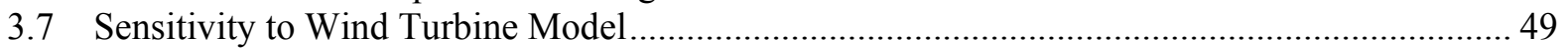

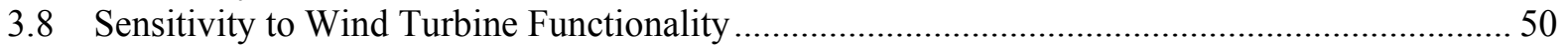

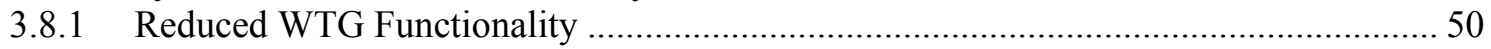

3.8.2 A Manufacturer-Specific WTG Model .................................................................. 51

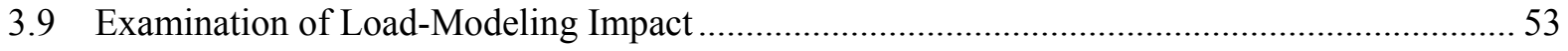

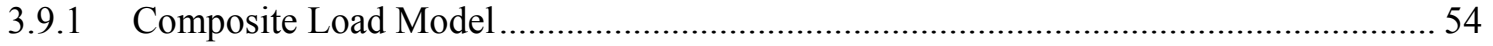

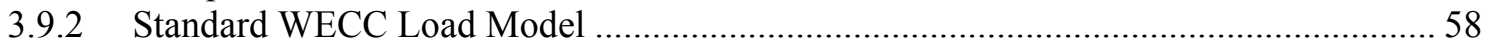

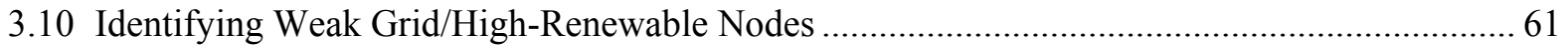

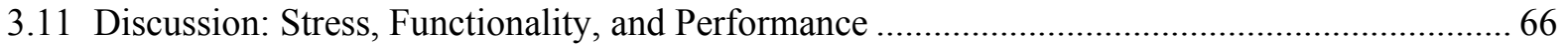

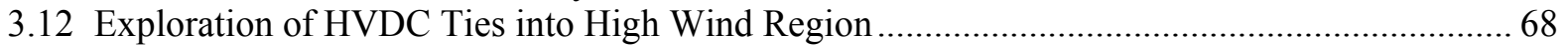

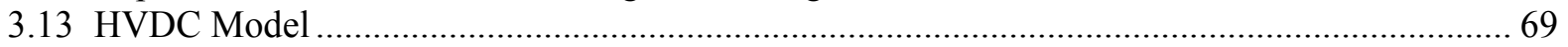

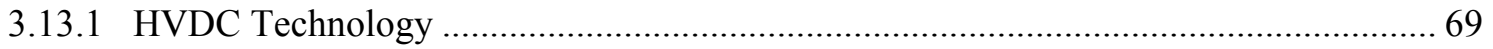

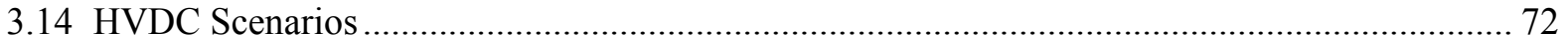

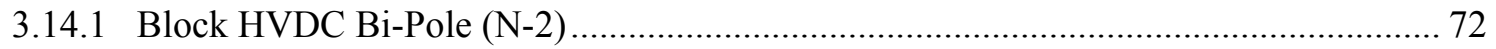

3.14.2 N-1-1 with No Repositioning or Remedial Action Scheme …….................................. 75

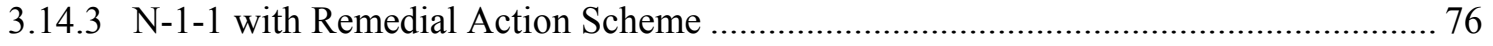

3.14.4 System Repositioning After Loss of HVDC Link ................................................. 78

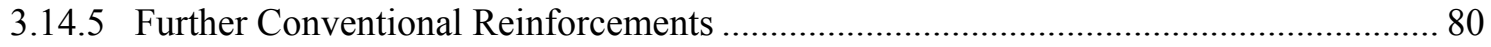

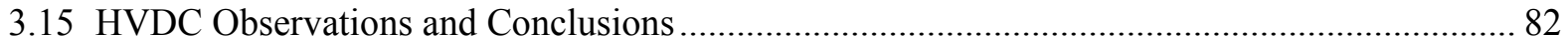

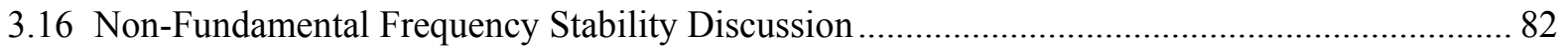




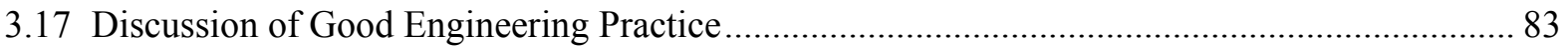

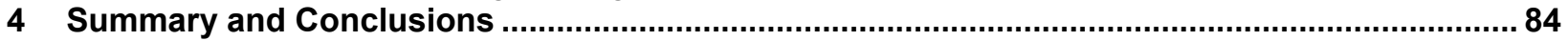

4.1 Key Observations on Low Levels of Synchronous Generation ........................................... 84

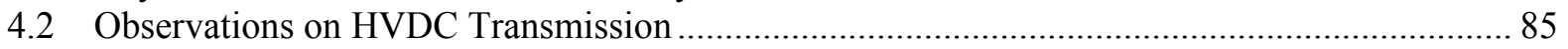

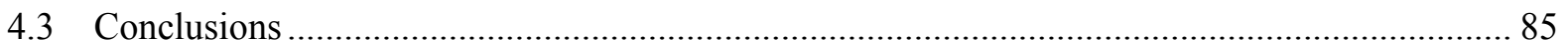

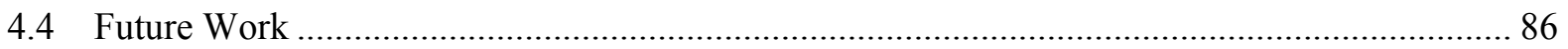

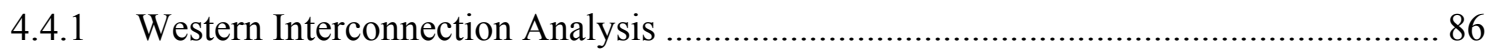

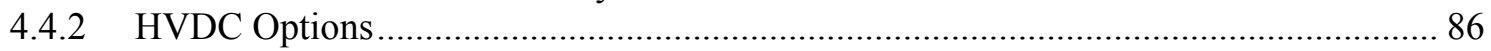

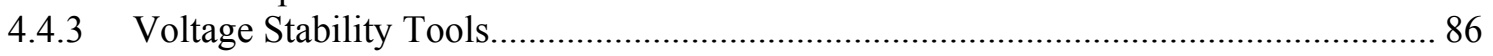

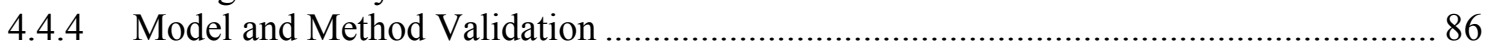

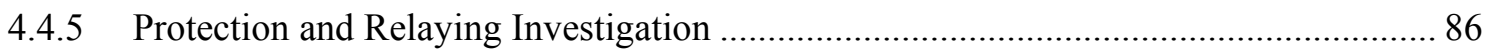

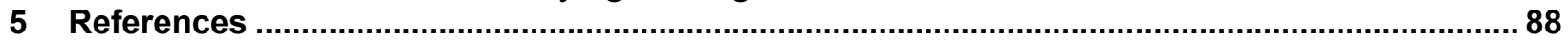




\section{List of Figures}

Figure ES-1. Visualization of transient stability with synchronous generators and wind power plants ..... vii

Figure ES-2. Small inverter-based power plant relative to grid............................................................. viii

Figure ES-3. Large inverter-based power plant relative to grid............................................................... viii

Figure ES-4. Penetration duration curve from WWSIS-2 production simulations ...................................

Figure ES-5. Wind and solar generation in the Light Spring Hi-Mix case ............................................. xi

Figure ES-6. Wind and solar generation in the Light Spring Extreme case ......................................... xii

Figure 1. Visualization of transient stability with synchronous generators and wind power plants ............. 4

Figure 2. Substation voltage in response to a transmission system disturbance ....................................... 5

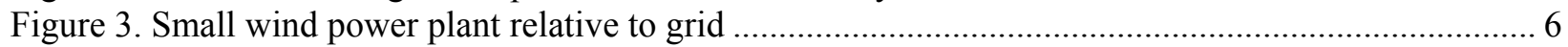

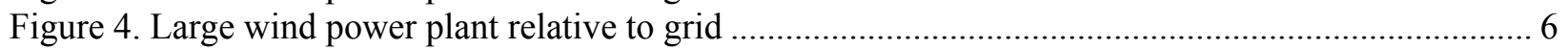

Figure 5. Penetration duration curve from WWSIS-2 production simulations ......................................... 8

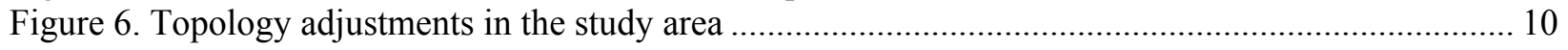

Figure 7. Wind and solar generation in the Light Spring Hi-Mix case .................................................. 12

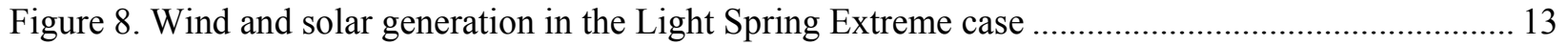

Figure 9. Dispatch details in the Desert Southwest and Northeast regions............................................ 14

Figure 10. Flow details in the vicinity of the future Aeolus substation ............................................... 15

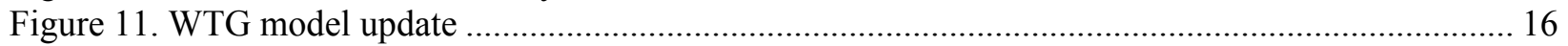

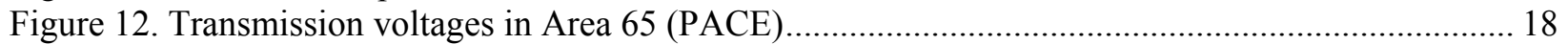

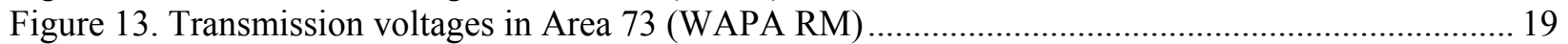

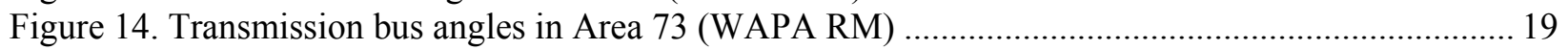

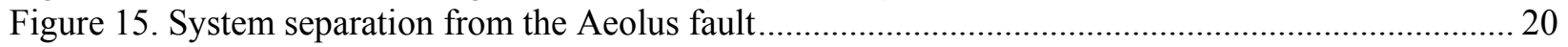

Figure 16. Active power from wind power plants in Area 73 (WAPA RM) ........................................ 21

Figure 17. Active current for wind power plants in Area 73 (WAPA RM) ............................................ 22

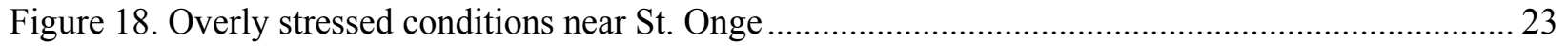

Figure 19. Model of fast control (LVPL) for power reduction during a fault.......................................... 24

Figure 20. Bus voltages in Area 73 (WAPA RM) as impacted by LVPL ........................................... 25

Figure 21. Wind power plant output during fault collapse arrested by LVPL ......................................25

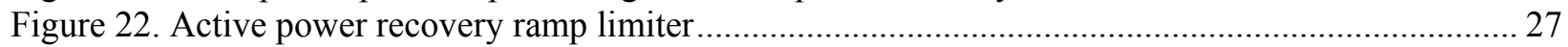

Figure 23. Post-fault oscillation-Area 73 (WAPA RM) voltages ....................................................2 28

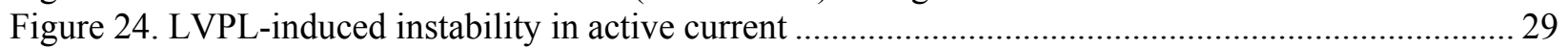

Figure 25. LVPL-induced instability observed at the WTG terminal voltages ...................................... 29

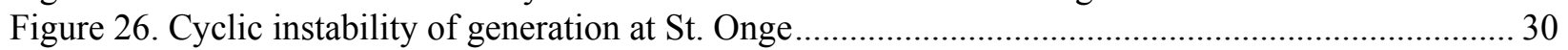

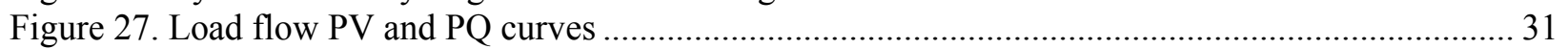

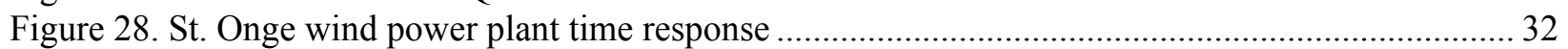

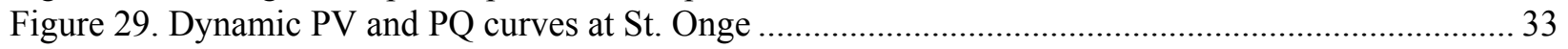

Figure 30. Dynamic voltage stability limit theory. Image from J.H. Chow 1989 ...................................... 35

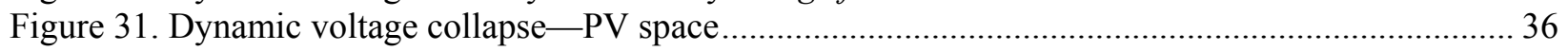

Figure 32. Phase portrait showing the limit cycle on a WTG at St. Onge ............................................. 37

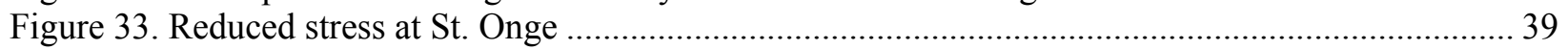

Figure 34. Reduced stress but limited LVPL— bus voltages in Area 73 (WAPA RM) .......................... 40

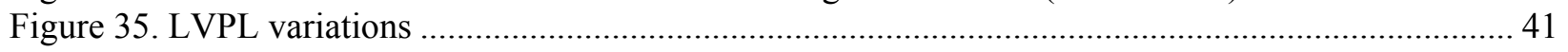

Figure 36. Voltages in Area 73 (WAPA RM) for reduced stress with aggressive LVPL........................ 42

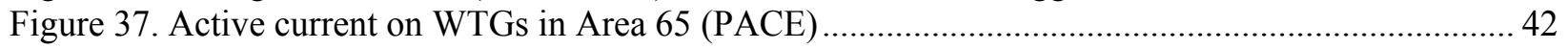

Figure 38. Performance of St. Onge wind power plant for reduced stress case ....................................... 43

Figure 39. Less aggressive LVPL for the reduced stress extreme case ................................................ 44

Figure 40. Less aggressive LVLP — reactive response from WTGs in Area 73 (WAPA RM)................. 44

Figure 41. Synchronous generator at St. Onge — extreme dispatch ...................................................... 45

Figure 42. Area 73 (WAPA RM) bus voltages with synchronous generator at St. Onge ........................ 46

Figure 43. Synchronous machine speeds in Area 73 (WAPA RM) for the synchronous St. Onge case .... 46 
Figure 44. St. Onge synchronous machine response — reduced dispatch case....................................... 47

Figure 45. Voltage swing comparison: WTG (top) compared to synchronous generator (bottom) ........... 48

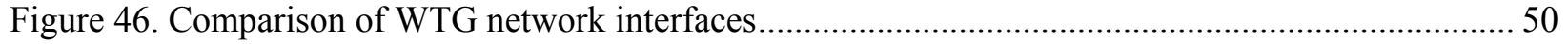

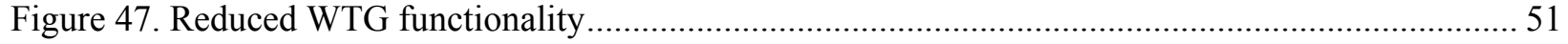

Figure 48. Area 73 (WAPA RM) voltage for Extreme case - OEM model ............................................ 52

Figure 49. Area 73 (WAPA RM) terminal voltages (Vt) for Extreme case-OEM model.......................5 52

Figure 50. Area 73 (WAPA RM) active power-OEM model …......................................................... 53

Figure 51. Area 73 (WAPA RM) reactive power-OEM model ....................................................... 53

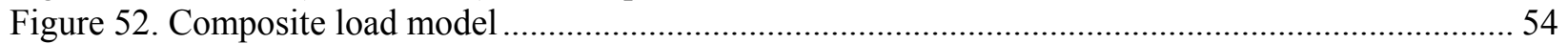

Figure 53. Voltages — composite load with motor tripping (detail bottom) ........................................... 55

Figure 54. Voltage at load bus for a composite load with motor tripping ............................................ 56

Figure 55. Details of active power composite load response with motor tripping..................................5 57

Figure 56. Details of reactive power response with motor tripping ........................................................5

Figure 57. Active current response for WTGs in Area 73 (WAPA RM) ...............................................5

Figure 58. Reduced stress case with (old) standard WECC load model .................................................59

Figure 59. Dynamic PV for St. Onge wind power plant with standard WECC load model ...................... 60

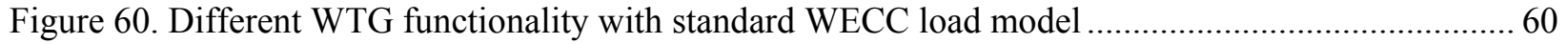

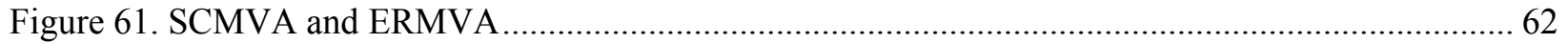

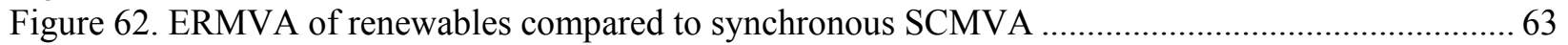

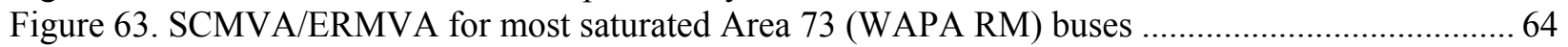

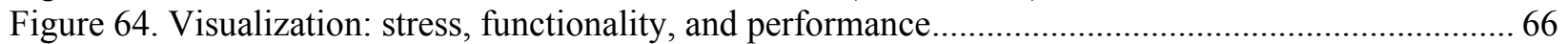

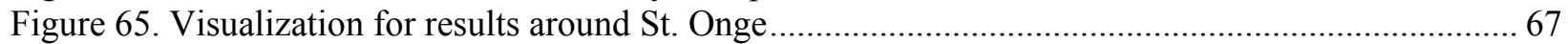

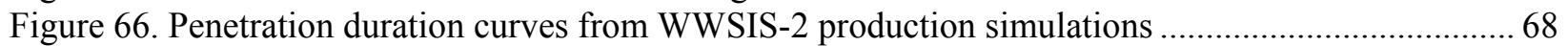

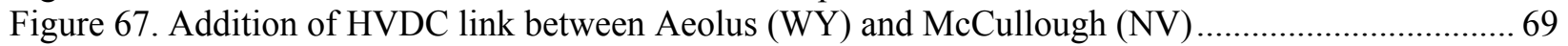

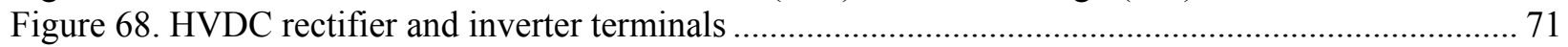

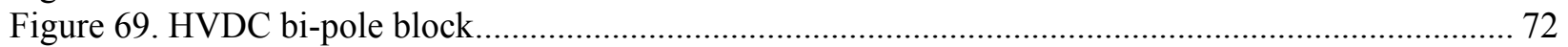

Figure 70. Area 65 (PACE) bus voltage dynamics for the HVDC bi-pole block ................................... 73

Figure 71. Effect of HVDC block on reactive power generation in Area 65 (PACE) ............................. 74

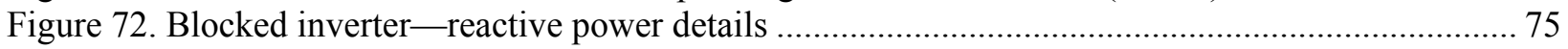

Figure 73. Voltage with HVDC already blocked (N-1), trip Gateway West (N-1-1) ............................. 76

Figure 74. Voltage collapse with RAS trip of $600 \mathrm{MW}$ of wind at Snowy Range ................................... 77

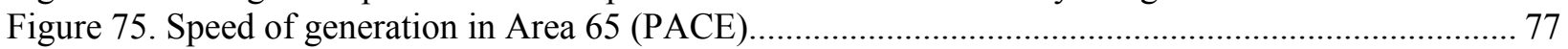

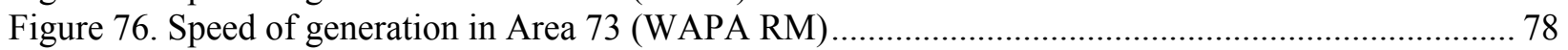

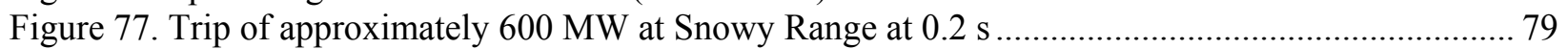

Figure 78. Gateway West trip with 600 MW RAS and repositioned system........................................... 80

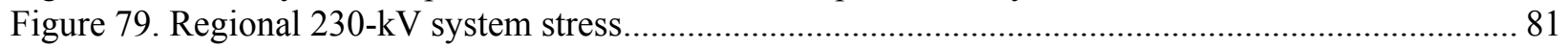

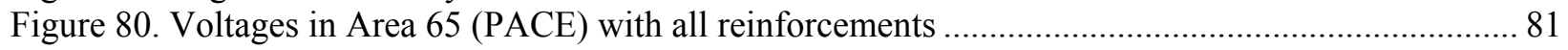

\section{List of Tables}

Table ES-1. Wind and Solar Generation in the Light Spring Hi-Mix Case .............................................. xi

Table ES-2. Wind and Solar Generation in the Light Spring Extreme Case ..........................................

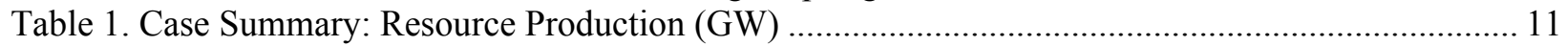

Table 2. Wind and Solar Generation in the Light Spring Hi-Mix Case ................................................ 12

Table 3. Wind and Solar Generation in the Light Spring Extreme Case ............................................... 13

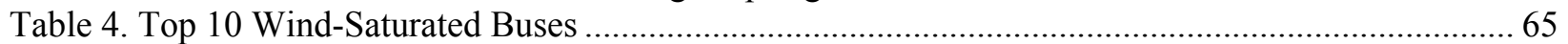




\section{Introduction}

The stability of the North American electric power grids under conditions of high penetrations of wind and solar is a significant concern and possible impediment to reaching renewable energy goals. The 33\% wind and solar annual energy penetration considered in this study results in substantial changes to the characteristics of the bulk power system. This includes different power flow patterns, different commitment and dispatch of existing synchronous generation, and different dynamic behavior from wind and solar generation.

The investigation reported in this document builds on the foundation of the different phases of the Western Wind and Solar Integration Study (WWSIS) described below. The specific focus of this work is on the transient stability of systems in which wind generation has displaced synchronous thermal generation under highly stressed, weak system conditions.

Transient stability, the ability of the power system to maintain synchronism among all elements following disturbances, is a major constraint on operations in many grids, including the western U.S. and Texas systems. These constraints primarily concern the performance of the large-scale bulk power system, because local transient stability concerns are generally addressed on the level of individual plants. But grid-wide stability concerns with high penetrations of wind and solar are still not thoroughly understood. Phase 3 of WWSIS (WWSIS-3) included significant transient stability work. That work highlighted the need for additional investigations and provided the basis for this study. This work focuses on "traditional" fundamental frequency stability issues, such as maintaining synchronism, frequency, and voltage. This work does not explore non-fundamental frequency issues, such as subsynchronous phenomena, harmonics, unbalances, transients, and small-signal analysis.

The objectives of this study are to better understand the implications of low levels of synchronous generation and a weak grid on overall system performance by:

- Investigating the Western Interconnection under conditions of both high renewable generation (e.g., wind and solar) and low synchronous generation (e.g., significant coal power plant decommitment or retirement).

- Analyzing both the large-scale stability of the Western Interconnection and regional stability issues driven by more geographically dispersed renewable generation interacting with a transmission grid that evolved with large, central station plants at key nodes.

\subsection{Background and Related Work}

WWSIS, sponsored by the U.S. Department of Energy, is one of the largest regional solar and wind integration studies to date. In multiple phases, it has explored different aspects of the question: Can we integrate large amounts of wind and solar energy into the electric power system of the West? The work reported here focused on the impact of low levels of synchronous generation caused by high penetrations of wind and solar on the transient stability performance in one part of the region. It is essentially an extension of WWSIS-3. An overview of the WWSIS research program and recommendations for further reading are provided below. 


\subsubsection{WWSIS-1}

The first phase of WWSIS (GE Energy 2010a, GE Energy 2010b) investigated the benefits and challenges of integrating up to $35 \%$ wind and solar energy in the WestConnect subregion and, more broadly, the Western Interconnection in 2017. The study showed that it is operationally feasible to accommodate 30\% wind and 5\% solar energy if utilities substantially increase their coordination of operations throughout wider geographic areas and schedule their generation and interchanges on an intra-hour basis. Key findings included:

- The integration of 35\% wind and solar energy into the electric power system will not require extensive additional infrastructure if changes are made to operational practices.

- Wind and solar energy displace fossil fuels. Therefore, a 35\% penetration of solar and wind power would reduce fuel costs by $40 \%$ and carbon emissions by $25 \%-45 \%$.

- Increasing the size of the geographic area throughout which the wind and solar resources are drawn substantially reduces relative variability.

- Scheduling generation and interchanges subhourly reduces the need for fast reserves.

- Using wind and solar forecasts in utility operations reduces operating costs by up to $14 \%$.

- Existing transmission capacity can be better used, which would reduce new transmission needs.

- Demand response programs can provide flexibility that enables the electric power system to more easily integrate wind and solar - and may be cheaper than generation alternatives.

\subsubsection{WWSIS-2}

Phase 2 of WWSIS was initiated to determine the wear-and-tear costs and emissions impacts of cycling and to simulate grid operations to investigate the detailed impacts of wind and solar power on the fossil-fueled fleet in the West (Lew and Brinkman 2013, Lew et al. 2013). Key findings included:

- Wind- and solar-induced cycling can have a small positive or negative impact on emissions rates, depending on the pollutant and wind and mix of wind and solar. Any adverse impact of cycling on overall plant emissions is relatively small: $1 \%-5 \%$, depending on the study scenario.

- Any increase in plant emissions from cycling to accommodate variable renewables is more than offset by the overall reduction in carbon dioxide, nitrogen oxide, and sulfur dioxide. In the high wind and solar scenario, net carbon emissions were reduced by onethird.

- Annual cycling costs increase by \$35-\$157 million for fossil-fueled plants when high penetrations of variable renewables are added to the electric grid. This represents an additional $\$ 0.47 / \mathrm{MWh}-\$ 1.28 / \mathrm{MWh}$ of cycling costs for the average fossil-fueled generator. Starts, rather than ramps, drive the cycling costs, and gas combustion turbines 
see the highest wear-and-tear costs. From a system perspective, these increased costs are relatively small compared to the $\$ 7$ billion in fuel savings associated with wind and solar generation.

- The price of gas has a much greater impact on system-wide cycling costs than the addition of wind and solar.

- At high penetrations, solar dominates variability on both a subhourly and hourly basis, with the extreme events driven by sunrise and sunset. At a system level, however, most of this variability comes from the known path of the sun instead of from fast-moving clouds.

- At high penetrations, wind dominates uncertainty. It leads to greater extremes in the dayahead forecast error. Because 4-hour-ahead forecasts are much more accurate, a 4-hourahead unit commitment can mitigate this uncertainty.

\subsubsection{WWSIS-3}

Phase 3 of WWSIS delved into the dynamic performance of the grid in the fractions of a second to 1 min following a large disturbance (e.g., loss of a large power plant or a major transmission line), which is critical to system reliability. In particular, this study examined the large-scale transient stability and frequency response of the Western Interconnection with high penetrations of wind and solar, and it identified means to mitigate any adverse performance impacts via transmission reinforcements, storage, advanced control capabilities, or other alternatives (Miller et al. 2014a, Miller et al. 2014b). Key findings included:

- With good system planning, sound engineering practices, and commercially available technologies, the Western Interconnection can withstand the crucial first minute after grid disturbances with high penetrations of wind and solar.

- Local stability, voltage, and thermal problems can be addressed with traditional transmission system reinforcements (e.g., transformers, shunt capacitors, local lines).

- Nontraditional frequency-responsive controls on wind, utility-scale solar photovoltaic power (PV), concentrating solar thermal power (CSP), and energy storage are effective at improving frequency response after the loss of approximately $2,750 \mathrm{MW}$ of central station generation.

- Load-modeling assumptions can have as much impact on system performance as high penetrations of wind and solar. Accurate modeling of load, as well as renewable generation, is extremely important when analyzing high-stress conditions.

As noted above, the work reported here is an extension of the research performed in WWSIS-3.

\subsubsection{Transient Stability Discussion}

In addition to maintaining the balance between electricity generation and electricity demand, power system operators must ensure that the grid can successfully transition from normal operation (e.g., all transmission lines and generating units are in service), through a disturbance (e.g., an abrupt outage of a major transmission line or large generator), and into a new stable operating condition in the 10-20 s immediately following a disturbance. The ability to make this successful transition is called transient stability. 
Figure 1 shows an updated visualization of the problem originally presented in (Vittal 2003) to include wind and PV. The round masses represent generators, with the tension on the various springy lines representing power transfer. The board at the top represents the simplified idea of an infinite bus - a real, finite power system is floating. The level at which it is floating is a proxy for frequency, which must stay close to $60 \mathrm{~Hz}$. The hands represent wind and PV. They put tension (inject power) on the system, but they are all control and not weight. The mission of these devices, unless taught to do otherwise, is to pull uniformly, regardless of whether the node to which they are connected is moving or not.

The scissors represent a disturbance, which might cut a line or disconnect a generator. The rubbery mass-spring system bounces around. If the event is too severe or some of the lines are stretched too taut (too much loading), more lines will break. It is easy to imagine a cascading failure in which each successive break leads to another failure. A substantial part of system planning is aimed at avoiding such unacceptable consequences.

Much of the investigation of WWSIS-3 explored different ways to make the controls of wind and PV generators (the hands in the drawing) smarter for maintaining frequency. That work showed that available controls for wind power plants are effective at improving system performance.

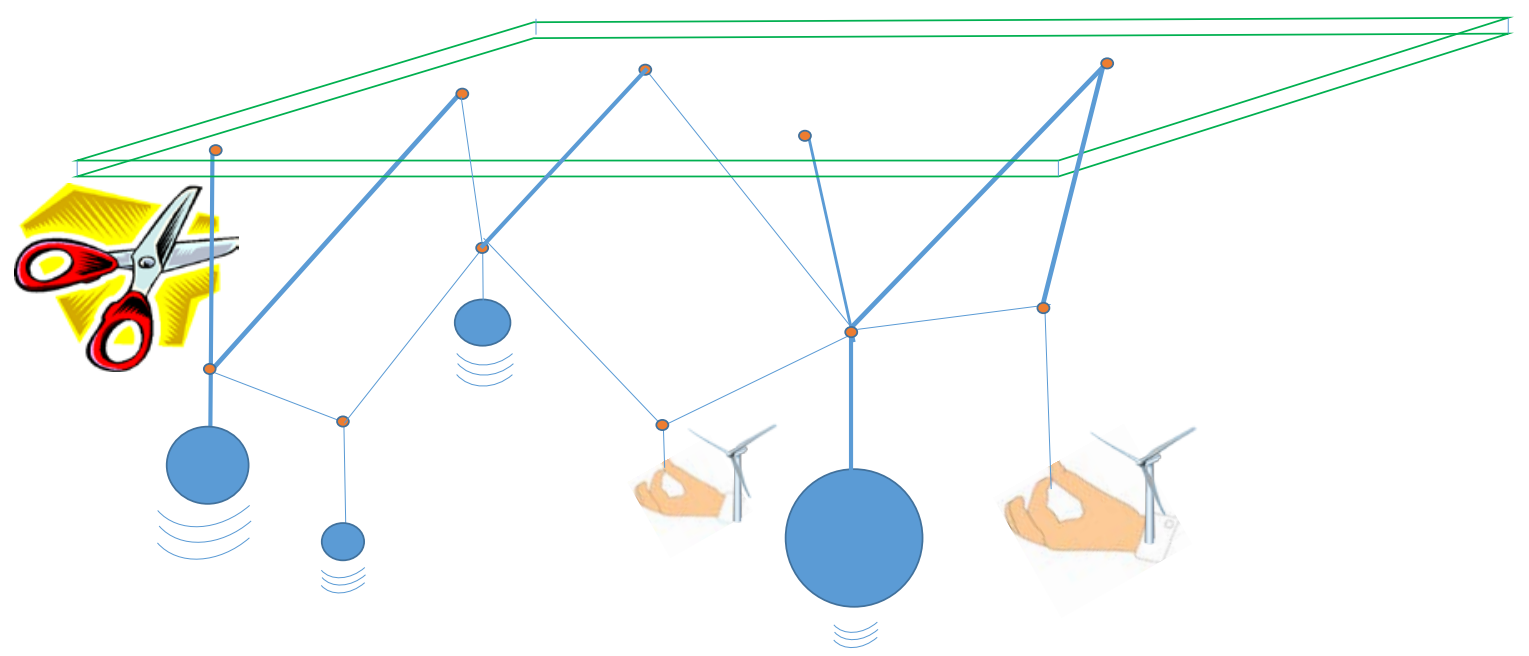

Figure 1. Visualization of transient stability with synchronous generators and wind power plants

An example of both transient stability (blue) and transient instability (red) is shown in Figure 2. The system is operating normally, with a transmission substation voltage of $100 \%$, up to $0.5 \mathrm{~s}$. At that time, a disturbance occurs, such as a tree falling on a transmission line. From $0.5-0.7 \mathrm{~s}$, the voltage is zero because the tree is connecting the transmission line to the ground. At approximately $0.7 \mathrm{~s}$, an automatic protection system trips the transmission line, and the voltage returns to near normal. But, as described above, the system is swinging in response to the disturbance. When the swings grow and the system separates, the substation voltage drops precipitously and the system collapses at approximately $1 \mathrm{~s}$. When the swings die down, the substation voltage settles back to normal within $5 \mathrm{~s}$. This study focuses on system stability in the first $5-10 \mathrm{~s}$. 


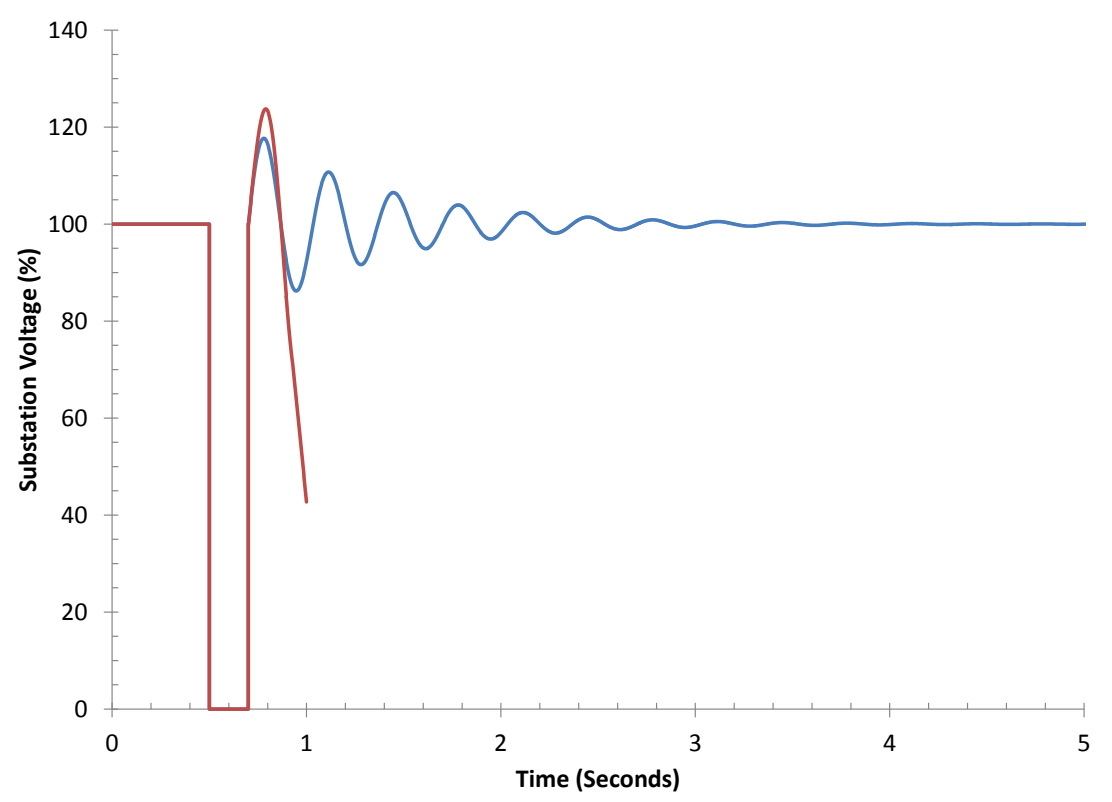

Figure 2. Substation voltage in response to a transmission system disturbance

The Western Interconnection has a long history of constraints because of transient stability limitations that vary depending on system characteristics such as the level of electricity demand (e.g., peak summer load), the amount of power flowing on the transmission system (e.g., heavy flows on critical paths), and the location of the generating plants in operation (e.g., remote from population centers). The primary measures of transient stability are to avoid bulk system separation and individual generator loss of synchronism with the system and to meet various voltage and frequency swing criteria that vary with the severity of the disturbance according to reliability standards set by the North American Electric Reliability Corporation and Western Electricity Coordinating Council (WECC 2011). Transient stability can be both systemic and local. Large penetrations of inverter-based, or nonsynchronous, wind and solar generation may substantially alter system stability as a result of changes in angle/speed swing behavior caused by reduced inertia, changes in voltage swing behavior because of different voltage control systems, different power flow patterns, and the displacement of synchronous generation at key locations.

\subsubsection{Weak Grid Description}

One of the most challenging operating conditions for inverter-based generation is in a so-called weak grid. But what is a weak grid? Analytical descriptions have long been available (Tande and Olav 2000), but in essence, the question is one of relative size: Is the inverter-based generation relatively small or relatively large compared to the host grid?

Historically, wind power plants were relatively small compared to the grid. A 1-MW wind turbine connected to a 20-GW system has no more ability to move that system than a lap dog, even a badly behaved one, can bend a palm tree (Figure 3). However, as wind power plants became larger and are connected into smaller and more remote portions of the grid, they exert more influence. A 200-MW wind power plant connected to a 300-MW subsystem can now impact system response to a disturbance similar to the way in which an elephant can bend or break a palm tree unless it is trained not to (Figure 4). 
It is this latter condition of high penetrations of wind and low levels of synchronous generation in a relatively small and remote part of the Western Interconnection that is the focus of this report.

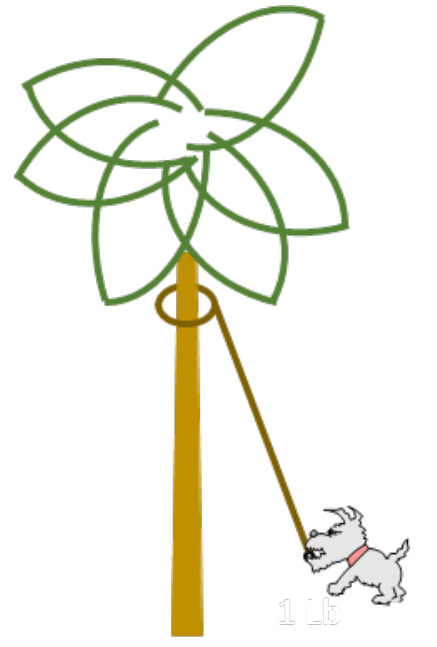

Figure 3. Small wind power plant relative to grid

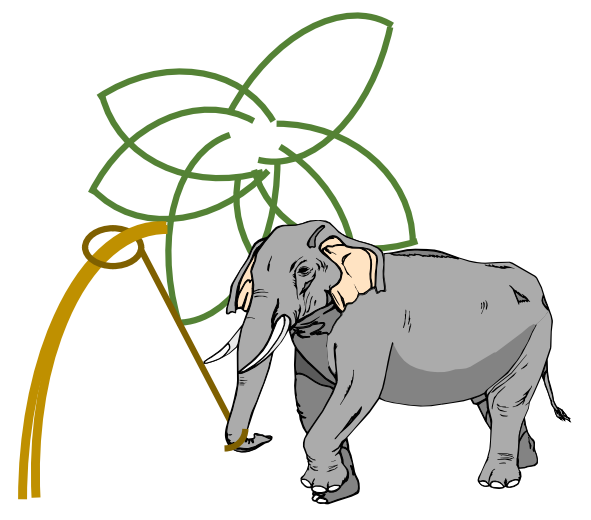

Figure 4. Large wind power plant relative to grid 


\section{Database Refinement}

The database refinement for this work focused on incremental updates to the WWSIS-3 databases rather than on developing a substantially different wind and solar penetration level, or new conventional generation commitment and dispatch derived from the WWSIS-2 PLEXOS results, or starting from a different initial WECC power flow and dynamic data set. Selecting a new study scenario and creating a brand-new database were not included in the scope of work for this project.

The database refinement for this study was intended to bring the Light Spring cases from WWSIS-3 into closer alignment with today's consensus of what the system would look like in 2022. Inputs from the technical review committee were collected to determine any necessary changes to the generation mix or transmission topology. These changes were implemented in all three cases:

- $\quad$ Light Spring Base

- $\quad$ Light Spring Hi-Mix

- Light Spring Extreme

The Light Spring Base case represents a future in which the current renewable portfolio standard targets are met. The Hi-Mix case was built form the Base case but includes even higher levels of wind and solar generation. It represents a snapshot in time: a windy, sunny morning in the spring. The details (e.g., renewable plant MW output, conventional generation redispatch/decommitment) were mined from WWSIS-2 and are reported in Miller et al. (2014a, 2014b). The Light Spring Extreme case has an even higher penetration of wind and solar generation. Both the Hi-Mix and Extreme cases have the same "high renewable" topology-i.e., the same plants at the same buses with the same ratings. The differences are in the wind and solar power plant outputs and the associated redispatch and decommitment of the rest of the generation fleet. The Extreme case represents the highest total wind plus solar production sample of all windy, sunny mornings in the spring data.

The selection of the initial condition for the stability analysis was a key consideration. In the WWSIS-3 study process, lengthy discussions were held regarding which conditions should be examined. Some of that decision-making process is recorded in the study report, but it is useful to provide some context here. To evaluate the impact on transient stability and frequency response of high levels of wind and solar generation, it is useful to select conditions in which the penetration levels of these resources are high. Further, it is well known that light load conditions represent some of the more challenging conditions, especially for frequency response. The California Independent System Operator and others are particularly worried about light load in the spring, when there is a high level of hydropower production. Many plants are at maximum dispatch and cannot maintain additional headroom without spilling water. Thus, light spring conditions with high levels of wind and solar are of particular interest, since hydro at maximum cannot provide primary frequency response. In addition, they may not be dispatchable without violating one of the many non-energy production constraints on the hydro system (e.g., irrigation, recreation, wildlife management). Because it must be daytime for there to be solar 
generation, such light load conditions (e.g., a sunny, windy weekend morning) are not the absolute minimum load condition. That is likely to happen in the early, pre-sunrise hours of the morning. But there will only be wind generation then, so the maximum instantaneous penetration for this mix of variable renewable generation is expected to be lower.

Figure 5 shows the generation penetration duration curves for the original WWSIS-2 production simulation Hi-Mix case (blue), and Base case (red). The curves show 5-min resolution for the study year, so there are slightly more than 100,000 samples per year. Instantaneous generation penetration for all of WECC (not only the U.S. portion) for the data from the three study cases, Base, Hi-Mix and Extreme, are pointed out in the figure. The Base and Hi-Mix cases are in the top $10 \%$ of hours for the year - the difference in penetration is due to the difference in installed wind and solar generation. The extreme case is in the top $<1 / 2 \%$ - the difference between the HiMix and Extreme cases is due to higher speed wind and higher intensity solar on the same installed base of equipment.

Although these cases were expected to be both challenging and illuminating for this investigation, there is no implication that these cases are necessarily the most difficult in all regards. For example, autumn light load, with less hydropower, is known to be difficult for PacifiCorp. As stated elsewhere, this investigation is not a substitute for the complete, detailed planning analysis that must accompany a massive evolution of a system.

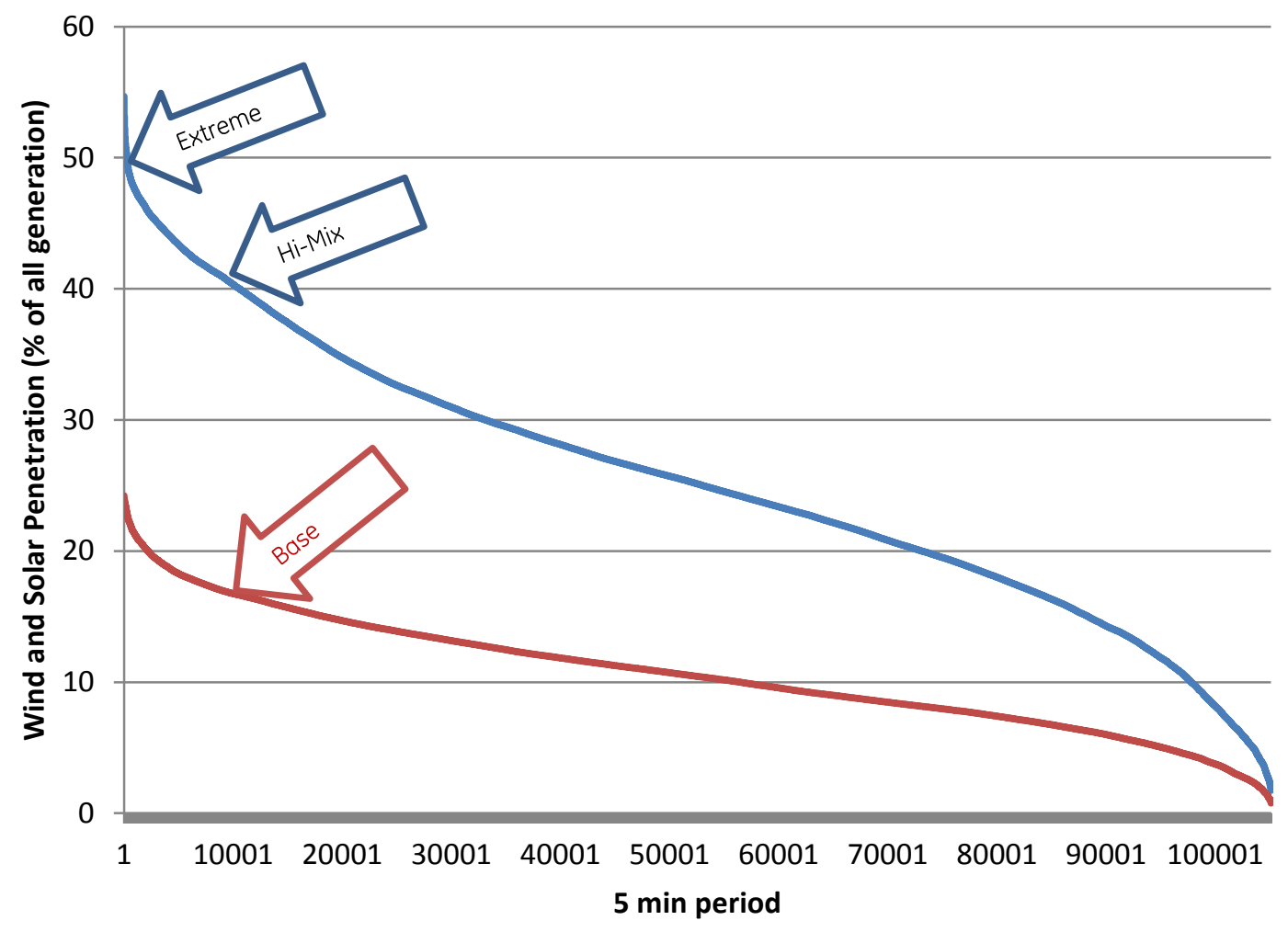

Figure 5. Penetration duration curve from WWSIS-2 production simulations 


\subsection{Transmission Topology Refinements}

This project did not have the option to start with completely new databases. Rather, the intent was to capture major transmission projects that are likely to impact the transient stability of the system, particularly in the focus area. As such, focus was directed particularly at changes in the northeast and southeast side of the system. There was no intent to get exact topologies; this investigation is not a substitute for thorough system planning studies.

Based on inputs from the technical review committee, a number of updates to the light spring transmission topology were made to the three power flow cases. These updates included:

- Gateway South in

- Gateway West in

- Phoenix-Sun Valley-Palo Verde in

- Pawnee-Daniels Park-Smoky Hill in

- Robinson Summit-Midpoint out

- Four Corners-West Mesa-Moenkopi-Las Vegas out

- SunZia East-SunZia South-Orosburg-Phoenix out

- Afton-Hidalgo out

- Comanche-Calumet-San Luis Valley out

The WECC facilities map shown in Figure 6 includes annotations reflecting the bulk transmission assumptions that were key to this study.

A number of other local reinforcements were carried forward from WWSIS 3. These were additions to the Light Spring Base that allowed the Hi-Mix or Extreme case to avoid severe overloads or local voltage problems. These transmission improvements were made in the narrow context of relieving local thermal and voltage problems, and they were emphatically not the result of a larger transmission expansion planning exercise. Clearly, such detailed planning would be necessary as actual wind and solar projects are proposed, but it is not particularly meaningful for this investigation. Not every overload was completely eliminated, a point that will be examined further in the next section. Improvements included:

- 230-kV segments added/doubled

- Marias to Marias N

○ Eagle Eye-Liberty

○ Gilariver-Gilabend

○ N Yuma-Story

○ N Park-Hayden

○ Terry Ranch-Cheyenne 
○ Cheyenne-Snowy Range (Two additional circuits, for a total of three, were added between the Hi-Mix and Extreme case; this particular reinforcement is revisited in the section on high-voltage direct current [HVDC] below.)

- 230-/500-kV transformers added/doubled

○ Gilariver

○ Wilsall

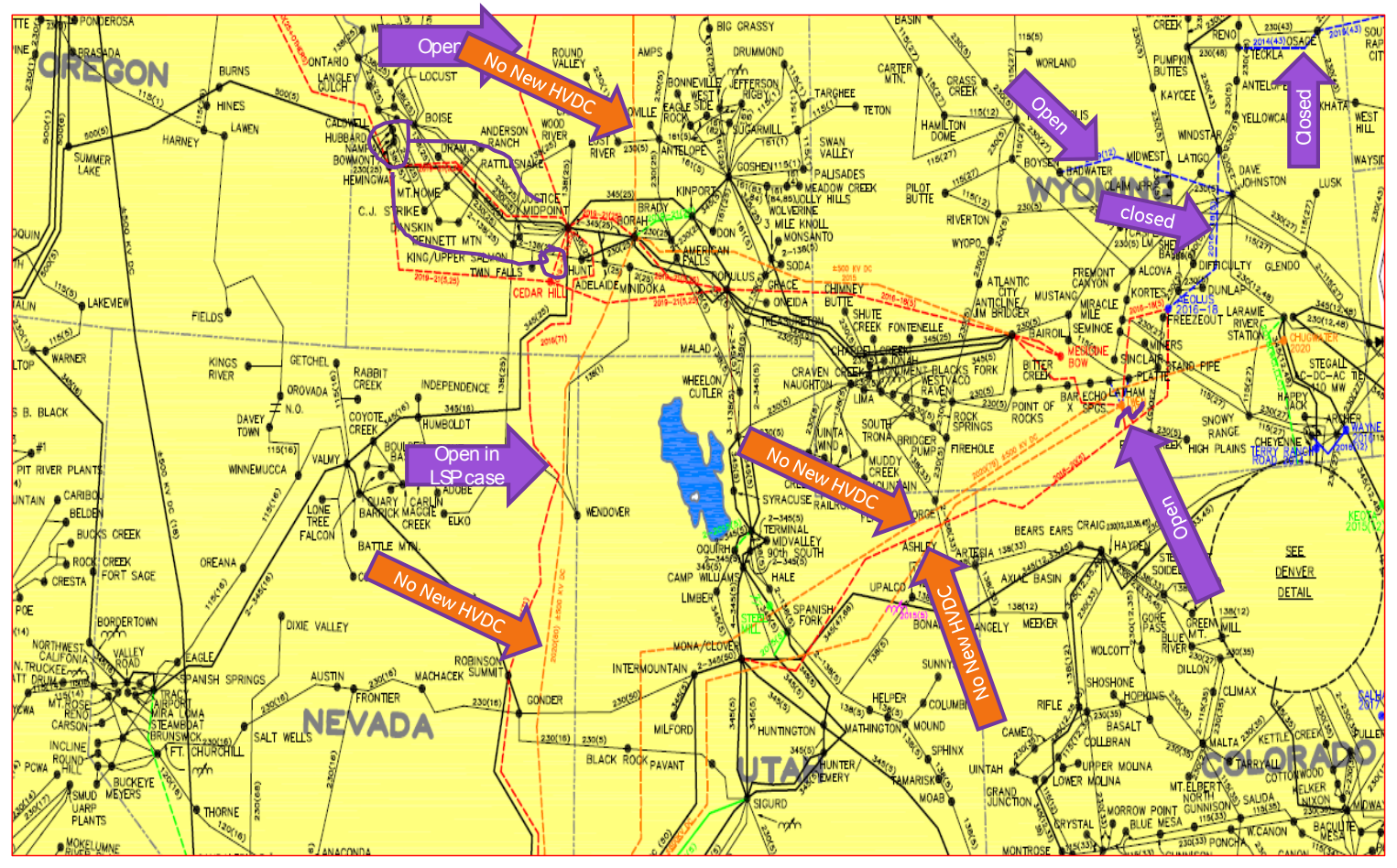

Figure 6. Topology adjustments in the study area

\subsection{Utility-Scale PV Replacement of Low-Probability CSP}

The Light Spring Base case includes approximately 900 MW of CSP plants. The Hi-Mix case from WWSIS-3 increases that by $7.5 \mathrm{GW}$ to approximately $8.4 \mathrm{GW}$. However, since the development of these cases for WWSIS-3, the market for CSP in the western United States has largely disappeared. Many proposed CSP projects have been converted to utility-scale PV projects. For this study, all the CSP in the Light Spring Base case was retained and matched to the CSP database from the National Renewable Energy Laboratory (NREL) when possible. ${ }^{3}$ Then NREL and other data were used to identify high-probability CSP plants that were under construction or fully financed. Five CSP plants (Ivanpah, Mojave, Tonopah, Genesis, and Solana) were identified. New CSP plants in the Hi-Mix case that roughly aligned with these projects were retained. Thus, $3.3 \mathrm{GW}$ of CSP were retained in the Hi-Mix case for this study. All other CSP plants were converted to utility-scale PV. The net result was that 70 of 81 plants totaling approximately 5,100 MW were converted from CSP to utility-scale PV.

\footnotetext{
${ }^{3}$ Thank you to Mark Mehos at NREL for his support of this work and supplying the source material/CSP database.
} 


\subsection{Light Spring Dispatch}

A high-level summary of the wind and solar dispatch for the three light spring cases is given in Table 1. The Hi-Mix and Extreme cases include the conversion of CSP to utility-scale PV. Totals and penetration figures are for the U.S. portion of WECC only. For a quick comparison, the U.S. wind and solar production totals in the three cases are as follows:

- Approximately $24.0 \mathrm{GW}$ in the Base case

- Approximately 55.9 GW in the Hi-Mix case

- Approximately $63.1 \mathrm{GW}$ in the Extreme case.

Table 1. Case Summary: Resource Production (GW)

\begin{tabular}{llll} 
& Base Case & High Mix & Extreme \\
\hline Wind (GW) & 19.2 & 25.5 & 30.9 \\
PV (GW) & 3.9 & 17.5 & 20.4 \\
CSP (GW) & 0.9 & 1.2 & 1.4 \\
DG $^{a}(G W)$ & 0.00 & 11.7 & 10.1 \\
Others (GW) & 71.7 & 43.6 & 34.9 \\
\hline Total (GW) & 95.7 & 99.4 & 98.1 \\
\hline Penetration & $25.0 \%$ & $56.2 \%$ & $64.4 \%$ \\
\hline (\% of US generation dispatch) & & & \\
\hline
\end{tabular}

${ }^{\mathrm{a}}$ Distributed generation

The details of the difference between the Hi-Mix and Extreme PLEXOS samples are shown in the WWSIS-3 report and appendix. Summaries of the wind and solar generation in the Light Spring Hi-Mix and Extreme cases are shown below in Figure 7 and Table 2, and Figure 8 and Table 3, respectively.

\footnotetext{
${ }^{4}$ The penetration levels shown in this table were calculated considering only the U.S. portion of WECC generation.
} 


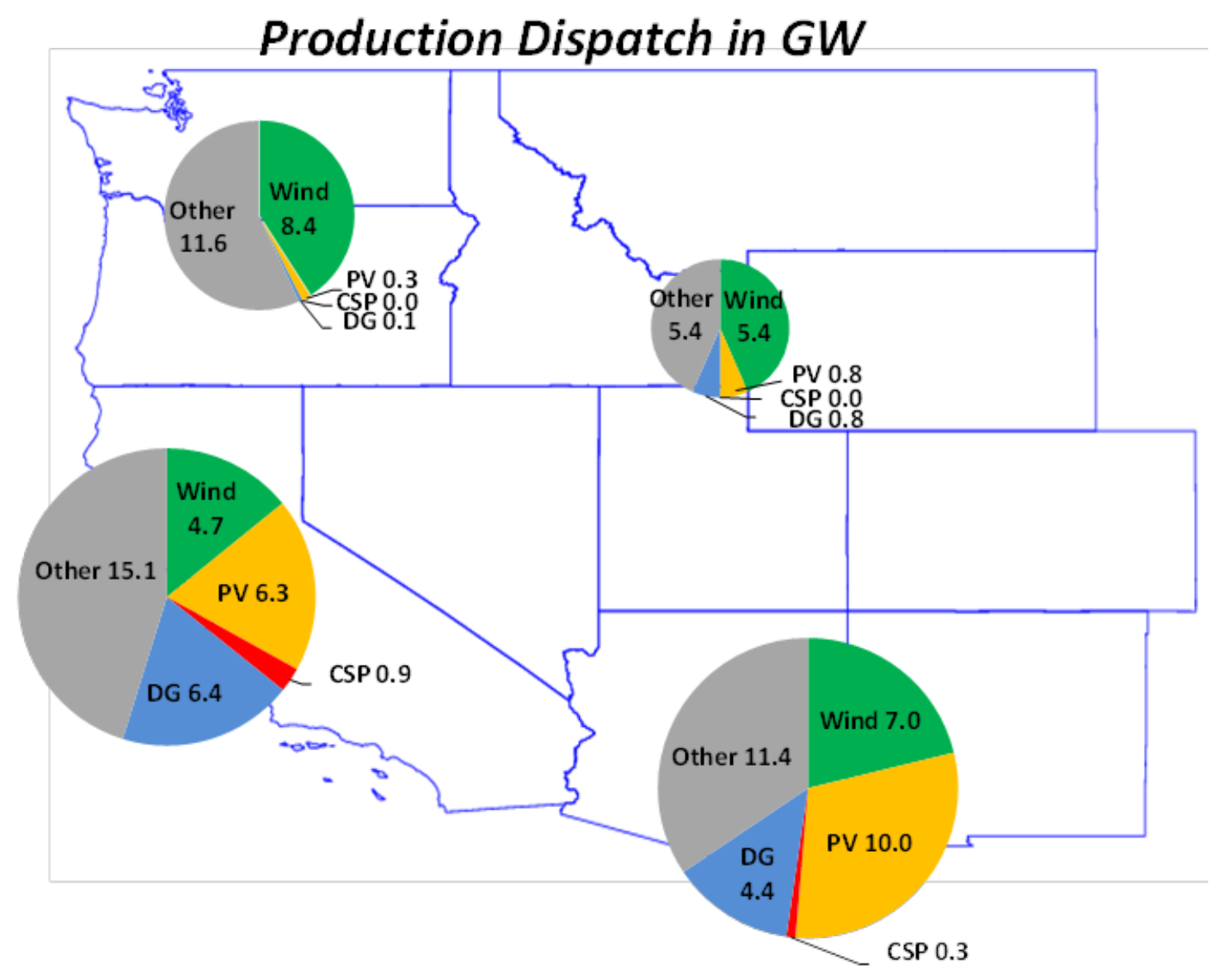

Figure 7. Wind and solar generation in the Light Spring Hi-Mix case

Table 2. Wind and Solar Generation in the Light Spring Hi-Mix Case

\begin{tabular}{llllll}
\hline & WECC (U.S.A.) & California & DSW & Northeast & Northwest \\
\hline Wind (GW) & 25.5 & 4.7 & 7.0 & 5.4 & 8.4 \\
PV (GW) & 17.4 & 6.3 & 10.0 & 0.8 & 0.3 \\
CSP (GW) & 1.2 & 0.9 & 0.3 & 0.0 & 0.0 \\
DG (GW) & 11.7 & 6.4 & 4.4 & 0.8 & 0.1 \\
Others (GW) & 43.6 & 15.1 & 11.4 & 5.4 & 11.6 \\
\hline Total (GW) & 99.5 & 33.5 & 33.1 & 12.4 & 20.4 \\
\hline Penetration & $56.2 \%$ & $54.8 \%$ & $65.6 \%$ & $56.6 \%$ & $43.0 \%$ \\
(\% of US generation dispatch) & & & & & \\
\hline
\end{tabular}

\footnotetext{
${ }^{5}$ The penetration levels shown in this table were calculated considering only the U.S. portion of WECC generation.
} 


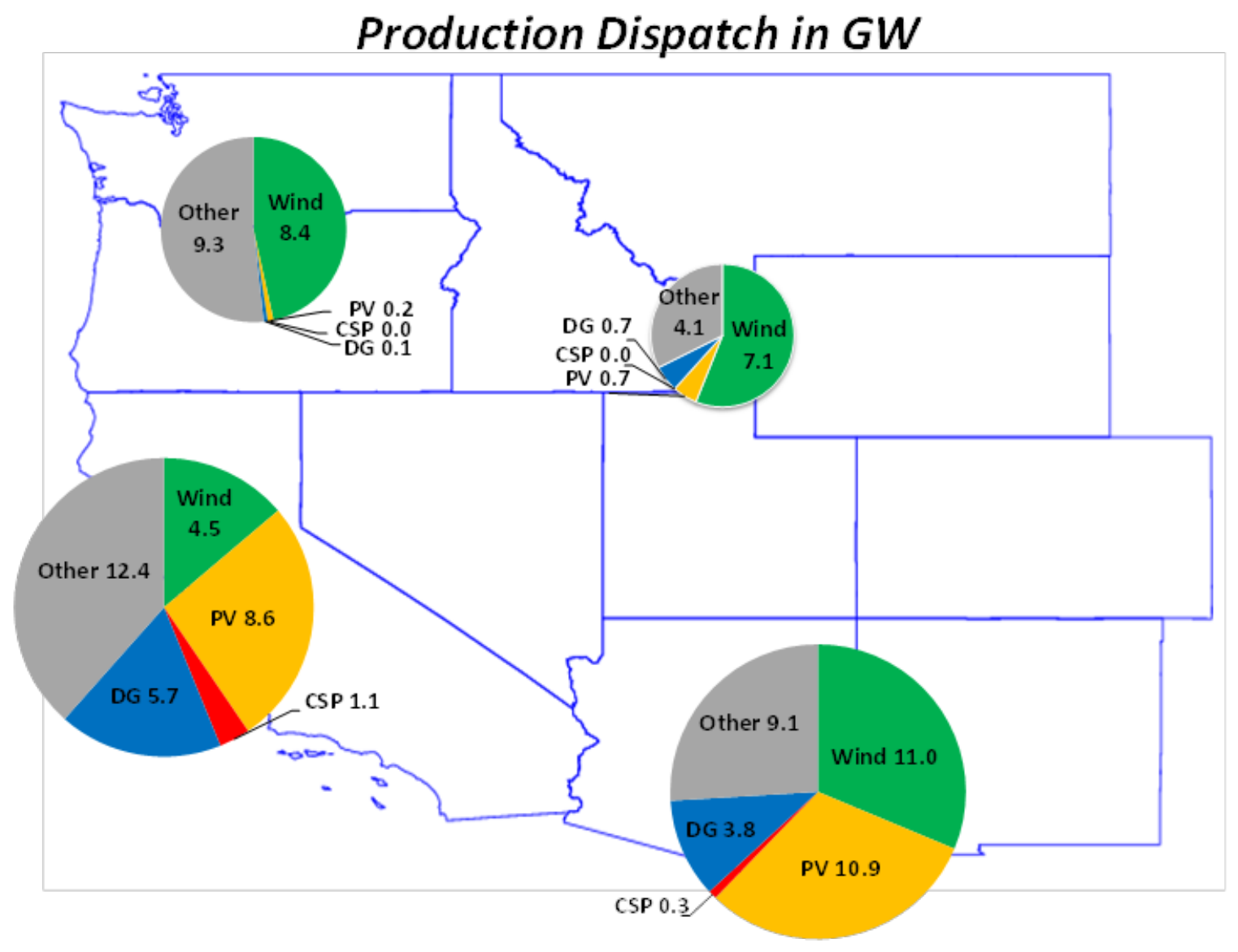

Figure 8. Wind and solar generation in the Light Spring Extreme case

Table 3. Wind and Solar Generation in the Light Spring Extreme Case

\begin{tabular}{llllll}
\hline & WECC (U.S.A.) & California & DSW & Northeast & Northwest \\
\hline Wind & 30.9 & 4.5 & 11.0 & 7.1 & 8.4 \\
PV & 20.4 & 8.6 & 10.9 & 0.7 & 0.2 \\
CSP & 1.4 & 1.1 & 0.3 & 0.0 & 0.0 \\
DG & 10.4 & 5.7 & 3.8 & 0.7 & 0.1 \\
Others & 34.9 & 12.4 & 9.1 & 4.1 & 9.3 \\
\hline Total (GW) & 98.1 & 32.3 & 35.2 & 12.6 & 18.0 \\
\hline Penetration & $64.4 \%$ & $61.6 \%$ & $74.0 \%$ & $67.6 \%$ & $48.3 \%$ \\
(\% of US generation dispatch) $^{6}$ & & & & & \\
\hline
\end{tabular}

\footnotetext{
${ }^{6}$ The penetration levels shown in this table were calculated considering only the U.S. portion of WECC generation.
} 
As described above, the evolution from the Light Spring Base case to the Light Spring Hi-Mix case and then to the Light Spring Extreme case displaced thermal generation, especially in the Northeast and Desert Southwest regions.

The following figure illustrates the changes in dispatch with increasing wind and solar production. Figure 9 shows the regional dispatches by generation type for the three Light Spring cases for the two regions in the eastern part of the interconnection. Because the distributed PV appeared as a load modifier, it was not included. This reflects the reality facing grid operators, because the embedded distributed generation will likely remain largely outside of operator controls, and absent monitoring and estimation systems may be nearly invisible to operators. The figure shows the dramatic reduction in coal dispatch (dark blue bar) as the wind and solar generation increased from the Base case to the Hi-Mix case, then a further drop in the Extreme case. The "Gas+" component of the generation dispatch consists of combined-cycle plants, peaking combustion turbines, oil-fired generation, geothermal, and biomass.

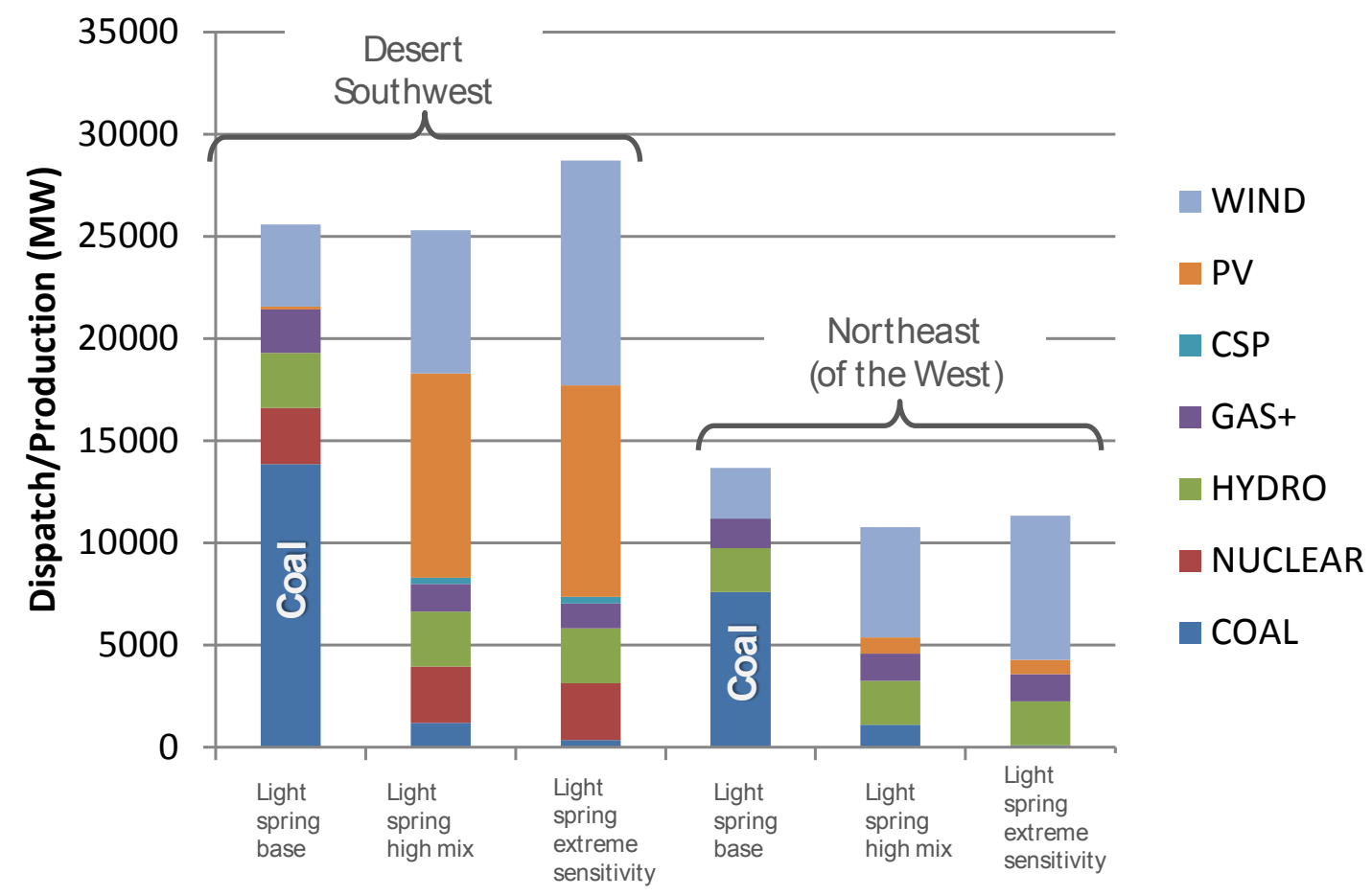

Figure 9. Dispatch details in the Desert Southwest and Northeast regions

\subsection{Details of Initial Conditions and Aeolus Fault}

Much of the analysis for this investigation centered on the Aeolus fault. Aeolus is a new 500$1230-\mathrm{kV}$ substation at the eastern terminus of the new Gateway West and South projects. Per the discussion in Section 2.1, both projects were included in the cases.

In the Extreme sensitivity case, approximately 1,800 MW were evacuated from the wind-rich region on the two new 500-kV lines. As Figure 10 shows, this power was split relatively evenly between the two paths, with approximately $100 \mathrm{MW}$ more flowing on the Gateway West project for this condition. Most of the study cases in the next section were for a three-phase (zero- 
impedance) fault at the Aeolus 500-kV line on the Gateway West line that tripped the circuit west to Anticline. The fault is modelled as clearing in four cycles $(67 \mathrm{~ms})$. The fault-clearing times used in stability studies are usually conservative approximations of actual protection system behavior. This behavior is based on the level and character of short-circuit currents, which are substantially different and less well known from inverter-based generation. Whether these assumptions are still valid in systems with predominantly inverter-based generation warrants further investigation, but is outside the scope of this study.

In the remainder of the report, results for 2 of the 20 transmission areas of the WECC power flow data sets are shown. These areas are defined by WECC and mostly align with the territories of the major transmission operators in the interconnection. Area 65 is "PACE," which is predominantly PacifiCorp's eastern territory, and it includes the future Aeolus substation. Area 73 is "WAPA RM," or the Rocky Mountain region of the Western Area Power Administration, and it includes the region to the east and north of Aeolus, including St. Onge, Lange, Lookout, and other buses in the general vicinity of Rapid City, South Dakota.

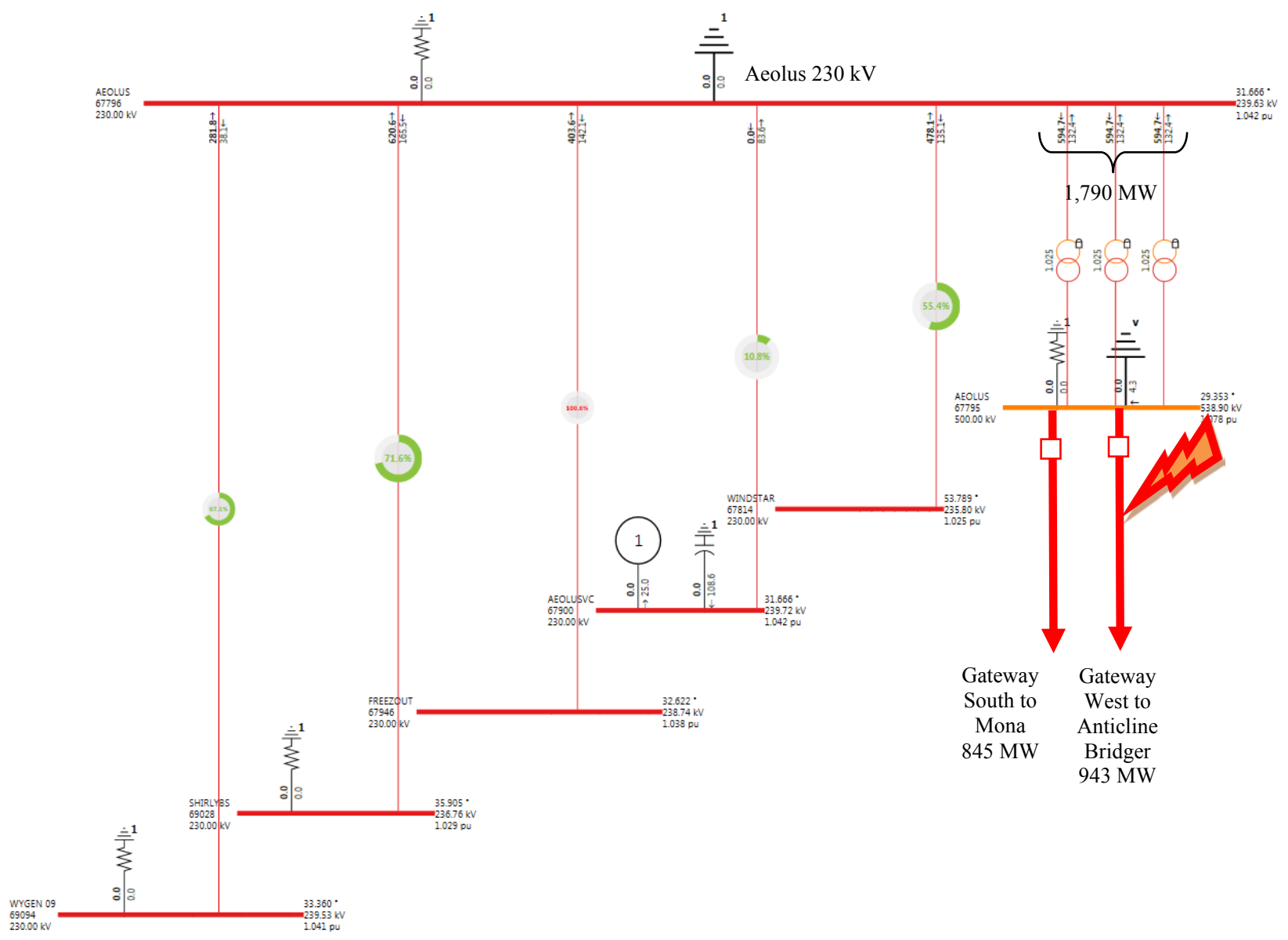

Figure 10. Flow details in the vicinity of the future Aeolus substation 


\subsection{Model Replacement and Performance Validation}

Both the Hi-Mix and Extreme cases included a significant number of new wind and solar power plants to achieve the target penetration levels. All of these "added" wind power plants were represented as manufacturer-specific Type 3, doubly-fed, asynchronous machines with voltage regulation and low-voltage ride-through in WWSIS 3. For this analysis, the wind turbine generator (WTG) models and associated controllers were replaced by the new, WECC Renewable Energy Modeling Task Force (REMTF), second-generation, generic renewable models for Type 3 hybrid network interface machines (see discussion in Section 3.7 and Figure 11 , below).
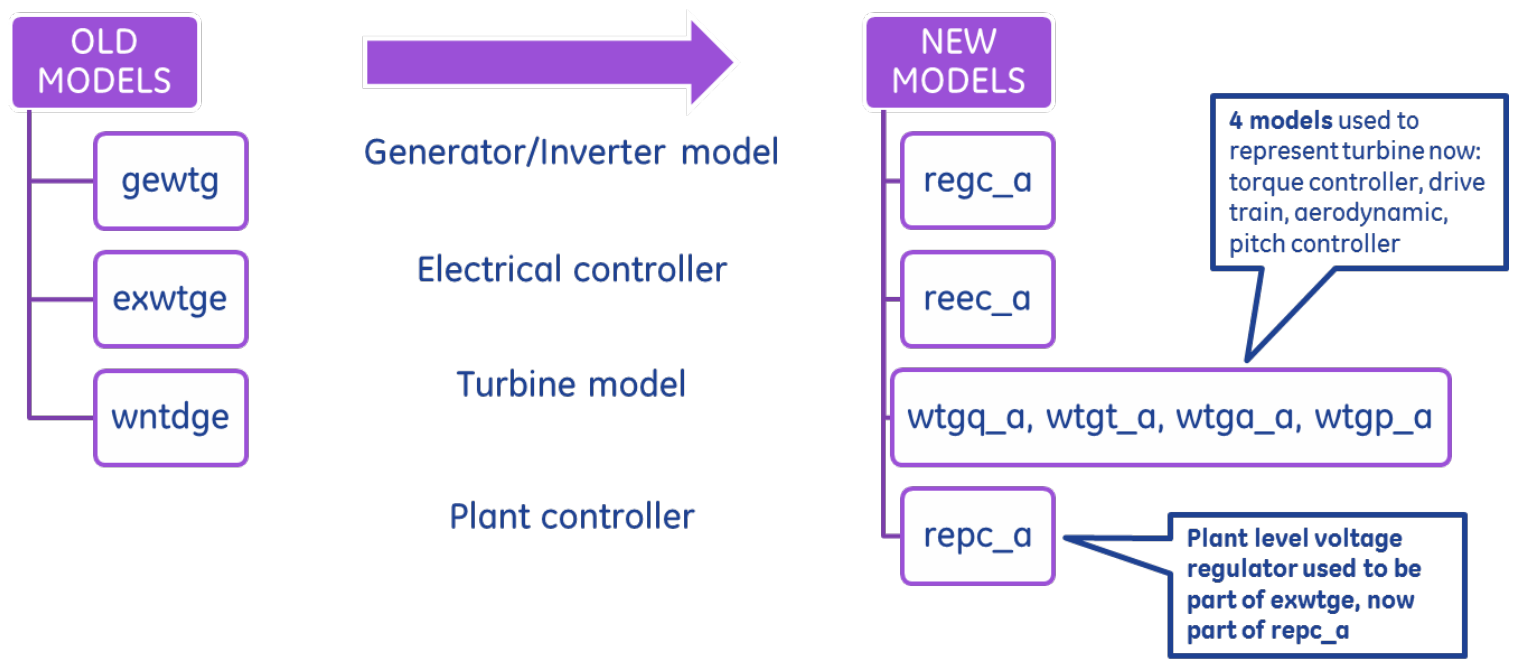

Figure 11. WTG model update

Further refinements to other dynamic models were made to correct poor initialization and other minor problems. The baseline performance investigation was started after the refined data sets gave good initializations. 


\section{System Transient Stability Performance}

\subsection{Overview}

In WWSIS-3, the initial tests of transient stability in eastern Wyoming showed system collapse during the Aeolus fault for the Extreme sensitivity case. To achieve stable operation for that event, synchronous condensers placed at Wyodak, Dave Johnson, and Laramie were used to strengthen the grid. In broad terms, it is well understood that transient stability limitations can always be mitigated by the addition of "enough" transmission.

In this investigation, more emphasis was placed on understanding the local behavior driving the stress and instability. After diving into the details of the characteristics of the system instability, the investigation turned to potential system adjustments and control features of WTGs as means to reestablish stability.

Note that this was an investigation to increase understanding about how highly stressed systems with high levels of wind and solar generation and low levels of synchronous generation behave. The industry has limited experience with large, geographically diverse grids in which wind and solar generation have displaced the majority of synchronous generation as sources of export power to load centers that are electrically distant. This was not a planning study intended to result in a specific system expansion.

\subsection{Initial Performance with Aeolus Fault}

The performance for the Hi-Mix case was observed to be quite close to that observed in WWSIS3. The changes in WTG models, transmission topology, and the conversion of most of the CSP plants to utility-scale PV had some impact on the system performance, but simulations for the refined Hi-Mix case were satisfactory.

\subsubsection{Aeolus Fault for Extreme Case}

In WWSIS-3, the voltage at the Aeolus $230-\mathrm{kV}$ bus was observed to collapse during the fault. Monitoring selected buses in the system showed that a voltage collapse was occurring to the north and east of Aeolus. System support - in the form of the conversion of three off-line coal units to online synchronous condensers - was shown to be one effective means of reinforcing the grid to achieve satisfactory performance.

In the following sequence, the grid in the entire vicinity is examined and reported. All the transmission buses in Area 65 (PACE) are shown in Figure 12. Note that this case had a short timescale for stability: the plot was terminated at the time the fault cleared (four cycles, or $67 \mathrm{~ms}$, after inception at $0.2 \mathrm{~s}$ ).

These plots do not lend themselves to inspection of individual buses, but they provide an interesting window into the overall behavior of that part of the system. Area 65 (PACE) includes the Aeolus 500-kV bus, where the fault was applied, and other transmission buses in the immediate vicinity of the electric grid. The bus that dropped to zero was obviously the faulted bus, and some other buses nearby exhibited the U-shape characteristic of buses very near a short circuit. But other buses that were relatively healthier immediately after the inception of the fault also collapsed rapidly. The most violently collapsing voltage was on a trajectory to zero at approximately $50 \mathrm{~ms}$ into the fault. In fact, it would have crossed zero, but the step size of the 
simulation $(1 / 4$ cycle) was not fine enough to show it passing through. The implications of this zero voltage during the fault are severe, and they become more apparent in Figure 13 and Figure 14.

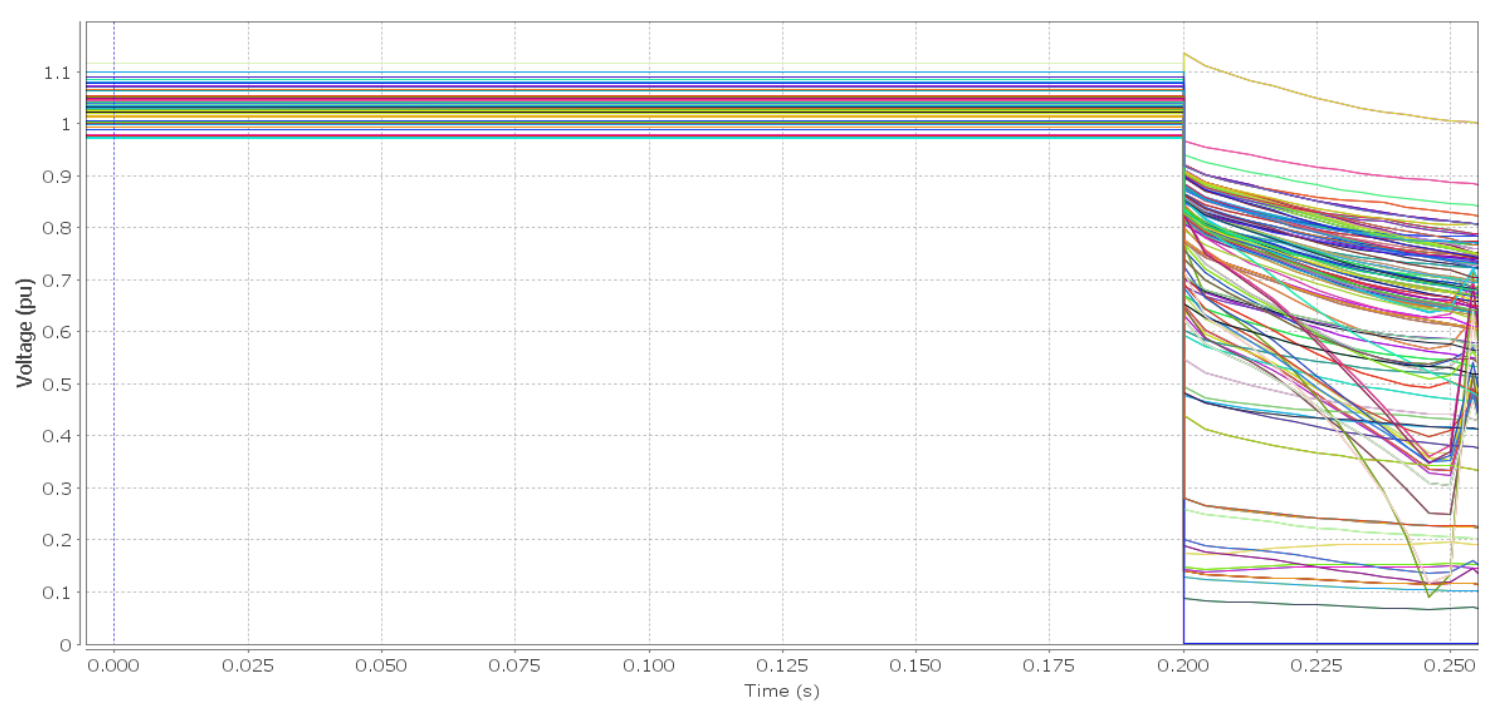

Figure 12. Transmission voltages in Area 65 (PACE)

In Figure 13, a few of the buses in Area 73 (WAPA RM) exhibited the behavior typical of a bus exposed to a deep fault some distance away. Again, these figures show the characteristic $U$ shape. These buses, even though they are identified as Area 73 (WAPA RM), are physically deep in the Area 65 (PACE) system. They reside more or less to the west of the faulted bus. But the rest of the buses in Area 73 (WAPA RM) showed the dynamic collapse observed in some of the Area 65 (PACE) buses. This is evidence of a system separation. The buses that had the worst voltage trajectories were expected to be along the separation interface.

By inspecting the bus angles, as shown in Figure 14, it is possible to determine the side of the separation for each bus. The angle plots in the figure are a little confusing to read at first. The angle reported is relative to the system reference in the San Francisco area. Mathematically, the angle was constrained between \pm 180 degrees, so the buses that experienced the most violent swings, such as the orange/brown trace for the Lookout substation, reached +180 and snapped to -180 as the separation continued.

Figure 15 shows the seam along which the subsystem in the vicinity of Rapid City tore away from the rest of the grid. In the high renewables topology (see Section 2 for more details), several very large, new wind power plants were added in this region. The generation from those wind power plants completely dominated the dynamic behavior of this corner of the system, especially for this extreme case.

A total of 8,610 MW capacity of wind and $721 \mathrm{MW}$ capacity of utility-scale solar PV was in Area 73 (WAPA RM) in the high renewables topology. The total power production coming from that fleet was 6,287 MW and $439 \mathrm{MW}$, respectively, in the Extreme case. That is almost 3,000 MW more than the production in the Hi-Mix Light Spring case. The load in Area 73 (WAPA $\mathrm{RM}$ ) was approximately $3,800 \mathrm{MW}$ for this condition, so there was a substantial export. 


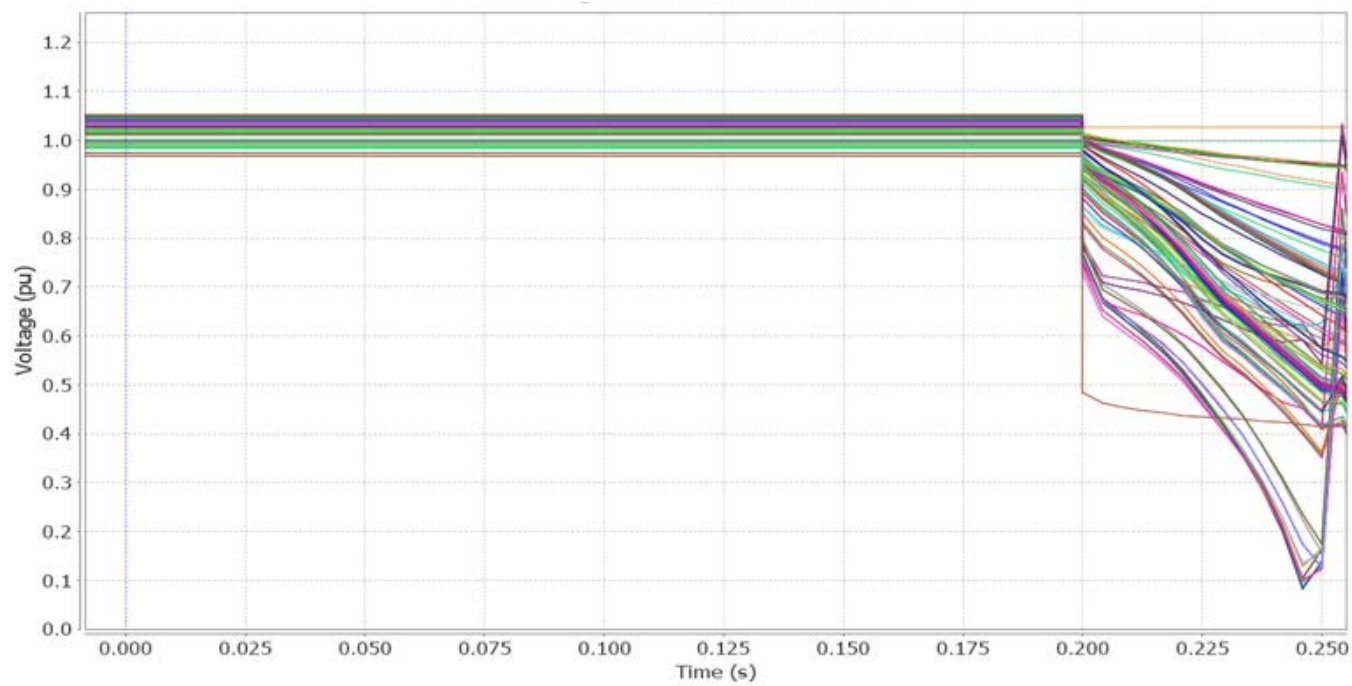

Figure 13. Transmission voltages in Area 73 (WAPA RM)

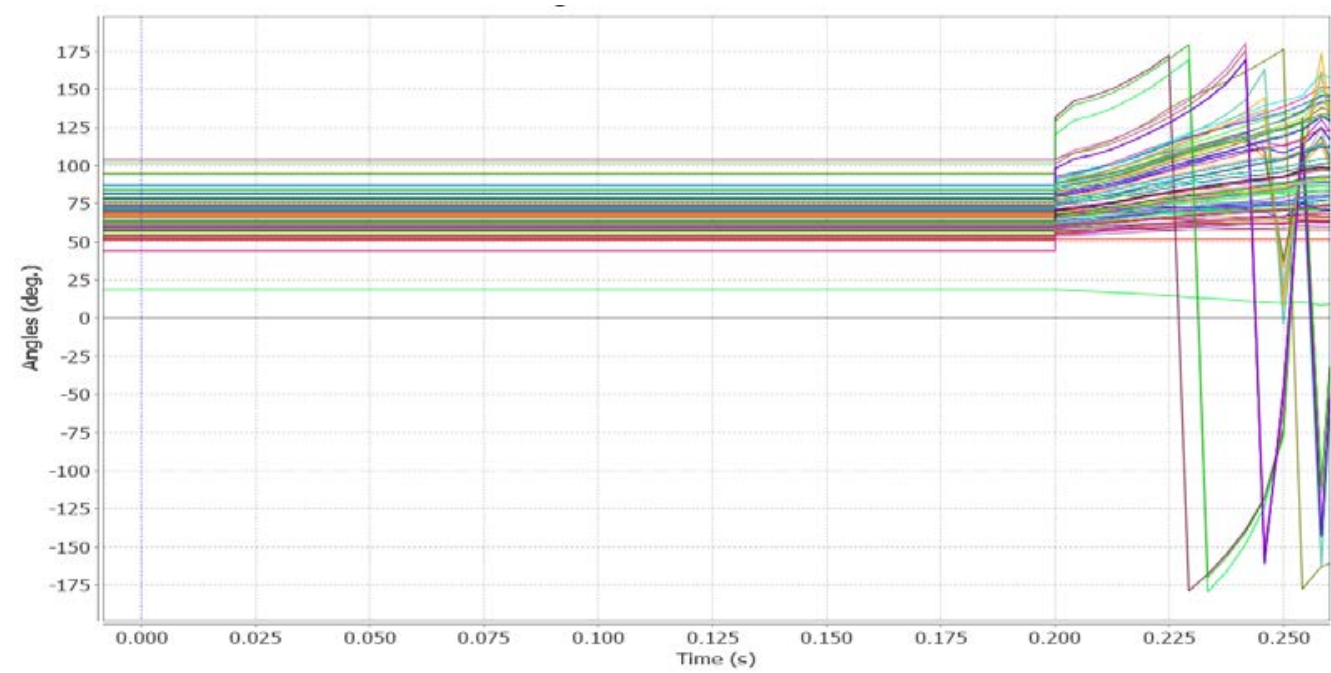

Figure 14. Transmission bus angles in Area 73 (WAPA RM) 


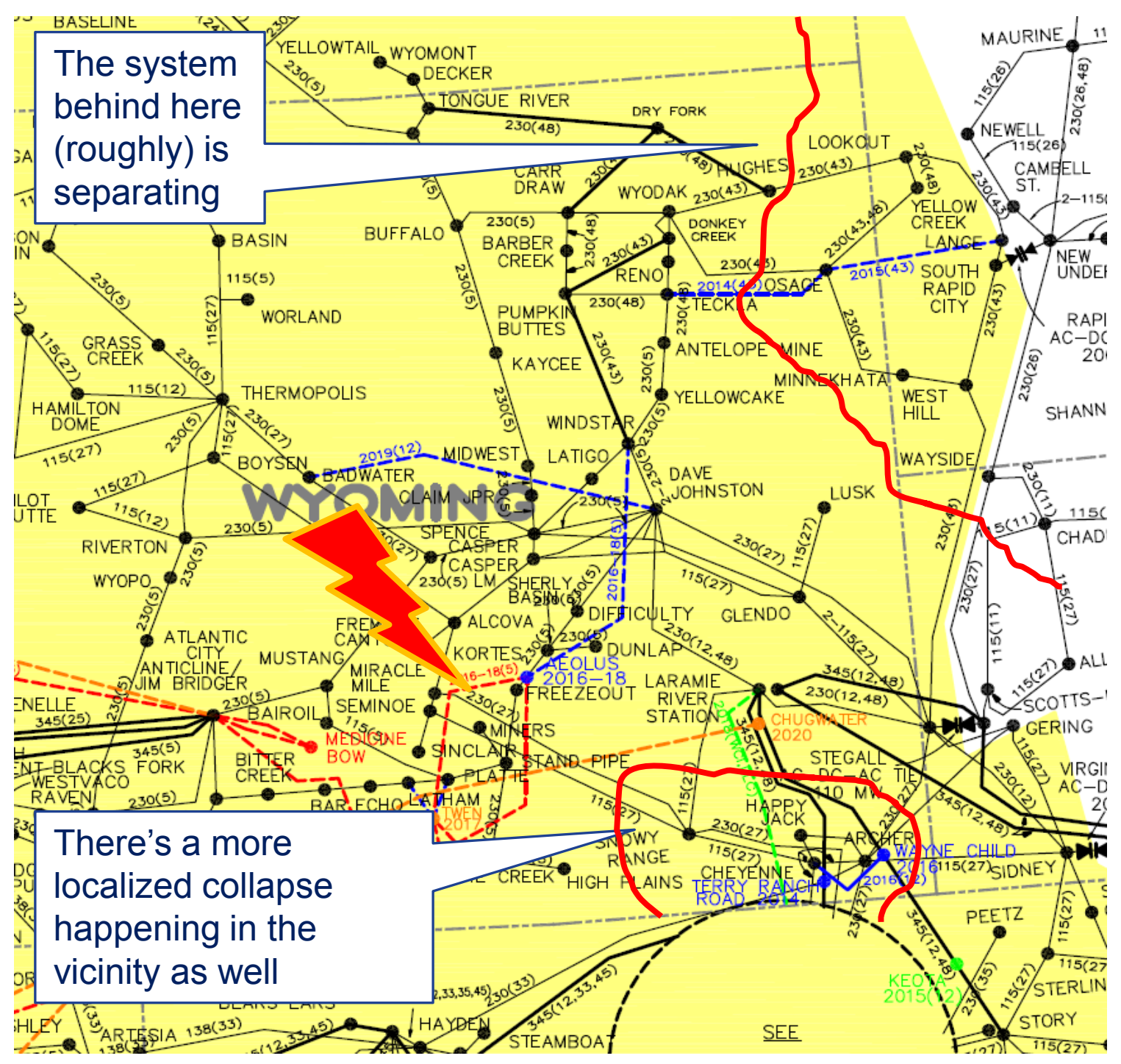

Figure 15. System separation from the Aeolus fault

The behavior of the wind power plants in Area 73 (WAPA RM) is shown in the next sequence of plots. Figure 16 plots the active power from all the plants. Because the dynamics of the WTG make its fast interaction with the grid essentially that of a current source, the power dropped instantly as the fault was applied. During the fault, the active power generally declined, becoming or crossing zero at the units in which the voltage completely collapsed.

Because the active current is a state variable, some understanding can be gained by examining its behavior. Figure 17 shows the active current (per unitized on the plant MVA base) for all the plants. The initial behavior fell into two categories: the current increased at some plants and decreased at others. The behavior of the plants was determined by the terminal voltages of the units. The basic control objective of the generator, which is dominated by the converter current control, is to maintain active power output. Thus, when the terminal voltage dropped, the active power initially dropped and the current control attempted to increase the active current to restore the MW. This happened in some of the plants. But when the voltage dropped too far, it was 
impossible to inject active power because voltage was too low. There were two factors at play in this phenomenon, both of which were approximated in the WTG models. The fact that it is physically impossible to inject active current through a reactance to zero voltage was captured in an algebraic block in the model. This approximated a physical constraint imposed on the converter, and it was a necessary component of the model to maintain numerical stability (because the network in all stability programs is approximated with algebraic equations). The currents that dropped immediately following the fault were running into this constraint. But once the converter understood that the voltage was deeply depressed, the control understood that it should stop trying to inject as much active current as the turbine would like into the system. The control reduced the active current command. This was very fast, especially in the context of transient stability controls, but not instantaneous. The change in trajectory of some of the initially rising currents in the figure that occurred at approximately $25 \mathrm{~ms}$ into the fault is evidence of this control becoming active.

Overall, the behavior of the system is that of a network in which too much active power was being injected relative to its ability to accept the power. This fundamental behavior will be examined more below.

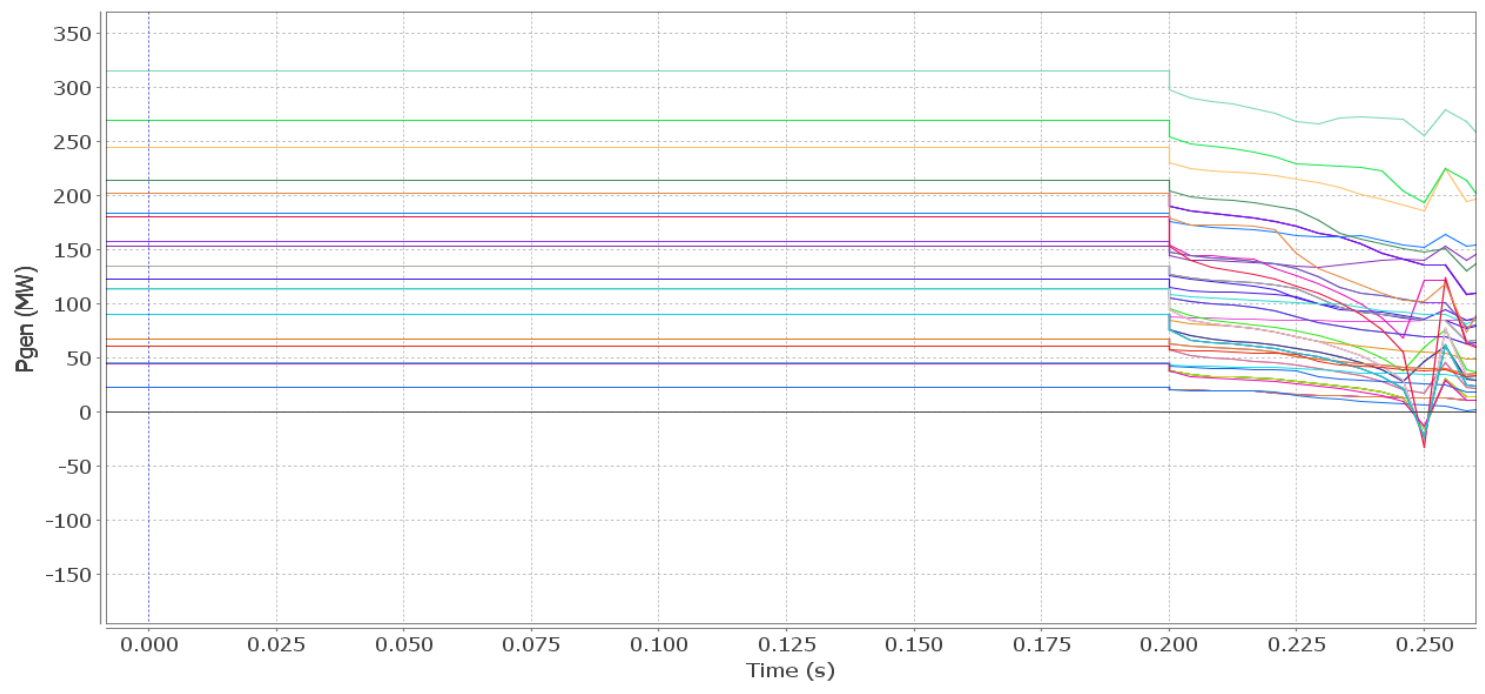

Figure 16. Active power from wind power plants in Area 73 (WAPA RM) 


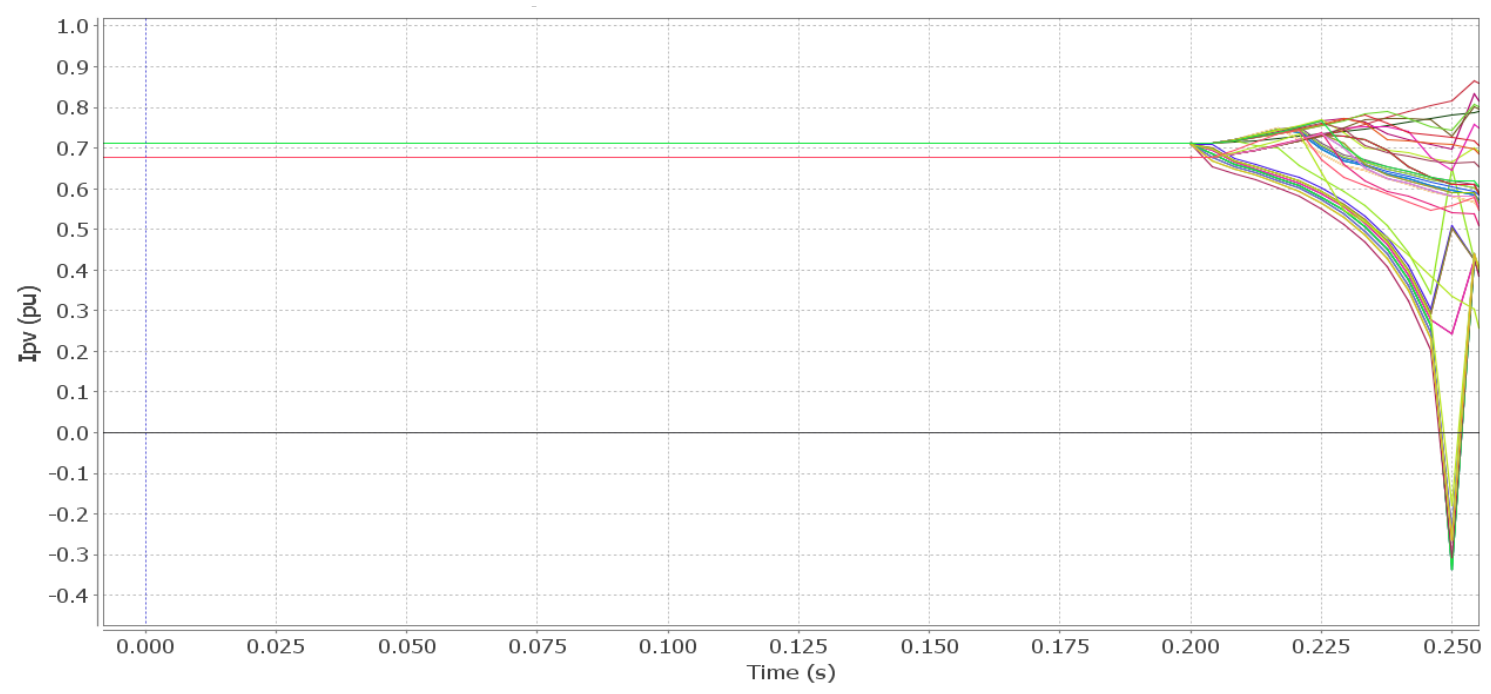

Figure 17. Active current for wind power plants in Area 73 (WAPA RM)

\subsubsection{Examination of High Stress Around St. Onge}

The separation described above led to a closer inspection of the operating conditions in the part of Area 73 that separated. One wind power plant of particular interest was St. Onge. It had a very large, 1,200-MW rating, and in the extreme case it produced $832 \mathrm{MW}$. It is also on the edge of the system, close to the east-west asynchronous interface between the Western and Eastern interconnections. While the build-out of the high renewables topology included the addition of hundreds of new wind power plants at nominally viable locations, the increase in wind power production at St. Onge and vicinity between the Hi-Mix and Extreme cases overloaded the line connecting St. Onge to Lange, as shown in Figure 18. A significant amount of shunt compensation was added in the general area to get satisfactory voltage profiles, but the fact remains that this is an unreasonably stressed condition. This condition might reasonably be considered a representation of an N-1 condition, for which no redispatch was invoked, or some other abnormal operating condition. The main point is that this is not a condition in which a planner should expect the system to behave well. Rather, this is an opportunity to learn more about the failure mode of an unreasonably stressed system. 


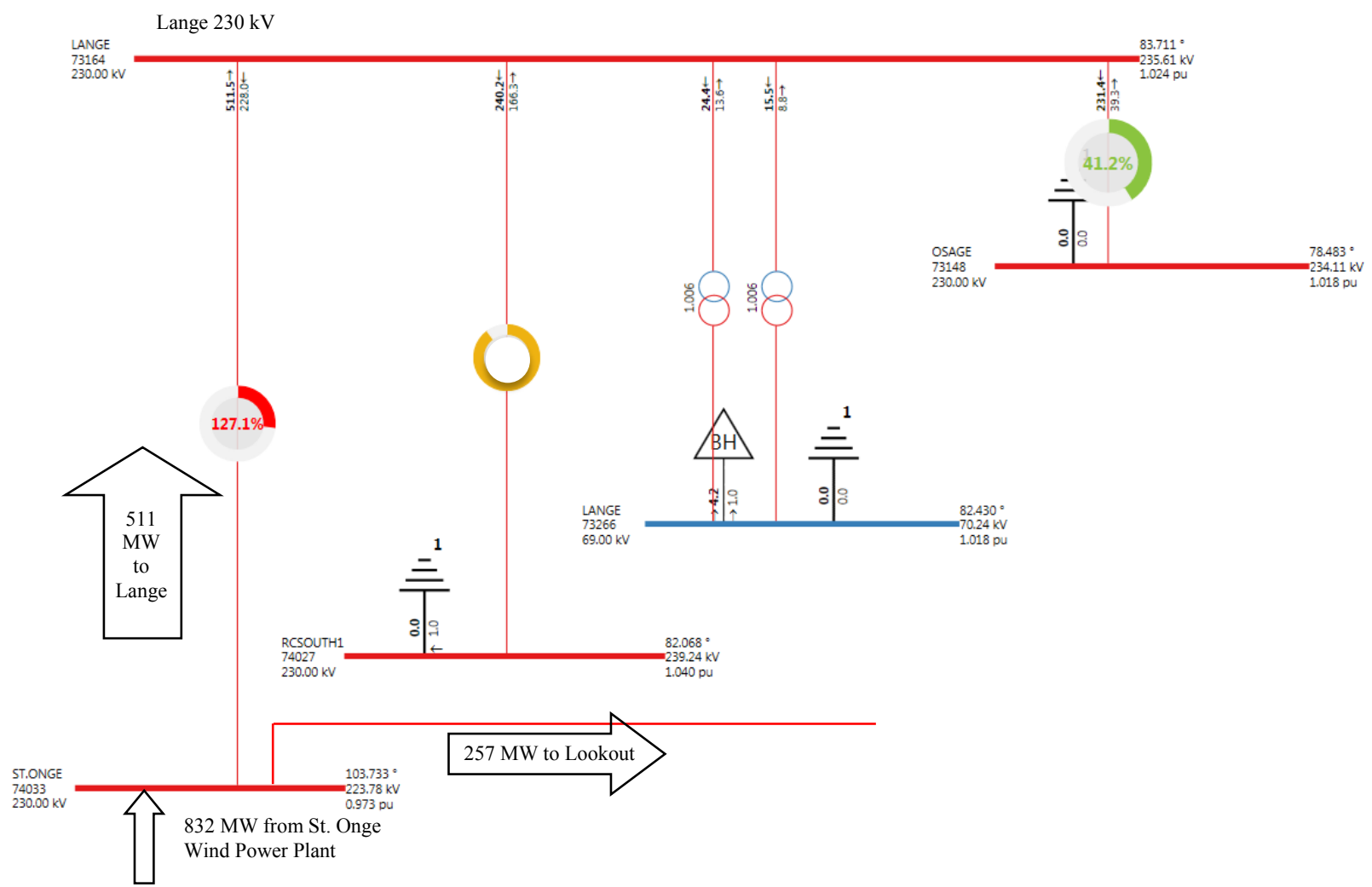

Figure 18. Overly stressed conditions near St. Onge

\subsubsection{Low-Voltage Power Limit During Fault}

In the description of the system separation above, the concept of the control deliberately suppressing the active power during deep voltage depressions was introduced. This function is generically referred to as a low-voltage power limit (LVPL). The new WECC second-generation wind model included such a function. The following observations could be made thus far:

- The mode of failure was primarily fast voltage collapse.

- The inability of the system to maintain angles synchronized appeared to be a combination of the wind turbines trying to push active power output and their inability to support voltages - especially at nodes not in the immediate proximity to the wind power plants.

- A potential mechanism to save the system is to reduce active power generated by the WTG. This action needs to occur during the fault, therefore this control needs to be very fast to be able to save the system

- Aggressive LVPL settings were shown to be effective in helping the system survive the fault in previous work.

The relevant parts of the generic WTG model are shown in Figure 19. The figure is from the WECC REMTF document of the second-generation, generic models. The two functions discussed above are noted: the algebraic limiter, which is a proxy for the physical limitations of 
the converter and for numerical stability; and the LVPL, which is representative of actual controls available from some manufacturers.

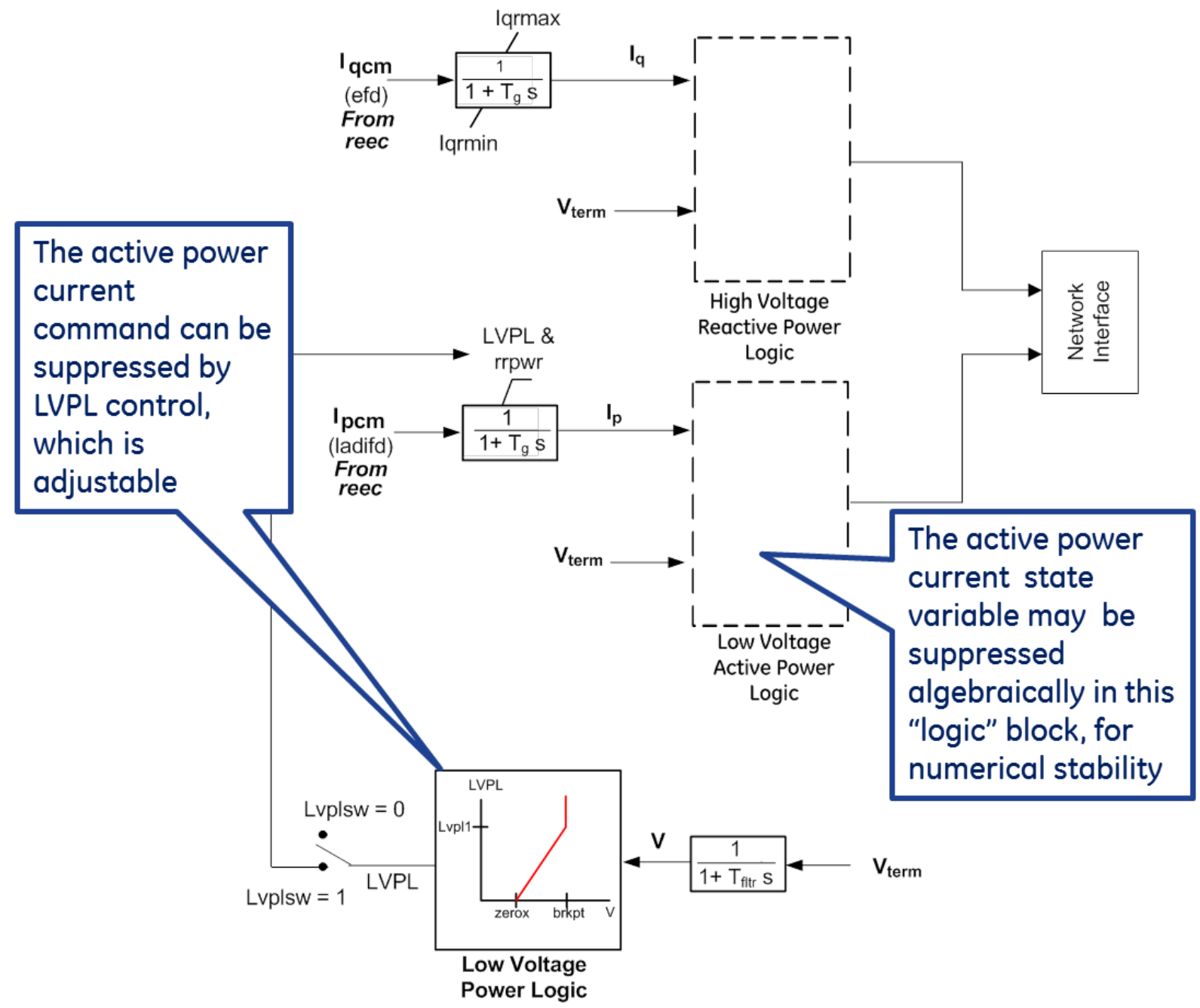

Figure 19. Model of fast control (LVPL) for power reduction during a fault

To test the possibility that rapidly suppressing the active current during the fault could save the system from separation, a test case was run. In this case, the LVPL was set to very aggressively suppress active current as soon as the voltage dropped below the normal range-i.e., it started to aggressively suppress current as soon as the voltage dropped below $90 \%$ at the terminal s of the wind turbines. It is physically possible to act this quickly, but there is no time for communications or remote measurements; the control must be purely local to the converter.

Figure 20 and Figure 21 show the transmission bus voltages and wind power plant output from all the plants in Area 73 during and shortly after the fault. During the first $25 \mathrm{~ms}$ after the fault's inception, the behavior was essentially the same as that shown above. At approximately $25 \mathrm{~ms}$, the control rapidly dropped the active current of the machines subject to the worst voltage depression. Note that the voltages everywhere rapidly rebounded, and the active power of the 
plants for which the active current was not suppressed also rebounded quickly. The active current suppression of the LVPL on the plants that were driving the separation and the fast voltage collapse allowed the system to get through the fault without collapsing.

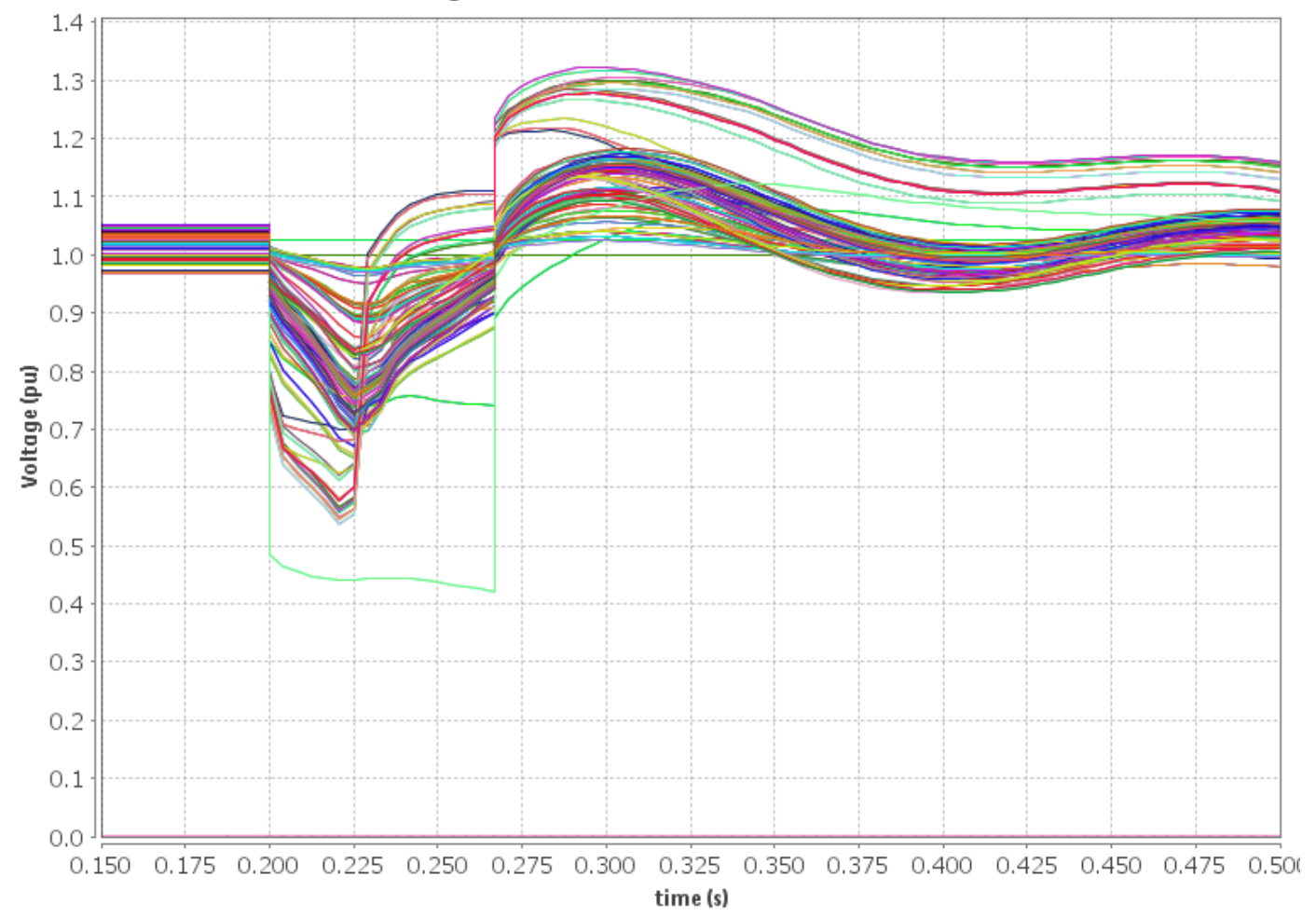

Figure 20. Bus voltages in Area 73 (WAPA RM) as impacted by LVPL

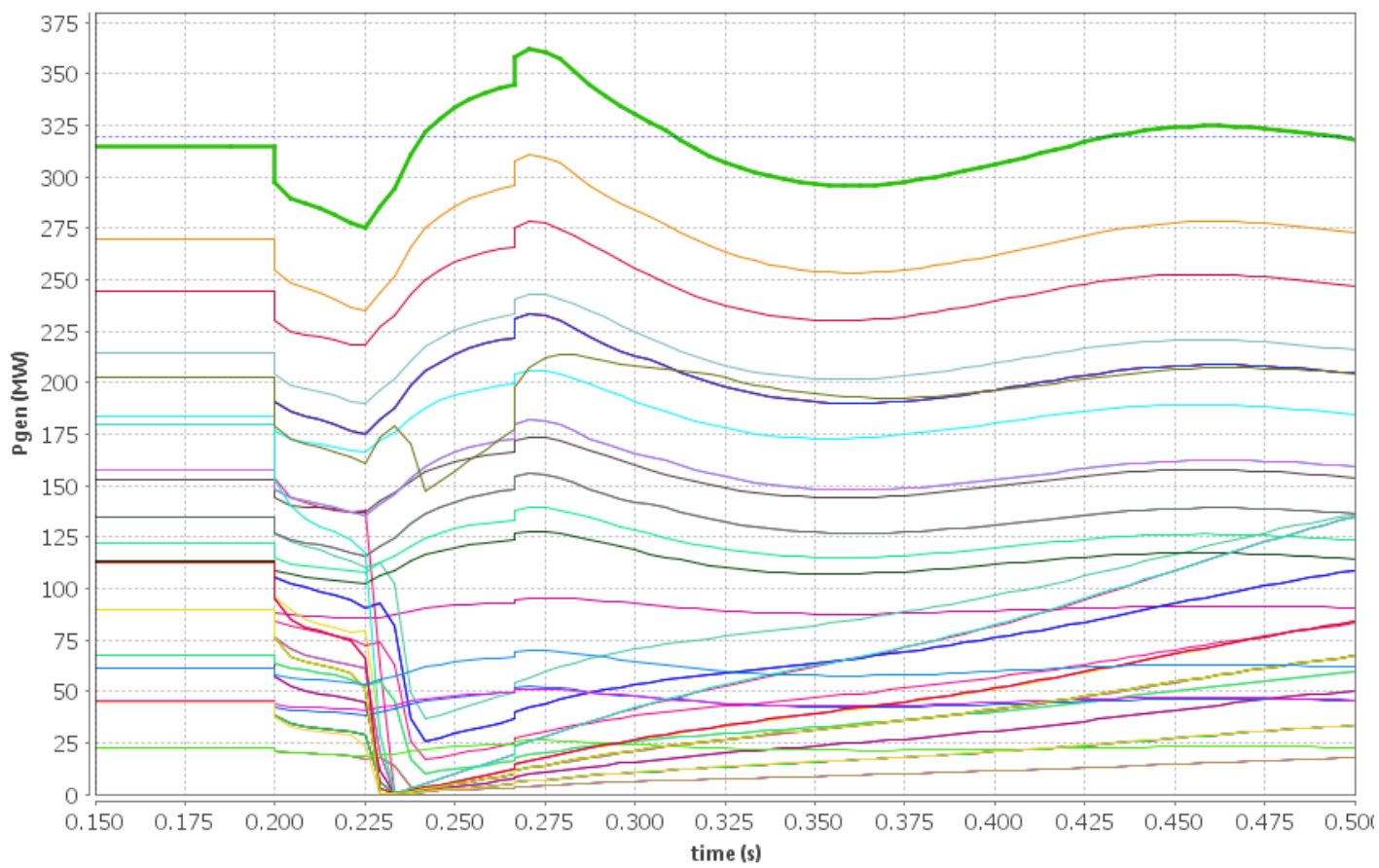

Figure 21. Wind power plant output during fault collapse arrested by LVPL 
It is encouraging that aggressive LVPL logic is able to arrest the active currents in response to low voltages and save the system from a collapse during fault. Again, note that no grid reinforcements were added to the case. However, the voltages on some buses in Figure 20 swung high upon clearing the fault. Further, the figures show only the first quarter-second after the fault cleared. Surviving the fault does not guarantee post-disturbance stability.

Once a system survives a fault by suppressing the active current, the power must be restored. The rate at which the active power recovers can have a significant impact on system stability. General Electric (GE) experience has found that, in general, when the grid is weak, it is advantageous to have the reactive current control faster than the active current recovery.

The generic model, shown again in Figure 22, included an active current ramp-rate limiter that can be adjusted to control the rate at which the active power is restored after the voltage recovers. A slow ramp rate can further allow post-fault voltage recovery before increasing active current. This is especially true in situations in which a nearby population of synchronous machines needs to decelerate rapidly following a fault to maintain synchronism. 


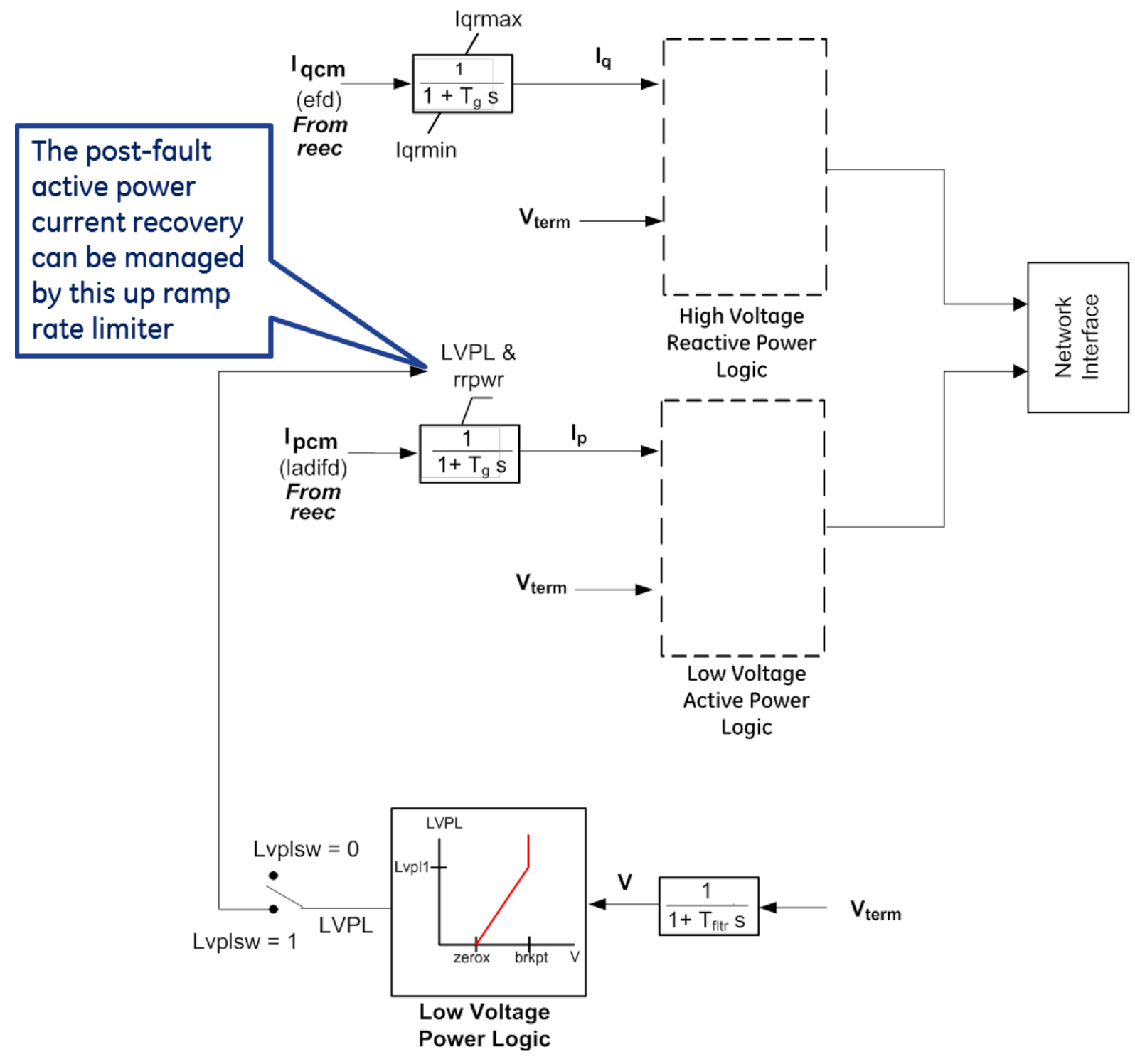

Figure 22. Active power recovery ramp limiter

However, in this particular topology, the combination of the LVPL and the controlled active power recovery created a problem.

\subsubsection{LVPL-Induced Cyclic Instability}

As noted above, immediately following the fault clearing the transmission voltages swung high, resulting in a dynamic overvoltage that may be unacceptable - though not in violation of WECC criteria. In Figure 23, the transmission voltages are shown to go into rapid, undamped, and complex oscillations. Several cycles of oscillations occurred in the first second following the fault clearing. 


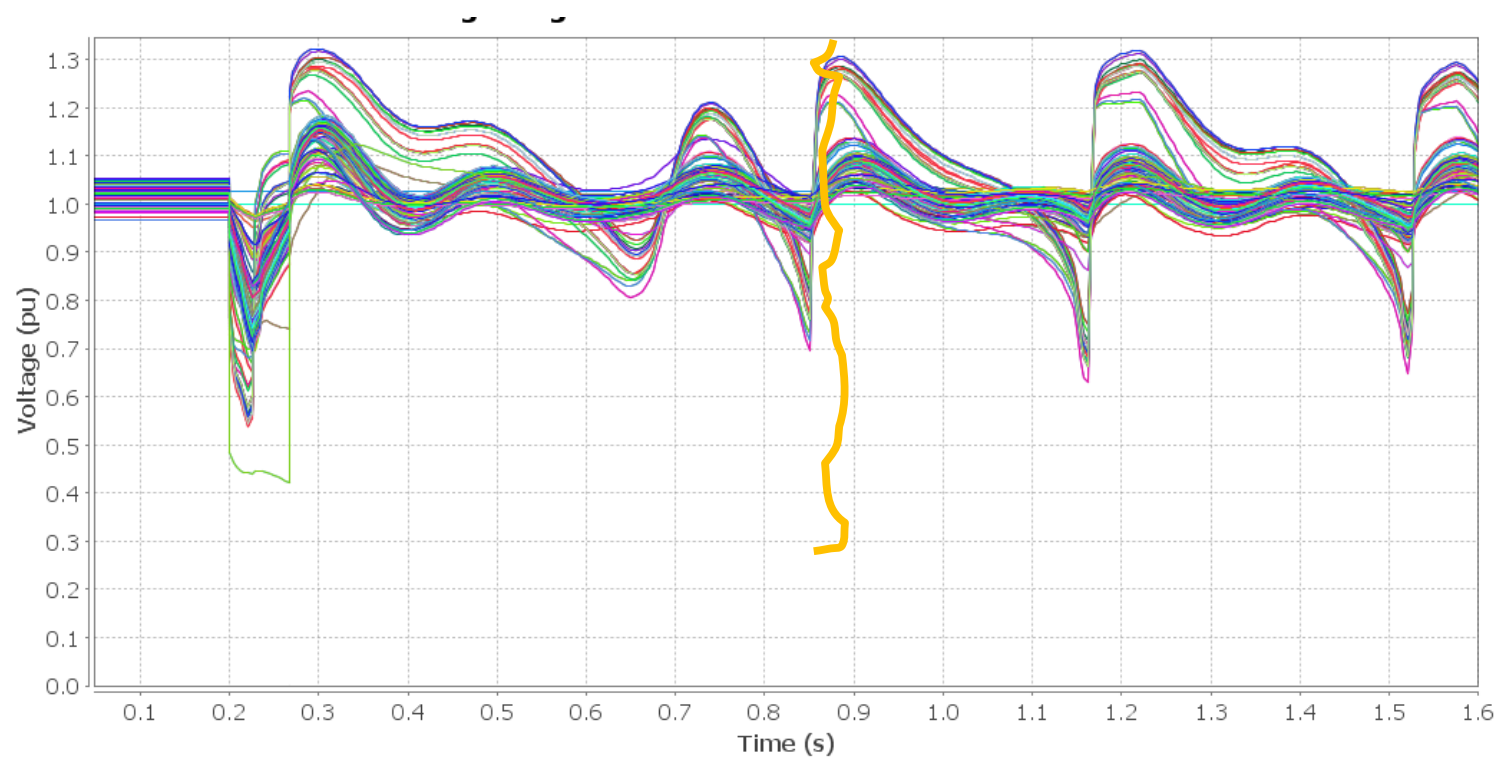

Figure 23. Post-fault oscillation-Area 73 (WAPA RM) voltages

The simulation continued through several cycles of oscillation. In practice, these violent oscillations will result in protective relay operations that will interrupt the disturbance. This type of instability has been observed in actual system operations in the United States, and it was interrupted by line protection quickly. Those protections are not modeled in this simulation, but the simulation is not very meaningful past the orange line sketched in the figure. This is, in a sense, similar to a transient stability simulation continuing to run after synchronous machines have slipped poles: the case is one of transient stability failure, and the continued calculation of the time sequence is meaningless.

The interaction between the active current and the terminal voltages on all of the WTGs in Area 73 (WAPA RM) are shown in Figure 24 and Figure 25. As noted above, the collection of wind power plants at St. Onge (approximately 1,200 MW installed capacity) overstressed the local transmission with $832 \mathrm{MW}$ of production. The behavior of one of those plants is shown in Figure 26 , which thus lends itself to a representative sample of the type of instability that affected several wind power plants in the vicinity. 


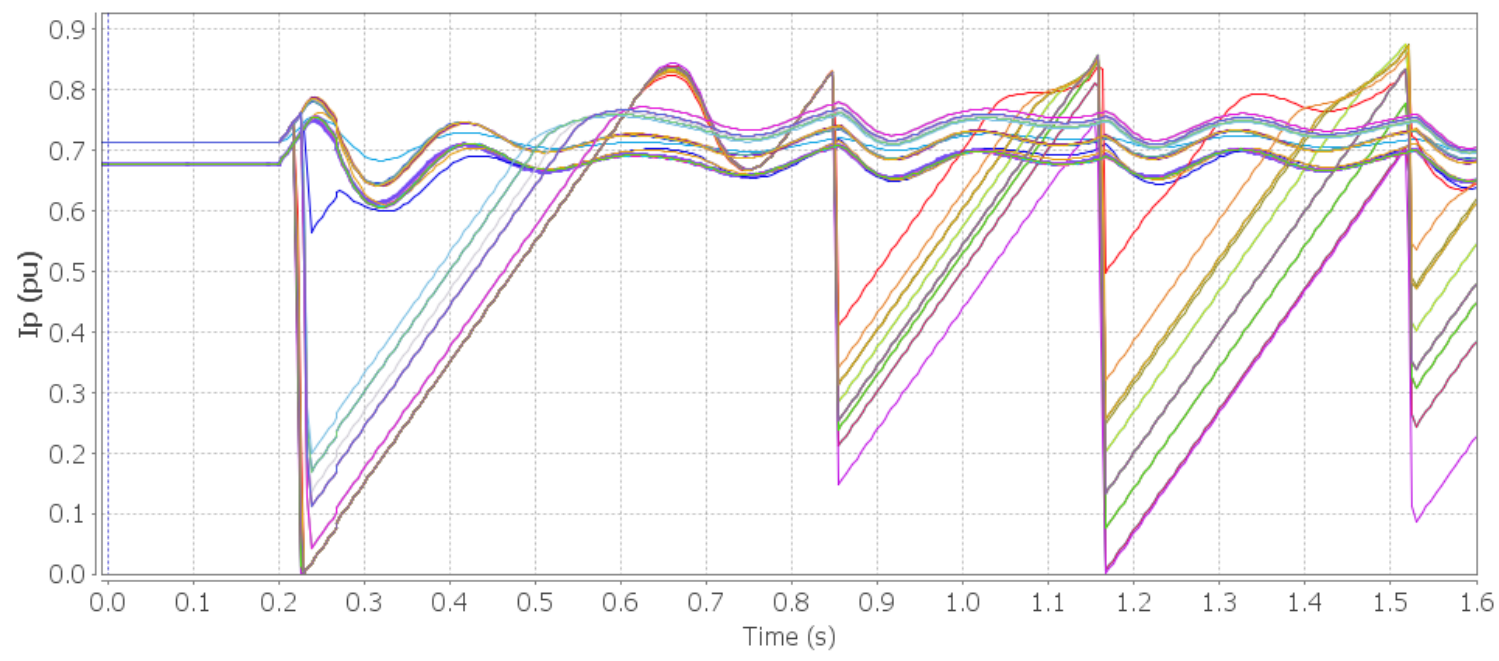

Figure 24. LVPL-induced instability in active current

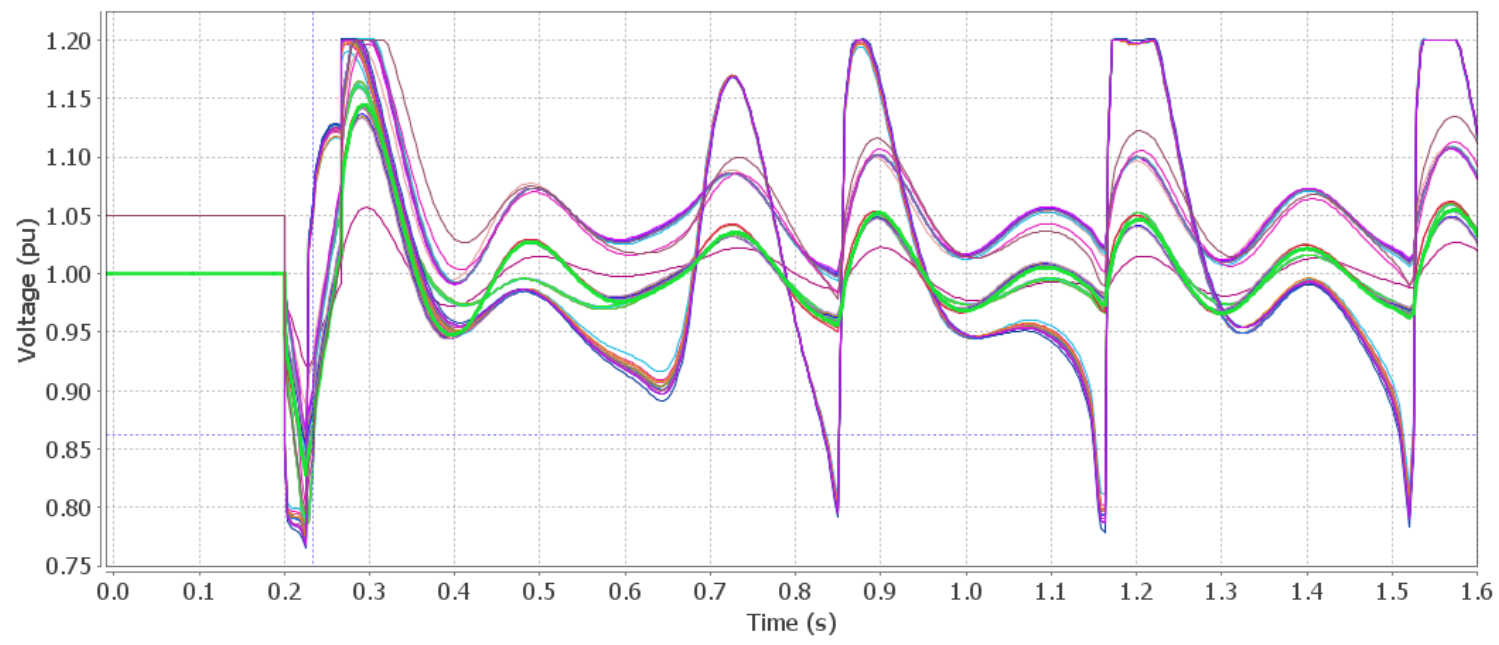

Figure 25. LVPL-induced instability observed at the WTG terminal voltages

The abrupt drop in voltage caused by the fast voltage collapse during the fault caused many wind turbine LVPLs to suppress their active current, as shown in detail below. Upon the fault clearing, the voltage recovered, allowing the active current to be restored. The rate of recovery was controlled by the ramp rate limiter (Figure 22), and it is clearly visible as the straight line segments in the figure. As the active currents neared their prefault levels, the voltage collapses triggered another suppression of the current, and a cycle repeated. 


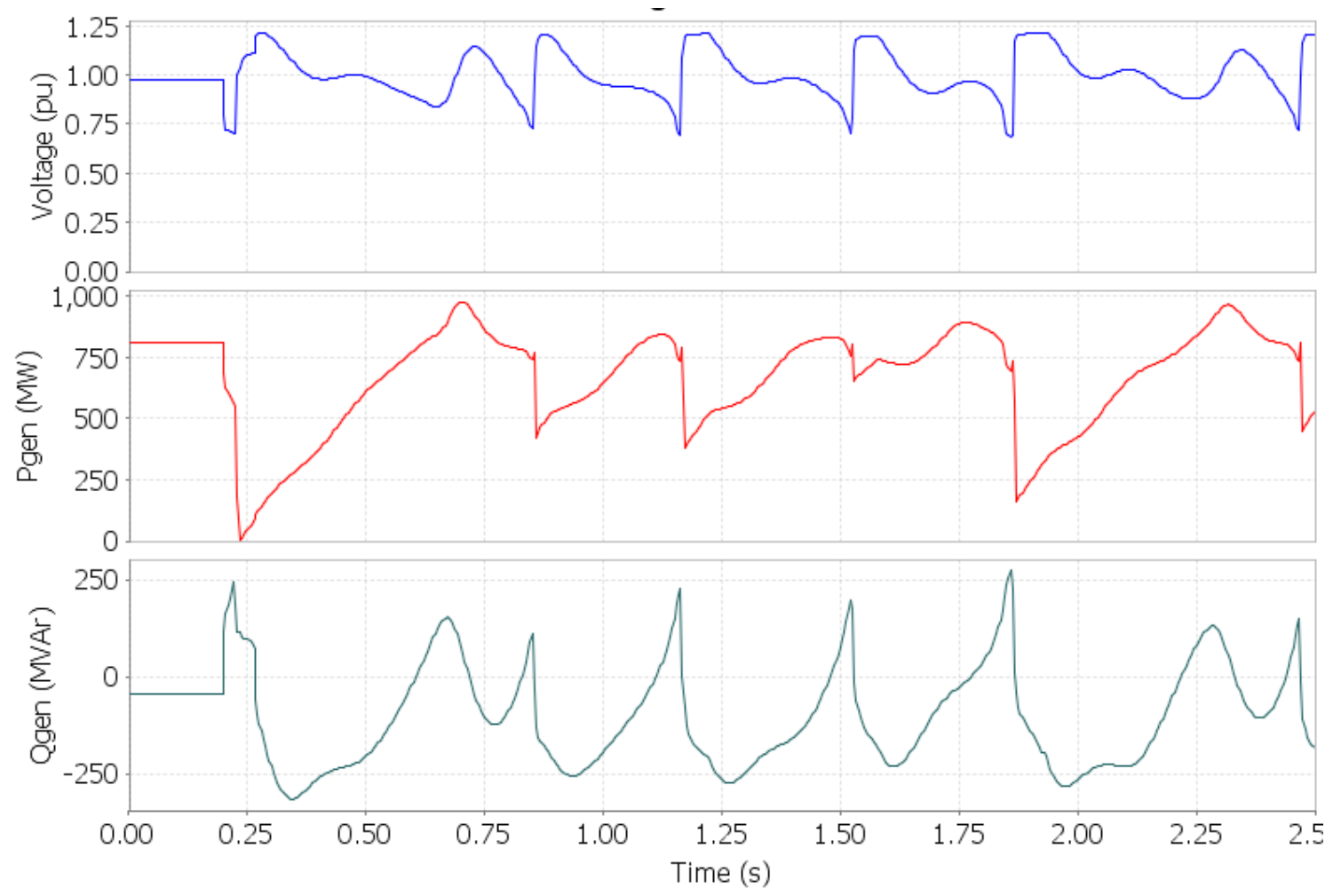

Figure 26. Cyclic instability of generation at St. Onge

\subsection{Voltage Stability Analysis}

The very rapid collapse observed during the instability indicates that the phenomenon was primarily electrical in nature: there was simply not enough time for substantial motion of the mechanical elements of the system.

In this section, the behavior is examined through the lens of traditional voltage stability analysis, starting with static analysis and then adding dynamics.

\subsubsection{Static Voltage Stability Discussion}

It is well understood that systems need to maintain some voltage stability margin from the point of maximum power transfer, i.e., the nose of the PV curve (Taylor 1994).

Figure 27 shows the PV and PQ curves generated with the power flow looking out into the system from the St. Onge WTGs. The blue traces of the curves were generated starting with the Extreme case (at the green triangle in the PV curve), and increasing the power generation of the wind power plant. The PQ curves recorded the reactive power generation necessary to maintain constant terminal voltage at the WTGs. Additional curves in red were generated with a case for which the output of St. Onge was reduced (purple square). In that case, some shunt compensation in the vicinity was changed to give a better starting profile, so the red curves and blue curves, though very similar, had slightly different boundary conditions. 
Standard power flows cannot solve beyond the point of static instability, i.e., the end of the nose. For understanding, a rough approximation of the underside of the nose curve - the statically unstable region - is sketched in green.
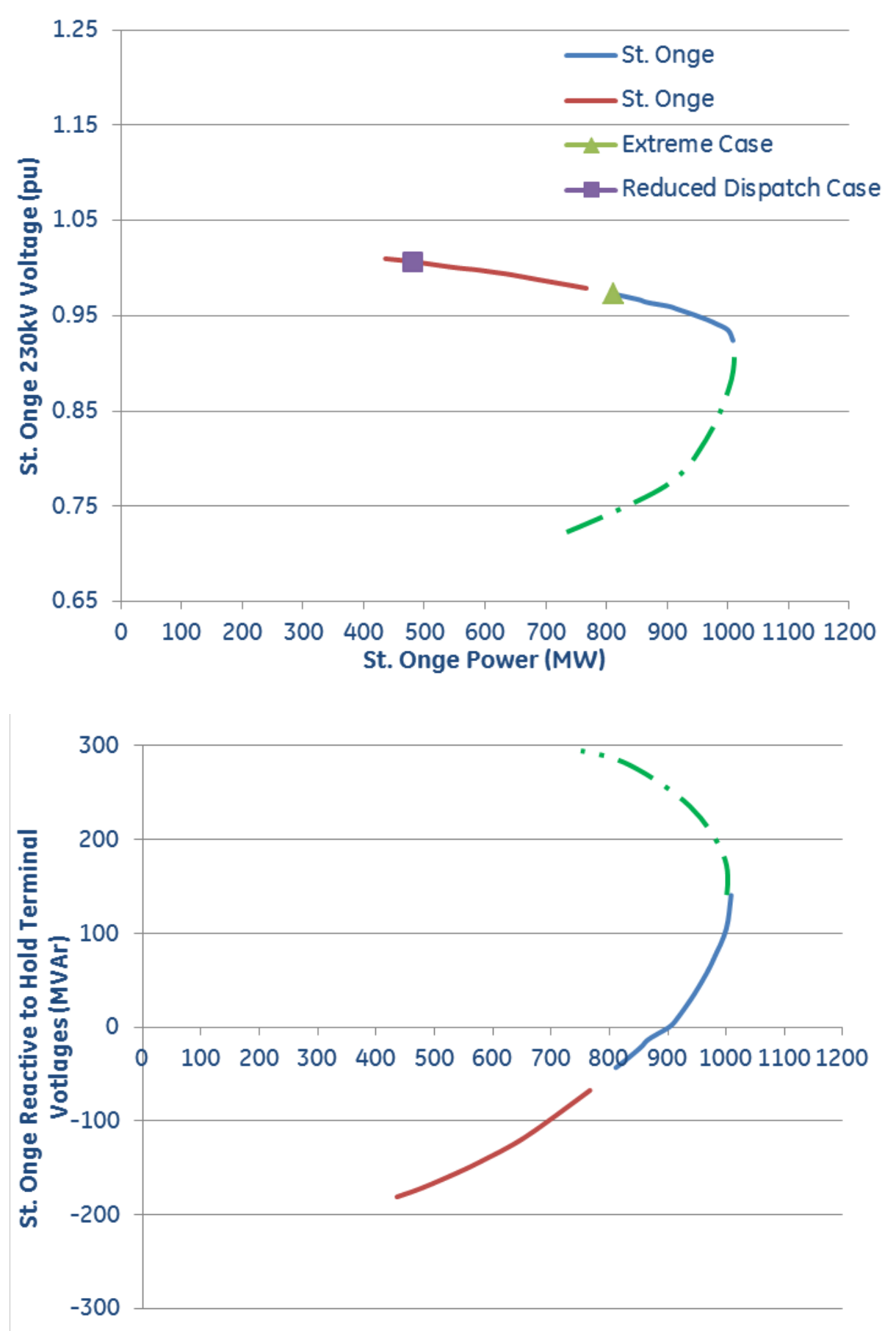

Figure 27. Load flow $P V$ and $P Q$ curves

The static boundary conditions that were enforced were not the same as those that occurred dynamically, but this exercise shows that the Extreme case, as viewed from St. Onge, was 
approximately $200 \mathrm{MW}$ from static voltage collapse when the network was intact and all available voltage control devices were meeting their local objectives or limits. The next step was to examine the problem dynamically.

\subsubsection{Dynamic PV and PQ Curves}

The time sequence of the St. Onge plant during the nonlinear oscillations is shown again in Figure 28. In this plot, terminal voltage, active power, and reactive power are shown together, to help clarify the temporal relationship. The orange box highlights the first two cycles of the instability so that they could be examined more closely.

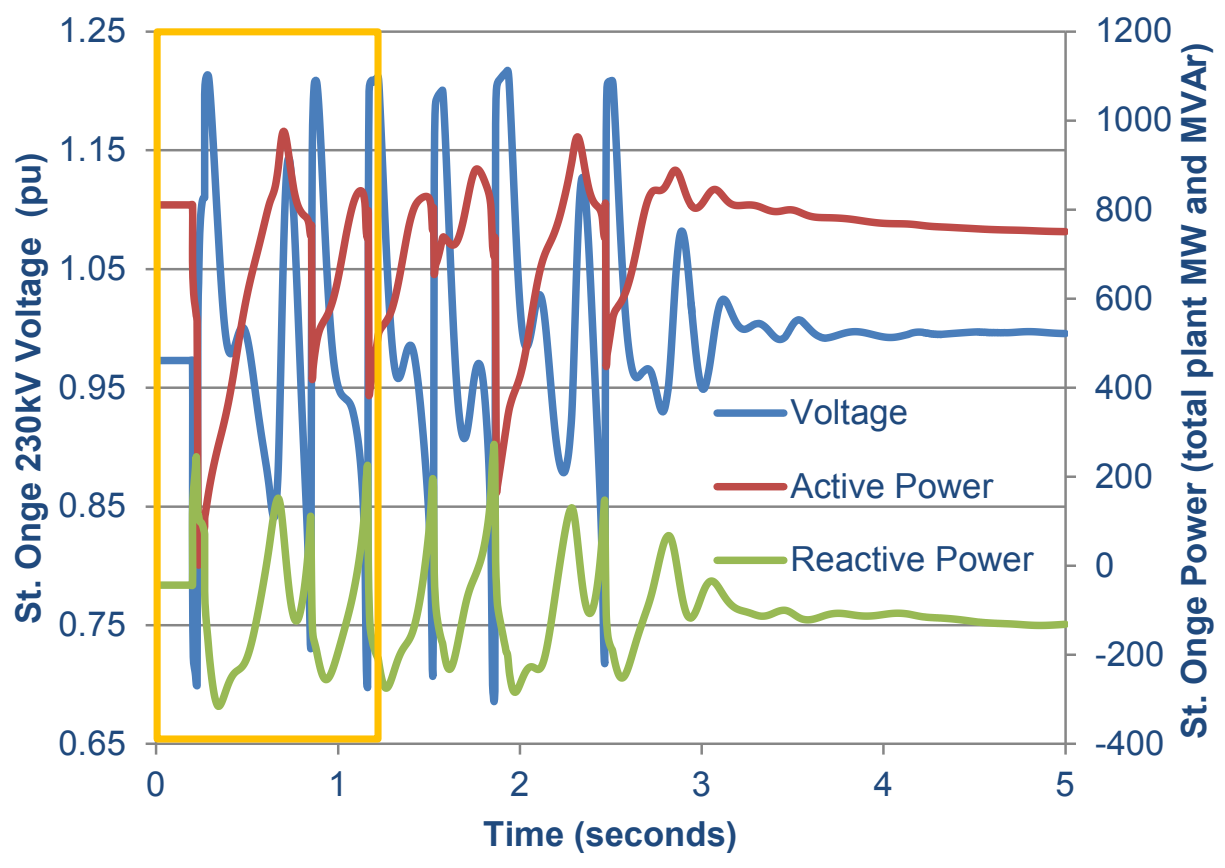

Figure 28. St. Onge wind power plant time response

Figure 29 shows the voltage and active power traces from these first cycles, with color coding:

- Blue: pre-fault

- Green: during-fault

- Purple: first unstable cycle

- Orange: second unstable cycle

The corresponding dynamic PV and QV curves are shown below in the same figure, with the same color coding. The second (orange) cycle started to reveal a recognizable "nose," but the curves were much steeper, and the power limit was in the vicinity of $800 \mathrm{MW}$ (i.e., approximately the pre-fault dispatch of the plant in the Extreme case). 


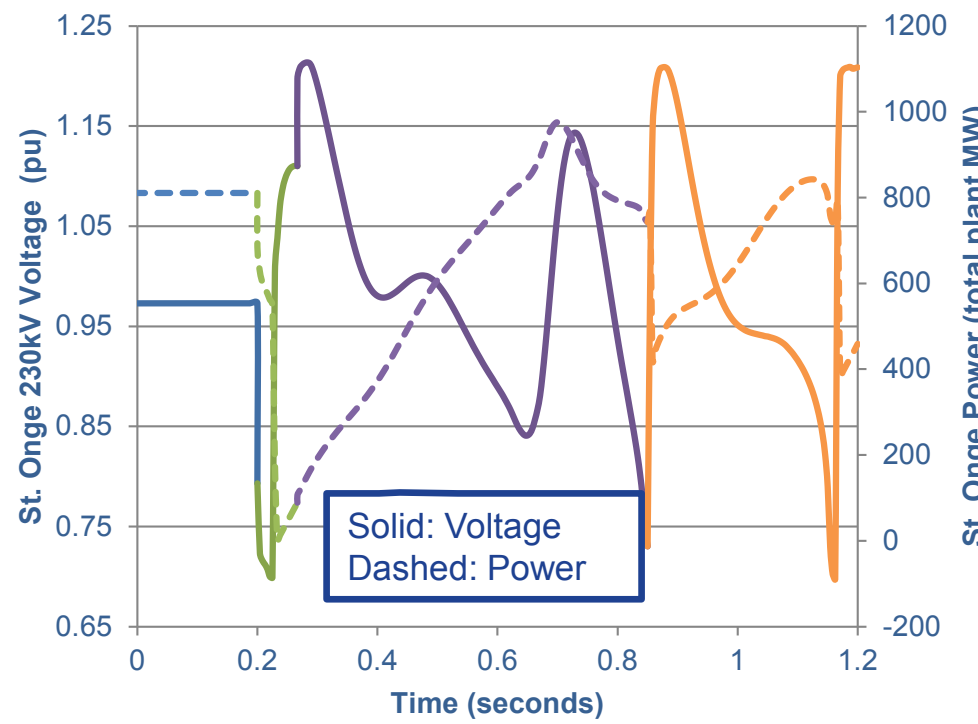

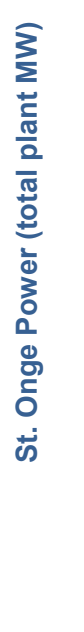
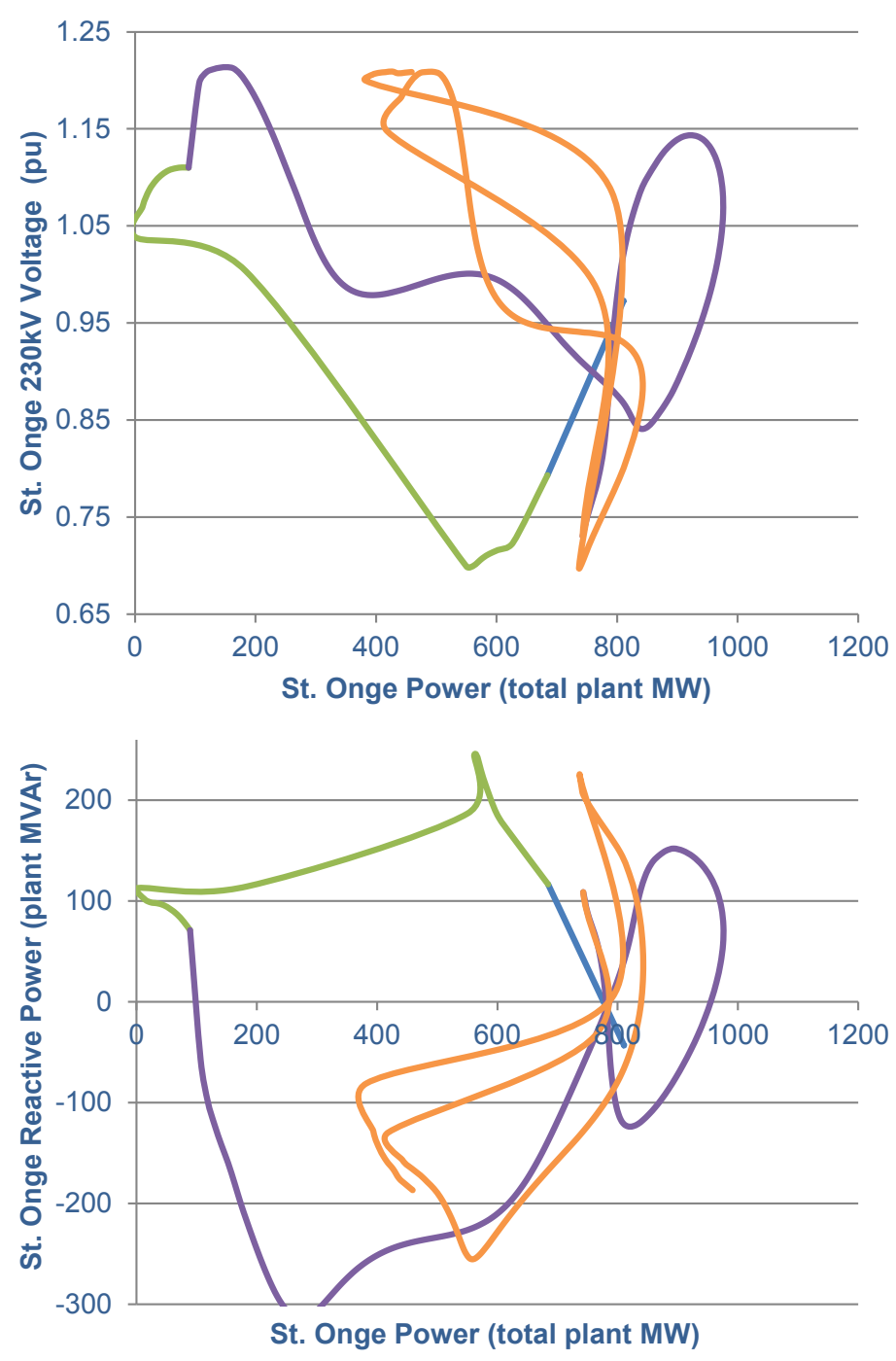

Figure 29. Dynamic PV and PQ curves at St. Onge 
This observed behavior can be understood by examining a simple voltage stability example developed by Dr. Joe Chow (Rensselaer Polytechnic Institute). Figure 30 shows the topology and a simple radial system that he constructed. In static voltage stability analysis, the source voltage (Es) is held fixed. In Chow's example, the high-voltage bus, commonly the point of interconnection was held at a scheduled voltage. In the static curves presented above, the terminal voltage (between $\mathrm{Xd}$ and $\mathrm{Xt}$ ) was held constant.

As the power injection increased, the voltage sagged, reaching the point of instability (the nose of the lower curve) at approximately $150 \%$ of the initial power. In the static curve shown above, the static collapse was at approximately $125 \%$ of the initial power.

The critical insight from this example occurs when the ability of the equivalent synchronous machine to regulate voltage is insufficiently fast to follow the change in active power. A reasonable simplification of this is holding Efd fixed. This simple example shows that when excitation is frozen (fixed Efd), the voltage stability radically changes - even though the VPQ balance of the system remains the same. The exact same VPQ point (shown by VR $=1.0$ ) is unstable the instant the voltage regulator becomes inactive for the upper curve, even though the excitation provided is the same. 


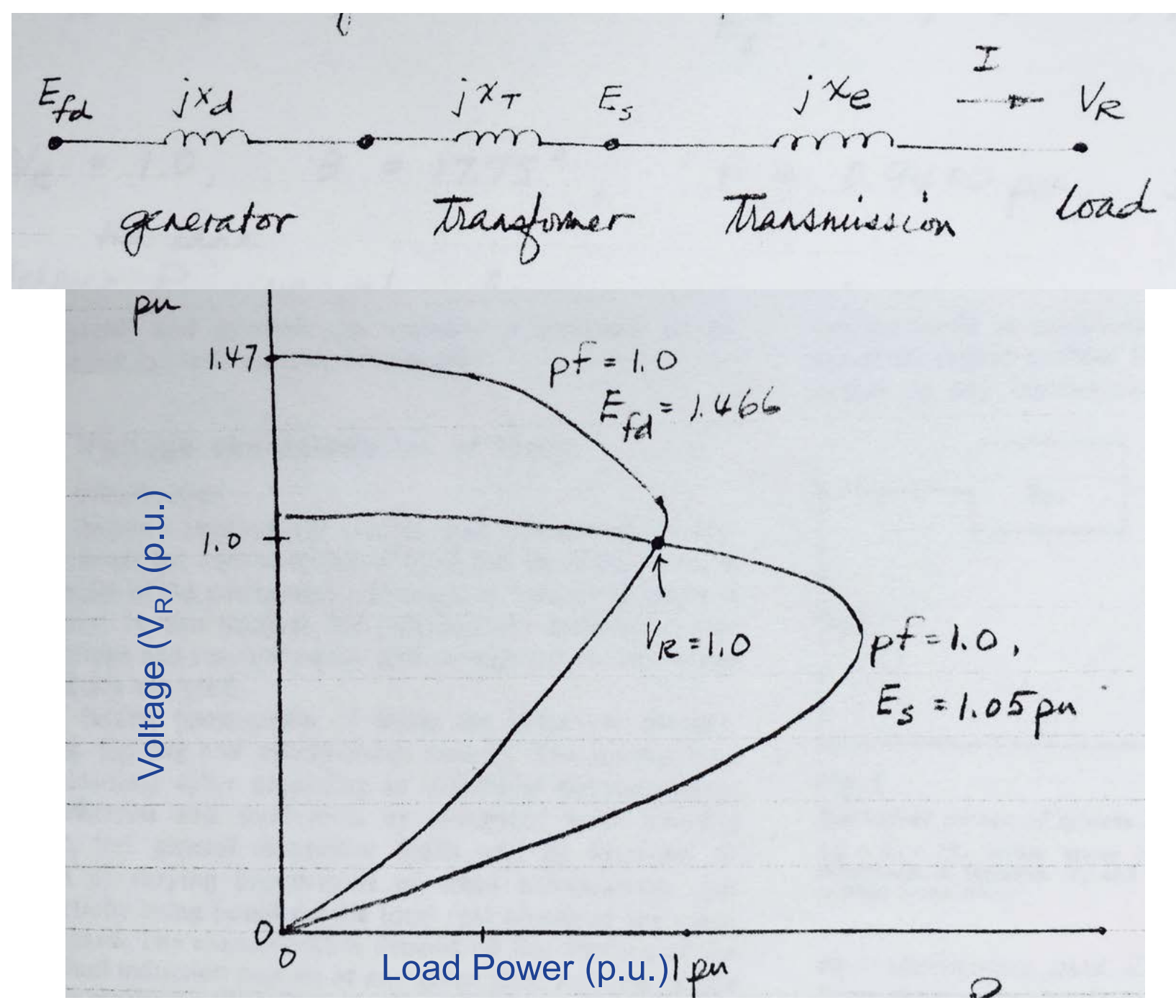

Figure 30. Dynamic voltage stability limit theory. Image from J.H. Chow 1989

Figure 31 shows a sketch of an approximation to the locus described by the unstable oscillation trajectory. The static (power flow) PV curve has also been sketched. Clearly, the topology of the Western Interconnection grid is more complex than Chow's example, but the similarities are clear. 


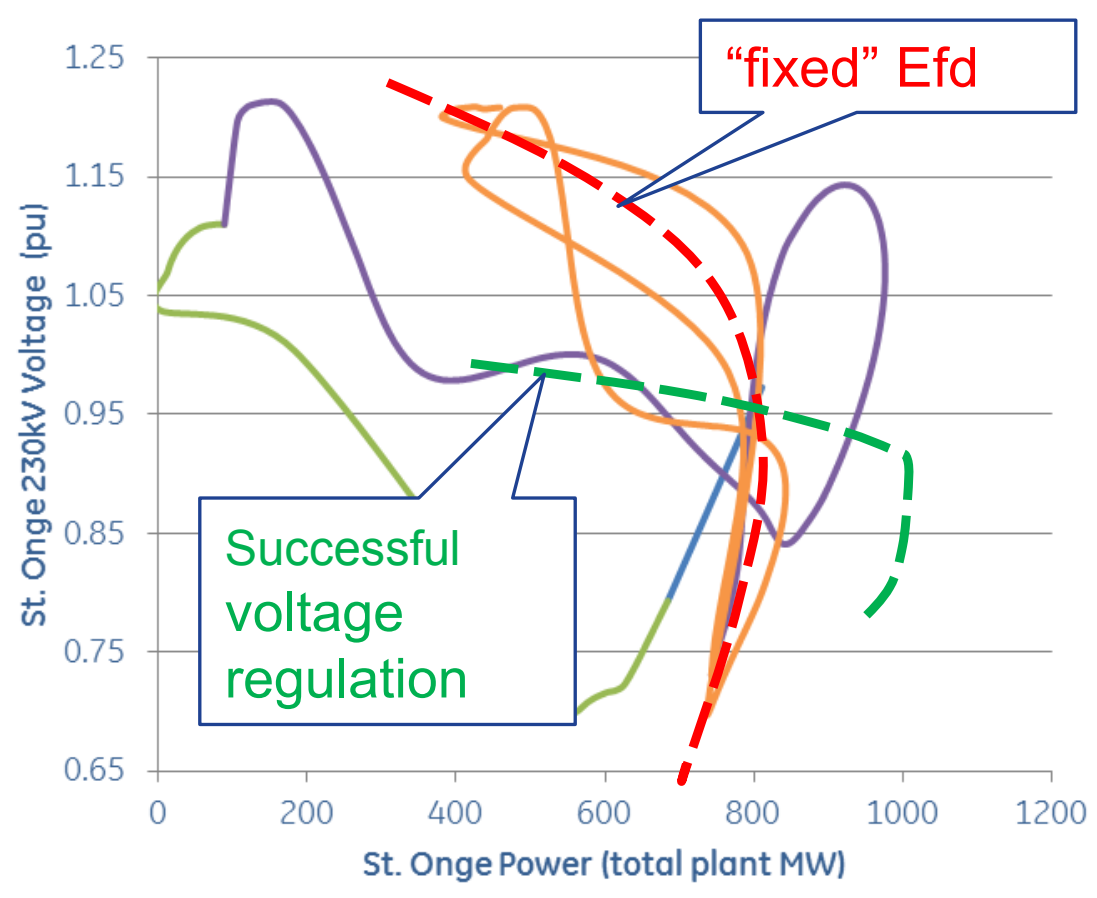

Figure 31. Dynamic voltage collapse-PV space

The genesis of the nonlinear oscillations becomes relatively clear. The time frame of the action of the LVPL is short. The second oscillation in Figure 29 occurs during approximately one-third of a second. The ability of the excitation systems on the synchronous generation in the system to respond in this time frame is limited. That means that, to a first approximation, the synchronous machines behaved in a manner much closer to that of the fixed Efd than to the ideal of successful terminal voltage regulation. The point of instability with this set of boundary conditions was at or beyond the active power level to which the wind turbines were trying to recover. As the active current rose, it pushed the system around and under the nose, collapsing the voltage and driving the LVPL to act again.

There is a temporal evolution of the system stability loci, such that as the dynamic time frame stretches out over time, the fast dynamic nose curve (the red locus in Figure 29) will evolve toward the static curve (the green locus). The static locus was stable, so by slowing the active recovery and allowing the voltage regulation of the slower synchronous machines to catch up, it is possible that the system could be made stable. Obviously, the active current is only part of the dynamic boundary conditions. The reactive behavior of the wind power plants is also critical, and better behavior of the wind power plants in that regard will also improve the system performance.

The primary observation from examining the system instability with voltage stability tools is that this behavior is not unique. The dynamic limitations of the system when overstressed and presented with a preponderance of inverter-based generation providing export are much more closely related to fast voltage collapse than to traditional electro-mechanical machine angle stability. 


\subsubsection{Nonlinear Oscillator Discussion}

Nonlinear systems are notorious for exhibiting oscillatory behavior. The type of cyclic oscillation observed in weak grids that have high penetrations of wind and aggressive LPVL differs from regular power systems oscillations in that the boundary conditions - as shown in Figure 29-are very nonlinear.

The traces in Figure 29 focus on the active power and its relationship to the grid voltage and reactive requirements. There is also a complex relationship between reactive power and voltage. Figure 32 shows the trajectory of reactive current and terminal voltage. The periodic orbit induced by the LPVL action was clearly highly nonlinear. Although this behavior is not familiar and not well cited in the literature of electric power systems, it is recognized in nonlinear system theory. This is a Van der Pol oscillator (Khalil 1996). Some methods are available for analyzing nonlinear systems with techniques other than time simulations. This is ground for future research. For instance, the use of linear analysis on small, second-order systems is a useful tool to better understand limit cycles, such as the Van Der Pol oscillator. However, for now the critical observation is that this particular oscillatory problem in a large power system is probably not going to lend itself well to linear analysis only, i.e., eigenanalysis. New approaches built on the time simulation and algebraic network analysis are likely to be needed as power systems evolve in this direction.

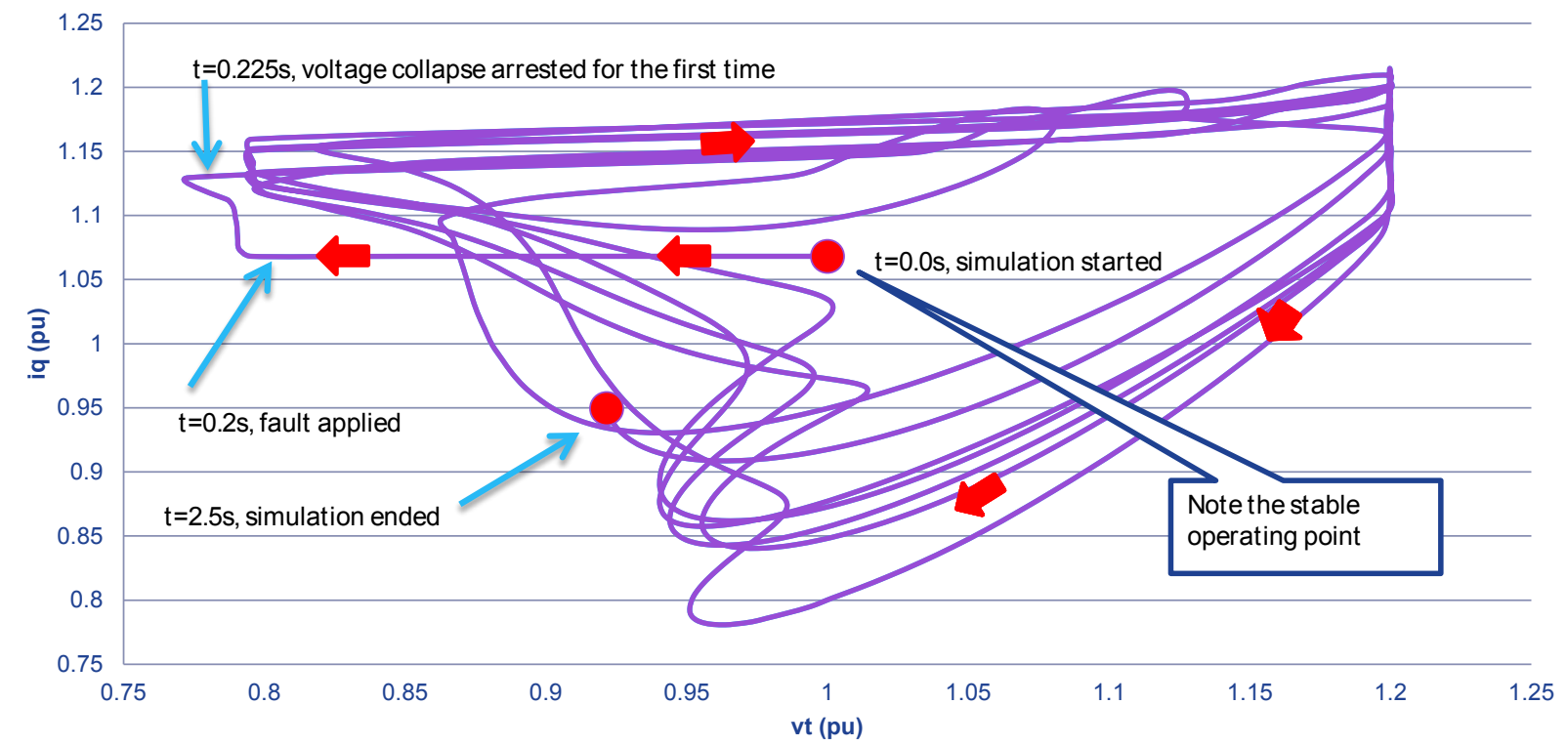

Figure 32. Phase portrait showing the limit cycle on a WTG at St. Onge

\subsection{Observations on Aeolus Fault for Extreme Cases}

The analysis presented above is for a system operating in unreasonably highly stressed conditions. This exercise was intended to help learn about its behavior; the idea was not based on the notion that this condition should work.

These cases show that aggressive LVPL settings combined with a weak grid can push the system into cyclic instability. The failed simulations were analyzed in great detail to ensure that they 
were indeed symptoms of systemic failures and not only numerical/algorithmic problems. The phenomenon has been observed upon at least one occasion in actual field operations in the United States. This seems to be characteristic of stability simulations in systems that have pockets of extremely high penetrations of inverter-based generation.

Upon close inspection, the cases reported here were well mannered up to the points at which the system failed, but the failure modes and behavior during the collapses were highly nonlinear and appeared different from the typical loss of angle-stability failures. The mode of instability shown in these cases is inverter-based generation trying to inject active power into a grid that cannot accept it. Reducing the active power rapidly when the system cannot accept the power helps considerably. For this system, the critical problem appeared to be during the fault, and therefore the mitigation (by control) needed to be very fast. Wind power plant controls are able to remedy instability to some extent. The "shape" of the oscillatory instabilities is different from what normally appears in power systems. The frequency of the oscillation is faster than the usual range for power system phenomena. This type of unstable behavior is uncommon in the context of synchronous machine transient stability. This is primarily an electrical phenomenon: the acceleration of the physical machines is not the dominant characteristic of the event.

Transmission systems can experience high voltages post fault-clearing if a substantial amount of active power is withdrawn by control such as LVPL. This is a cause for concern, and it needs to be considered in system analysis.

Protective devices are expected to trip equipment when the system enters a limit cycle. They are not modeled here, and the correct modeling of these protective devices is challenging. It is possible that these protection systems will allow the system to settle to a satisfactory postdisturbance condition. But, regardless of the modeling challenge, depending on self-tripping by protection not designed for this purpose is insufficiently robust.

\subsection{Reduced Stress Analysis}

As shown above, the wind power production from the St. Onge wind power plant exceeded the thermal rating of the evacuation circuit for the Extreme case. A normal system operation response to such an overload would be to reduce the loading on the local $230-\mathrm{kV}$ system by curtailing St. Onge. This stress reduction can be thought of as redispatch after a contingency (N1 condition).

In Figure 33, the plant has been dispatched back by approximately $300 \mathrm{MW}$, from $832 \mathrm{MW}$ to $536 \mathrm{MW}$. The regional export is reduced by that amount, and the extra dispatch is picked up far to the west. Otherwise, all the generation was left as is. This was still an extremely stressed system, but the loading on the St. Onge line was reduced to the static thermal rating. This would probably be the minimum action taken by a system operator. It has nothing to do with the fact that the power generation is from a wind power plant. It is interesting to note that the flow northwest toward the Lookout substation was reduced to a quite low level. The power wanted to head toward Rapid City and past Osage toward the attractive east-to-west egress provided by the Gateway projects.

This new power flow condition was called "Extreme-LW," which is not to be confused with the Hi-Mix, which was much lower stressed. 


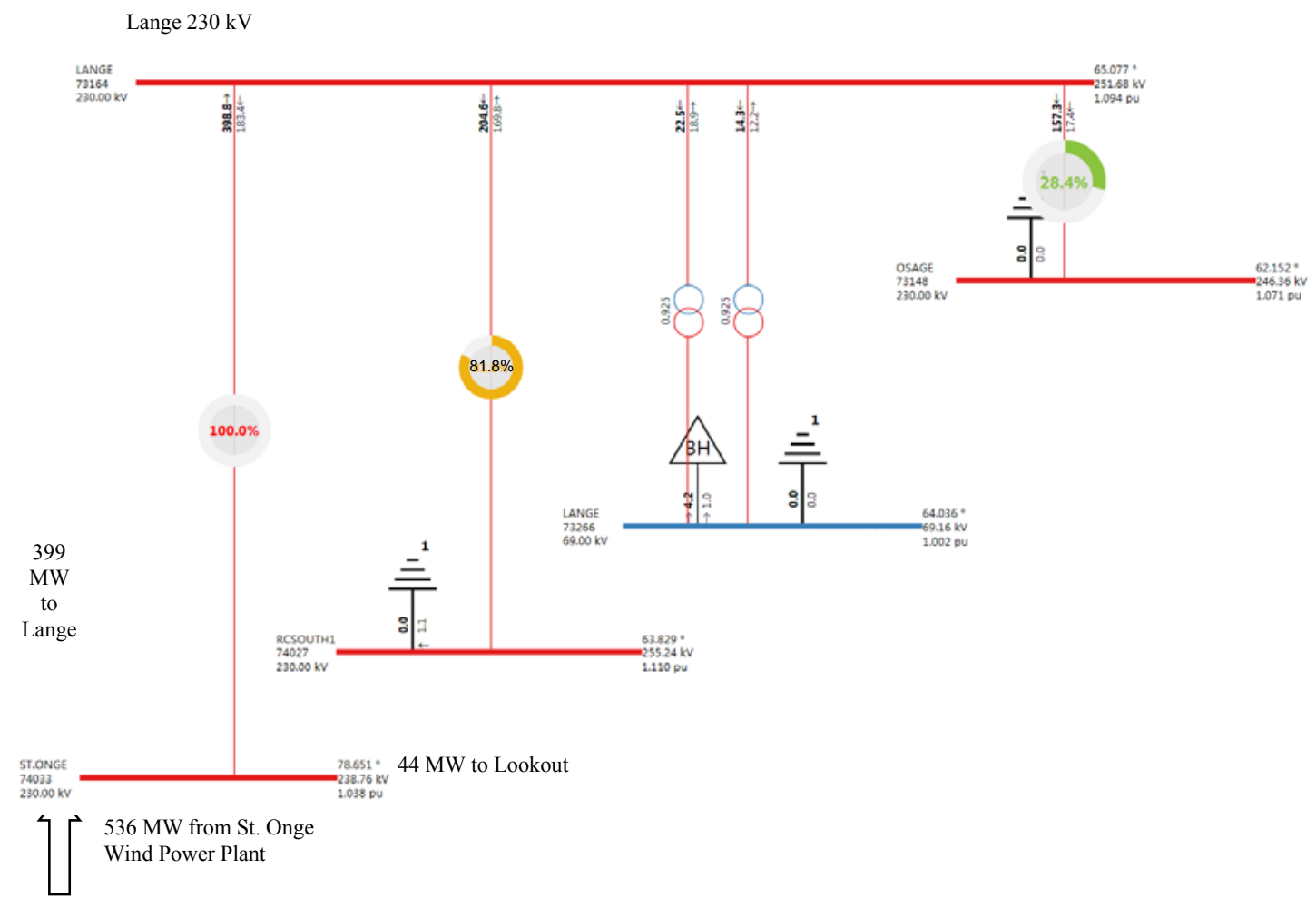

Figure 33. Reduced stress at St. Onge

When this reduced stress case was subjected to the Aeolus fault, without changing the LVPL settings from their modeling default, the system still collapsed during the fault. This is shown in Figure 34, in which the transmission bus voltages in Area 73 (WAPA RM) still collapsed (slightly more slowly than before). Similar behavior was observed in Area 65 (PACE). Clearly, although the output of the St. Onge plant is important to the regional dynamics, the collective behavior of all the wind power plants in the region remains critical. Dynamic reduction of power during the fault from the other wind power plants in the vicinity was still needed to save the system from collapse. 


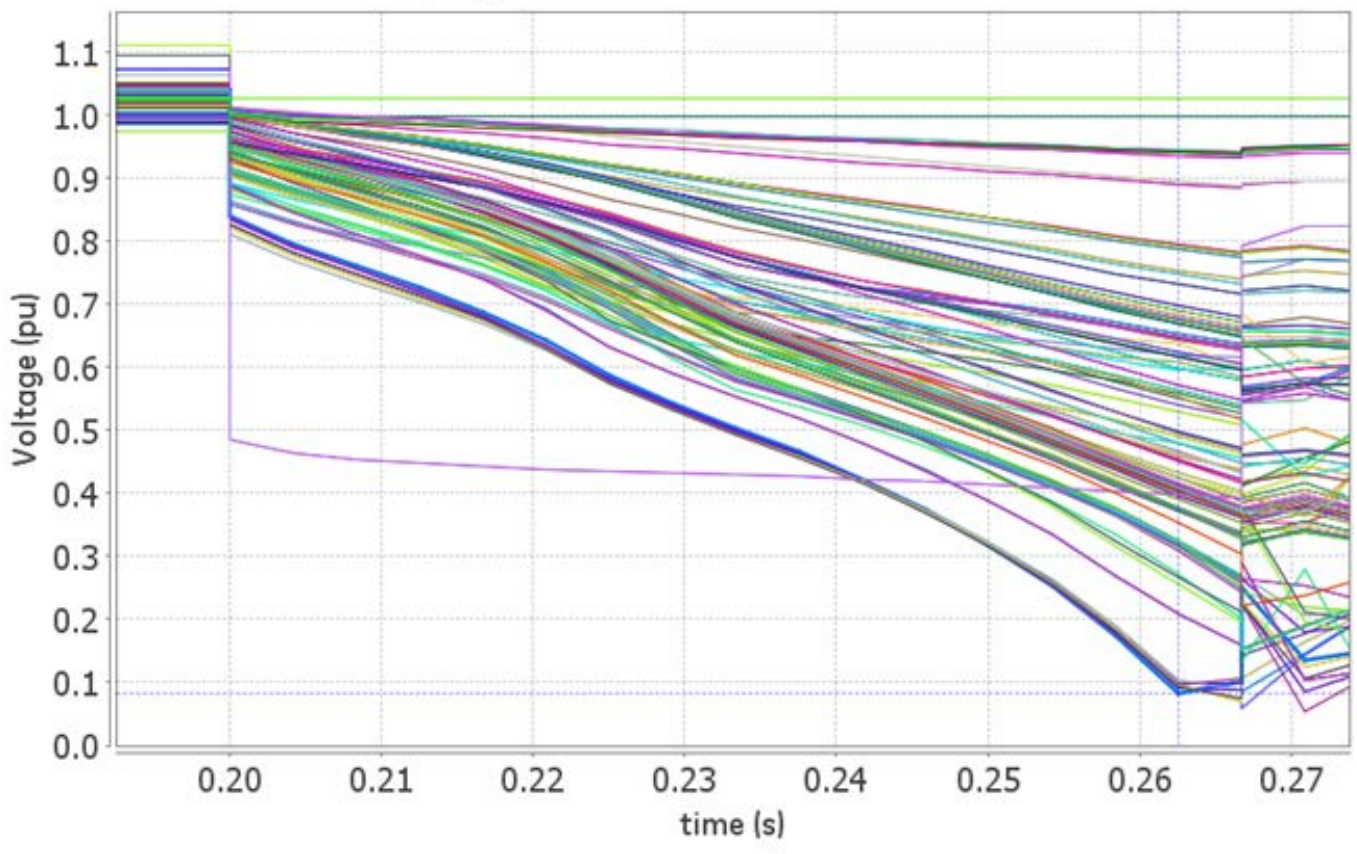

Figure 34. Reduced stress but limited LVPL_bus voltages in Area 73 (WAPA RM)

In the next sequence, the aggressive LVPL that was used in the Extreme cases was applied to the reduced stress condition. The relevant block diagram from the model is shown again in Figure 35 , with some additional annotations. In this case, and in the next sequence, the LVPL settings were adjusted. The default settings, which failed to stabilize the system above, are shown in blue - with the current completely suppressed only when terminal voltage dropped below $50 \%$. The "aggressive" control will completely suppress the active current when the terminal voltage drops below $85 \%$. This control is nearly a binary "bang-bang" control. These are well known to be effective, and they are equally well known to be difficult to get right and to avoid unintended consequences. The recovery ramp rate (rrpwr) to control how fast the current comes back up after being suppressed was also being adjusted. 


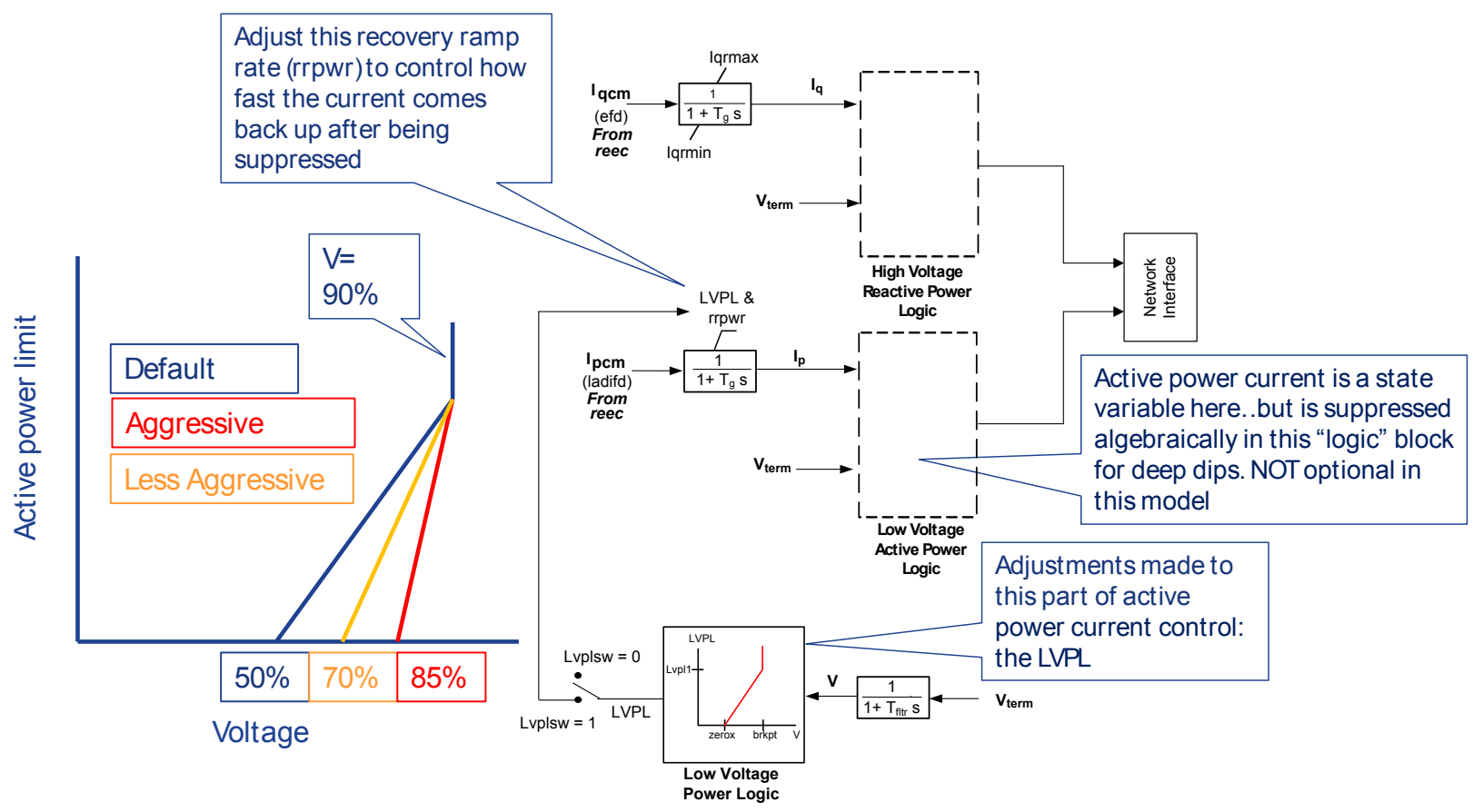

Figure 35. LVPL variations

The system performance with the aggressive LVPL is shown in Figure 36. The system survived the fault, and the recovery was mostly satisfactory, with the system returning to a satisfactory post-fault near-equilibrium within approximately $1 / 4$ of a second. There were a few quite fast (approximately $4 \mathrm{~Hz}$ ) oscillations that were well damped. Some details of the active current are shown in Figure 37. Again, current suppression was faster on some plants than others, depending on whether the algebraic limitation or the LVPL caused the suppression.

Post-fault-clearing dynamic overvoltage, as shown in Figure 34, was less severe than in the extreme case (Figure 23), but the swing was still cause for some concern. The details of the terminal conditions for the St. Onge plant are shown in Figure 38. According to this model the terminal voltages were limited to $120 \%$ for less than $100 \mathrm{~ms}$, so they should have been inside of the high-voltage ride-through criteria in the North American Electric Reliability Corporation PRC-024 standard. 


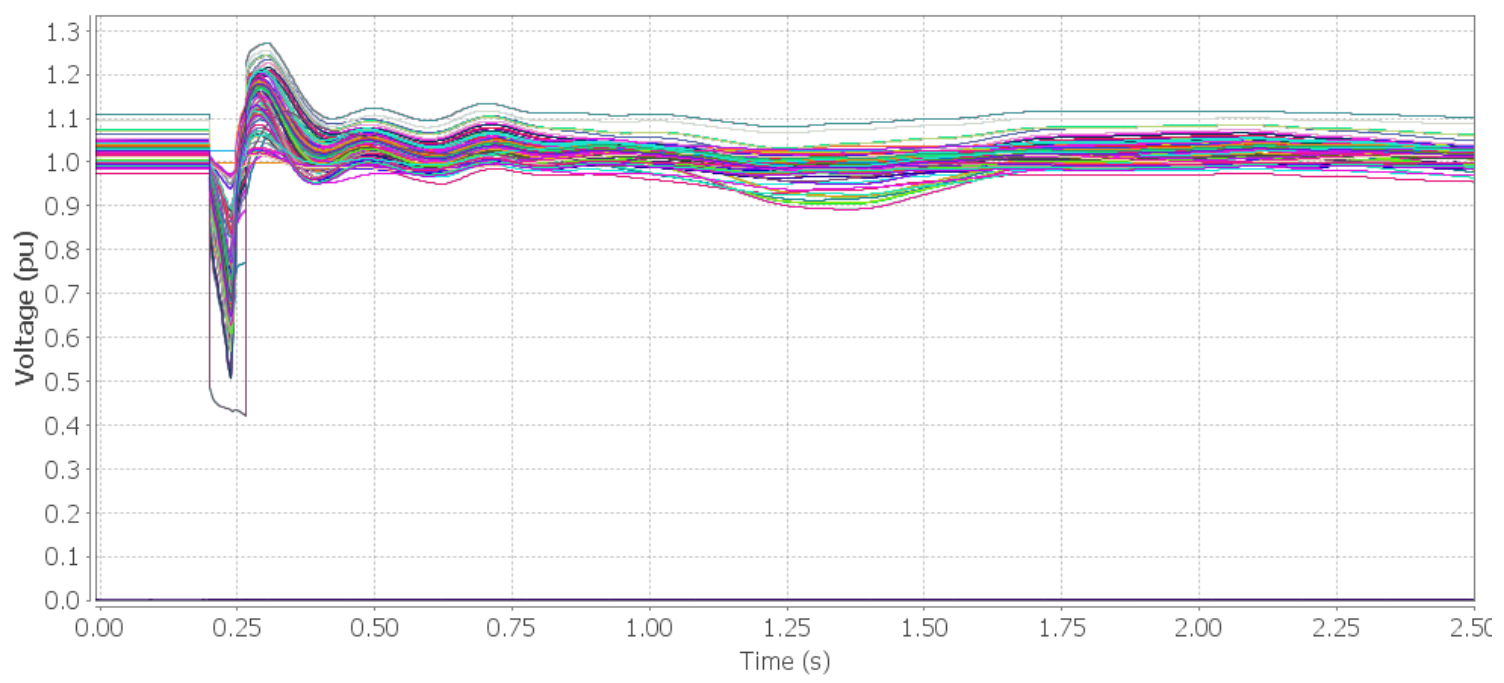

Figure 36. Voltages in Area 73 (WAPA RM) for reduced stress with aggressive LVPL

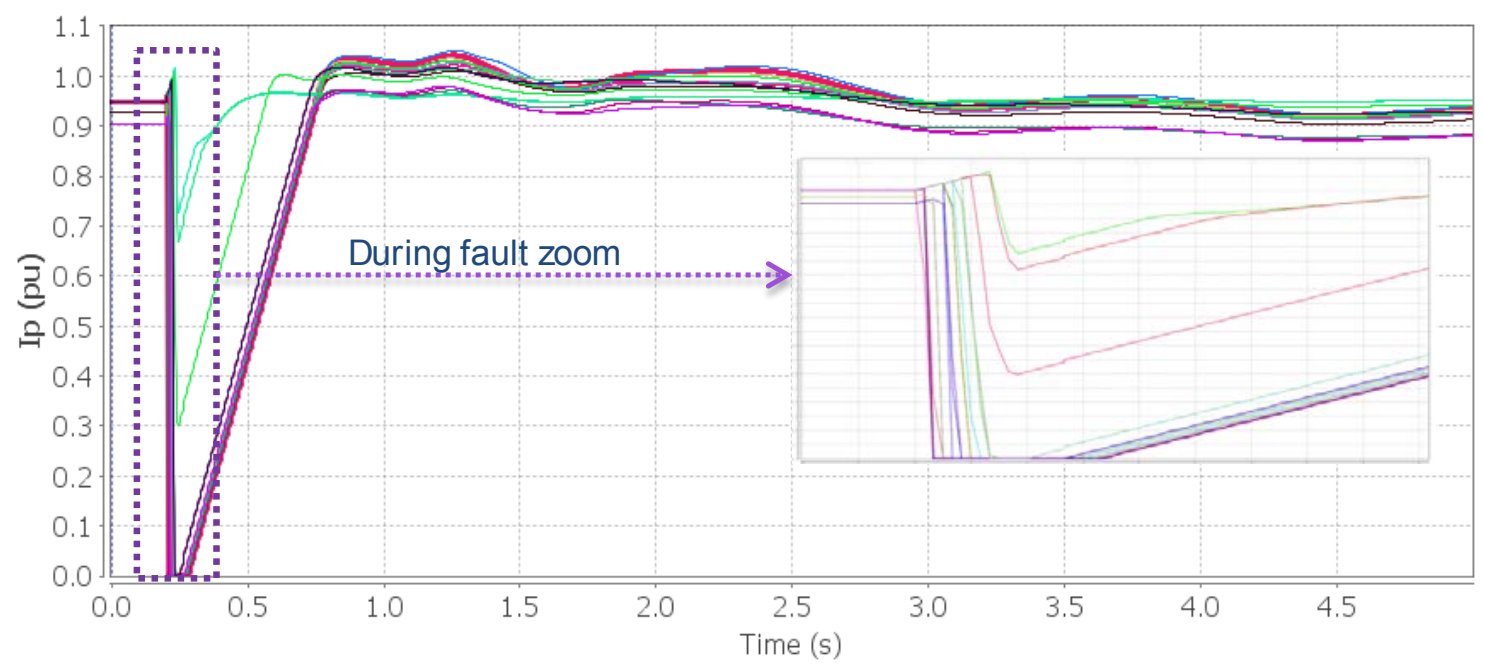

Figure 37. Active current on WTGs in Area 65 (PACE) 


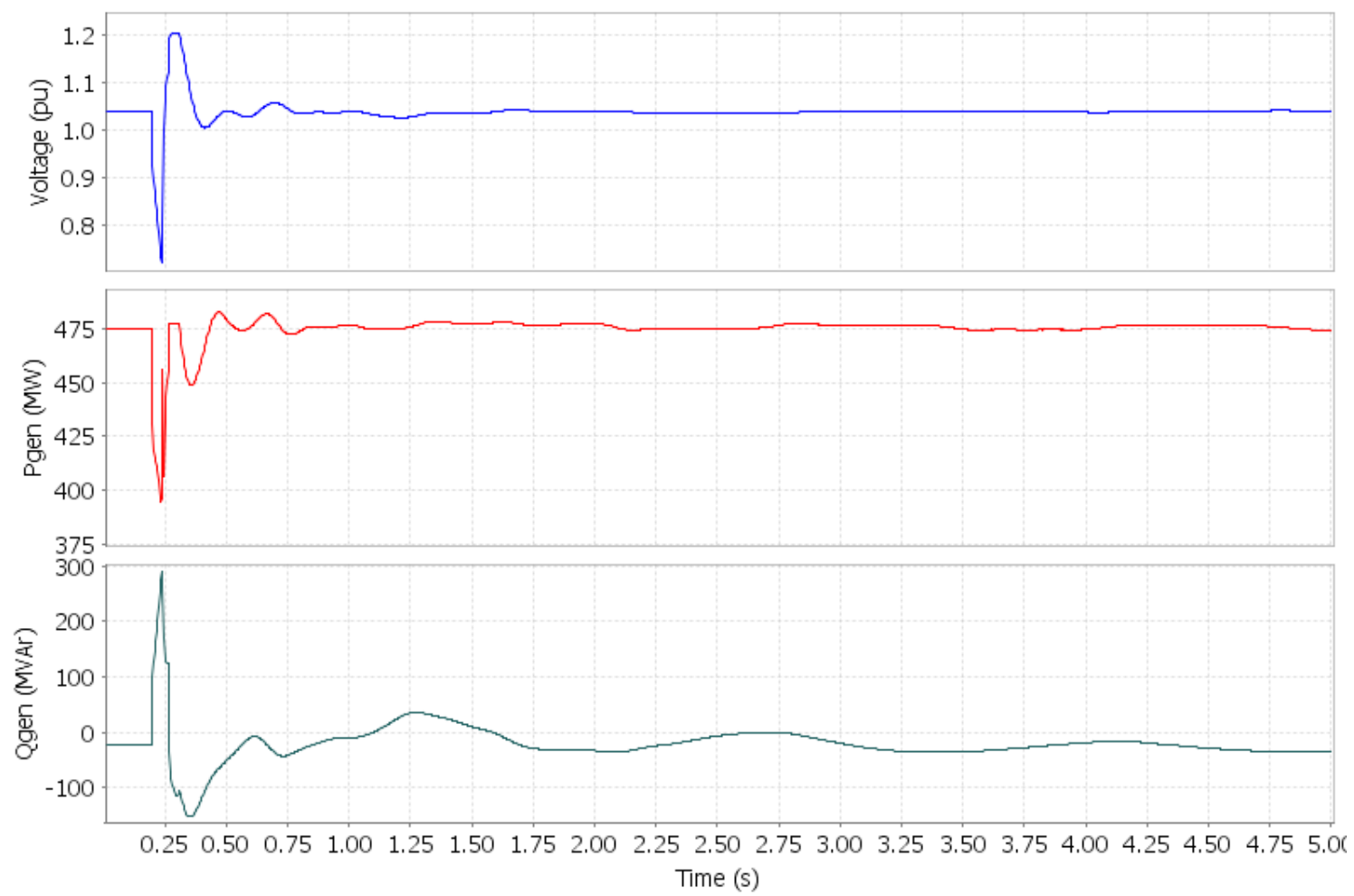

Figure 38. Performance of St. Onge wind power plant for reduced stress case

\subsubsection{LVPL Sensitivity Analysis}

As noted, the LVPL controls used in the case reported above were aggressive, i.e., gains on active current reduction were high. Here, a case with somewhat less aggressive gain (zerox = 0.7 ,the orange characteristic in Figure 35) that suppressed current when the terminal voltage dropped below $70 \%$ was tested, with the expectation that such a control will be more robust (and less prone to unintended consequences).

The resulting bus voltages in Area 73 (WAPA RM) are shown in Figure 39. The system still survived the fault and returned to stable operations. During-fault voltages were a little worse, and the high frequency swings in the voltage were a little less damped. Overall the system performance was satisfactory, although the immediate post-fault voltage swing was still rather high.

The reactive power delivery is shown in Figure 40. The overvoltage was caused by a combination of the reduction in active power causing the system to swing up the nose curve (as shown above) and the finite time required for the reactive current to drop following the rise in voltage that is apparent in this figure. In addition to that, the slow ramping of some WTGs, as shown in Figure 37, had the effect of reducing power flowing along several lines immediately after the fault cleared. Therefore, as voltages climbed in the post-contingency response, shunt capacitive compensation on those areas had a higher impact because of the reduced flows imposed by the slowly ramped generation. This is consistent with the PV curves shown in the voltage stability discussion. 


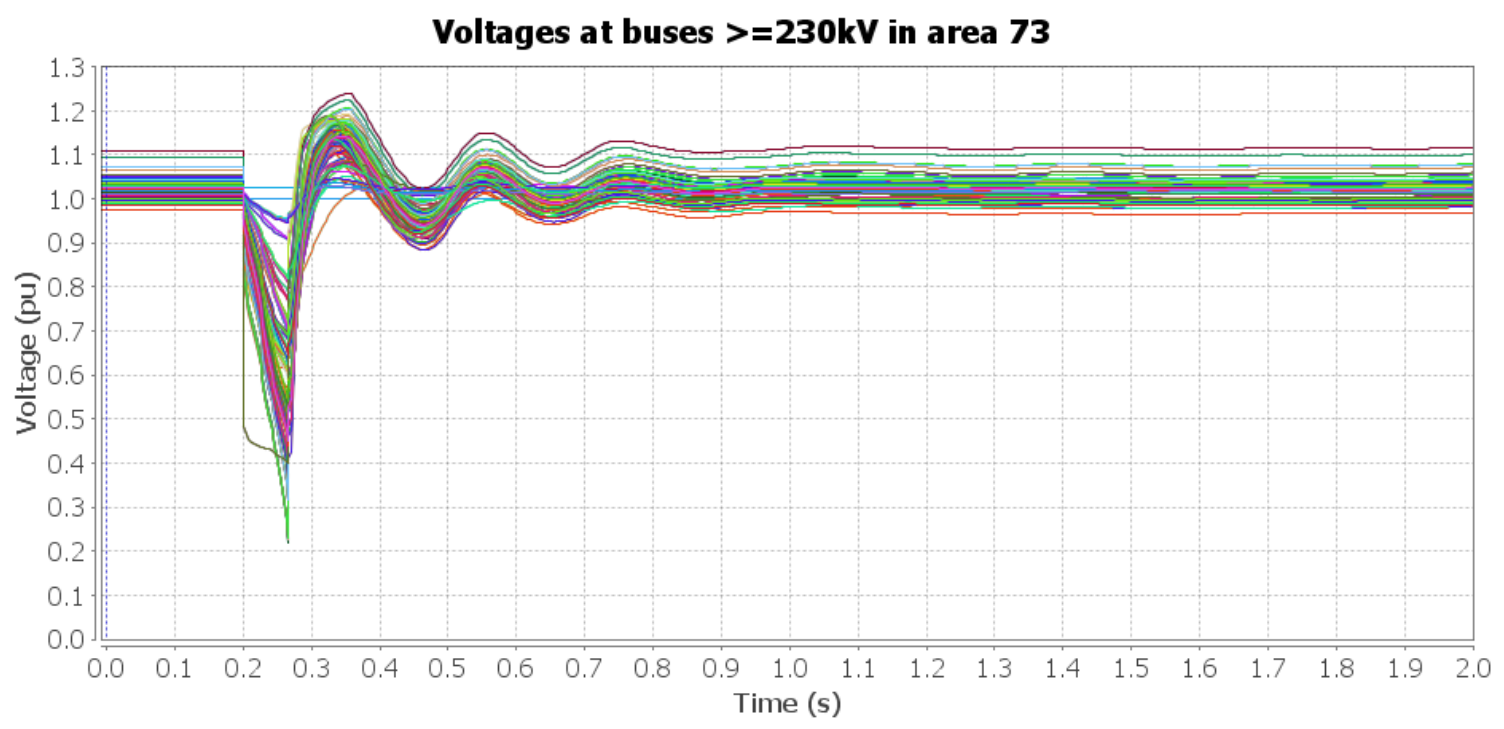

Figure 39. Less aggressive LVPL for the reduced stress extreme case

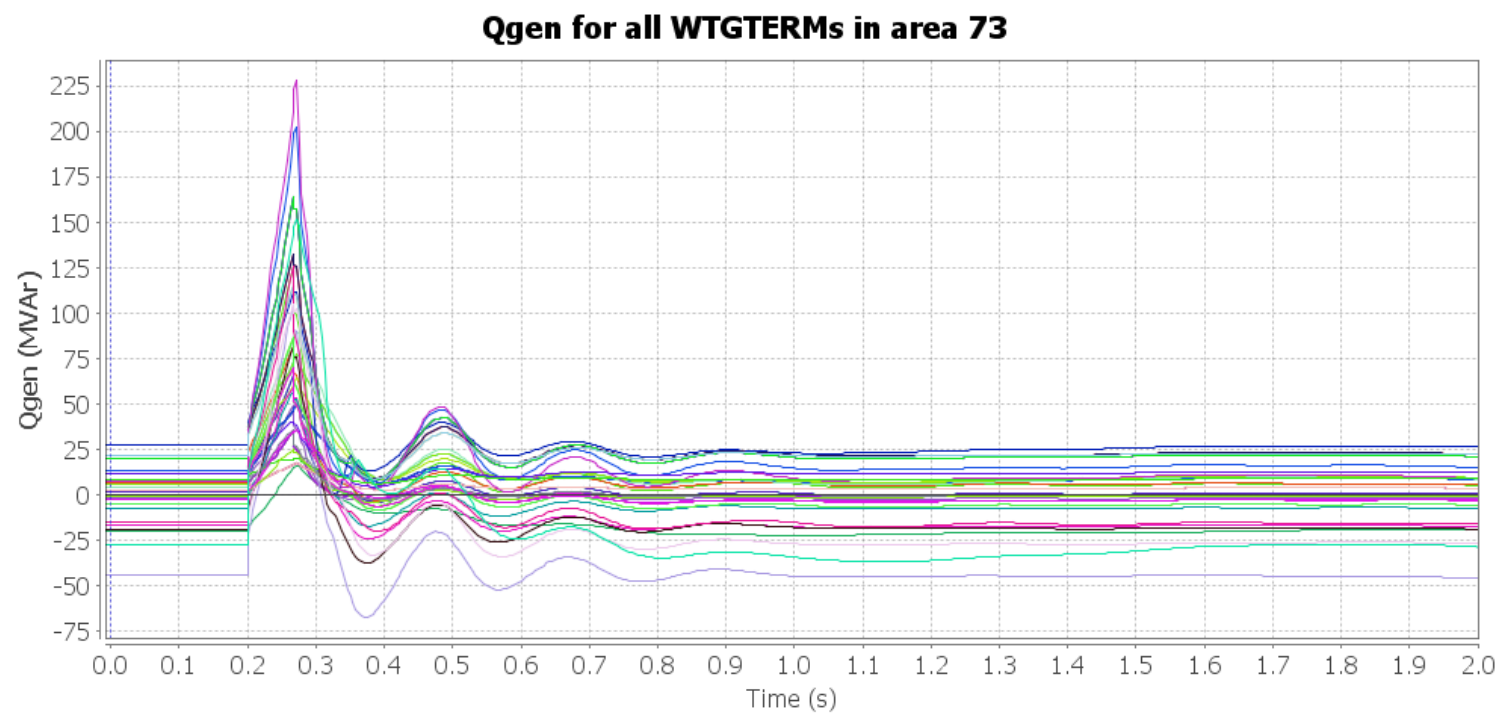

Figure 40. Less aggressive LVLP—reactive response from WTGs in Area 73 (WAPA RM)

\subsection{Synchronous Generator Response at St. Onge}

A comparison of system performance to a synchronous generator helps illustrate the difference in system dynamics. In this section, the St. Onge wind power plant was disconnected. Instead, a synchronous thermal plant of the same MVA rating and at the same dispatch as the wind power plant is added.

In Figure 41, the 1,200-MVA synchronous St. Onge generator was dispatched at $832 \mathrm{MW}$.

The unit and the system were stable during the fault. The drop in the active power- the active power suppression (due to Park's equations) - during the fault was substantial. The rapid postfault power recovery to decelerate the machine did not collapse the system. The damping was 
rather poor, but there was no attempt to tune the power system stabilizer. The reactive power was healthy upon recovery.

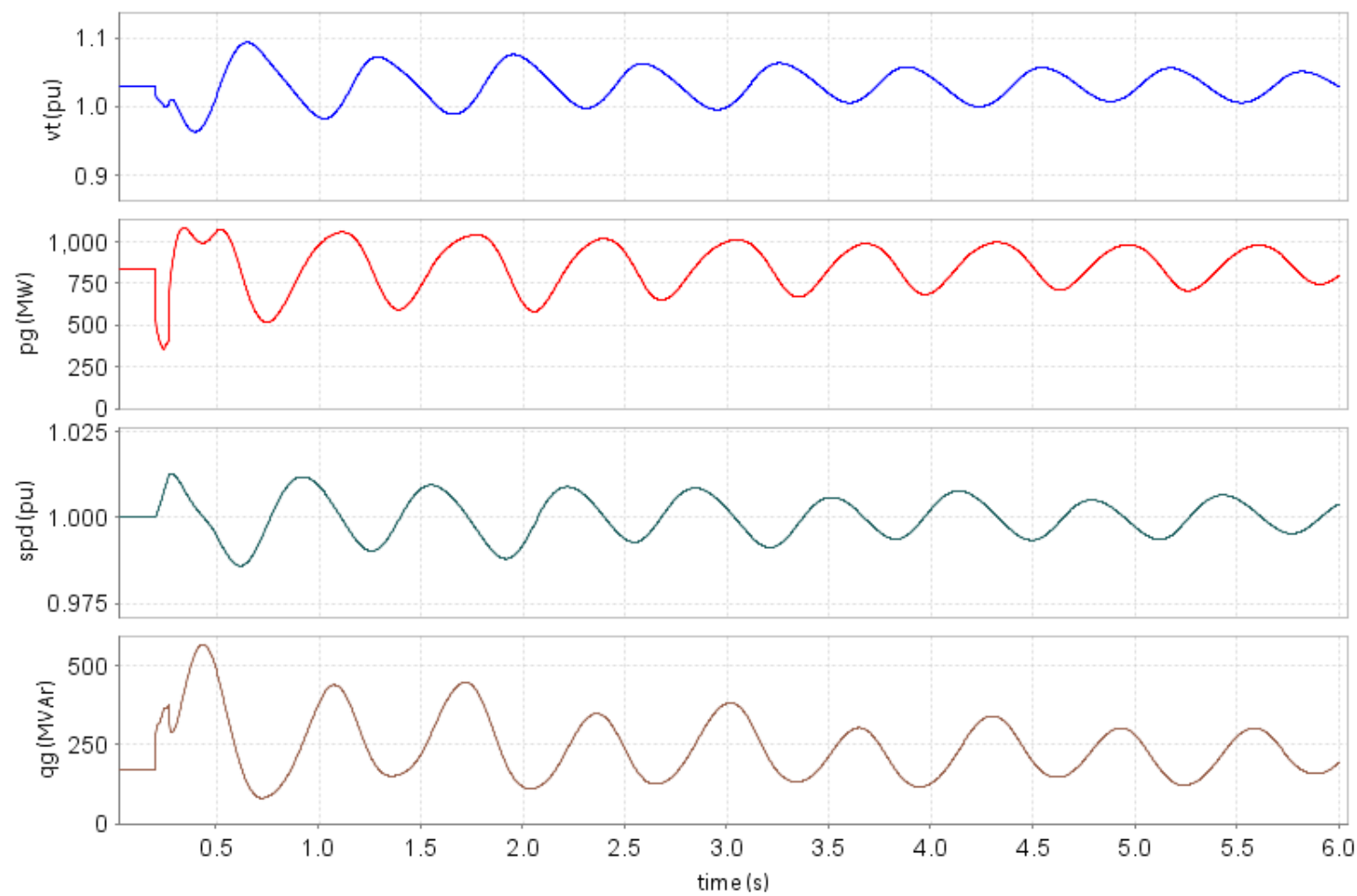

Figure 41. Synchronous generator at St. Onge-extreme dispatch

All of the transmission voltages in Area 73 (WAPA RM) are plotted in Figure 42. The grouping of the voltages shows that the oscillations were relatively localized. The machine speeds of the synchronous generators in the area are shown in Figure 43. The units in the Rapid City area, especially St. Onge, were swinging with the small hydropower units (e.g., Alcova) immediately to the west. The period of oscillation was on the order of $1 \mathrm{~Hz}$, which is typical for this type of regional or subregional damping problem. Damping problems of this type are commonly addressed by PSS tuning. Under extreme stress, such as here, PSS alone might not add enough damping given the unrealistically high rating of the fictitious machine at St. Onge. PSS tuning is outside the scope of this project. 


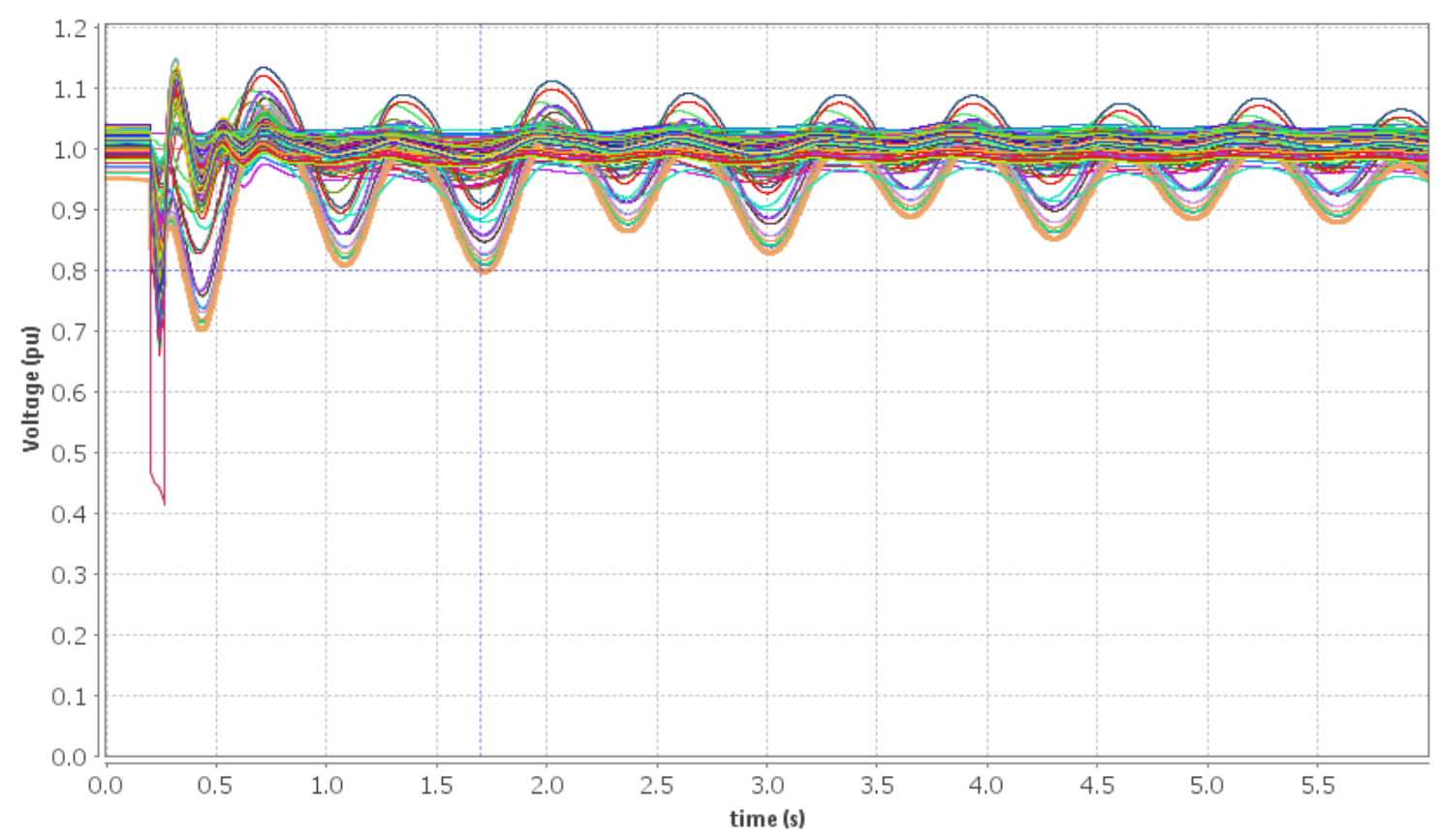

Figure 42. Area 73 (WAPA RM) bus voltages with synchronous generator at St. Onge

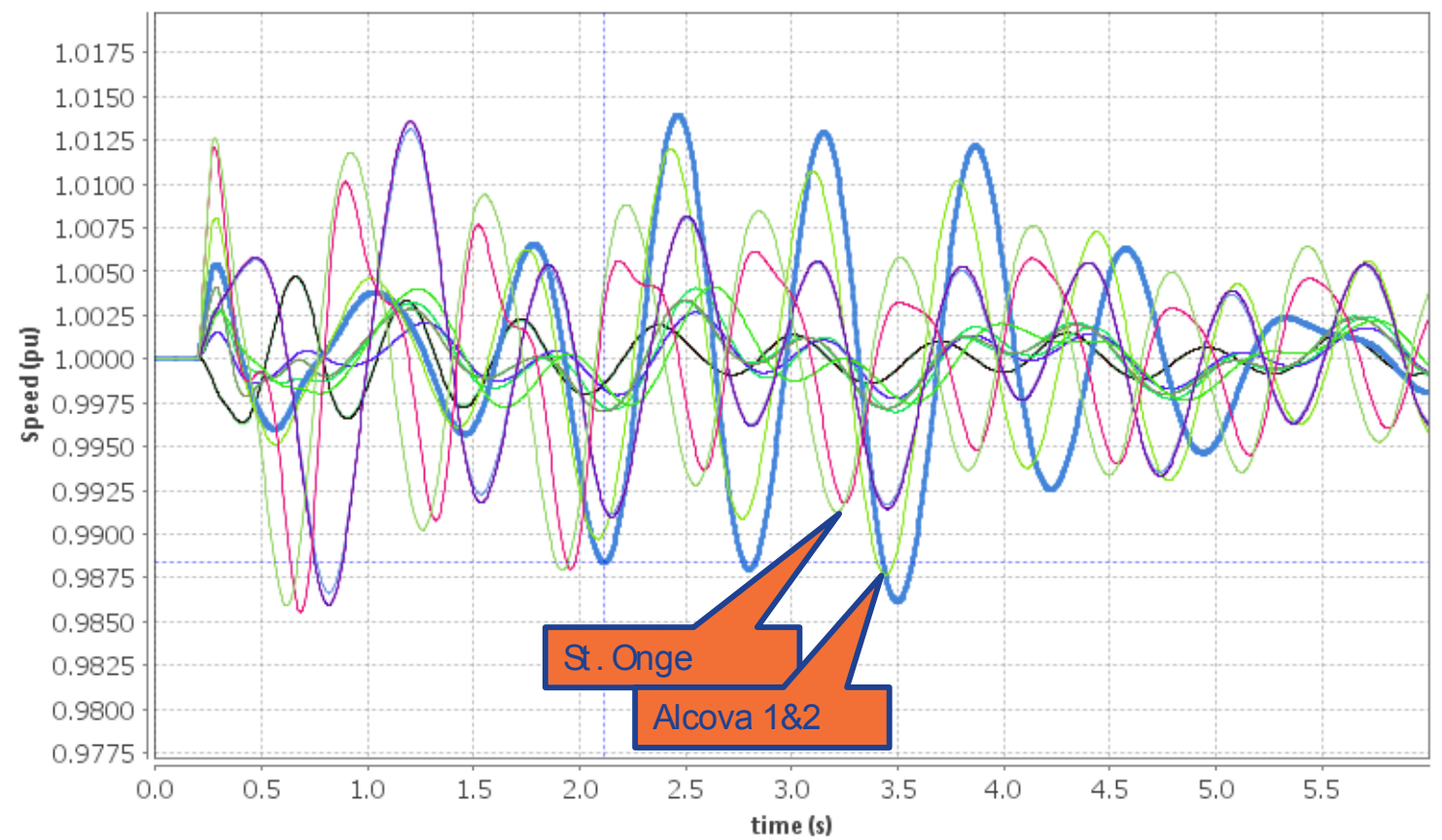

Figure 43. Synchronous machine speeds in Area 73 (WAPA RM) for the synchronous St. Onge case 


\subsubsection{Reduced Dispatch at St. Onge}

When the dispatch of the synchronous St. Onge plant was reduced to the level used in the dispatch for the reduced stress case, the stability was improved. In Figure 44, the machine swings are shown to be reasonably well damped.
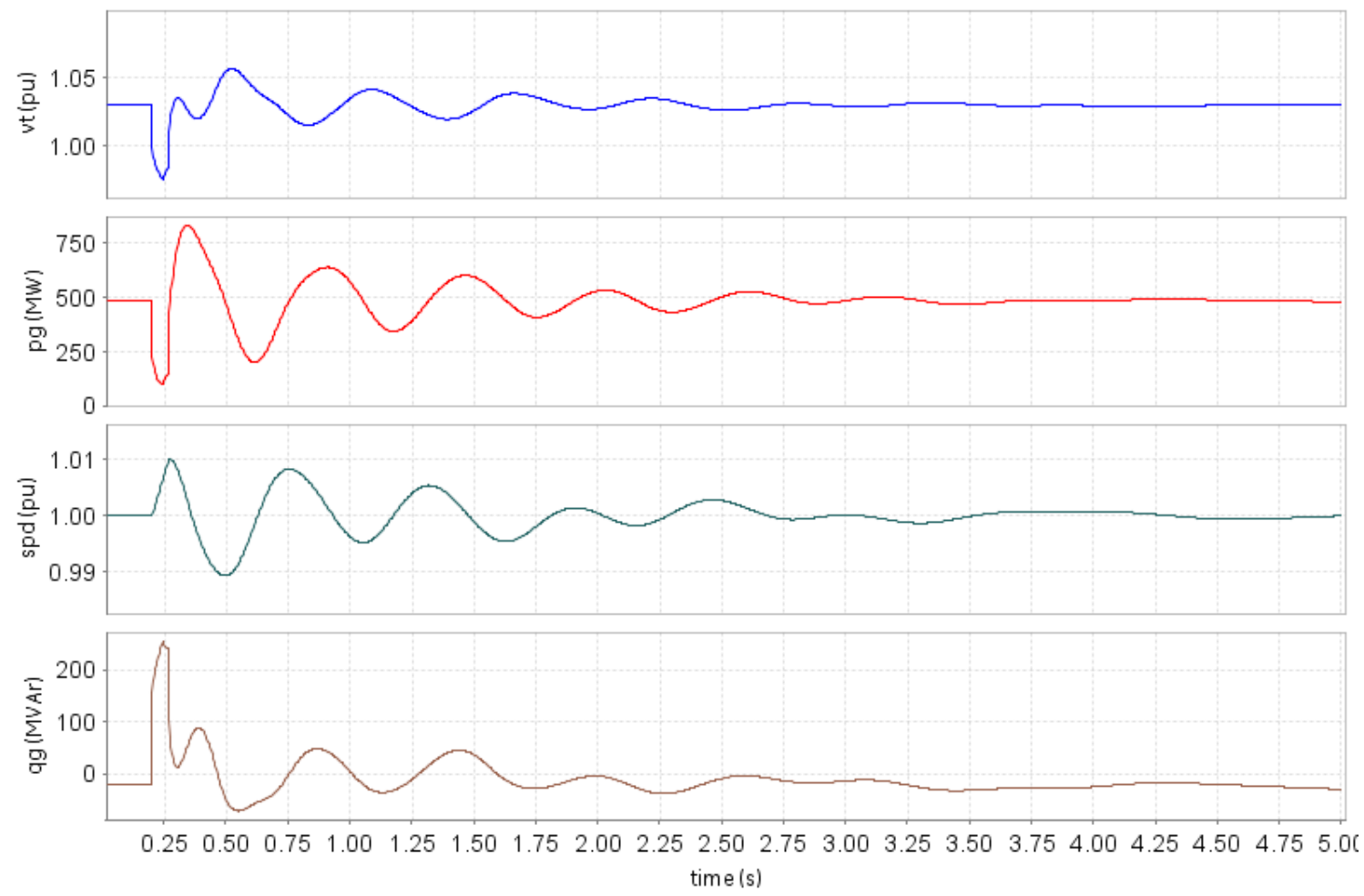

Figure 44. St. Onge synchronous machine response-reduced dispatch case 
The relative performance of the two cases was significantly different, as shown in the comparison plots of Figure 45. In particular, the voltages throughout the region both during the fault and immediately following the fault were better. This appears to be a situation in which both the inertia and the flux state-variable modeling of the machine were beneficial to the local stability. As observed in the previous study, having some synchronous machines in key areas of the stressed system is beneficial to stability.
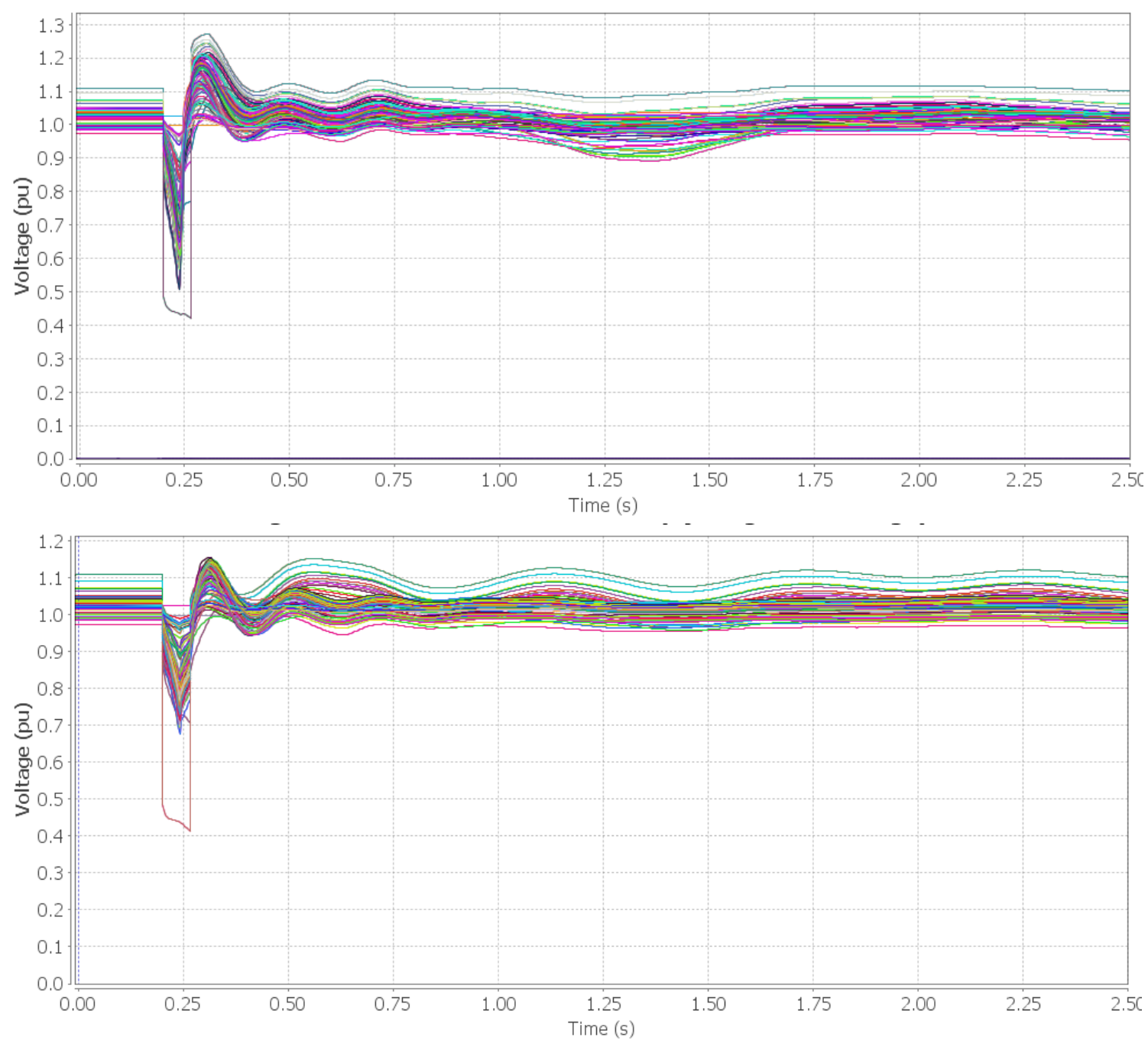

Figure 45. Voltage swing comparison: WTG (top) compared to synchronous generator (bottom) 


\subsection{Sensitivity to Wind Turbine Model}

In the sequence of cases above, it was stated that the settings of the control feature can be adjusted to change the performance. In particular, setting the LVPL, a feature specifically intended to improve system stability, can have a substantial impact.

However, other modeling assumptions can impact system performance, particularly in the very fast time frames, i.e., a few cycles. One important aspect of the generator model is its representation of the generator interface to the network. The new, WECC REMTF, secondgeneration, generic model has two types of network interfaces:

- Pure current injection: (variable $\mathrm{X}$ ” is zero)

- Hybrid: Current P \& Norton Q (variable X" is $\neq$ zero)

These two interfaces originally emerged (in modeling documents by GE) as being assigned to Type 3 (doubly-fed) and Type 4 (full-converter) machines. As control features have evolved, and our understanding of the critical aspects of generator performance in these fast time frames has improved, that distinction has become less meaningful and less appropriate. In the balance of this section, the more precise description based on the interface, rather than the purported machine type assignment, will be used.

Later in this section, a third network interface will be presented that uses a full Norton connection to the network equations. This interface is used in the new, specific, GE original equipment manufacturer (OEM) model, recently developed to reflect both new features intended to improve weak grid performance and more accurately capture the behavioral characteristics of GE's doubly-fed machines in weak grid applications.

The modeling of the grid interface for wind generators is complicated by the fact that the flux time constants involved are small. Approximations are needed, just as they are with all other component models in stability programs. Maintaining acceptable numerical stability is also necessary.

A comparison of the hybrid and pure current interface performance is shown in Figure 46. The two models do not allow for identical parameters, but the case comparison is for approximately equal control settings. The event is the Aeolus fault in the Hi-Mix case, and the plots are for one equivalent unit at St. Onge.

The pure current injection model delivered less reactive current across voltage discontinuities. The voltage sag was worse, so the regulator tended to wind up and then overshot. The algebraically limited reactive current during high-recovery voltage caused the terminal voltage to be limited to $120 \%$. Overall, the pure current interface performed noticeably worse. 

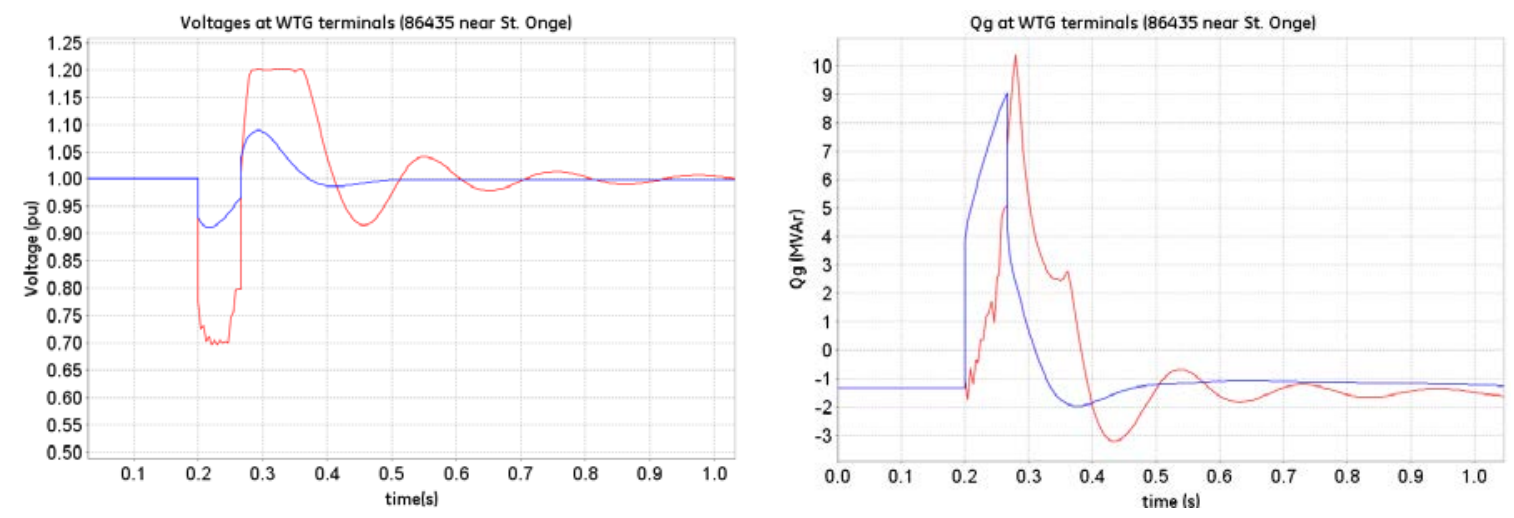

- $\quad$ Blue = Hybrid Current P\& Norton Q

- $\operatorname{Red}=$ Pure Current $P \& Q$

Figure 46. Comparison of WTG network interfaces

\subsection{Sensitivity to Wind Turbine Functionality}

All of the results shown so far as "base" or "default" originated from one manufacturer's relatively high-functionality WTG and wind power plant controls as they would have been implemented for "normal" (i.e., not weak grid) applications.

The implementation was conservative, in that the "reaches" of the wind power plant voltage controls were deliberately limited (i.e., they stayed within the new plants). This is a tuning option, but it not very important in the short time frames of primary interest here. Special active power controls, such as those used for the frequency response work in the previous WWSIS-3 study, were not enabled in these cases.

The plants were assumed to be compliant with present U.S. grid code, which requires fault ridethrough and closed-loop voltage regulation. However, the present codes do not specify:

- Weak grid controls - i.e., a minimum composite short-circuit ratio (SCR) down to which the WTGs must be stable

- The speed of wind power plant voltage regulation

- Control over the recovery of active power following a disturbance

Indeed, some non-North American grid codes actually demand fast active power recovery. These may make some sense in stiff systems, but, as shown here, that is problematic in weaker, exporting grids.

\subsubsection{Reduced WTG Functionality}

All WTGs reduce active power during very deep voltage depressions as a consequence of topology and current control. But, so far, we have shown controls that deliberately suppress active current recovery. Figure 47 shows various reduced functionality controls that were tested in the Hi-Mix case with pure current injection network interface models. The first case, shown in 
red, is with the controls as presented above-i.e., with fast voltage regulators and with a slowed recovery of active current. In the second case, shown with blue traces, the WTG voltage regulators were slowed by an order of magnitude. In the third case, the slow voltage regulator was combined with the fast recovery of the active current. This case is particularly interesting because it showed fault-induced delayed-voltage recovery behavior (NERC 2009). This is similar to the behavior that recovering load currents cause when there is inadequate dynamic voltage support. The phenomena here were clearly similar, except the poor voltage behavior was from an injection of active power rather than the high consumption of reactive and active current from motor loads.

The slower voltage regulator case combined with too-rapid active current recovery is a credible concern. Some wind turbine OEMs have only central, plant-level voltage control that is rather slow. WTGs intended for stronger grids tend to have fast active current recovery. These cases were run in the Hi-Mix scenario because they are known to fail in the Extreme case.
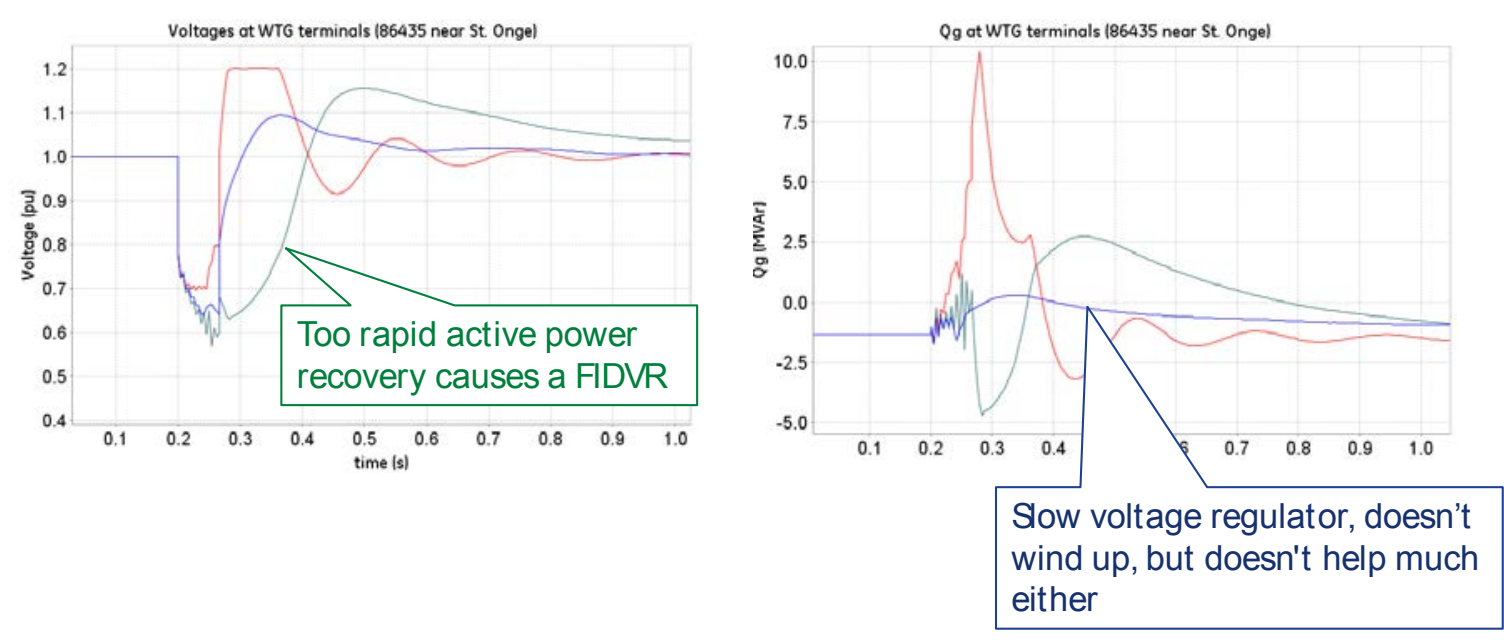

- $\operatorname{Red}=\sim$ same control settings as Norton/Current model

- Blue = with slow regulator

- Green = with slow regular and no ramp rate limit

Figure 47. Reduced WTG functionality

\subsubsection{A Manufacturer-Specific WTG Model}

WTGs, controls, and models are continuously improving. This is particularly relevant for weak operating conditions, such as those investigated in this exercise.

The WECC REMTF, second-generation, generic models were not intended for such weak systems. It is pessimistic (for GE WTGs) in the scenarios simulated in this study. However, GE recently developed a model that has additional details of new features for use in weaker grids.

This more detailed model is a better representation of the present-technology WTG performance in weak grids. The more detailed model is a full Norton network interface with fast current 
control loops as well as current controls that better handle the change in terminal conditions. For comparison, all of the wind power plants in the West that were added to build the highrenewables topology of both the Hi-Mix and Extreme cases from the Base case were replaced with this model. Plants existing in the original WECC case were left alone, as were all of the solar power plants.

Figure 48 shows the transmission voltages for the Extreme case (compare these plots to Figure 23). In this case, the during-fault system separation, the post-fault clearing overvoltage, and the nonlinear LVPL-induced oscillations were mitigated. The terminal voltage (shown in Figure 49) and the active and reactive power (shown in Figure 50 and Figure 51) were all well-mannered.

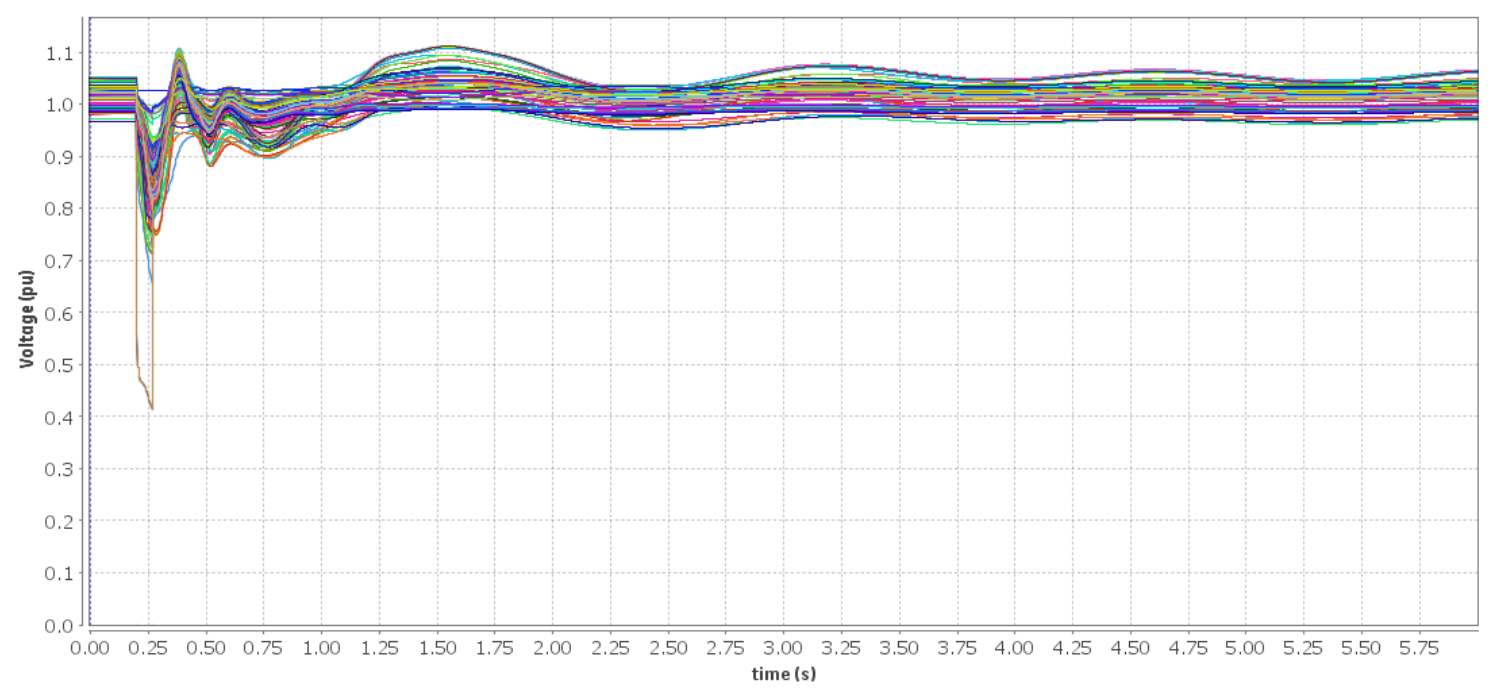

Figure 48. Area 73 (WAPA RM) voltage for Extreme case-OEM model

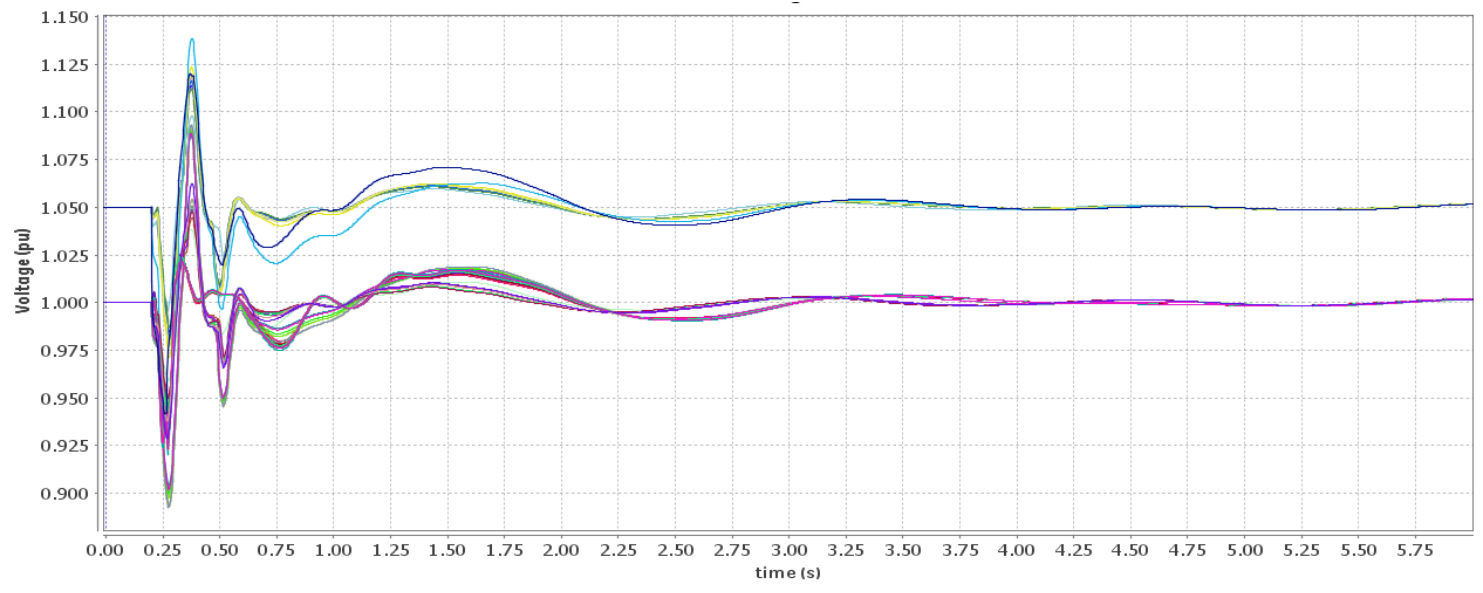

Figure 49. Area 73 (WAPA RM) terminal voltages (Vt) for Extreme case-OEM model 


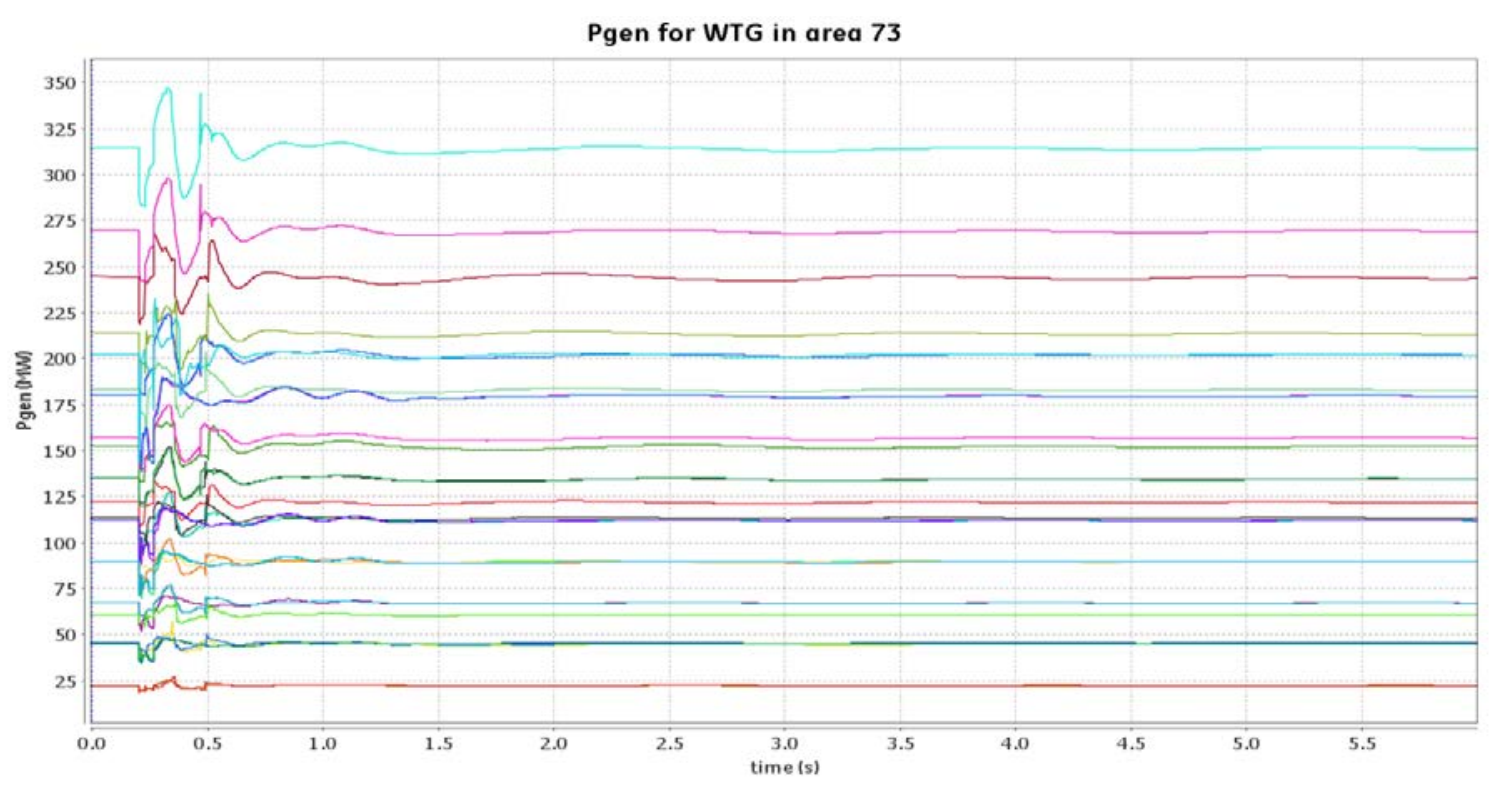

Figure 50. Area 73 (WAPA RM) active power-OEM model

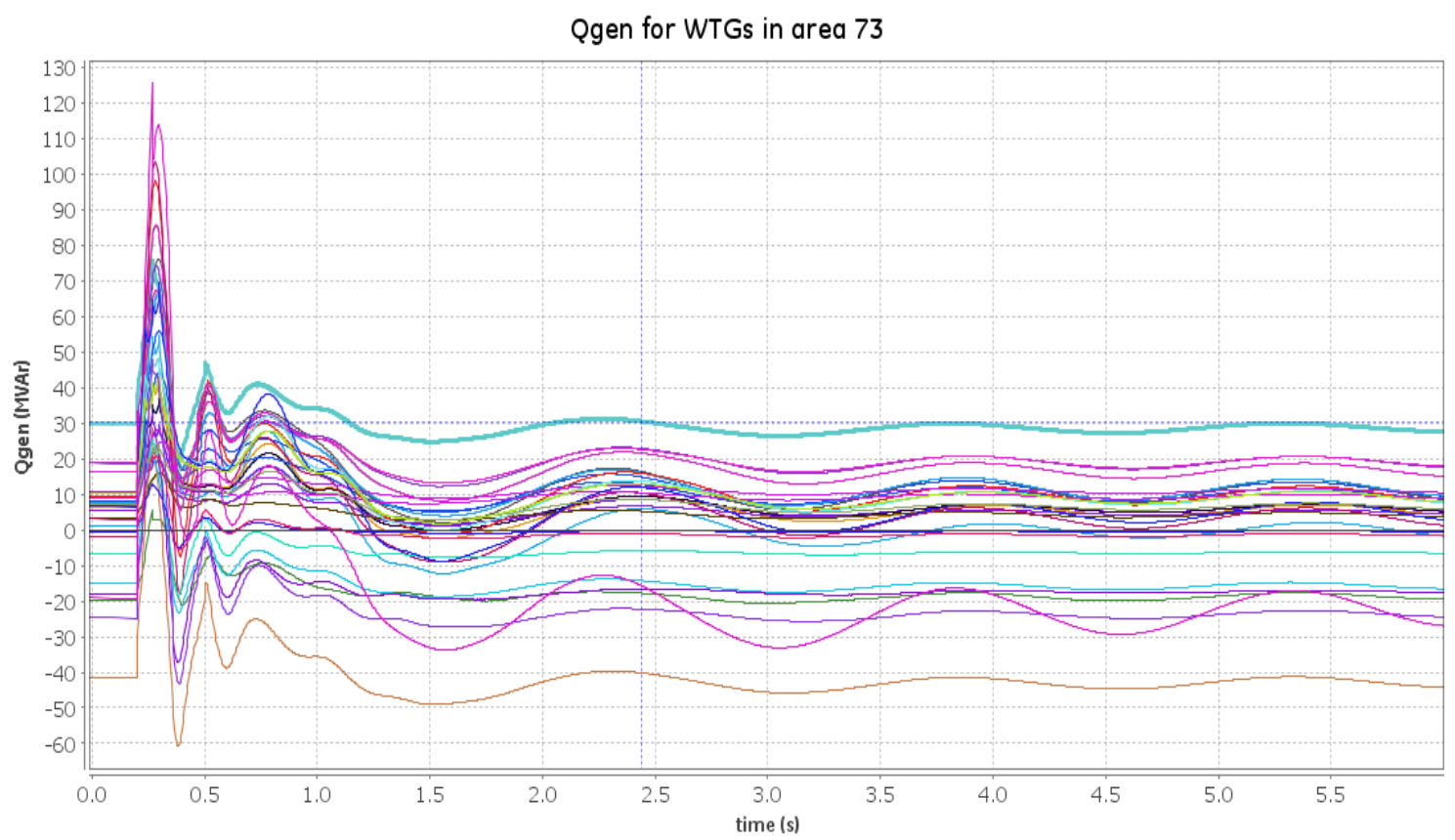

Figure 51. Area 73 (WAPA RM) reactive power-OEM model

\subsection{Examination of Load-Modeling Impact}

In WWSIS-3, the WECC composite load model was shown to have a profound impact on system stability. This section shows the results of selected investigations into load-modeling assumptions. The objective of the analysis was to further investigate the impact of different load models on Western Interconnection system performance. 
The results shed light on beneficial practices regarding load modeling and actions (e.g., undervoltage load shedding) to maintain system stability under high penetrations of renewables.

Two system loading conditions considered: the Extreme case and the Extreme case with reduced St. Onge dispatch (Extreme-LW).

\subsubsection{Composite Load Model}

The composite load model was developed by the WECC load-modeling task force (LMTF) to properly account for load dynamics during system events. The model, shown in Figure 52, is a mix of static load, frequency-dependent current, and motor loads (WECC 2012). The parameters can be tuned to reproduce past fault-induced delayed-voltage recovery (FIDVR) events. The load representation included a refinement developed by Bonneville Power Administration that takes into account the system loading conditions of different climate zones. The distributed rooftop PV generation added in the Hi-Mix and Extreme cases is shown in red and summarized in Table 1.

Motors in the model can be either one phase (AC load) or three phase (industrial motors). These motors include sufficient dynamic representation to capture stalling phenomena. Further, undervoltage tripping of these motors, to mimic contactor dropout, is possible. In WWSIS-3 and throughout the simulations presented so far in this report, this motor-tripping behavior was blocked. The intent of blocking this feature was to avoid masking stability behavior changes that are caused by the wind and solar additions.

Under-voltage tripping of loads is often beneficial from the perspective of transient stability, with the loss of load resulting in less system stress. However, the region of interest for this investigation is exporting, and the loss of load is not necessarily beneficial.

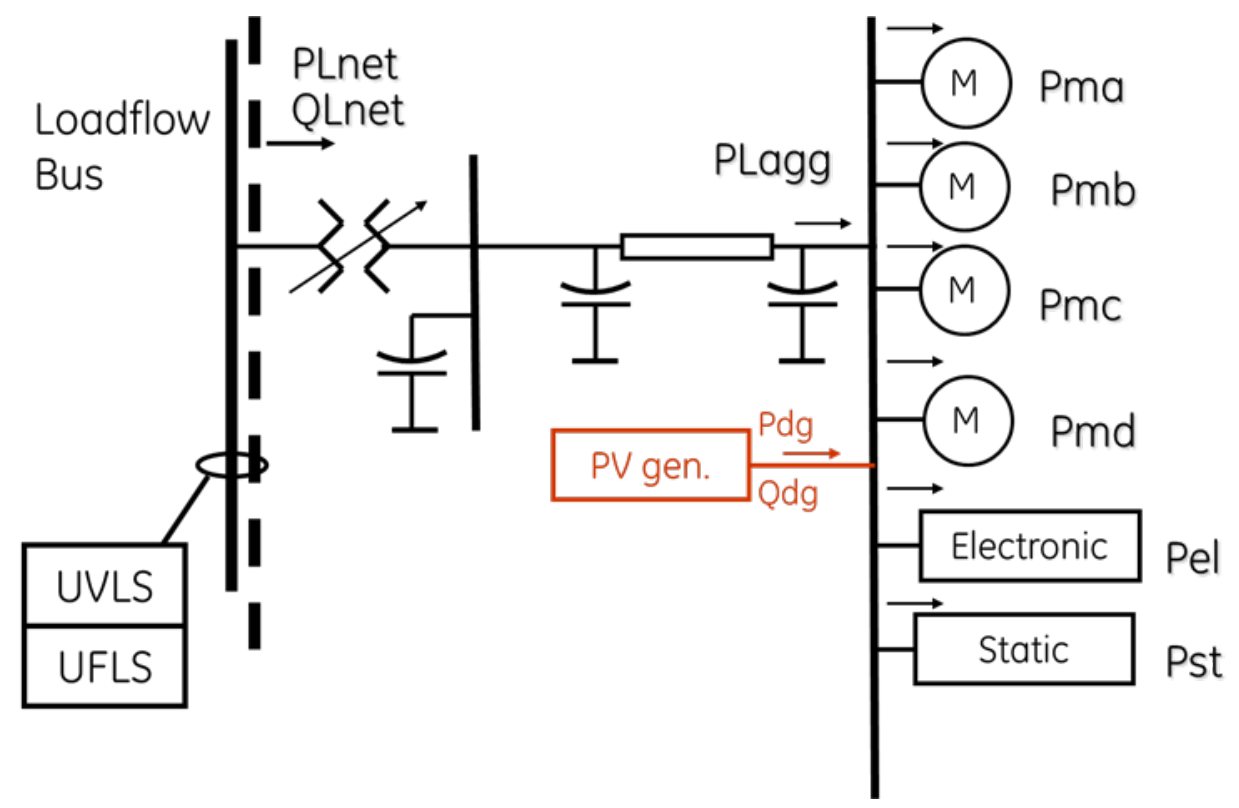

Figure 52. Composite load model 
Figure 53 shows the system voltages for the reduced stress (Extreme-LW) case with the tuned LVPL control on the WECC REMTF, second-generation, generic models. This case can be compared to results presented in Figure 39. The response was well damped, similar to the case without tripping. However, the behavior during the fault was more complex.
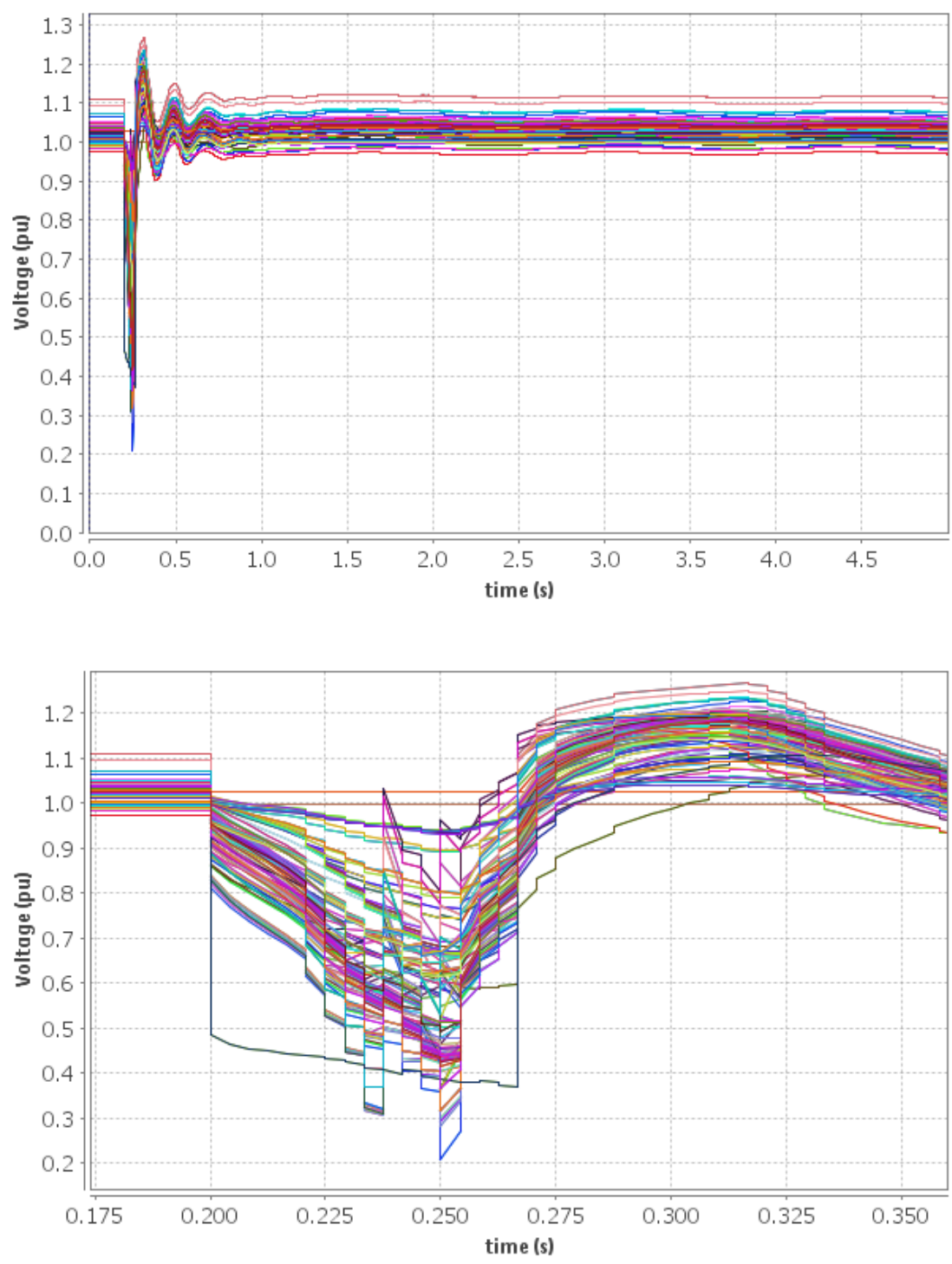

Figure 53. Voltages-composite load with motor tripping (detail bottom)

To examine that behavior, the response of a single load bus was plotted, as shown in Figure 54 . The Reno $230-\mathrm{kV}$ bus was sufficiently close to the fault. During the initial $20 \mathrm{~ms}$ of the disturbance, the trajectory was similar to that observed without motor tripping in the load models. But then, at each time step, there were discrete steps up or down in voltage driven by the tripping of the motors. The behavior is complex, because the system was right on the end of the PV curve. The PV curve was stressed by the active power being exported from the region. Consequently, when the local load was reduced by the individual load elements tripping, as shown in Figure 55, the active power term driving the system under the nose of the curve 
increased, depressing the voltage. However, the tripping of the load also reduced reactive power loading, as shown in Figure 56, which is a magnified view around the fault. This is beneficial for stability, and it can help with the overall system profile. The net result was the complex trajectory and the complex interaction with the local wind turbines shown in Figure 57.

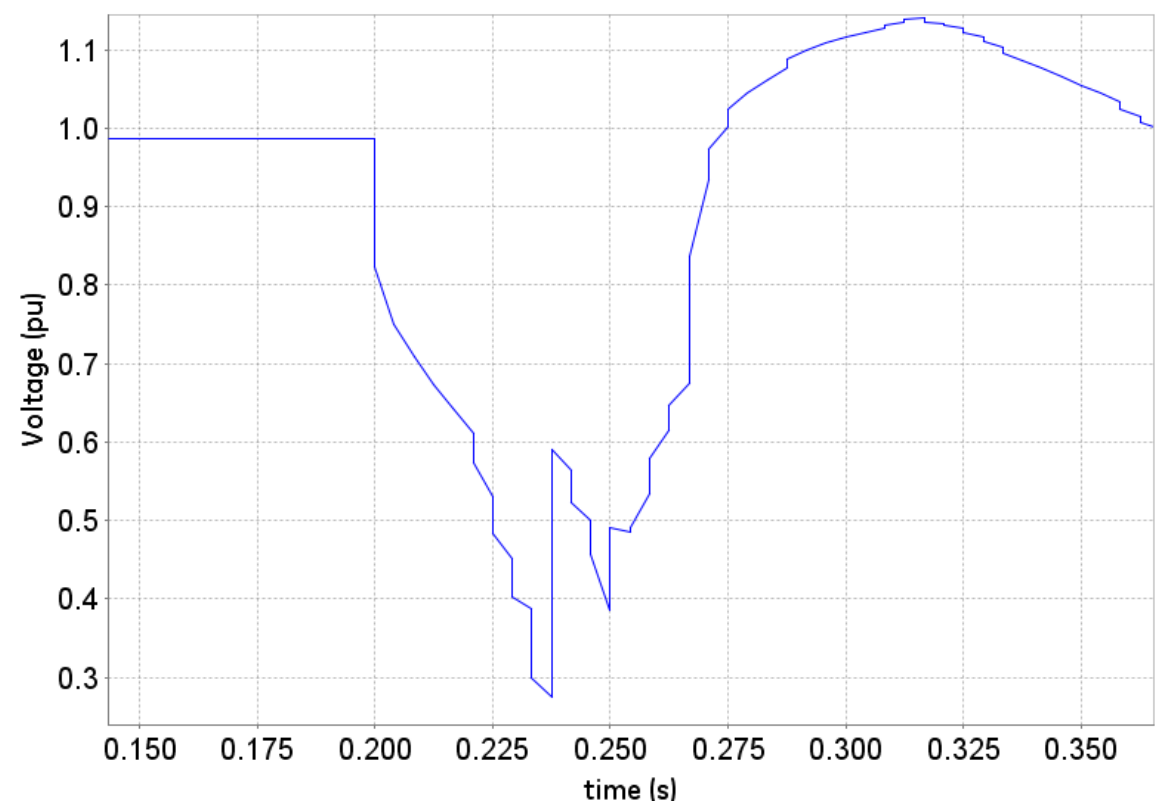

Figure 54. Voltage at load bus for a composite load with motor tripping 

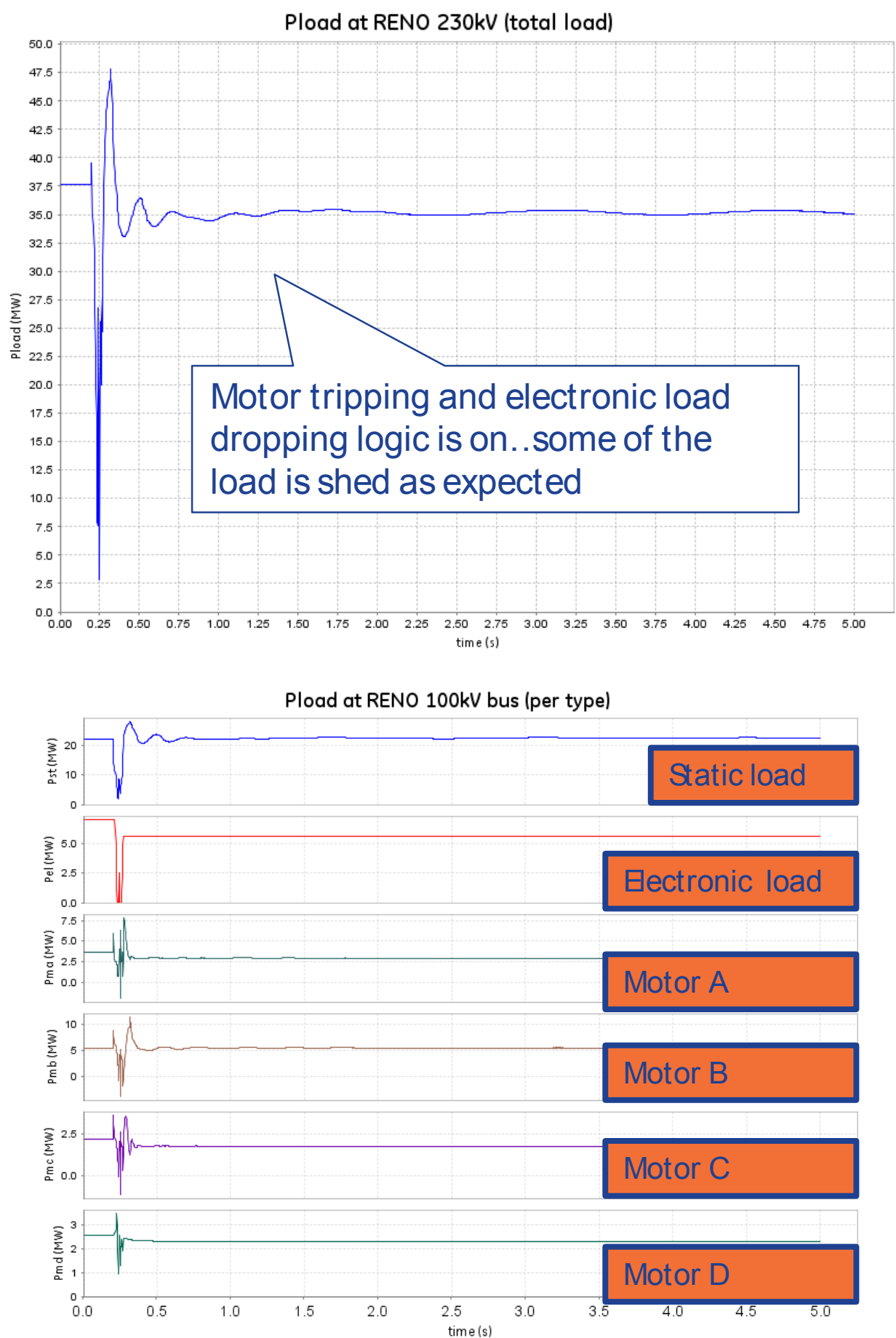

Figure 55. Details of active power composite load response with motor tripping 


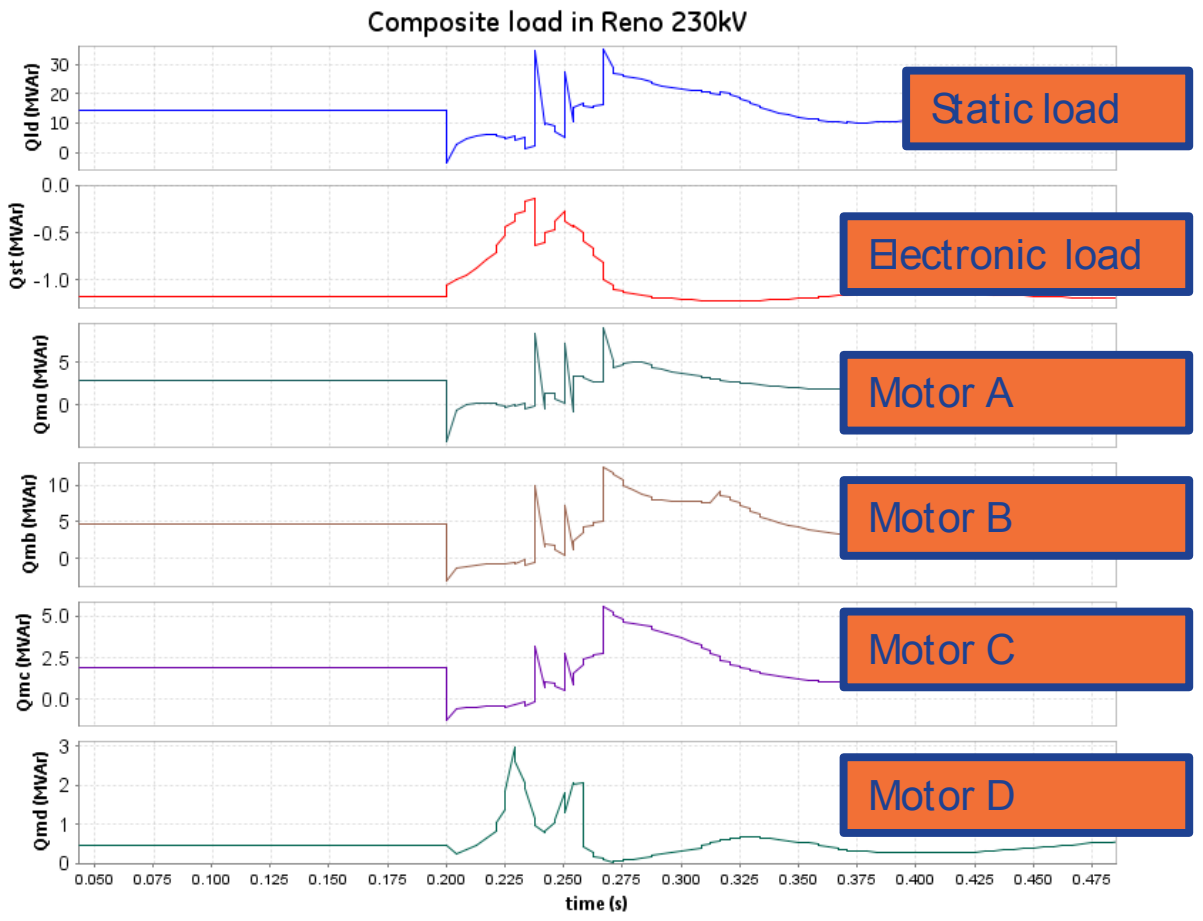

Figure 56. Details of reactive power response with motor tripping

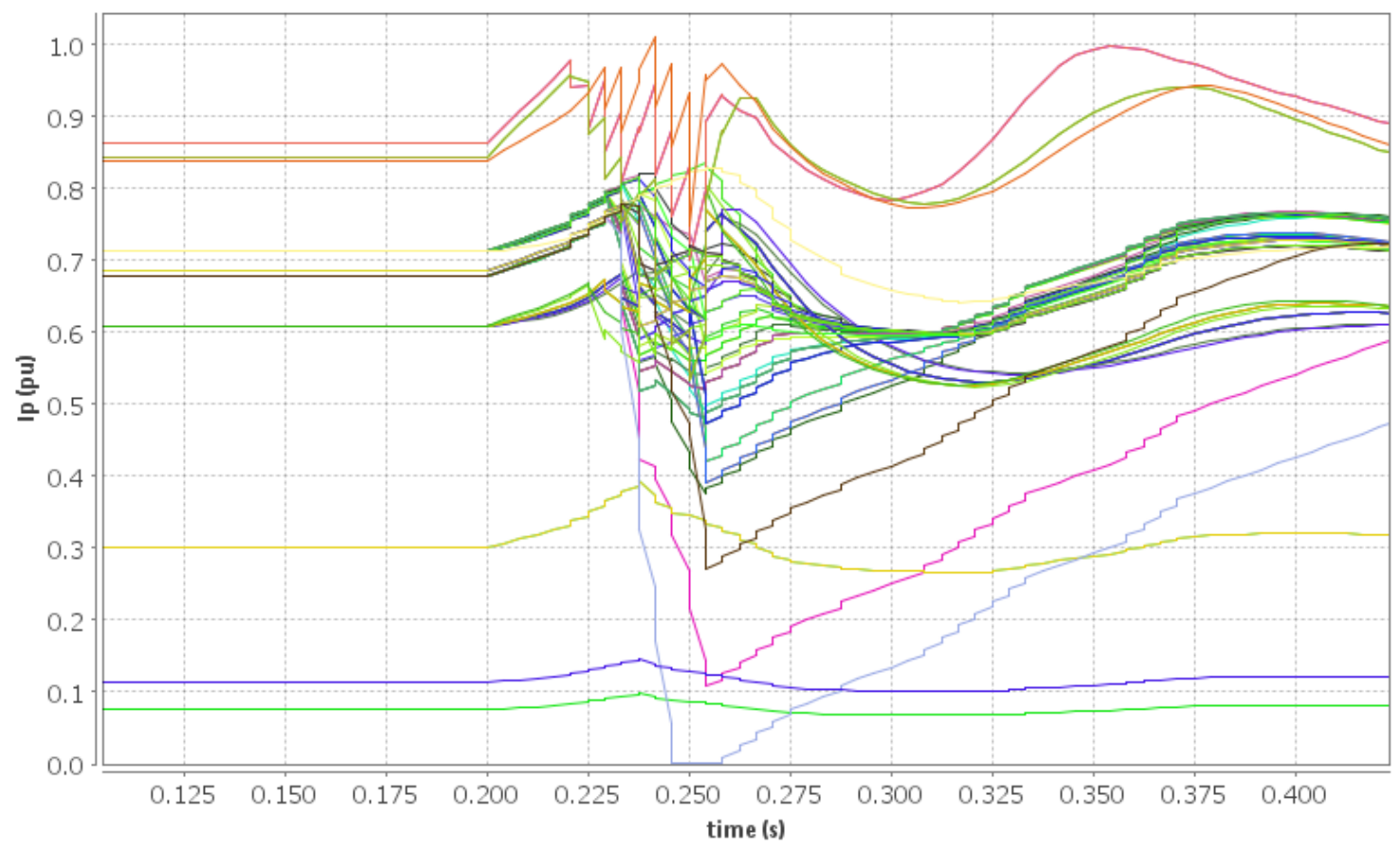

Figure 57. Active current response for WTGs in Area 73 (WAPA RM)

\subsubsection{Standard WECC Load Model}

Another sensitivity case was executed using the standard WECC load model. This combination of much simpler load elements is widely used today in simualtions of the Western 
Interconnection. Most of the load was static (i.e., so-called ZIP load), with a high degree of voltage dependence. That is, the active power consumption dropped in an algebraic relationship to the voltage at that node. Coefficents varied by location in the West, but many of the loads were close to a constant current-i.e., the active power dropped in linear proportion to the terminal votlage.

The bus voltages in Area 65 (PACE) are shown in Figure 58 for the Aeolus fault on the reduced stress (Extreme-LW) case, with the tuned LVPL settings. This case, which was stable with the compound load (see Figure 39), survived the fault but was driven into unstable oscillations. Notice that compared to the unstable cases with the compound load (e.g., Figure 23), these oscillations were much more regular and were of a rather higher frequency.

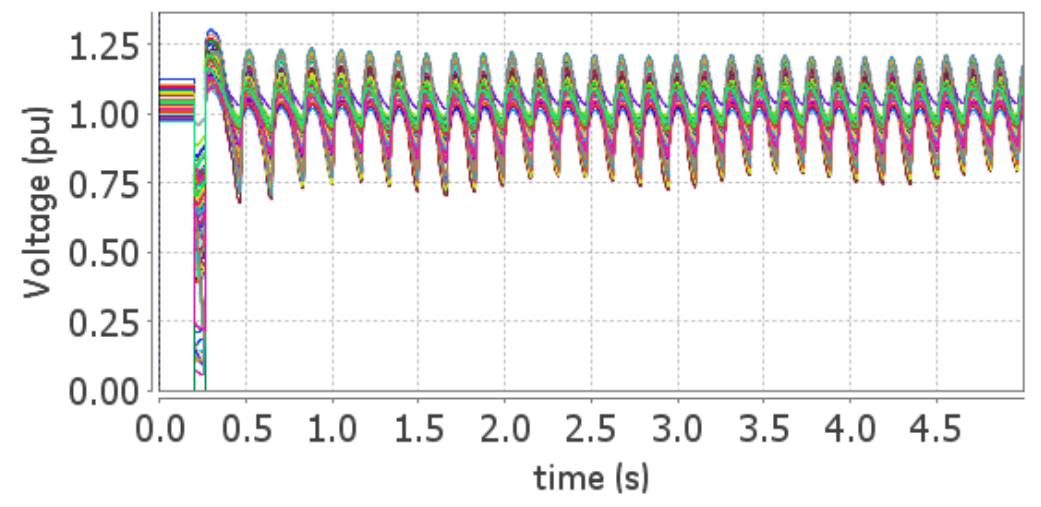

Figure 58. Reduced stress case with (old) standard WECC load model

This is an interesting result. The standard WECC load model responded rapidly and more strongly to a decline in voltage. The act of the LVPL increasing the active power after the fault resulted in dropping voltage, which depressed the load power and increased the net power exported from the system. The result was less stable. Because the oscillations were so regular and the behavior of the load was simpler, the phase-plane plot of $\mathrm{V}$ and $\mathrm{P}$, as shown in Figure 59, resulted in a clearly identifiable nose, which has been sketched in red. 


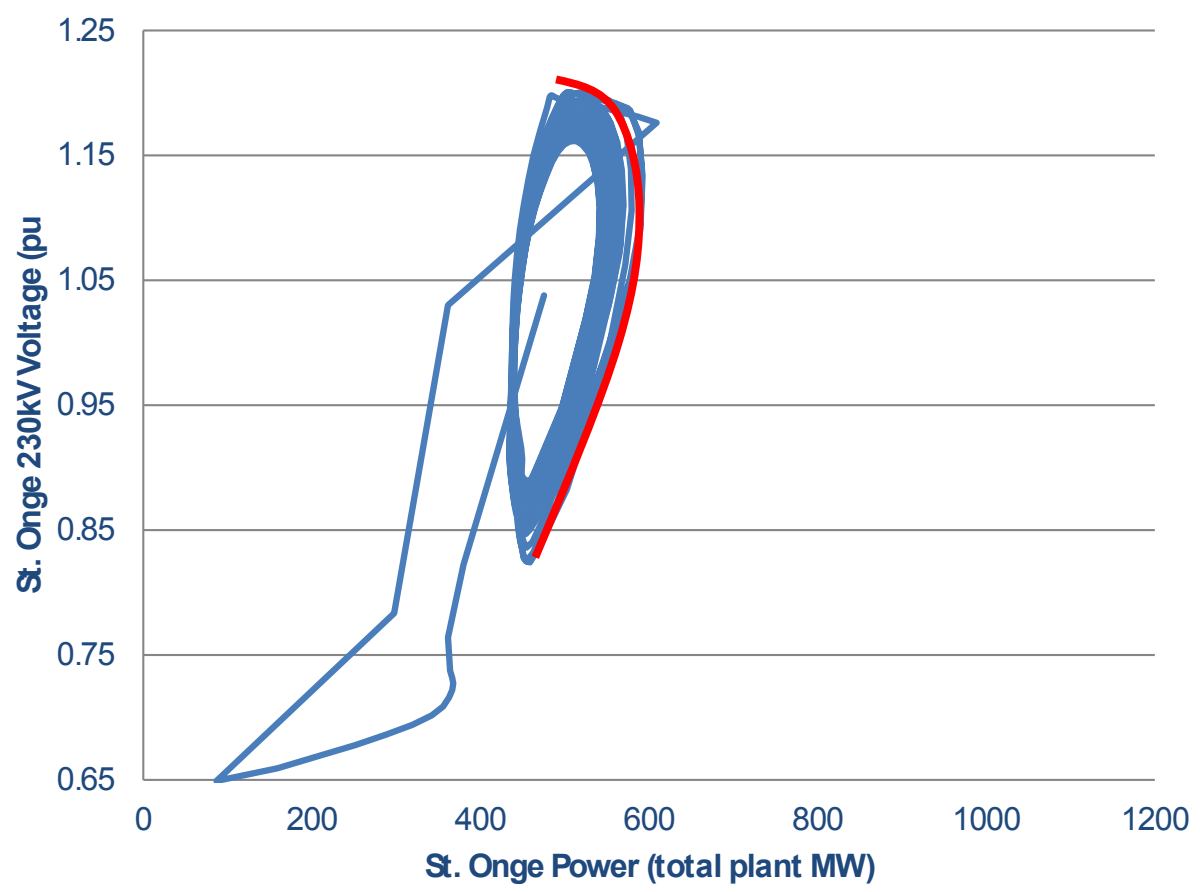

Figure 59. Dynamic PV for St. Onge wind power plant with standard WECC load model

With the standard WECC load model, the LVPL action as tuned was not enough to save the system from the Aeolus fault. However, with the OEM- (GE-) specific WTG model with the weak grid control features enabled, as shown on the right side in Figure 60, the system survived the fault and did not enter the nonlinear oscillations.
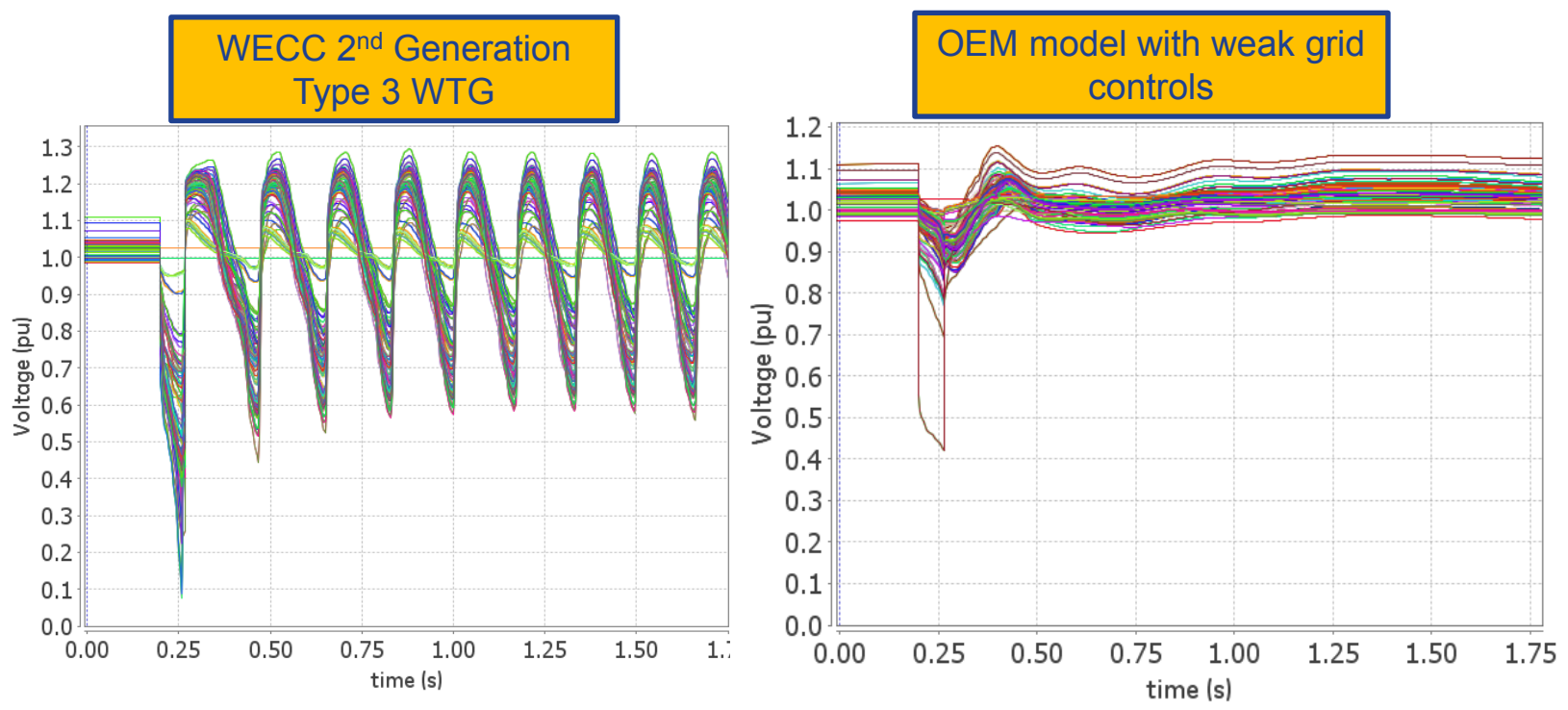

Figure 60. Different WTG functionality with standard WECC load model 


\subsection{Identifying Weak Grid/High-Renewable Nodes}

With the addition of inverter-based generation, SCR is a well-established measure of system strength relative to the rating of HVDC or other individual inverter-based resources, such as a single wind or PV site. Composite short-circuit ratio (CSCR) is a newer concept, that gives similar information for multiple inverter-based resources in electrical proximity but not exactly sharing the same point of interconnection. Inverter-based generation operating with a low SCR can have control stability issues.

For a simple system that has a single wind power plant, the SCR is defined as the ratio of shortcircuit MVA to the MW rating of the wind power plant. For a system that has multiple wind power plants, the CSCR is calculated as the short-circuit MVA seen by all the plants to the total MW rating of all the plants. The short-circuit MVA is typically calculated on the low-voltage side of the plant transformer(s). SCRs are used to identify weak grid issues for a given plant or group of nearby plants. This exercise attempts to provide a measure to identify the degree of renewable "saturation" at the transmission buses.

In simple terms, the concept is to calculate the strength, in the conventional sense, of each transmission bus, and to calculate how much inverter-based generation a bus "sees." The idea is that, from the perspective of a weak grid, a planner can focus on transmission buses that are relatively weak and that "see" a lot of renewables. The planner does not need to worry about strong buses that "see" relatively little inverter-based generation. Further, buses that are both weak and see little renewables are on the periphery and will likely have only localized issues.

The sketch in Figure 61 shows the concept for the two metrics. The short-circuit MVA (SCMVA) (on the left) is calculated from the short-circuit contribution synchronous machines, with no contribution from the wind power plants (or, more generally, the inverter-based generation). The effective renewable MVA (ERMVA) is calculated similarly for the inverterbased resources, but the calculation uses an impedance of 1.0 on the plant base. This is a reflection of the fact that these devices are, for the purpose of this screening method, perfect current sources that are limited by the device rating. In this construct, a bus that is in electrical proximity to several large plants will see a large ERMVA.

The tool allows an orderly search for locations that are strongly influenced by large wind power plants and have relatively little synchronous generation nearby. In Figure 62, the screen was applied to transmission substations ( $230 \mathrm{kV}$ and above) in Montana, Wyoming, and Colorado for the three cases. The difference between the Base case (locus of blue diamonds) and the Hi-Mix case (red squares) was because of two factors: added wind and decommitted synchronous generation. Whereas the difference between the Hi-Mix case and the Extreme case (green triangles), which had the same high renewables topology, was only because of decommiting synchronous generation. The lines are the loci of ratios between the synchronous short-circuit strength and the amount of wind experienced by a bus. This measure is similar to, but not the same as, the SCR and CSCR measures used to evaluate system strength with respect to wind power plant rating. The progression from 2:1 SCMVA to ERMVA (the blue line), to 1:1 (the black line), to 1:2 (the red line) is toward a weaker grid condition. Points near the red line experienced twice as much wind as short-circuit strength provided by the synchronous machines. 
Two buses are labeled on the plot: St. Onge and Snowy Range. They have big wind power plants nearby (high ERMVA) and relatively low SCMVA. They are near the red line, and they are big. It is of interest to note that these two buses, which fell out of this screening, in fact demonstrated some of the most interesting behavior. St. Onge was examined in this section, and the Snowy Range bus will be examined closely in the next section on HVDC. As noted in Section 2.1, the $230-\mathrm{kV}$ transmission line from Snowy Range was added to allow for evacuation of the higher power production of the Extreme case. This stiffened the bus, so unlike most other buses in the system, Snowy Range actually got better between the Hi-Mix case and the Extreme case. This is confirmation of the expected behavior for the method, and it illustrates a useful aspect of it.

Figure 63 shows the ratio of SCMVA to ERMVA sorted from lowest to highest in Area 73 (WAPA RM). Again, this screening shows buses that proved to be highly influenced by the wind generation. Finally, Table 4 shows a listing of the two quantities for the top buses in the region. The ratio of the metrics, labeled as CSCR (for compound short circuit ratio), is not the same as traditional SCR. For example, the short-circuit strength of the St. Onge $230-\mathrm{kV}$ bus is 1,700 MVA. The wind power plant added there is of 1,200 -MW rating, so by some traditional approach, the SCR would be $1.7 / 1.2=1.4$, a level that is understood to be potentially problematic for inverter-based generation.

Although these metrics were useful in identifying locations that were vulnerable, it is likely that further refinement would make them more valuable. Some of the same challenges to creating meaningful PV and PQ curves apply here, and the development of these types of screening tools should proceed together with other dynamic voltage stability analysis techniques.

SCMVA

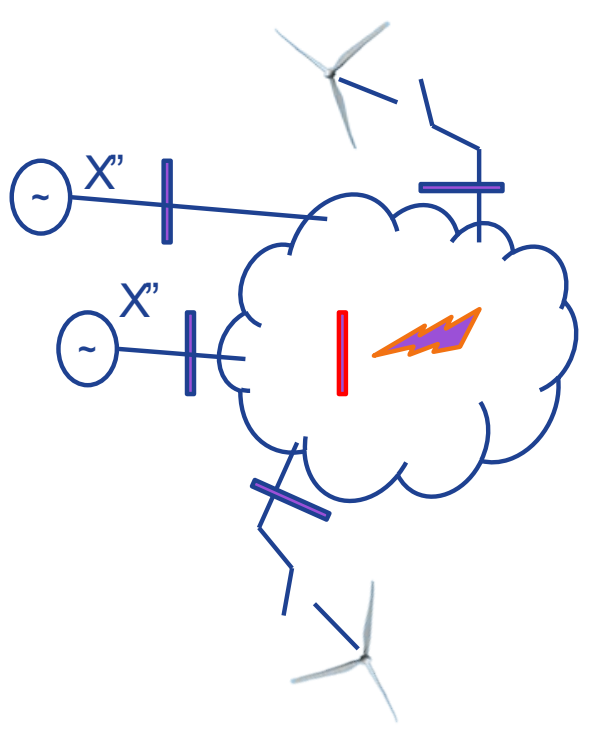

ERMVA

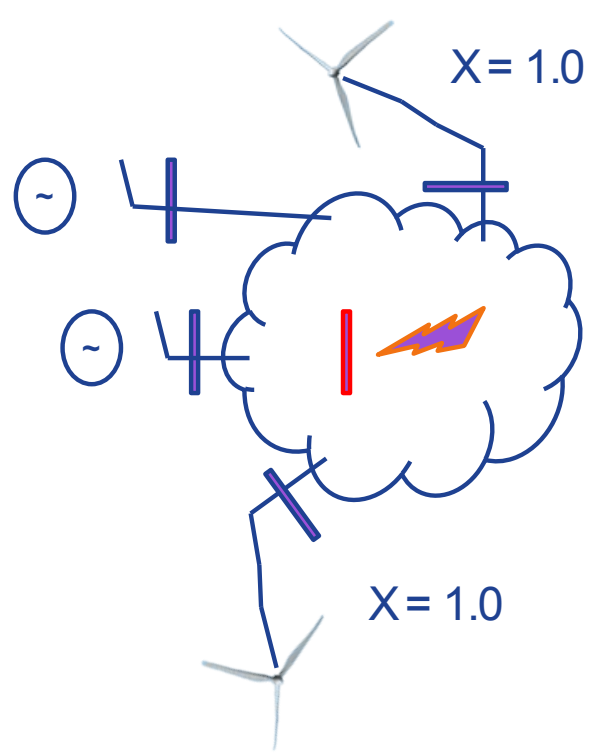

Figure 61. SCMVA and ERMVA 
Ratio of Short Circuit MVA to Effective MVA of Renewables at all $230 \mathrm{kV}$ and $345 \mathrm{kV}$ Buses in Montana, Wyoming and Colorado

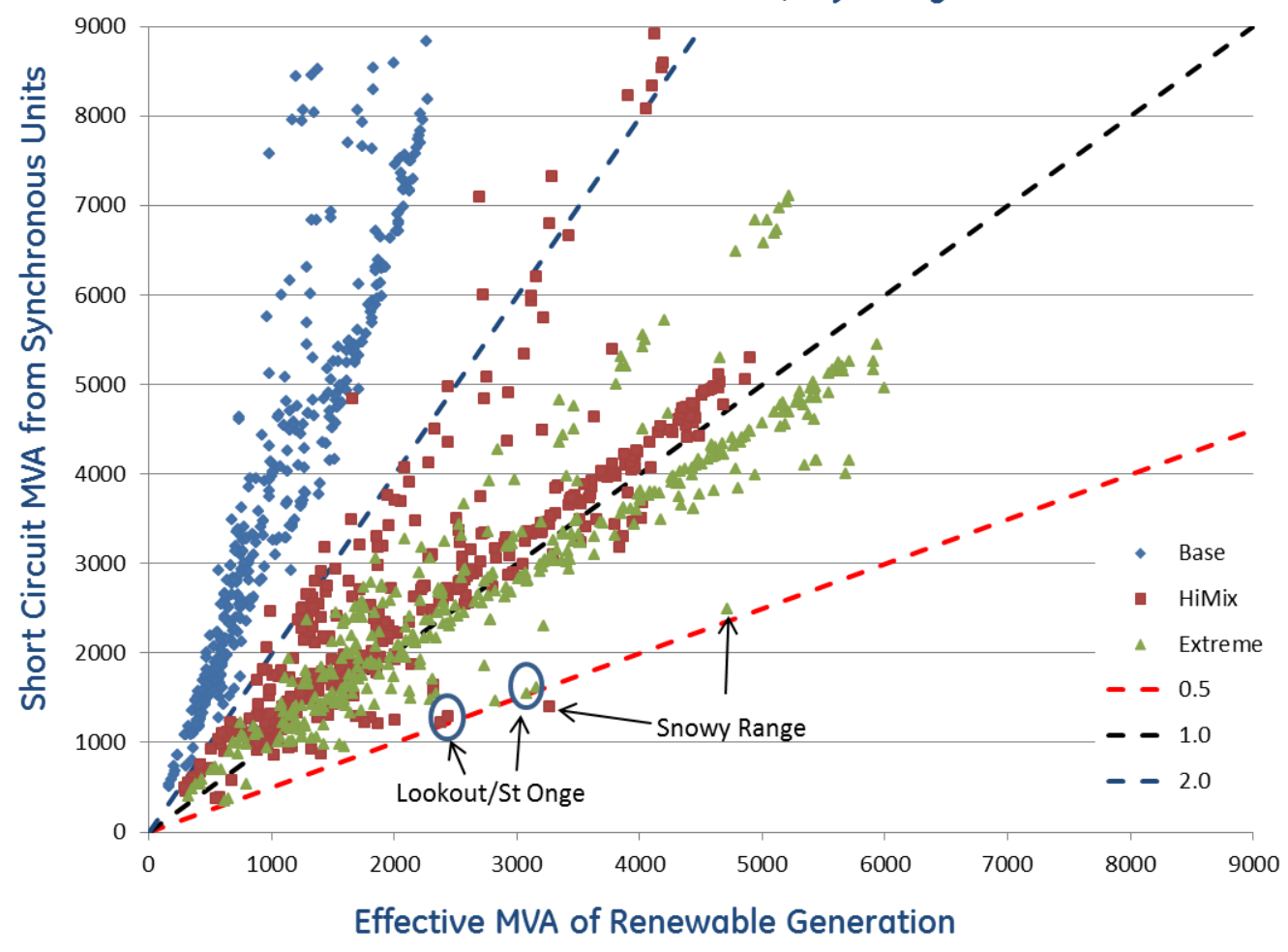

Figure 62. ERMVA of renewables compared to synchronous SCMVA 


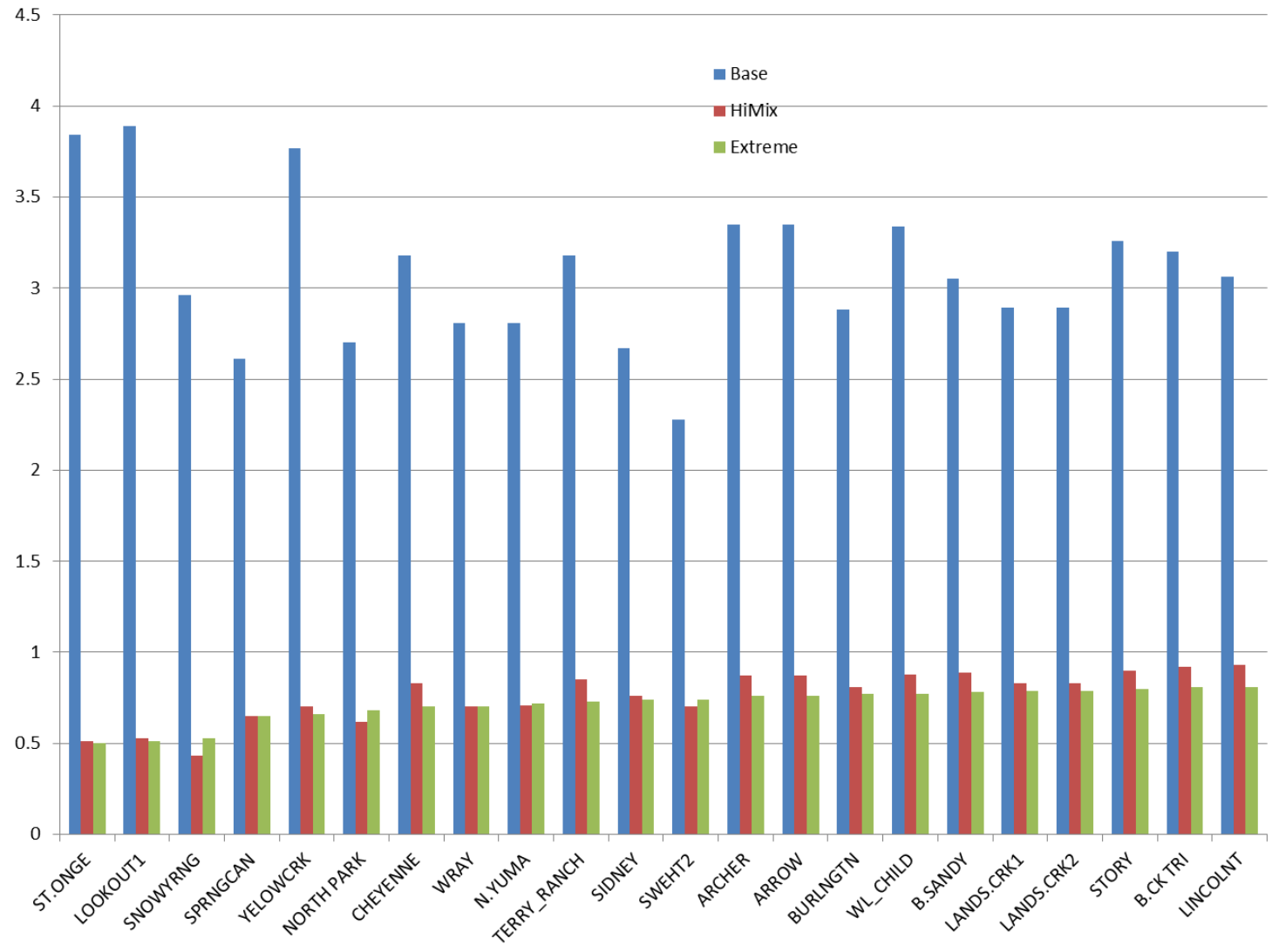

Figure 63. SCMVA/ERMVA for most saturated Area 73 (WAPA RM) buses 
Table 4. Top 10 Wind-Saturated Buses

\begin{tabular}{llllllllllll}
\hline Area & Name & $\mathbf{k V}$ & $\begin{array}{l}\text { Base } \\
\text { SCMVA }\end{array}$ & $\begin{array}{l}\text { Base } \\
\text { ERMVA }\end{array}$ & $\begin{array}{l}\text { Base } \\
\text { CSCR }\end{array}$ & $\begin{array}{l}\text { HiMix } \\
\text { SCMVA }\end{array}$ & $\begin{array}{l}\text { HiMix } \\
\text { ERMVA }\end{array}$ & $\begin{array}{l}\text { HiMix } \\
\text { CSCR }\end{array}$ & $\begin{array}{l}\text { Extreme } \\
\text { SCMVA }\end{array}$ & $\begin{array}{l}\text { Extreme } \\
\text { ERMVA }\end{array}$ & $\begin{array}{l}\text { Extreme } \\
\text { CSCR }\end{array}$ \\
\hline 73 & SNOWYRNG & 230 & 1744 & 589 & 2.96 & 1394 & 3268 & 0.43 & 2489 & 4712 & 0.53 \\
73 & ST.ONGE & 230 & 1700 & 443 & 3.84 & 1228 & 2384 & 0.51 & 1540 & 3085 & 0.50 \\
73 & LOOKOUT1 & 230 & 1816 & 467 & 3.89 & 1284 & 2445 & 0.53 & 1620 & 3158 & 0.51 \\
70 & PTZLOGN & 230 & 1125 & 960 & 1.17 & 876 & 1405 & 0.62 & 955 & 1596 & 0.60 \\
73 & NORTH & & & & & & & & & & \\
73 & PARK & 230 & 1613 & 598 & 2.70 & 1252 & 2008 & 0.62 & 1853 & 2740 & 0.68 \\
62 & SPRNGCAN & 230 & 1508 & 577 & 2.61 & 1215 & 1870 & 0.65 & 1484 & 2293 & 0.65 \\
70 & WILSALL & 230 & 1673 & 580 & 2.89 & 1568 & 2322 & 0.68 & 1472 & 2821 & 0.52 \\
70 & CO_GRN & 230 & 532 & 349 & 1.52 & 390 & 577 & 0.68 & 368 & 650 & 0.57 \\
70 & TWNBUTTE & 230 & 505 & 335 & 1.51 & 371 & 548 & 0.68 & 351 & 615 & 0.57 \\
\hline
\end{tabular}

65 


\subsection{Discussion: Stress, Functionality, and Performance}

The sequence of cases presented in this section illuminates the issue of high penetrations of wind (and solar, and by proxy other inverter-based generation) when there is little synchronous generation running within a large geographic area.

The details of the problems around St. Onge are solely a construct of this study: the authors have no knowledge of proposals to build a large wind power plant specifically at that site. In this study, the region is host to multiple large wind power plants because the wind resource is excellent. There are many plants in the area, and many more could be built. But the exact locations, size, and transmission topology will undoubtedly be substantially different. However, the cases show some fundamental relationships between stress on the grid, the performance characteristics of the wind power plants, and the performance of transient stability.

A visualization of this relationship is shown in Figure 64. The general idea is that as wind power plant functionality for weak grids is provided, transient stability will improve. Further, as system stress increases, transient stability will degrade. Some of the constituents of stress and weak-grid functionality are listed in the figure annotations. Other constituents could be added.

For example:

- High line loading

- Low SCR

- Limited dynamic reactive

- Limited lines/transformations

Poor Performancel

Unstable

\section{Stress}

For example:

- Well managed line loading

- Higher SCR

- Adequate dynamic reactive capability

- Adequate lines/transformations
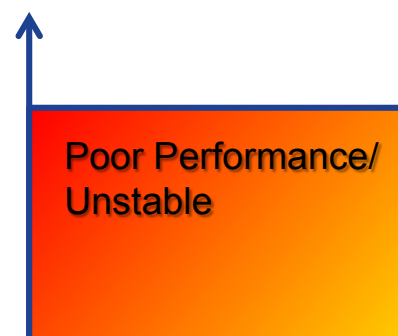
To put this generic visualization in the context of the results presented above, six of the cases described above were placed in this stress-functionality space in Figure 65. The stress is reflected by the two Extreme cases along the Y-axis, and the functionality is related to the LVPL, with the highest functionality coming from the OEM-specific model with weak-grid (grid-dependent power limits) features enabled. The Hi-Mix condition, had it been included in this figure, would be lower. Recall that the "default" functionality was sufficient to give satisfactory (i.e., "green") performance in that scenario.

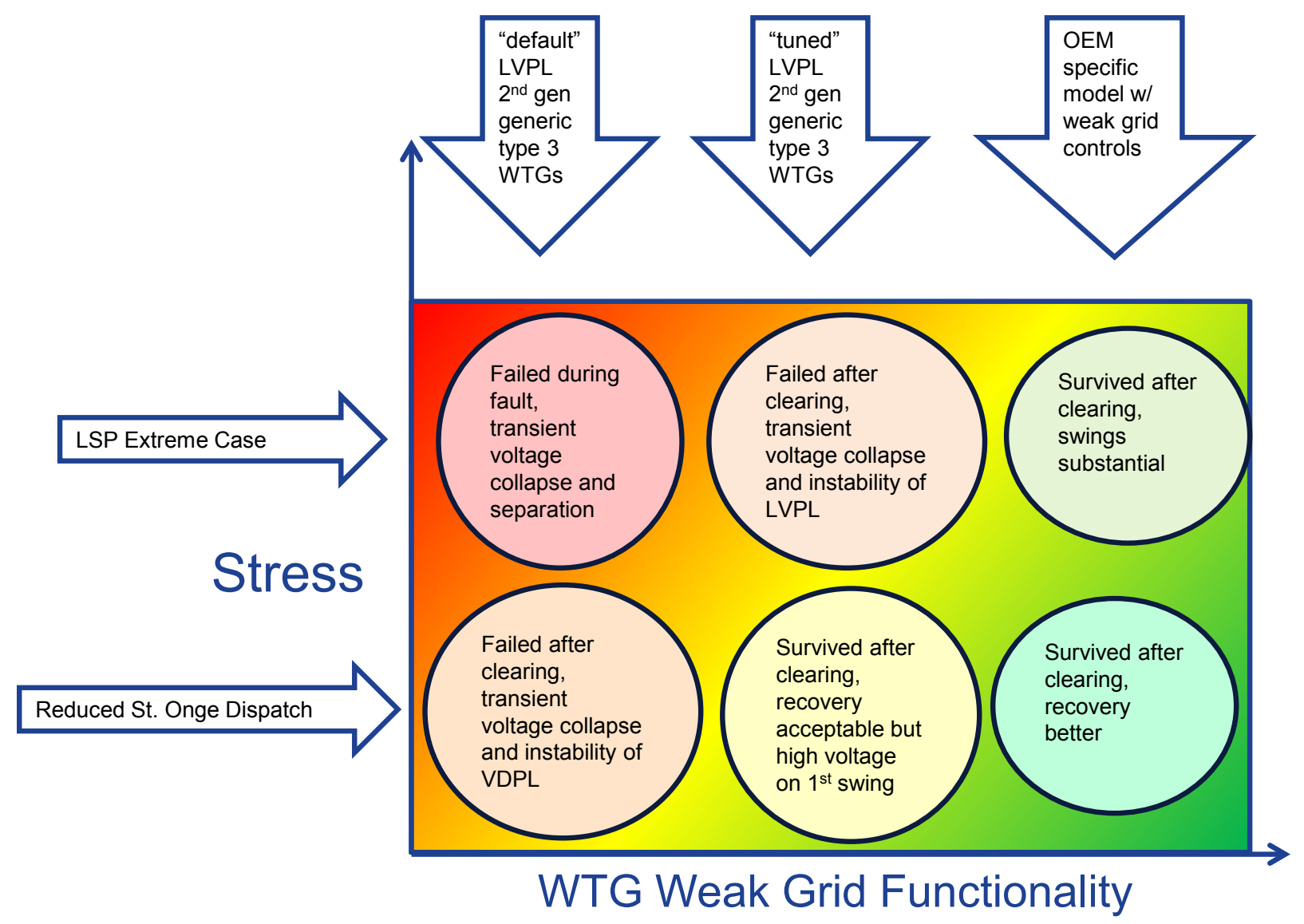

Figure 65. Visualization for results around St. Onge

It is important to keep the stress space of this visualization in context. In Figure 66, the annual generation penetration duration curves for all of WECC are shown again, with the instantaneous penetrations of wind and solar for the Hi-Mix and the Extreme cases noted. The Extreme case was from the top $1 / 2$ of $1 \%$ of penetration observed in the year. That point, which had an unreasonably large wind power plant on the extreme periphery of the Western Interconnection, the output of which was overloading the transmission providing evacuation, had stability problems when the wind power plants had limited functionality directed at giving good weak grid performance. When the dispatch of that single plant was reduced by $300 \mathrm{MW}$, from a total of 57,600 MW in the U.S. Western Interconnection, the performance improved substantially. The point is that the details of stress and wind power plant functionality matter most when the system is pushed to the extreme. 


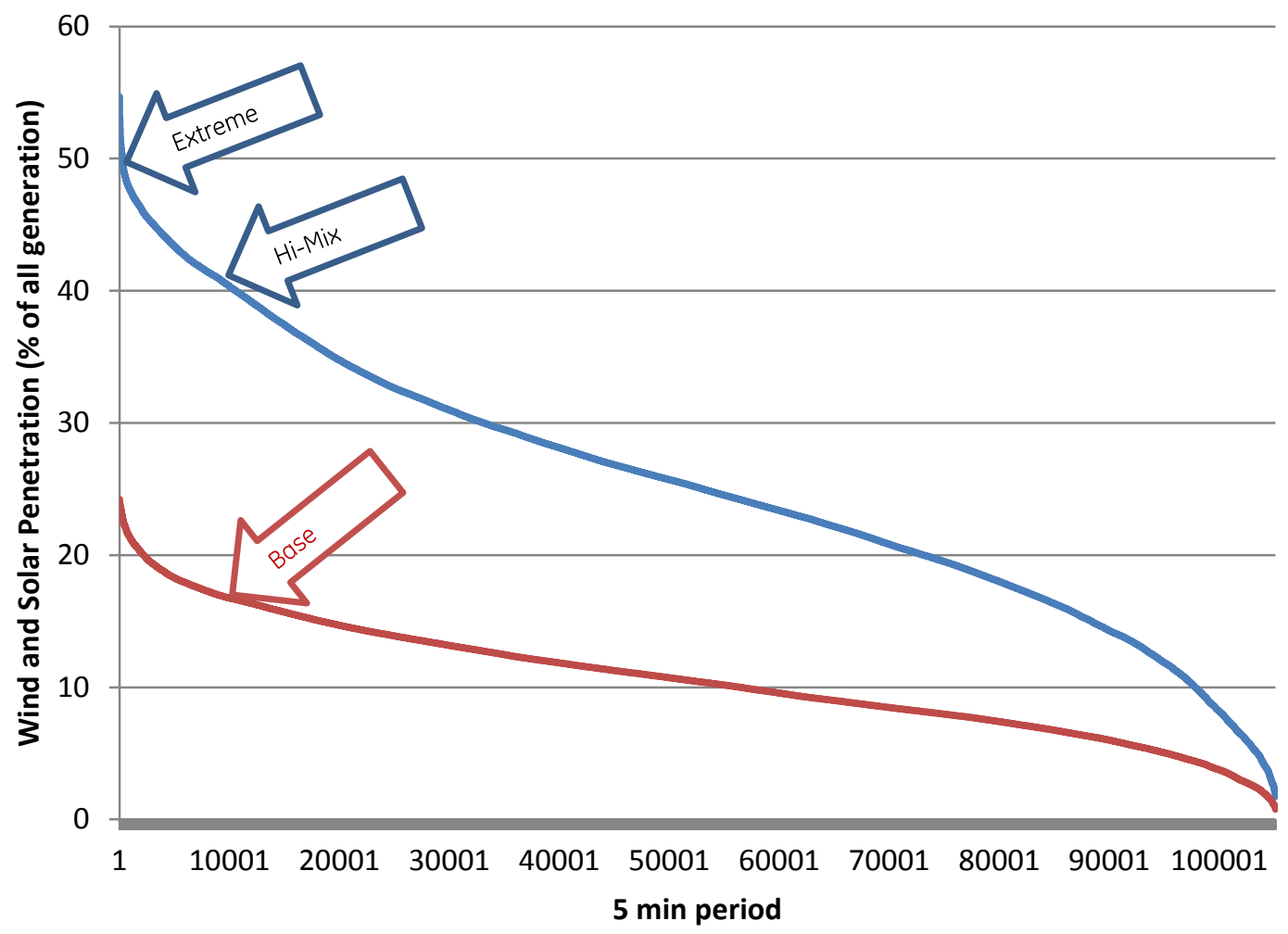

Figure 66. Penetration duration curves from WWSIS-2 production simulations

\subsection{Exploration of HVDC Ties into High Wind Region}

The construction of large HVDC ties electrically connecting wind-rich regions to major load centers has long being considered a potential solution to move large amounts of wind energy from remote locations to where it is needed most. In fact, most of the regions that have abundant wind potential in the Western Interconnection are located relatively far away from large consuming centers. Therefore, this study explored how HVDC lines will perform when they serve as major corridors for wind-generated power.

This chapter examines the impact of a new, hypothetical HVDC link connecting eastern Wyoming, a region that has significant wind potential, and the Las Vegas area, which is predominantly a load center. A pictorial representation of the HVDC link is given in Figure 67. To maintain a similar power transfer capability, Gateway South was disconnected and replaced with the HVDC link. In addition to that, the HVDC link was rated 3,000 MW, which is at or near the maximum rating that present technology allows for 500-kV DC bi-poles. This gives a similar MW-transfer capability to the Gateway South transmission corridor.

The addition of the HVDC link must be evaluated like any other significant topological modification in the system. If an HVDC link carrying 3,000 MW is suddenly blocked (because of a fault or an unintended trip), system-wide stability implications must be studied and well understood. For that purpose, this chapter analyzes several scenarios in which the block of the HVDC link was considered as a single event (N-1) or as part of a more severe event (N-1-1). For simplicity, the loss of both HVDC poles was considered an $\mathrm{N}-1$ contingency, even though the 
North American Electric Reliability Corporation standard TPL-001-1 considers it an N-2 event (Category C contingency). In addition to losing the HVDC, the concurrent loss of Gateway West was also be considered in a hypothetical N-1-1 scenario. The details of each scenario as well as remedial actions considered to avoid loss of transient stability are discussed below.

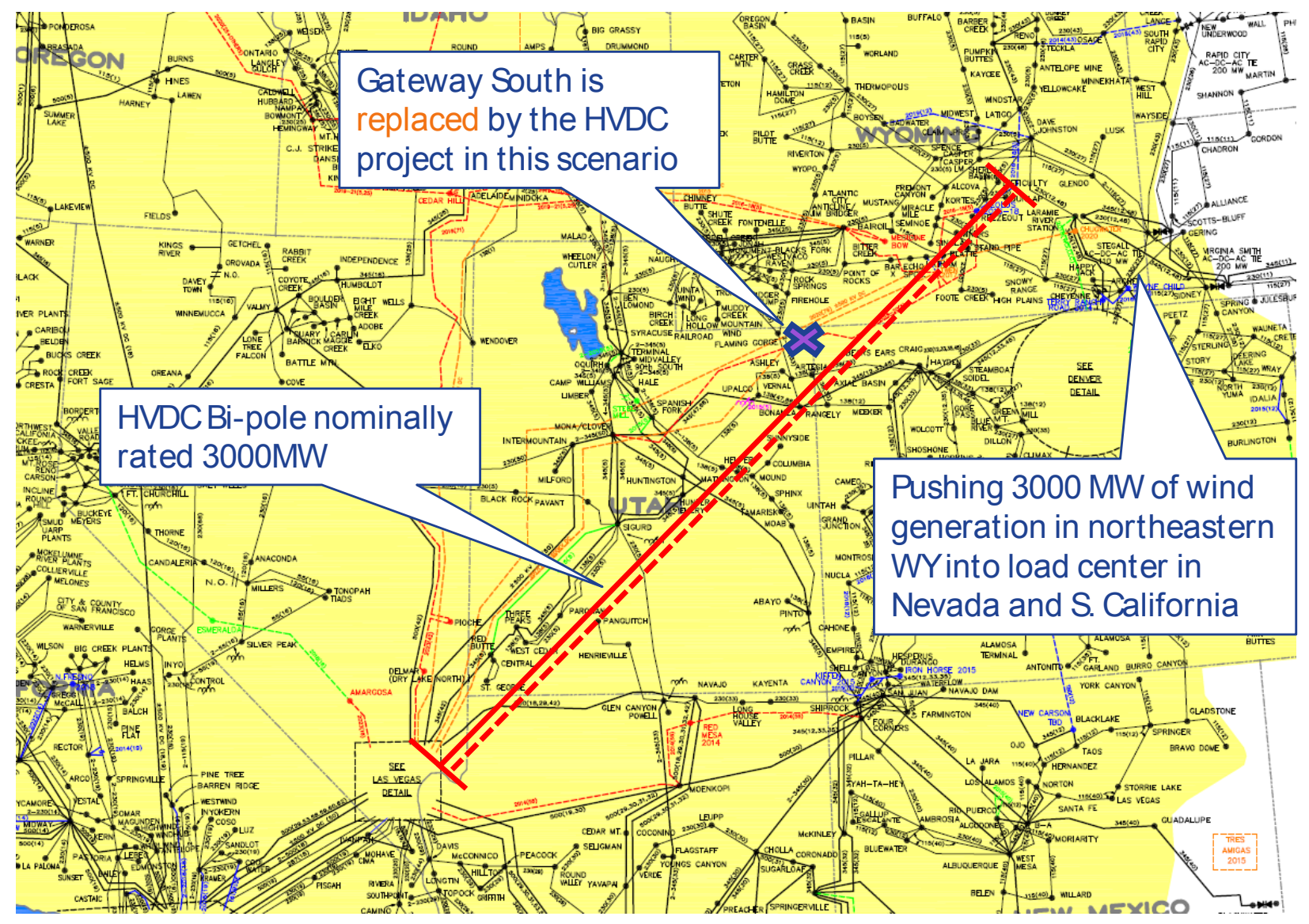

Figure 67. Addition of HVDC link between Aeolus (WY) and McCullough (NV)

\subsection{HVDC Model}

Accurate modeling of HVDC links for specific system planning studies is complex. Projects are tailored to specific requirements of the application for which they are built. Each manufacturer of HVDC has its own control and design philosophy, so accurate and generic modeling of HVDC is an oxymoron. Because this study is not focused at the behavior of the HVDC link but rather system response to the loss of the link, a simple model was used to represent the HVDC system. This approach allowed for a high-level view of the systemic impacts of HVDC export from a wind-rich region without the unnecessary distraction of attempting to build a detailed HVDC tie.

\subsubsection{HVDC Technology}

For bulk power transfer by DC, two basic technologies are available:

- Line commutated converter (LCC) DC, also known as "conventional HVDC"

- Voltage source converter (VSC) DC, also known as "self-commutated DC" 
LCC HVDC is well-established technology, and it is used in two major, older DC ties in the Western Interconnection: the Pacific DC Intertie and Intermountain. LCC has some fundamental characteristics that are important for this investigation:

- The converters always consume reactive power as a function of the active power they are carrying and the $\mathrm{AC}$ grid conditions.

- Reactive compensation at or near the terminal is required. This is usually in the form of shunt capacitors and filters, but it can include dynamic devices as well.

- The converters require an AC voltage with a minimum strength—i.e., short-circuit ratio- to operate.

- LCC converters cannot provide black-start capability or operate in an island with no sources.

VSC HVDC is newer technology, but it is in commercial operation in the West at the Transbay project. Again, some characteristics of VSC are fundamentally different from LCC and are important for this investigation:

- The converters have independent dynamic control of reactive power, and within rating limits they can supply the reactive power needs of the host AC system.

- Converters can operate into low or zero SCR systems when designed to do so.

- Converters can provide dynamic reactive power to the $\mathrm{AC}$ grid when the DC power is zero, if designed to do so. Depending on the reason for DC blocking, the rectifying converter may be able to continue to support the host AC system.

With these objectives in mind, the HVDC model considered in this study was a simple load sink on the rectifier end (near the Aeolus bus) and a negative load at the inverter end (near the McCullough bus). These equivalent loads were at unity power factor, representing either VSC operation or the compensating effect of shunt devices supplying the reactive power needs of the LCC converter. This simple model considered that most of the wind energy produced in eastern Wyoming would be feeding the positive load added at Aeolus. The negative load added in the Las Vegas area represented the delivery of that wind power. A pictorial representation of both the rectifier and inverter ends is given in Figure 68.

Additionally, a 500-MVA rated synchronous condenser and a 100-MVAr shunt bank was added near the rectifier end to support voltages and, in the case of the LCC, to ensure adequate shortcircuit strength for the rectifier. A representation of this additional synchronous condenser at the Aeolus 230-kV bus is provided in Figure 72. Finally, as noted in Section 2.1, and again in Section 3.10, two 230-kV circuits added for this study from Snowy Range to Cheyenne were rerouted to connect Snowy Range to Aeolus. This conformed to the expectation that large amounts of wind power from that area would want to find egress on the HVDC. Nearly 1,600 MW were transferred from Snowy Range to the Aeolus $230 \mathrm{kV}$. 

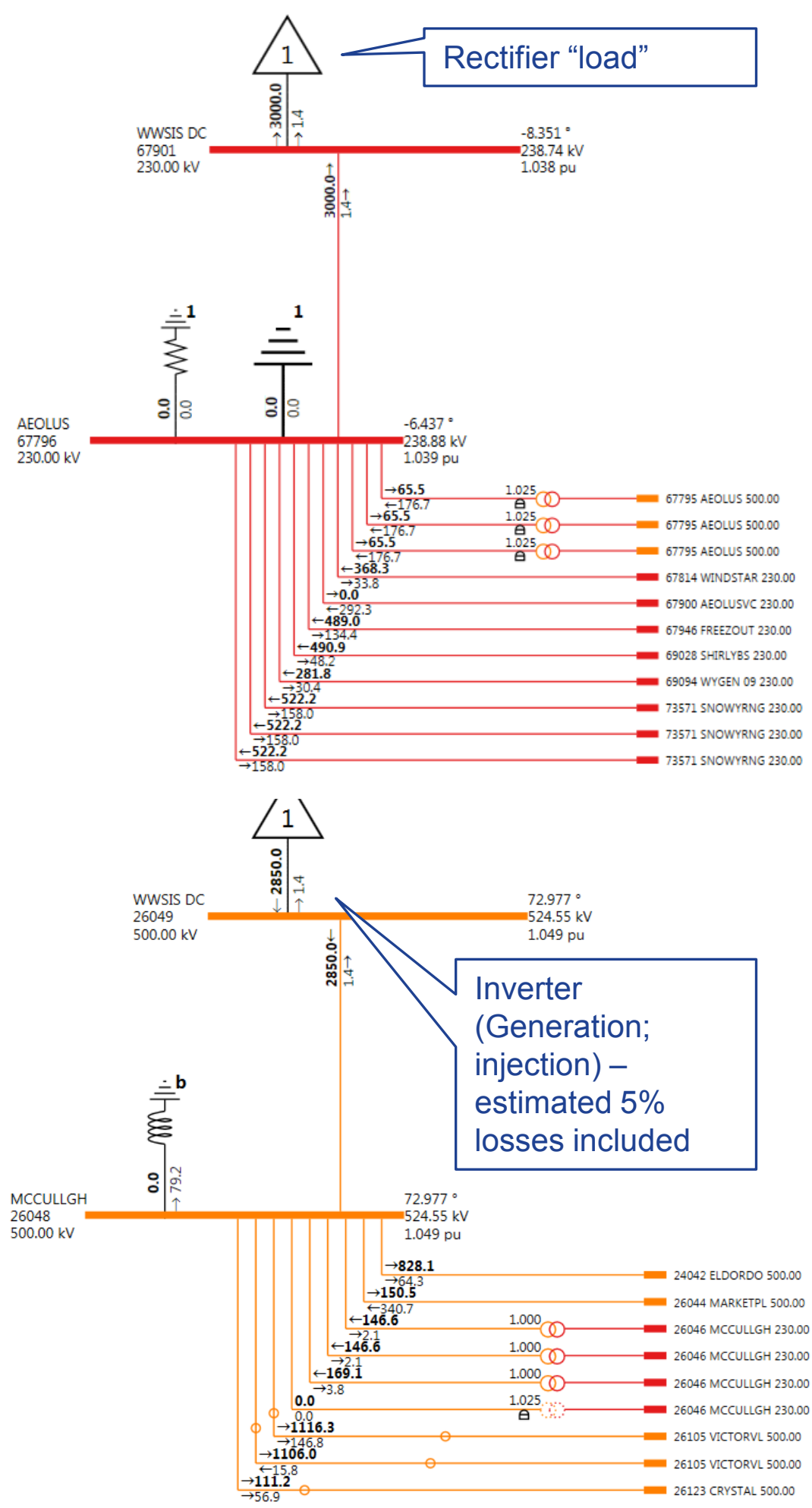

Figure 68. HVDC rectifier and inverter terminals 


\subsection{HVDC Scenarios}

\subsubsection{Block HVDC Bi-Pole (N-2)}

In this scenario, the HVDC was assumed to be carrying 3,000 MW and operating at its maximum rating. Gateway South was replaced by the HVDC line, and Gateway West was carrying nearly $200 \mathrm{MW}$.

The block of the HVDC link was done by tripping both the sink and source loads added to the Aeolus and McCullough buses. Figure 69 shows the post-contingency steady-state condition after the trip of the HVDC link. It also shows that Gateway South was offline and that the postcontingency flow on Gateway West was now approximately 2,000 MW.

Voltages on buses rated $230 \mathrm{kV}$ and above in PACE (Area 65) are shown in Figure 70 and were relatively unaltered. The only exception was Bus 67811 ANPOPC\&1 $500 \mathrm{kV}$, which settled at nearly 1.15 p.u. because of series compensation of Gateway West near that bus section and the increase in flow on the line. This overvoltage condition was corrected with the addition of 300 MVAr of shunt compensation at that bus, with the final steady-state condition being near 1.08 p.u. This corrected steady-state solution was used as the starting point of most scenarios studied in this chapter.

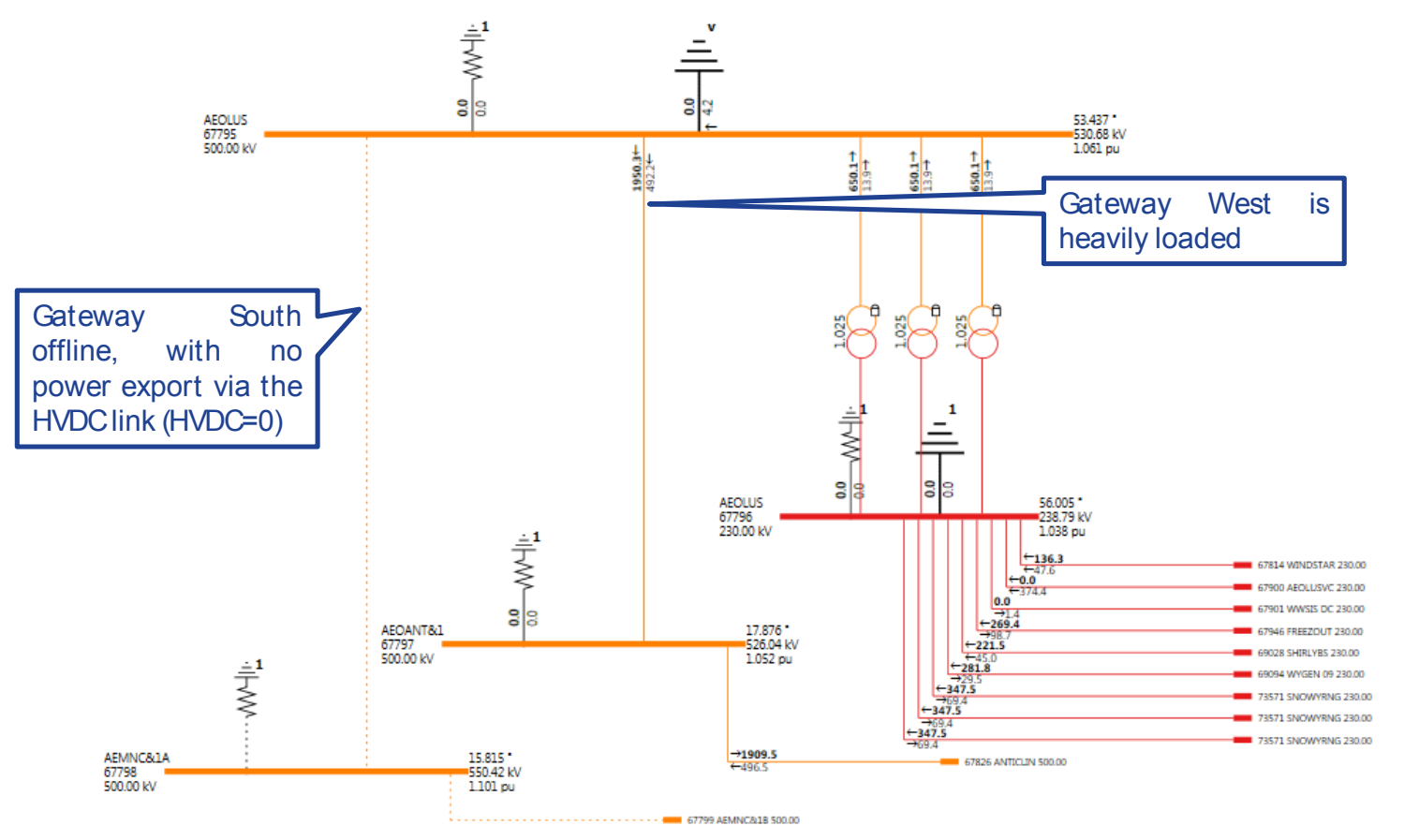

Figure 69. HVDC bi-pole block 


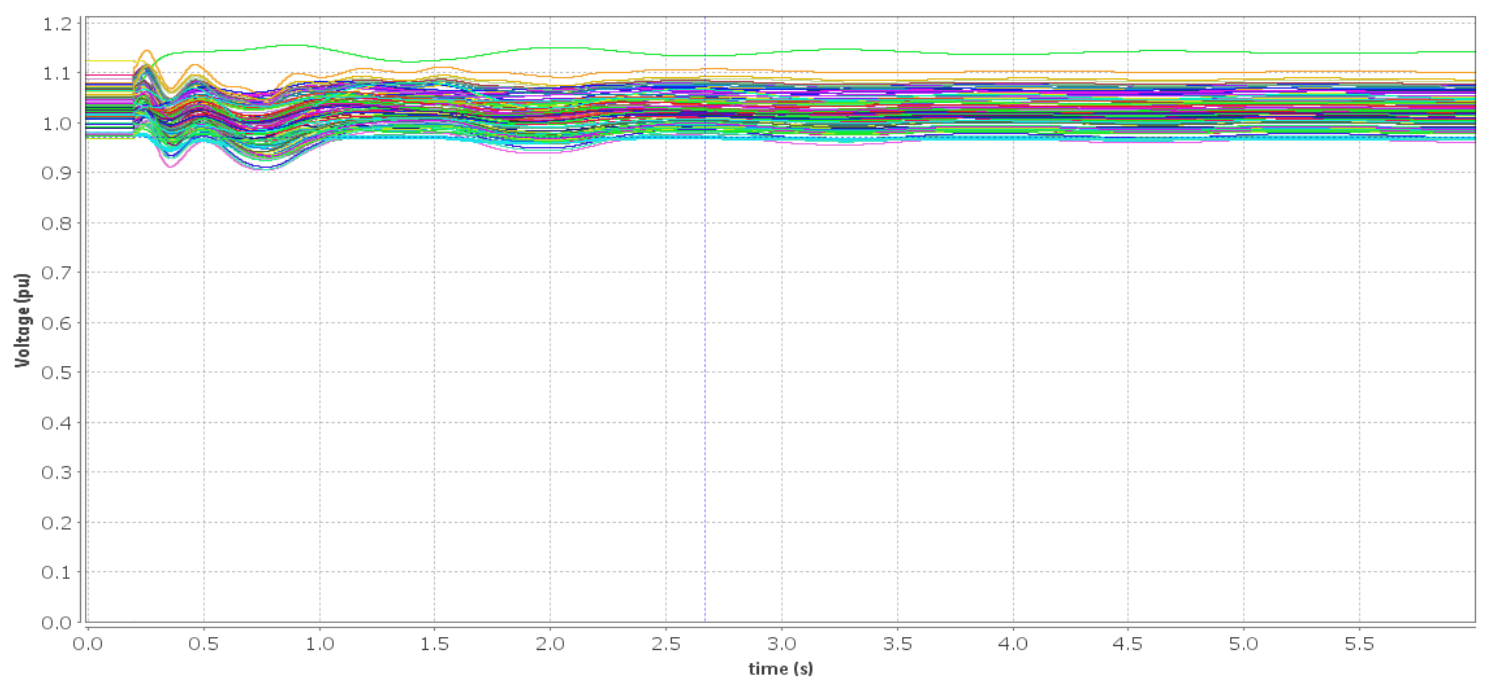

Figure 70. Area 65 (PACE) bus voltage dynamics for the HVDC bi-pole block

Overall system response was stable and relatively well damped as Gateway West served as an alternate route for most of the 3,000 MW blocked at the HVDC link. The effect of HVDC blocking on reactive power generation from the wind power plants and synchronous machines in Area 65 (PACE) is shown in Figure 71. Most machines experienced a relatively minor increase in reactive power generation, with the exception of the synchronous condenser (green trace) added to Aeolus $230 \mathrm{kV}$, which experienced a more substantial increase of reactive power production in response to increased flows through Gateway West. 

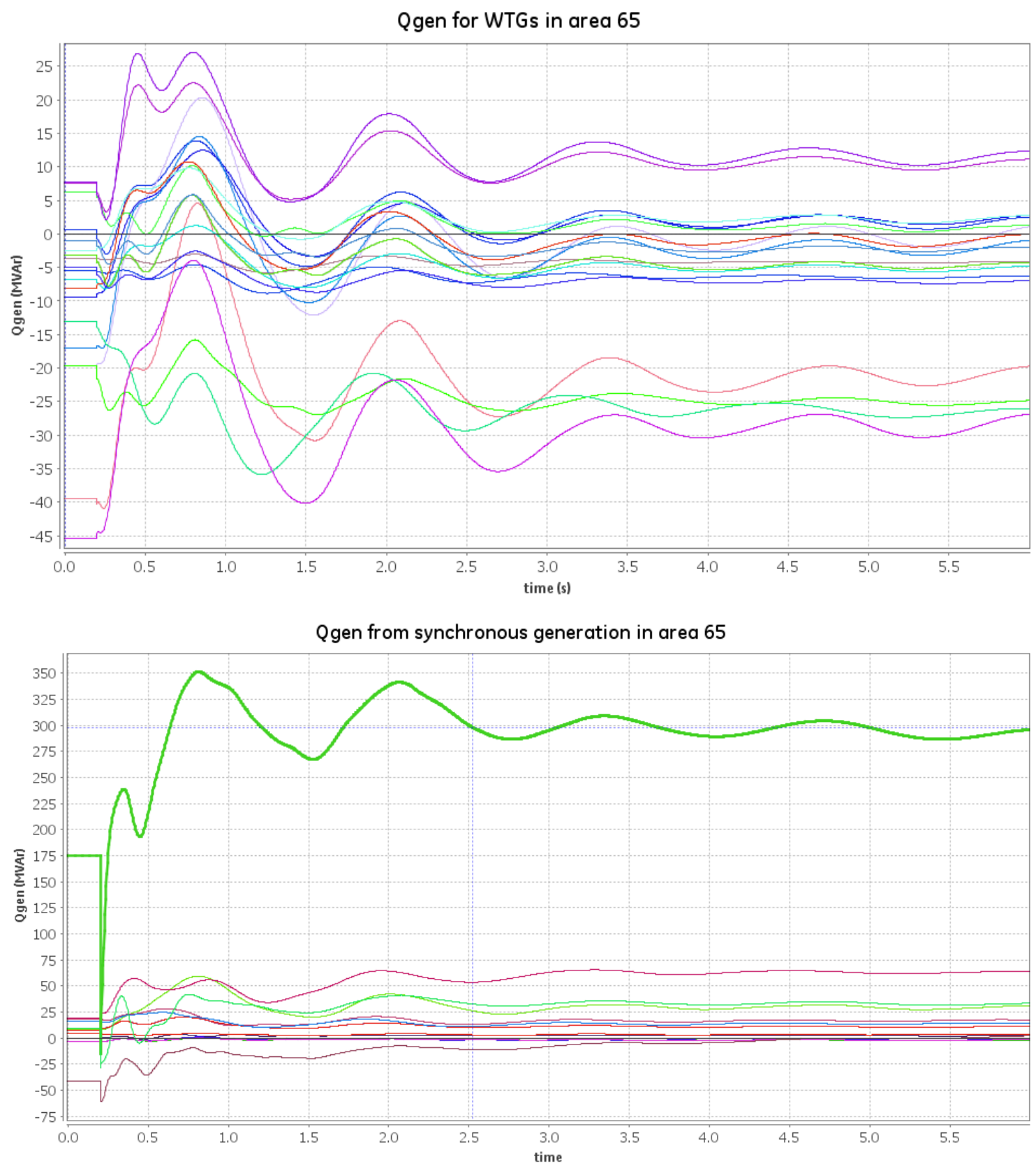

Figure 71. Effect of HVDC block on reactive power generation in Area 65 (PACE) 


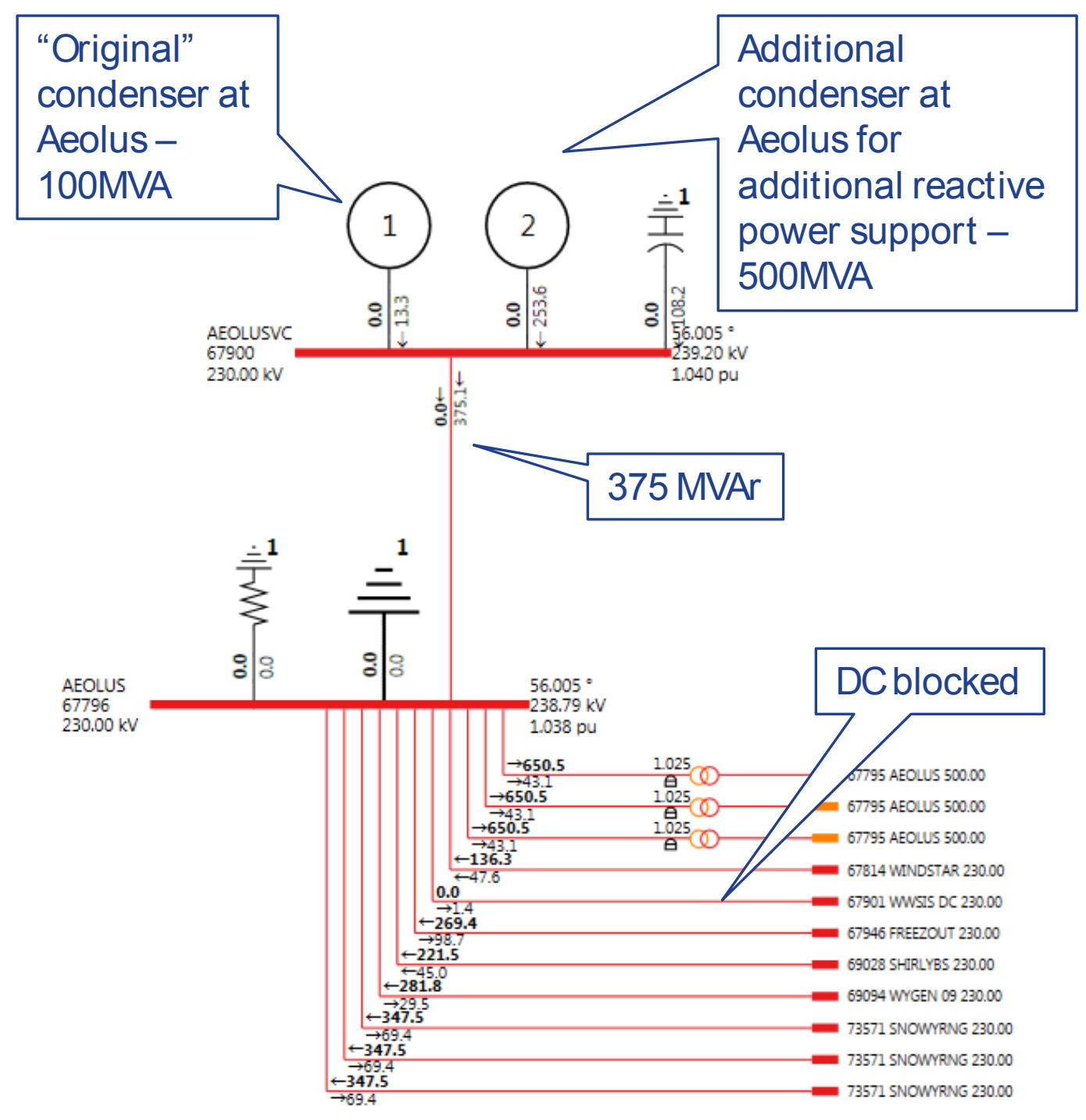

Figure 72. Blocked inverter-reactive power details

\subsubsection{N-1-1 with No Repositioning or Remedial Action Scheme}

After blocking the HVDC link and creating an N-1 condition, Gateway West was tripped to create an N-1-1 scenario. To that purpose, Gateway West was tripped at $0.2 \mathrm{~s}$ while carrying approximately 2,000 MW. An additional loss of Gateway West after the HVDC bi-pole block was a severe contingency because it eliminated two major transmission corridors for the wind power generated in the region.

Figure 73 shows all voltages in the PACE area and indicated that the system collapsed approximately $160 \mathrm{~ms}$ after the trip of Gateway West. The figure also shows that the high voltage on 67811 ANPOPC\& $1500 \mathrm{kV}$ had not been corrected yet. That correction was in place in subsequent cases. 


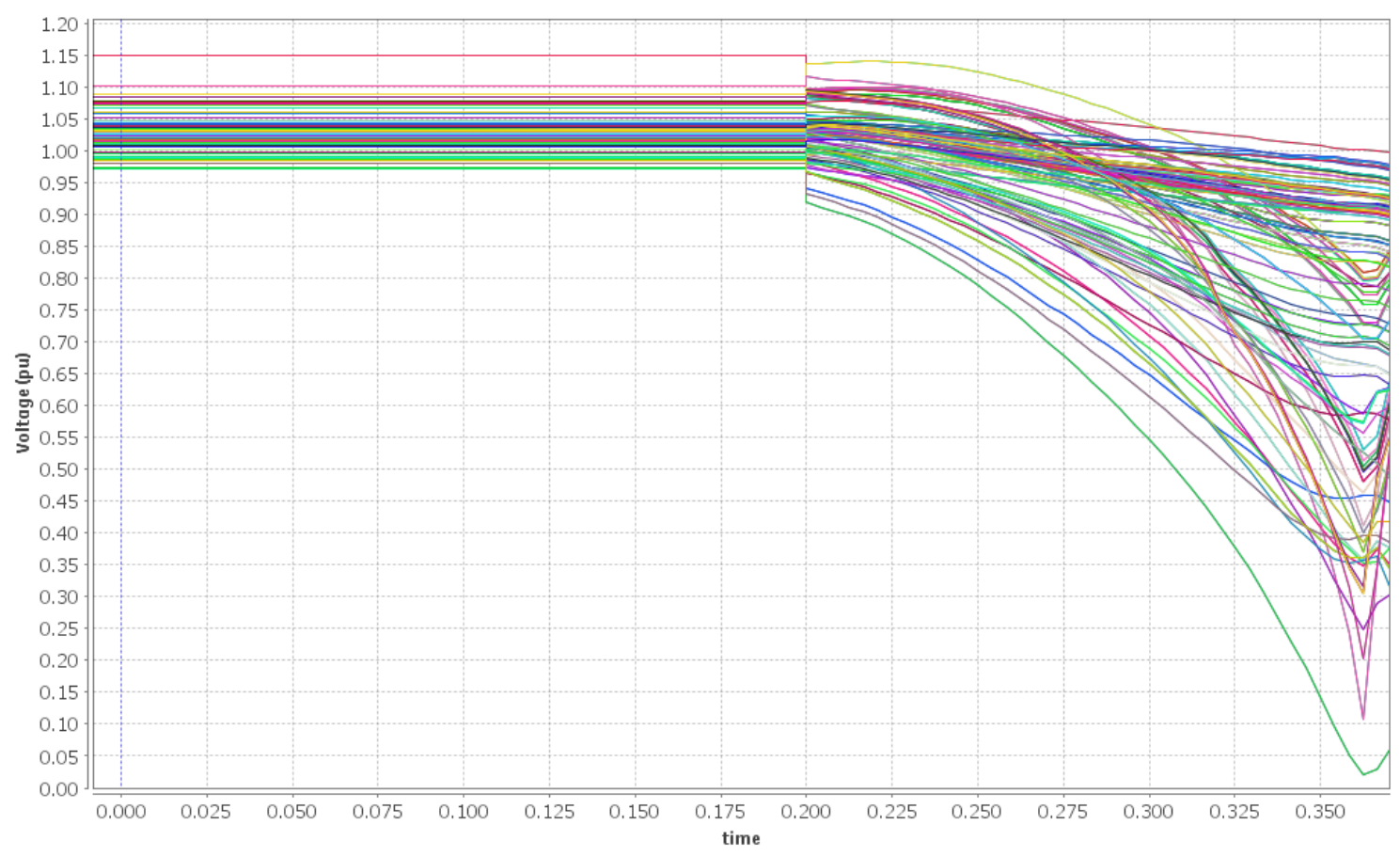

Figure 73. Voltage with HVDC already blocked (N-1), trip Gateway West (N-1-1)

\subsubsection{N-1-1 with Remedial Action Scheme}

Current practice in WECC allows a remedial action scheme (RAS) with transfer trip of generation on certain AC contingencies or combinations of contingencies. Transfer trip limits in Area 65 (PACE) were of particular interest in this case. Based on input from the technical review committee, this work set the maximum allowed amount of remedial tripping at $600 \mathrm{MW}$. This value was determined based on the loss of the largest unit in the area and ensured that the system could accommodate this level of generation trip without major unintended consequences.

With the HVDC link blocked and the subsequent loss of Gateway West, it was obvious that the system would have difficulty exporting power through the lower $\mathrm{kV}$ system. Therefore, the RAS scheme devised reduced $600 \mathrm{MW}$ of generation on the exporting end of the corridors, i.e., near the Aeolus area. The Snowy Range substation stood as a potential candidate for generation tripping, because the transmission reinforcement discussed above makes it a single large site a single bus away (with nearly 2,500 MVA of installed wind capacity).

Figure 74 show the response of all bus voltages rated $230 \mathrm{kV}$ and above in Area 65 (PACE) with the loss of Gateway West with the RAS scheme implemented. Gateway west was tripped at 0.2 $\mathrm{s}$, with $600 \mathrm{MW}$ of wind tripped after that, at $0.25 \mathrm{~s}$. The speed of all generating units in Area 65 (PACE) and Area 73 (WAPA RM) are shown in Figure 75 and Figure 76, and they indicate a system-wide loss of stability. Notice also that the high voltage observed at 67811 ANPOPC\&1 $500 \mathrm{kV}$ was corrected in this case. 


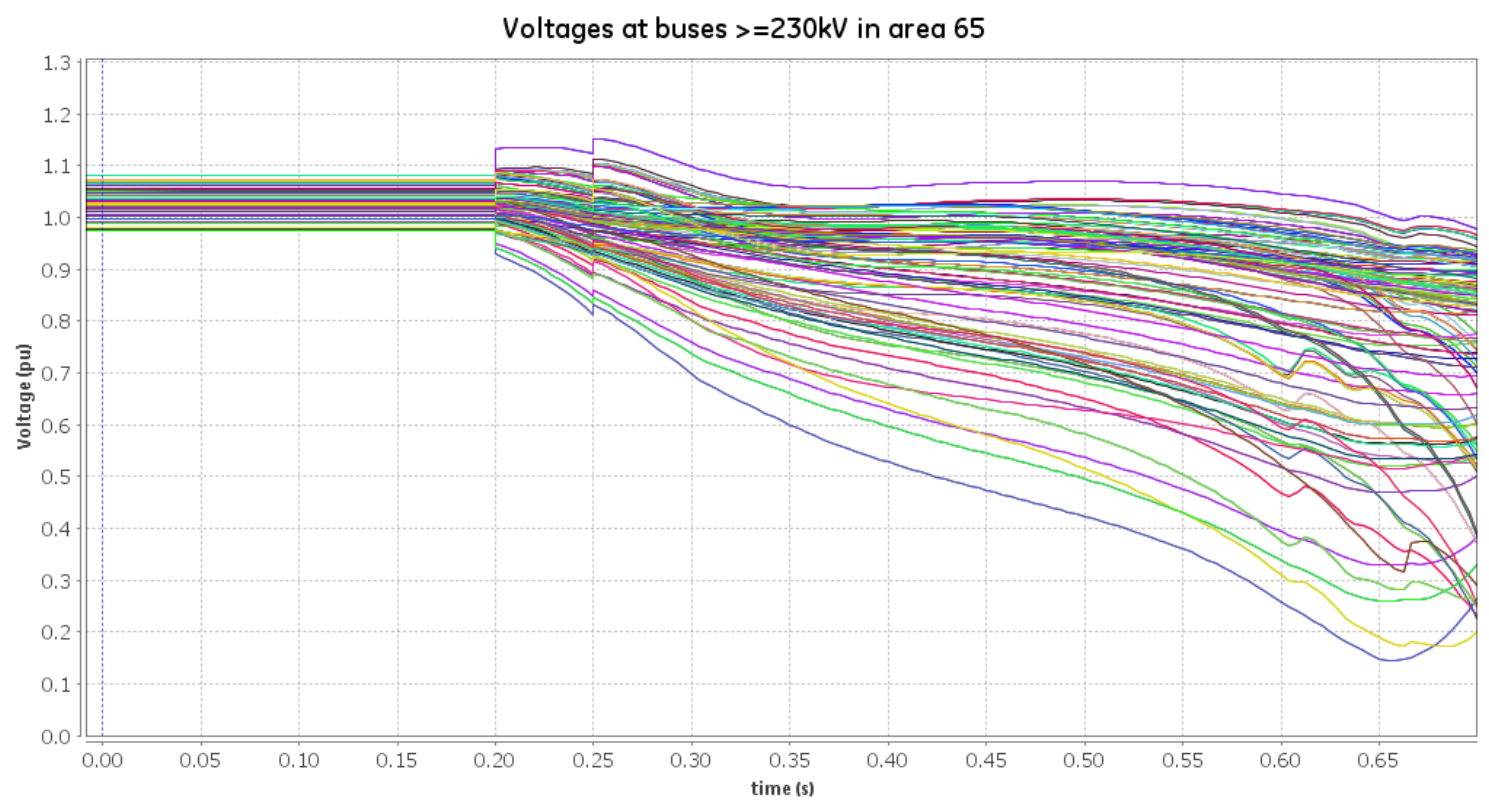

Figure 74. Voltage collapse with RAS trip of $600 \mathrm{MW}$ of wind at Snowy Range

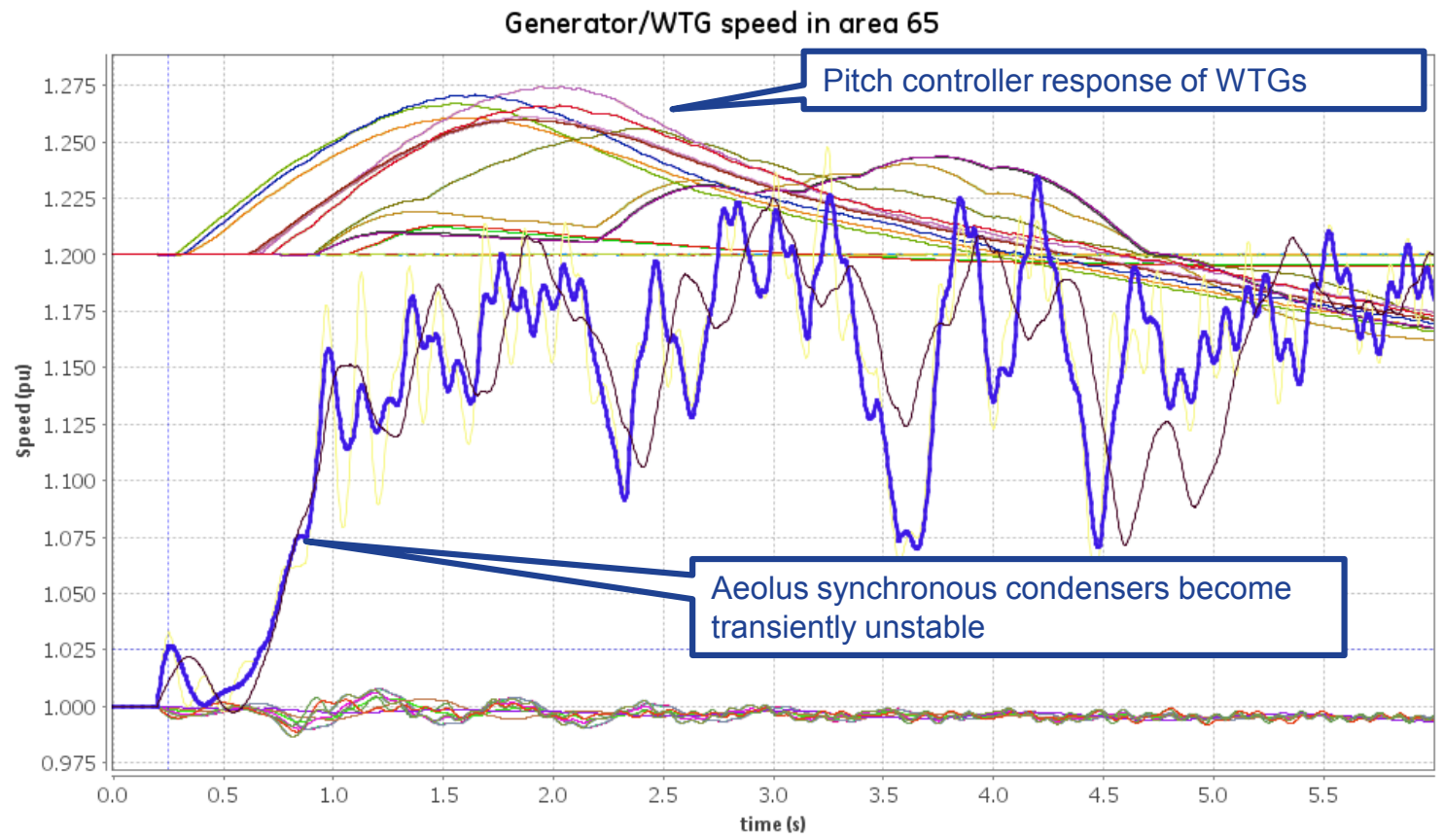

Figure 75. Speed of generation in Area 65 (PACE) 


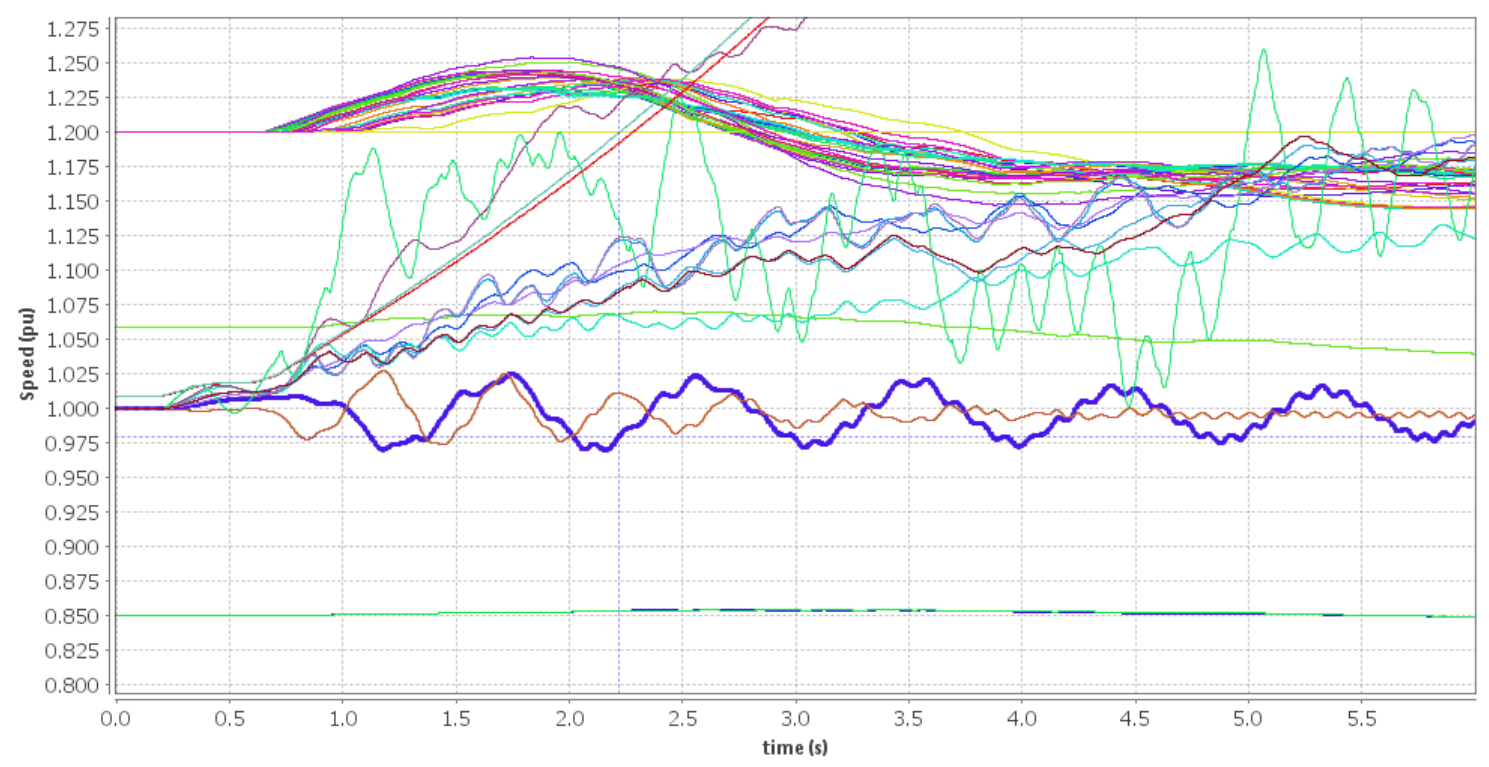

Figure 76. Speed of generation in Area 73 (WAPA RM)

To test whether more significant generation rejection would help the system attain stability, the level of generation trip was increased to higher levels. Additional simulations indicated that tripping approximately 1,400 MW of wind at Snowy Range $50 \mathrm{~ms}$ after the loss of Gateway West was enough to avoid the loss of system-wide stability. This suggests that improvements that would allow transfer tripping an additional $800 \mathrm{MW}(1,400 \mathrm{MW}-600 \mathrm{MW})$ of generation out of the area could avoid system-wide instability. Such action could be seen as a potential operator intervention in response to the loss of the HVDC link and must be taken before the Gateway West was also lost.

\subsubsection{System Repositioning After Loss of HVDC Link}

With the loss of the HVDC link, the exporting capability of the system was considerably reduced, and relied more on Gateway West for power transfers. Therefore, caution needed be exercised when the flows on Gateway West increased as a consequence of the HVDC blocking.

After blocking both HVDC poles and before tripping Gateway West, it was assumed that system operators would have some time to intervene and steer the system into a more secure state. These operator interventions take minutes to occur in practice. Among potential actions, one can cite generation dispatch, capacitor switching, power rerouting by PAR action, and others. However, additional unit commitment generally takes longer, so new generation commitment was not considered as a potential remedial action option for operators here. The choice of actions taken is normally defined based on the type of problem being experienced-e.g., voltage sags, frequency deviations. Based on the test of a large wind generation trip RAS, it appeared that preemptively reducing flows on the exporting end of Gateway West before it was tripped may have been sufficient to maintain system stability. To that end, a total of five wind power plants at buses $69073,69027,86267,86321$, and 86270 were given power orders of 0 MW and stopped generating power but continued to provide voltage support and regulation. In addition, two Type 1 wind units were tripped at Ft. Creek and disconnected because they intrinsically do not contribute to voltage support. 
This redispatch is a simple proxy for a real-time, security-constrained economic dispatch, with the plants curtailed to address post-contingency voltage and thermal problems and reposition the system into a safer state before another disturbance can take place. In this study, the curtailment was based on the proximity of the wind power plants to Gateway West instead of using an economic dispatch tool. As a consequence, governor action (rather than a new active power order from economic dispatch) shared the power imbalance among nearby units after the generators were curtailed.

Figure 77 shows the system response for the curtailment of approximately $600 \mathrm{MW}$ at the specified buses. System response was close to steady state $10 \mathrm{~s}$ after curtailment, and the simulation was run for an additional $60 \mathrm{~s}$. Figure 78 shows the remaining events applied to this scenario. At $\mathrm{t}=60 \mathrm{~s}$, Gateway West was tripped, and the RAS scheme tripping another $600 \mathrm{MW}$ of generation at Snowy Range was applied $50 \mathrm{~ms}$ after that, at $\mathrm{t}=60.05 \mathrm{~s}$. As observed in Figure 78 , system response was now stable but poorly damped. Voltages also settled at unacceptably low values, and some additional remediation was necessary.

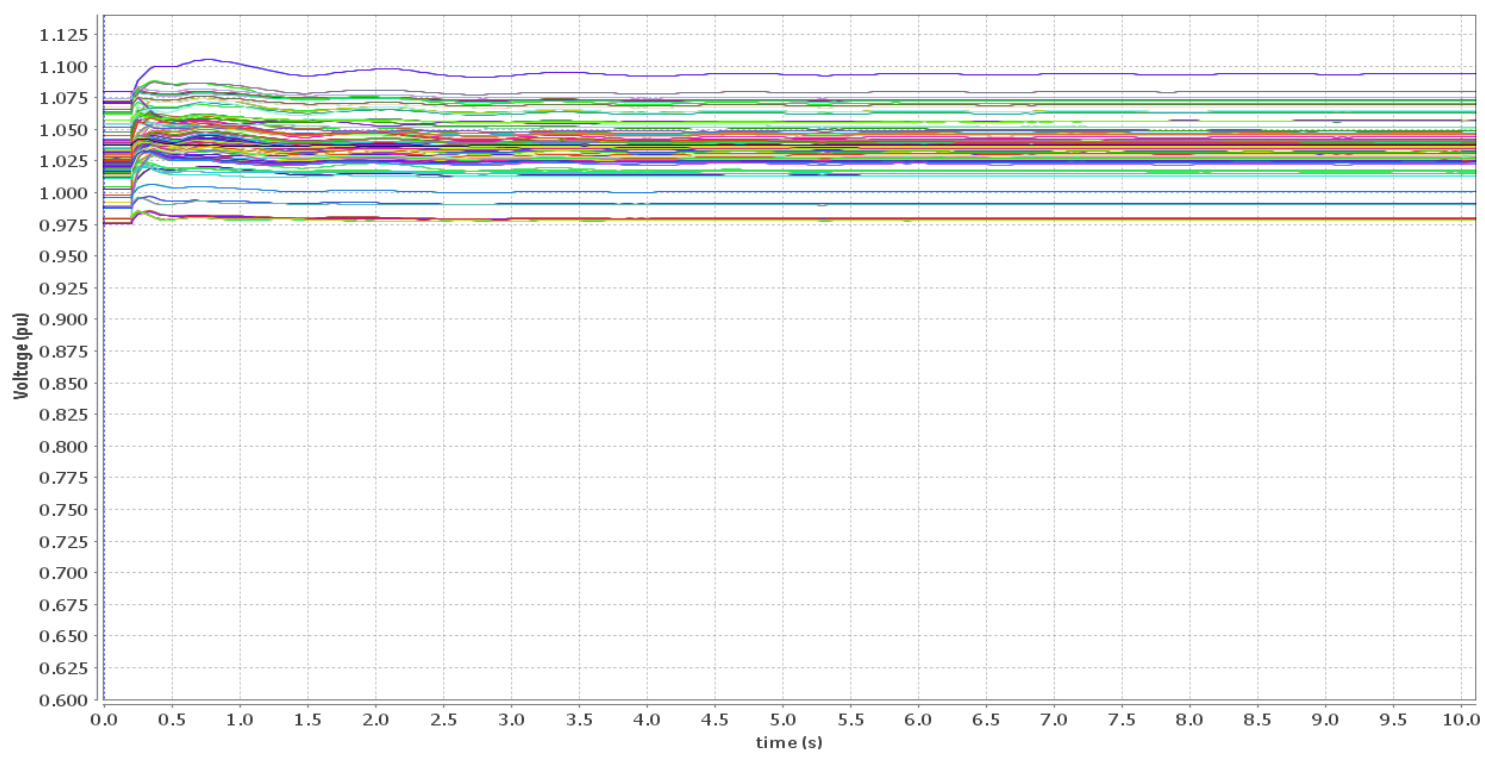

Figure 77. Trip of approximately $600 \mathrm{MW}$ at Snowy Range at $0.2 \mathrm{~s}$ 


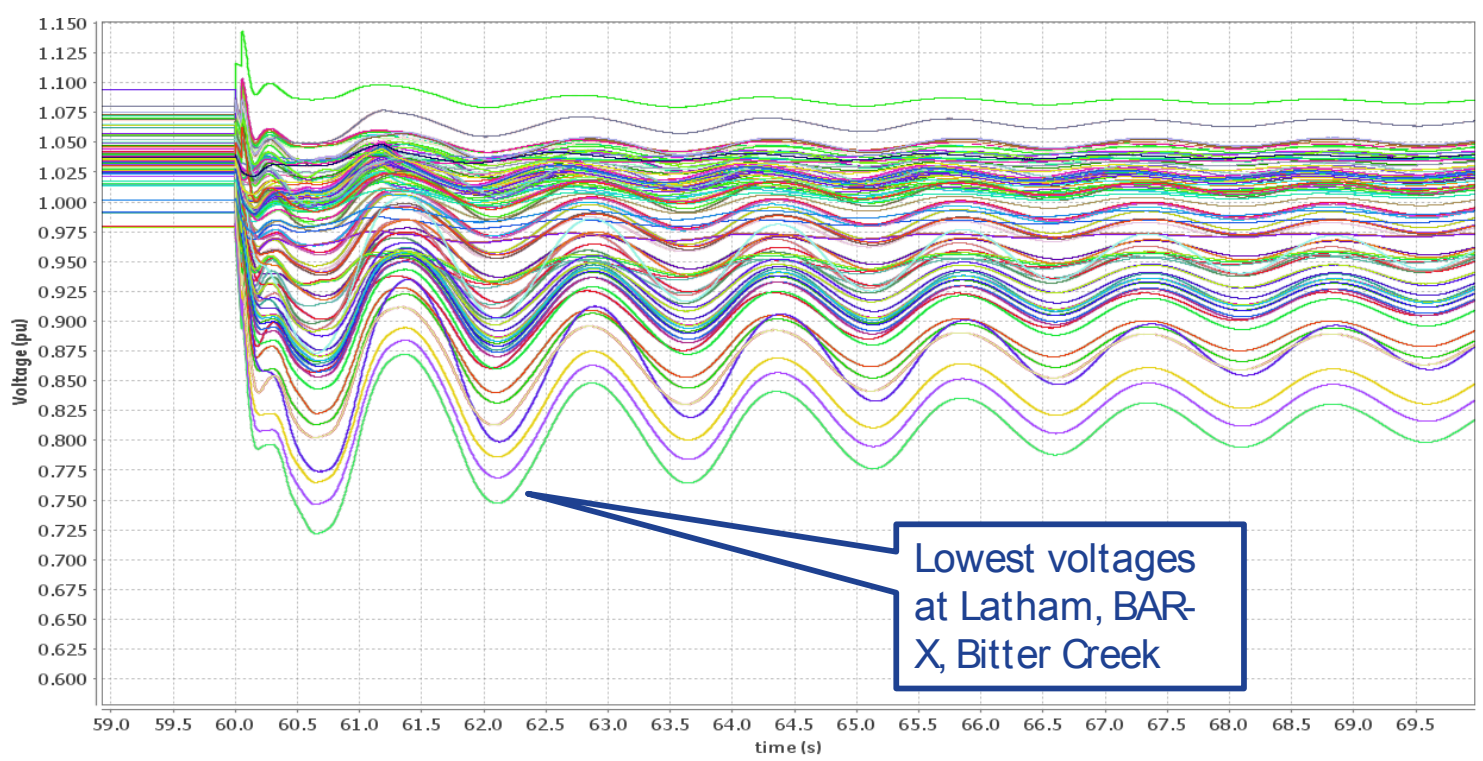

Figure 78. Gateway West trip with 600 MW RAS and repositioned system

\subsubsection{Further Conventional Reinforcements}

Conventional remediation of low post-contingency voltages included the addition of reactive power compensation devices. To that end, Bridger 1 unit was converted to a synchronous condenser to help improve voltages in the vicinity. Although reactive power generation at the Bridger synchronous condenser responded accordingly to regulate terminal voltage, it was not sufficient to improve voltages at the buses where the sags were more prominent.

After identifying the buses that had the lowest voltages, as shown in Figure 78 and on the map in Figure 79 , it was noticed that the low voltages occurred along the $230-\mathrm{kV}$ radial paths around Gateway West. These 230-kV circuits were now highly loaded given the fact that Gateway West was tripped and all power going west had to go through them now.

These localized low-voltage issues were addressed with 100-MVAr capacitor banks installed at Latham, Point of Rocks, Bar-X, and Mustang. The final steady-state condition is depicted in Figure 80, which shows considerable improvement in post-contingency voltages.

Figure 80 also indicated that the response was poorly damped. Although power system stabilizer tuning was not attempted, it could certainly be useful to improve damping and reduce settling time of oscillations. PSS tuning is outside the scope of this project. 


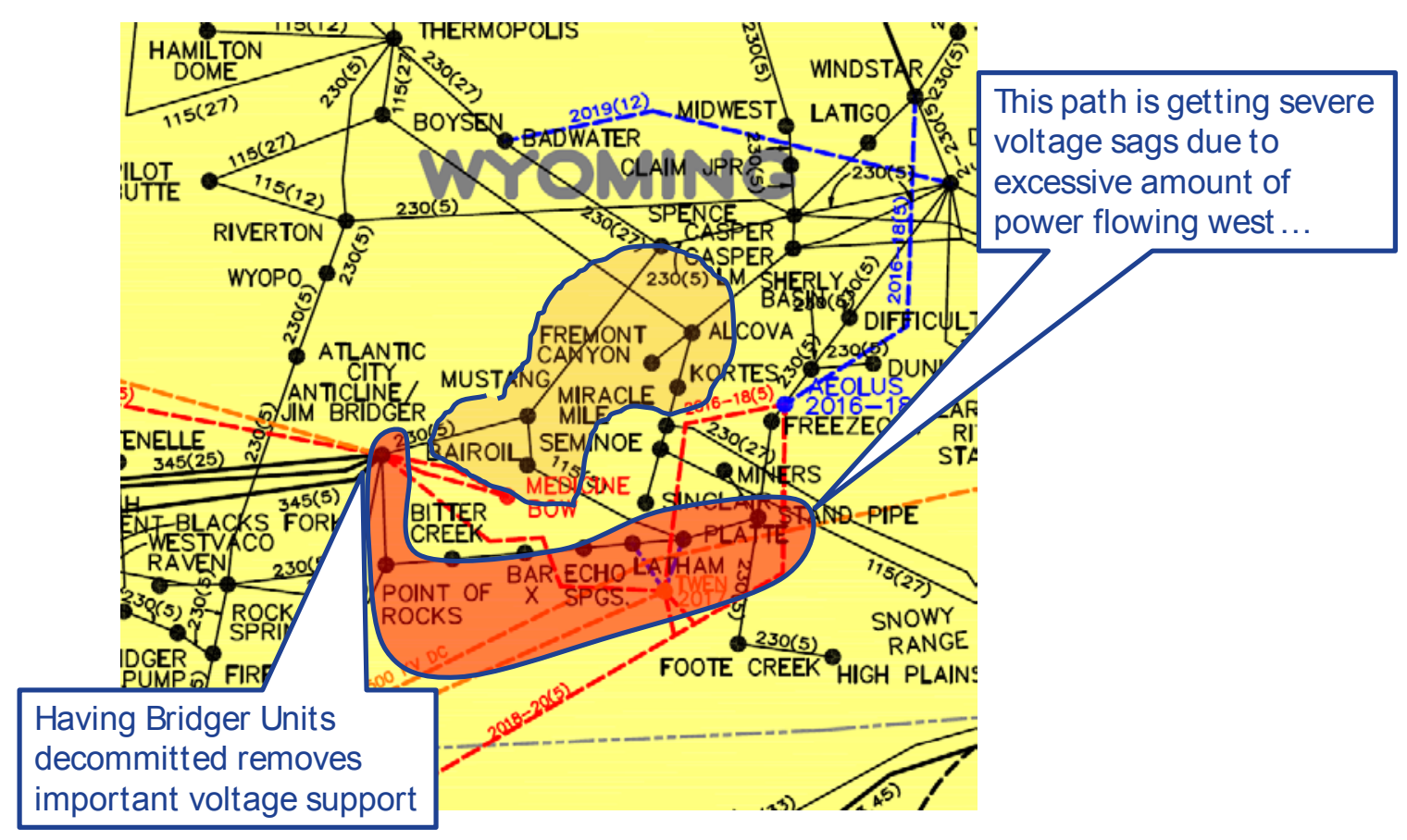

Figure 79. Regional 230-kV system stress

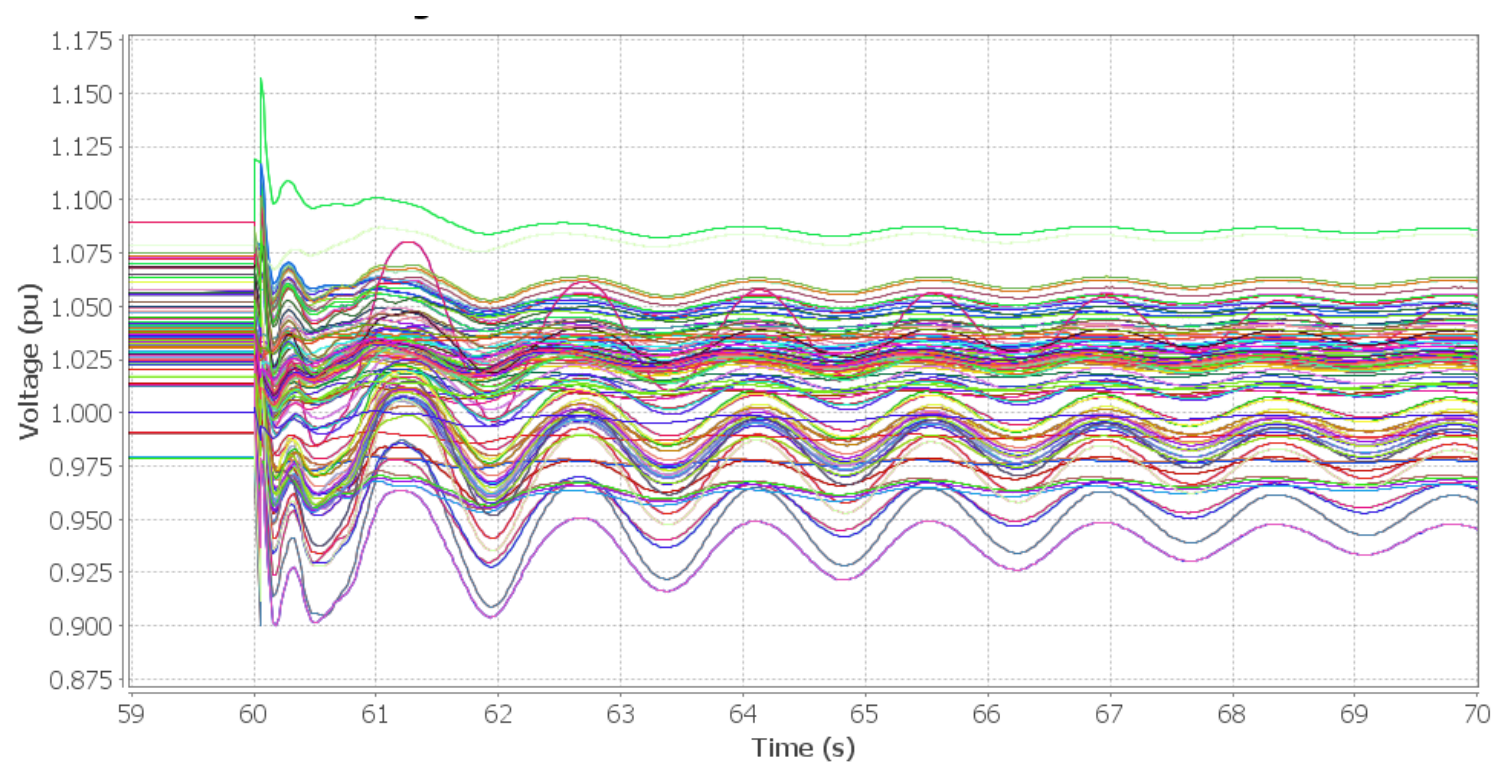

Figure 80. Voltages in Area 65 (PACE) with all reinforcements 


\subsection{HVDC Observations and Conclusions}

Based on the dispatch conditions and commitment tested, the incorporation of the HVDC link can help improve system robustness to faults and loss of transmission circuits. The 3,000-MW HVDC link relieved stress more than the 500-kV Gateway South AC project. The DC line carried more than 1,000 MW more than Gateway South, most of which reduced loading on Gateway West.

Because of this unloading of the Gateway West, the loss of the HVDC link carrying 3,000 MW of power was not critical as long as Gateway West was still in the case. It is important to emphasize that the system dispatch studied represented a light load condition because that is when the network had the least synchronous generation running. Gateway West was lightly loaded and could accommodate a large amount of power after the trip of the HVDC link. Additional investigations of heavily loaded scenarios, with Gateway West carrying higher flows, will help gain more insights on system-wide instability with the loss of the HVDC link.

The loss of Gateway West in addition to the loss of the HVDC system can be critical to systemwide instability. To avoid instability, the system needs to be reinforced to survive both contingencies. Tested reinforcements included the curtailment of $600 \mathrm{MW}$ of wind near Gateway West and an RAS to trip $600 \mathrm{MW}$ in Snowy Range. The conversion of one Bridger unit to a synchronous condenser and the addition of shunt capacitor banks to correct post-disturbance voltages were also used to eliminate localized low-voltage issues. This was an illustrative remediation plan and was not developed as part of an optimized reinforcement strategy.

Despite being stable, the N-1-1 system remained under significant stress. Additional investigation would need to be performed to evaluate highly loaded scenarios and contribute to better understanding of potential actions and their consequences. Different dispatches, including even higher wind power levels (or less thermal plant displacement), will add stress to the system and should be considered as part of diligent system planning. Overall, mitigation via redispatch, RAS, and dynamic and static reactive support has proven an effective solution in this exercise.

The issues observed in this study are not new to system planners and can be remediated with good planning practices. It is noteworthy to mention that when instability occurs on weak grids that have substantial amounts of renewable generation, the system becomes unstable faster than stronger grids do. This is a major outcome of this exercise, and it offers insights as to what needs to be covered in future planning practices.

\subsection{Non-Fundamental Frequency Stability Discussion}

Stability studies using fundamental frequency, positive-sequence tools will continue to be the main type of analysis for planning and operation, but not all phenomena of concern can be captured with these tools. Local aspects of interaction with large inverter systems, particularly HVDC systems, need to be evaluated with other tools that account for faster interactions that cannot be captured with current positive-sequence tools. Non-fundamental, frequency performance concerns are well known for HVDC applications, and they are always considered in detailed design studies. With the introduction of multiple $\mathrm{GW}$ of inverter-based generation in the electrical vicinity of the DC terminals, there is potential for additional interaction, and this must be considered. 
More broadly, with multiple GW of inverter-based generation in electrically dispersed systems, there is potential for additional interactions with other high-power converter equipment as well. For example, firing and automatic voltage regulator controls on a static VAR compensator have been observed to interact with fast current controls and phase-tracking systems in wind power plants. Further, there is potential for interactions among wind turbines and plants with equipment from different suppliers. These phenomena cannot be completely analyzed with fundamental frequency, positive sequence stability simulations.

In situations where proposed wind power plants are to be connected to weak systems, the WTG OEM should be appraised of the expected short-circuit strength at the point of interconnection and of large power electronic resources (including wind power plants, solar PV plants, HVDC, static VAR compensators, etc.) in the electrical proximity. The wind OEM may recommend that non-fundamental frequency analysis (such as point-on-wave time simulations or frequency domain analysis) be performed.

\subsection{Discussion of Good Engineering Practice}

This study, as well as WWSIS-3, show the need for "good engineering practice" to be followed. Although it is not possible to provide a complete tutorial on good practice, a short synopsis of key aspects of good practice is provided here.

- Thermal limits. Real and reactive power flows through all transmission lines and transformers should respect applicable thermal criteria under both normal operating and contingency conditions.

- Voltage stability and reactive compensation. Voltages should meet grid guidelines (e.g. WECC rules [WECC 2003]), and wind power plants should adhere to any grid code or interconnection requirements (e.g., closed-loop voltage regulation of the point of interconnection). Shunt and series capacitive compensation should be considered as means to improve system voltages, with due caution regarding voltage stability and transient overvoltage concerns. Voltage droop on plant regulators or other means may be needed to ensure coordination.

- Contingency performance (e.g., N-1). Evaluations should consider credible contingencies and adhere to grid planning guidelines. Contingencies that require grid reinforcements or specific control actions by wind power plants (e.g., fast curtailment) should be evaluated and designed during the planning process.

This discussion does not mean that every historical practice should be followed, nor that practice shouldn't evolve. For example, the selection of limiting conditions for which N-1 must result in satisfactory performance requires careful scrutiny. The use of economically realistic initial conditions is important. Limiting the addition of new wind generation (or other infrastructure) by requiring satisfactory thermal, voltage, and stability performance for conditions that are economically or physically unlikely is not good engineering practice. Planners should avoid evaluations of new plants that unrealistically assume no change in system operations (particularly the commitment and dispatch of existing generation). 


\section{Summary and Conclusions}

\subsection{Key Observations on Low Levels of Synchronous Generation}

The investigation of transient stability in the eastern portion of the Western Interconnection under conditions of low levels of synchronous generation produced the following insights:

- As with any significant system change, thermal and voltage issues must be addressed before stability issues. Standard techniques can be employed, such as new transmission, reactive compensation, adjusted commitment, and dispatch.

- Transient stability issues in exporting regions dominated by wind can look different. Behavior is more closely akin to traditional fast voltage collapse than machine-angle instability. Stability failures can occur very rapidly.

The failures observed are moderately localized, because the study area is both highly stressed and on the eastern edge of the Western Interconnection. A key finding is that the remediation of performance problems includes two broad classes of technology:

- Standard grid-based fixes, such as reduced loading, transmission reinforcements, remedial action schemes, and reactive compensation with adequate dynamic range

- The use of control features on wind turbines and wind power plants. Note:

$\bigcirc$ The use of features in the WECC second-generation, generic models was effective.

- Individual manufacturer controls can be highly effective.

- Wind generation behavior during disturbances varies among manufacturers and features. Attention should be given to the features and performance of specific wind power plants when they are evaluated during the generation interconnection process.

Grid-based solutions and wind power plant controls for weak grids are complementary. Wind power plants that have higher functionality tend to reduce the need for grid-based solutions and vice versa. Costs and benefits of the various means of mitigation (e.g., grid-based operational solutions, grid reinforcements, added control features on new wind power plants) should be analyzed to ensure a technically and economically viable solution.

Finally, an analysis of transient stability limitations requires careful attention to models and tools. For instance:

- Simulations with greatly reduced synchronous generation and high stress are strongly affected by details of wind power plant, load, and reactive compensation models.

- After plants are constructed, performance and modeling should be verified for addition to common study data sets.

- Standard transient stability tools using fundamental frequency, positive sequence have built-in approximations that can affect results, particularly when stability limits are hit in very short (e.g., a few cycles) time frames. Enhanced voltage stability analysis tools are 
needed. Point-on-wave and frequency domain analysis may be required in some circumstances to augment fundamental frequency, positive sequence analysis.

- Other inverter-based generation (e.g., PV) and resources (e.g., battery energy storage), which have many similarities in dynamic performance characteristics, can be expected to present similar grid behavior and modeling challenges.

\subsection{Observations on HVDC Transmission}

The high-level investigation reported here suggests that the integration of large blocks of HVDC for export is feasible. Substantial, project-specific engineering far beyond the scope of this investigation would be needed to pick DC technology, rate equipment, and design integration. This investigation provides some observations specific to HVDC for export from regions that have high levels of wind and low levels of synchronous generation:

- A large DC system will have the potential to disrupt the system if not properly integrated.

- Familiar challenges associated with HVDC blocking will need to be carefully considered. Fast voltage support and adapting to rapid counterflows on secondary AC transmission can be challenging. Dynamic reactive compensation is important.

- The conversion of a coal power plant to a synchronous condenser was found again to be beneficial.

- This investigation did not identify a compelling reason to favor LCC or VSC (nor was it intended to), and careful evaluation of specific DC technologies is necessary for each application.

- The potential for local, non-fundamental frequency interaction between inverter-based generation (wind and solar) and large HVDC of any technology must be considered with a predominantly wind-exporting system.

\subsection{Conclusions}

This investigation shows that the integration of large amounts of wind generation in an exporting region with the associated displacement of substantial thermal generation is technically feasible.

It is possible to have transient stability problems, particularly if the system is unduly stressed. The stability problems can look somewhat different. Faults that occur when the transmission system is overloaded in violation of normal operating rules can result in fast voltage collapse and separation.

For the conditions studied - which included highly stressed power flow conditions, high wind and solar generation levels, and the vast majority of major coal generation in the region offline - system stability can be maintained with available wind power plant controls and limited, standard technology grid support.

This is relatively new ground for the industry, and this investigation is not a substitute for detailed planning, but the risks illustrated can be analyzed and mitigated. Tools, data, and the current state-of-the-art interconnection and bulk power system stability studies, if used following good system engineering practice as the system is built out, will ensure continued reliability of the power system. 


\subsection{Future Work}

This work illuminates a number of areas for which further study, analysis using different tools, and the development of new tools would move the industry's understanding of grid operations with low levels of synchronous generation forward.

\subsubsection{Western Interconnection Analysis}

Further investigations of different network topologies with low levels of synchronous generation (e.g., Southern California) would be of particular interest. The concern here is likely to be postfault voltage recovery under peak load, weak grid conditions rather than the dynamic response of renewable generation.

\subsubsection{HVDC Options}

Investigations of the relative merits of dedicated compared to embedded HVDC transmission projects for the delivery of wind power to load centers should consider the economics of operations (i.e., include production simulations) and stability evaluation, with particular attention to identifying stability limits, such as the impact on relevant path ratings. The investigations could also continue the evaluations of the relative merits of different HVDC technologies (i.e., VSC compared to LCC and point-to-point compared to multi-terminal).

\subsubsection{Voltage Stability Tools}

There is a need to develop and adapt voltage stability analysis tools to help planners understand the risks of the types of instability shown in this study. Methods to quickly and meaningfully calculate PV and QV curves that reflect the dynamic constraints of a system that has a preponderance of inverter-based resources would be a valuable complement to traditional transient stability simulations. Similarly, and ideally in coordination, the development of new screening tools based on short-circuit strength and the size, amount, and character of nearby inverter-based generation would be valuable for both planners and developers.

\subsubsection{Model and Method Validation}

Analyses of system stability using fundamental frequency, positive sequence analysis are well established and include well-known approximations. This remains the case with high levels of wind and solar. However, the industry's understanding and quantification of the limits of, and the errors introduced by, these modeling assumptions when applied to weak grids that have high levels of inverter-based generation is less well established than for grids dominated by synchronous machines. The use of other tools - such as lab tests on actual equipment, simulations with point-on-wave tools, and field measurements to complement and further validate the models - would strengthen the industry's understanding.

\subsubsection{Protection and Relaying Investigation}

Analyses of system stability using fundamental frequency, positive sequence analysis, including those presented in this report, generally assume that protective relaying and protection (e.g., circuit breakers), will continue to perform as presently modeled. For example, the faults used in this study were assumed to be of a "standard" four-cycle duration. The fault clearing times used in stability studies are usually conservative approximations of actual protection system behavior. This behavior is based on the level and character of short-circuit currents, which are substantially different and less well known from inverter-based generation. Whether these assumptions are 
still valid in systems with predominantly inverter-based generation warrants further investigation. 


\section{References}

GE Energy. 2010a. Western Wind and Solar Integration Study. (Subcontract Report) NREL/SR550-47434. Golden, CO: NREL.

GE Energy. 2010b. Western Wind and Solar Integration Study: Executive Summary. (Subcontract Report) NREL/SR-550-47781. Golden, CO: NREL.

Khalil, H. 1996. Nonlinear Systems. Upper Saddle River, NJ: Prentice-Hall.

Lew, D., and G. Brinkman. 2013. Western Wind and Solar Integration Study Phase 2: Executive Summary. (Technical Report) NREL/TP-5500-58798. Golden, CO: NREL.

Lew, D., G. Brinkman, E. Ibanez, A. Florita, M. Heaney, B.-M. Hodge, M. Hummon, G. Stark, J. King, S.A. Lefton, N. Kumar, D. Agan, G. Jordan, and S. Venkataraman. 2013. Western Wind and Solar Integration Study Phase 2. (Technical Report) NREL/TP-5500-55588. Golden, CO: NREL.

Miller, N.W., M. Shao, S. Pajic, and R. D'Aquila. 2014a. Western Wind and Solar Integration Study Phase 3: Frequency Response and Transient Stability. (Subcontract Report) NREL/SR5D00-62906. Golden, CO: NREL.

Miller, N.W., M. Shao, S. Pajic, and R. D'Aquila. 2014b. Western Wind and Solar Integration Study Phase 3: Frequency Response and Transient Stability: Executive Summary. (Subcontract Report) NREL/SR-5D00-62906-ES. Golden, CO: NREL.

NERC. 2009. A Technical Reference Paper: Fault-Induced Delayed Voltage Recovery. Ver 1.2. (Technical Report) Washington, D.C.: June.

Tande, J., and G. Olav. 2000. "Exploitation of Wind-Energy Resources in Proximity to Weak Electric Grids. Applied Energy (65) April: 395-401.

Taylor, C.W. 1994. Power System Voltage Stability. New York: McGraw-Hill.

Vittal, V. 2003. Transient Stability and Control of Large Scale Power Systems. Power Systems Engineering Research Center Background Paper.

WECC. 2003. WECC Reliability Criteria. Salt Lake City, UT: April.

WECC. 2011. System Performance: TPL-001-WECC-CRT-2.1: Regional Criterion. Salt Lake City, UT. https://www.wecc.biz/Reliability/TPL-001-WECC-CRT-2.1.pdf.

WECC. 2012. Composite Load Model for Dynamic Simulations. Report 1.0. (Technical Report) Salt Lake City, UT: WECC Modeling and Validation Work Group. https://www.wecc.biz/Reliability/WECC\%20MVWG\%20Load\%20Model\%20Report\%2 0ver $\% 201 \% 200$.pdf. 